University of Tennessee Health Science Center UTHSC Digital Commons

\title{
Tetrahydroisoquinoline Neurotoxins in Parkinson Disease
}

Michael G. DeCuypere

University of Tennessee Health Science Center

Follow this and additional works at: https://dc.uthsc.edu/dissertations

Part of the Nervous System Diseases Commons, and the Neurosciences Commons

\section{Recommended Citation}

DeCuypere, Michael G. , "Tetrahydroisoquinoline Neurotoxins in Parkinson Disease" (2010). Theses and Dissertations (ETD). Paper 55. http://dx.doi.org/10.21007/etd.cghs.2010.0067.

This Dissertation is brought to you for free and open access by the College of Graduate Health Sciences at UTHSC Digital Commons. It has been accepted for inclusion in Theses and Dissertations (ETD) by an authorized administrator of UTHSC Digital Commons. For more information, please contact jwelch30@uthsc.edu. 


\title{
Tetrahydroisoquinoline Neurotoxins in Parkinson Disease
}

\begin{abstract}
The goal of this dissertation work was to (1) determine the distribution of several tetrahydroisoquinoline (TIQ) derivatives in rodent, normal human and Parkinson disease (PD) brain, (2) quantify the levels of these TIQ derivatives in common food sources in an effort to link specific food intake patterns with the development of PD and (3) examine the neurotoxicity of select TIQ derivatives in human dopaminergic cell culture. The TIQs are a family of monoamine alkaloids that share structural homology with 1-methyl-4-phenyl-1,2,3,6-tetrahyrdropyridine (MPTP), can be formed from dopamine or its oxidized metabolites and may be involved in the pathogenesis of monoaminergic cell death.
\end{abstract}

In our studies, we utilize enantiomeric-selective high-performance liquid chromatography with electrochemical detection (HPLC-EC) and liquid chromatography with tandem mass spectroscopy (LCMS/MS) to determine the concentrations of TIQ derivatives in brain, as well as in common dietary plants in the United States. The dopaminergic SH-SY5Y human neuroblastoma cell line was utilized to document the neurotoxicity of $\mathrm{N}$-methylated salsolinol derivatives.

Several TIQ derivatives were detected in all regions subjected to analysis. In general, salsolinols were present at higher concentrations than TIQ and its benzyl and methyl derivatives, especially in human brain. Moreover, salsolinols were concentrated in areas with increased dopamine synthesis and turnover such as the ventral midbrain and striatum, respectively. Significantly lower levels of $(R)$ salsolinol, $(S)$ salsolinol, $\mathrm{N}$-methyl-(R)salsolinol and $\mathrm{N}$-methyl-(S)salsolinol were found in the caudate nuclei of PD in comparison with normal human brain. $\mathrm{N}$-methyl-norsalsolinol was detected in all regions of rodent and human brain subjected to analysis. In comparison to normal human controls, $\mathrm{N}$-methyl-norsalsolinol levels were significantly lower in the substantia nigra and caudate nuclei from PD patients. $N$-methylnorsalsolinol-immunoreactivity co-localized with a general neuronal marker and a monoaminergic cell marker. $\mathrm{N}$-methyl-norsalsolinol-immunoreactivity was not observed in glia.

Our analysis of common foods revealed high concentrations of TIQ derivatives in banana, cherry, peach, grapefruit, avocado, button mushroom, leaf lettuce and celery. Enantiomeric salsolinol derivatives were detected as racemic mixtures, without evidence of stereoselective synthesis. Utilizing a lifetime food-item intake questionnaire, we identified trends for increased intake of several plants products in PD patients. Members of the rue (Rutaceae), banana (Musaceae), gourd (Cucurbitaceae), carrot (Apiaceae) and nightshade (Solanaceae) families were found to be highly consumed in both normal human and PD groups, and to concentrate several potentially neurotoxic TIQ derivatives.

We correlated indices of cellular energy production and cell viability in dopaminergic SH-SY5Y cells after exposure to $\mathrm{N}$-methyl-norsalsolinol and $\mathrm{N}$-methyl- $(R / S)$ salsolinol. Both toxins induce dose-dependent decreases in cell survival with $L_{50}$ values of $0.305 \mathrm{mM}$ and $0.377 \mathrm{mM}$ after $24 \mathrm{hrs}$, respectively. These results suggest that cell death induced by these $N$-methylated salsolinols is due to impairment of cellular energy supply, caused in particular by inhibition of mitochondrial complex I. Their concentration in dopaminergic regions of human brain and relatively potent cytotoxicity among TIQs makes these compounds strong candidates for both an endogenous and exogenous link to cell death in PD.

Document Type

Dissertation

Degree Name

Doctor of Philosophy (PhD) 


\section{Program}

Biomedical Sciences

Research Advisor

Mark S. LeDoux, M.D., Ph.D.

\section{Keywords}

HPLC-EC, LC-MS/MS, Neurodegeneration, Neurotoxins, Parkinson Disease, Tetrahydroisoquinolines

\section{Subject Categories}

Diseases | Medicine and Health Sciences | Nervous System Diseases | Neurosciences 


\author{
A Dissertation \\ Presented for \\ The Graduate Studies Council \\ The University of Tennessee \\ Health Science Center
}

In Partial Fulfillment

Of the Requirements for the Degree

Doctor of Philosophy

From The University of Tennessee

By

Michael G. DeCuypere

May 2010 
Chapter 2 (C) 2008 by Wiley-Liss, Inc.

Chapter 3 @ 2008 by International Society for Neurochemistry. All other material @ 2010 by Michael G. DeCuypere.

All rights reserved. 


\section{DEDICATION}

To my loving wife, best friend and eternal companion Norma.

With love and thanks for supporting me in all my endeavors into neuroscience and neurosurgery. You make my journey worthwhile. 


\section{ACKNOWLEDGEMENTS}

First and foremost, I would like to thank my research advisor and mentor, Dr. Mark LeDoux, for the training I received in his laboratory. His encouragement, advice and supervision in my research and medical endeavors are much appreciated. Of utmost importance to my career, I appreciate his time, effort and motivation in my education on scientific writing.

I would like to thank the members of my graduate committee, Drs. Angela Cantrell, Lawrence Reiter, Joseph Callaway and Duane Miller, for their valuable comments and advice on my qualifying exam, research proposal and dissertation formulation.

I would also like to sincerely thank all the current and former members in Dr. LeDoux's laboratory: Dr. Jianfeng Xiao, Dr. Suzhen Gong and Dr. Yu Zhao, for their technical assistance, suggestions and camaraderie. In particular, I would like to thank Dr. Yu Zhao for her extensive training and assistance in cell culture techniques and valuable input regarding my experiments. Her scientific knowledge and friendship were welcoming and valued in the often-times rough seas of graduate school.

During my graduate school training I have had the privilege to learn from several faculty members in the Department of Anatomy and Neurobiology. My sincere thanks to: Drs. Robert Foehring, William Armstrong and Robert Waters for their instruction in neuronal physiology; to Dr. Matt Ennis for instruction in neuroanatomy; to Drs. Melburn Park and Lawrence Reiter for instruction on techniques in microscopy; to Drs. Angela Cantrell and Thomas Schikorski for their instruction on critical review of literature. I am especially grateful to Dr. Pat Ryan, IPBS program director, and Dr. Joseph Callaway, neuroscience graduate program director, who provided me with direction and support from the very beginning of my foray in neuroscience.

Many thanks to the staff in the Departments of Neurology and Anatomy and Neurobiology, including Brenda Smith for her administrative help in graduate school and Mary Reed, Bobbie Scott, and Frances Grigsby for their help in reagent purchasing and administrative work.

I would especially thank my wife and my extended family for their incredible love and support throughout my graduate training.

Finally, I would like to thank the National Institute of Neurological Disorders and Stroke for financial support (R03-NS049123 to MSL). 


\begin{abstract}
The goal of this dissertation work was to (1) determine the distribution of several tetrahydroisoquinoline (TIQ) derivatives in rodent, normal human and Parkinson disease (PD) brain, (2) quantify the levels of these TIQ derivatives in common food sources in an effort to link specific food intake patterns with the development of PD and (3) examine the neurotoxicity of select TIQ derivatives in human dopaminergic cell culture. The TIQs are a family of monoamine alkaloids that share structural homology with 1-methyl-4phenyl-1,2,3,6-tetrahyrdropyridine (MPTP), can be formed from dopamine or its oxidized metabolites and may be involved in the pathogenesis of monoaminergic cell death.

In our studies, we utilize enantiomeric-selective high-performance liquid chromatography with electrochemical detection (HPLC-EC) and liquid chromatography with tandem mass spectroscopy (LC-MS/MS) to determine the concentrations of TIQ derivatives in brain, as well as in common dietary plants in the United States. The dopaminergic SH-SY5Y human neuroblastoma cell line was utilized to document the neurotoxicity of $\mathrm{N}$-methylated salsolinol derivatives.
\end{abstract}

Several TIQ derivatives were detected in all regions subjected to analysis. In general, salsolinols were present at higher concentrations than TIQ and its benzyl and methyl derivatives, especially in human brain. Moreover, salsolinols were concentrated in areas with increased dopamine synthesis and turnover such as the ventral midbrain and striatum, respectively. Significantly lower levels of $(R)$ salsolinol, $(S)$ salsolinol, $N$ methyl- $(R)$ salsolinol and $N$-methyl- $(S)$ salsolinol were found in the caudate nuclei of PD in comparison with normal human brain. $N$-methyl-norsalsolinol was detected in all regions of rodent and human brain subjected to analysis. In comparison to normal human controls, $\mathrm{N}$-methyl-norsalsolinol levels were significantly lower in the substantia nigra and caudate nuclei from PD patients. $N$-methyl-norsalsolinol-immunoreactivity colocalized with a general neuronal marker and a monoaminergic cell marker. $N$-methylnorsalsolinol-immunoreactivity was not observed in glia.

Our analysis of common foods revealed high concentrations of TIQ derivatives in banana, cherry, peach, grapefruit, avocado, button mushroom, leaf lettuce and celery. Enantiomeric salsolinol derivatives were detected as racemic mixtures, without evidence of stereoselective synthesis. Utilizing a lifetime food-item intake questionnaire, we identified trends for increased intake of several plants products in PD patients. Members of the rue (Rutaceae), banana (Musaceae), gourd (Cucurbitaceae), carrot (Apiaceae) and nightshade (Solanaceae) families were found to be highly consumed in both normal human and PD groups, and to concentrate several potentially neurotoxic TIQ derivatives.

We correlated indices of cellular energy production and cell viability in dopaminergic SH-SY5Y cells after exposure to $\mathrm{N}$-methyl-norsalsolinol and $\mathrm{N}$-methyl$(R / S)$ salsolinol. Both toxins induce dose-dependent decreases in cell survival with $\mathrm{LC}_{50}$ values of $0.305 \mathrm{mM}$ and $0.377 \mathrm{mM}$ after $24 \mathrm{hrs}$, respectively. These results suggest that cell death induced by these $N$-methylated salsolinols is due to impairment of cellular energy supply, caused in particular by inhibition of mitochondrial complex I. Their concentration in dopaminergic regions of human brain and relatively potent cytotoxicity among TIQs makes these compounds strong candidates for both an endogenous and exogenous link to cell death in PD. 


\section{TABLE OF CONTENTS}

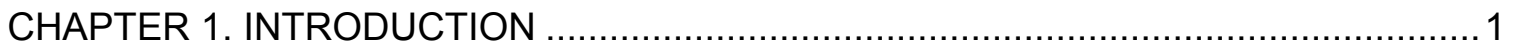

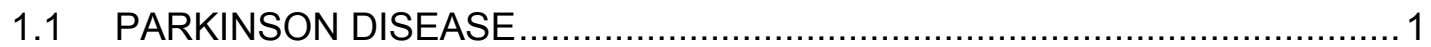

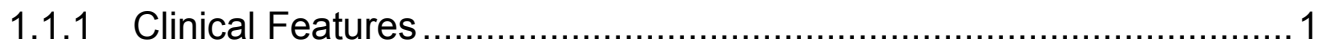

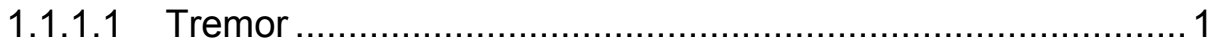

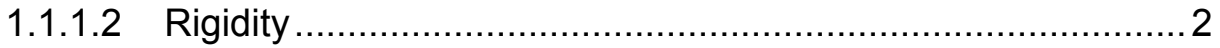

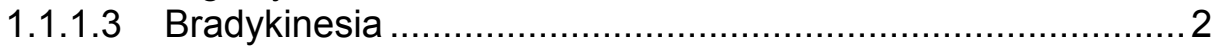

1.1.1.4 Parkinsonian Gait ......................................................

1.1.1.5 Secondary Manifestations ………................................... 3

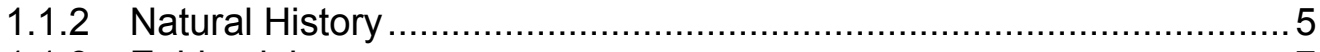

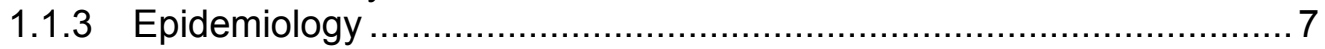

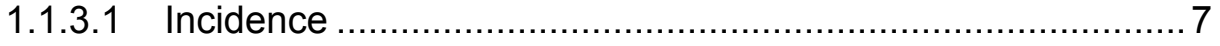

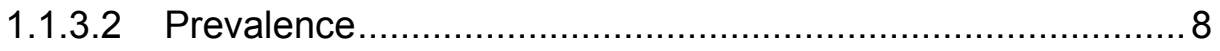

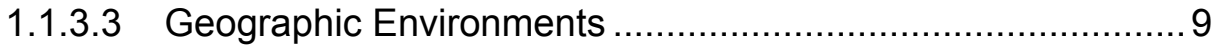

1.1.3.4 Genetic Risk Factors ……........................................ 9

1.1.4 Cellular and Molecular Pathology ……………........................... 10

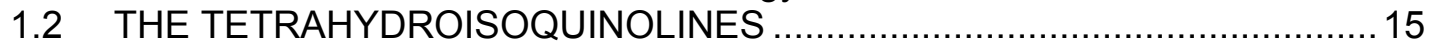

1.2.1 Neuronal Production ........................................................... 17

1.2.2 Environmental Sources.......................................................... 19

1.2.3 TIQs and the Blood-Brain Barrier ..............................................20

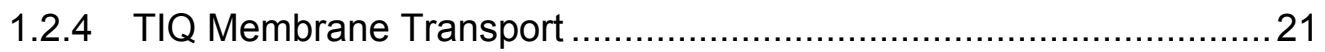

1.3 TOXICITY OF TETRAHYDROISOQUINOLINES .................................21

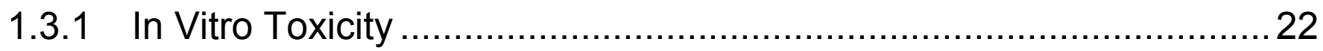

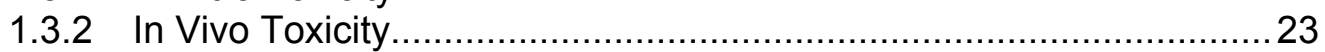

1.4 TETRAHYDROISOQUINOLINES IN PARKINSON DISEASE .....................24

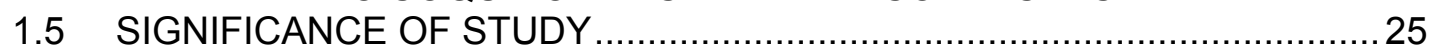

CHAPTER 2. LOCALIZATION OF N-METHYL-NORSALSOLINOL WITHIN RODENT

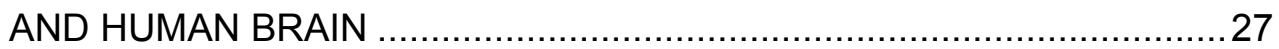

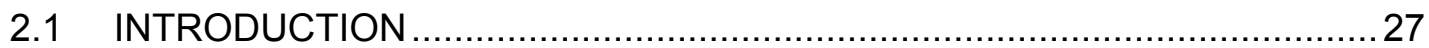

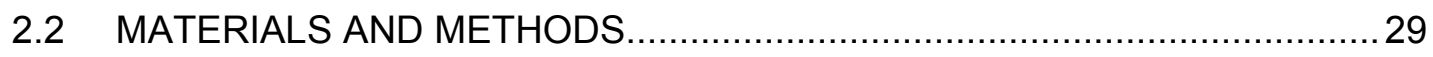

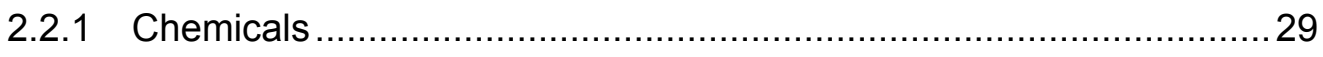

2.2.2 Synthesis of Derivatives of TIQ and Isoquinoline ...........................29

2.2.3 HPLC-EC Analysis of N-Methyl-Norsalsolinol ................................. 30

2.2.3.1 Chromatography Standard and Sample Preparation.............30

2.2.3.2 Mobile Phase Preparation ………………..........................31

2.2.3.3 Liquid Chromatography .................................................. 31

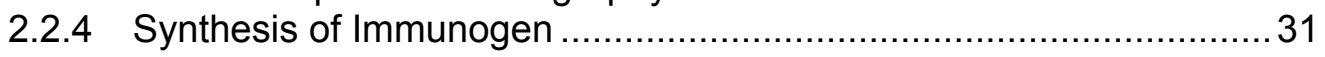

2.2.5 Production of Monoclonal Antibody ………................................... 31

2.2.6 Antibody Characterization....................................................... 32

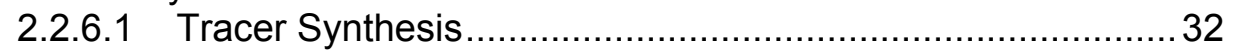

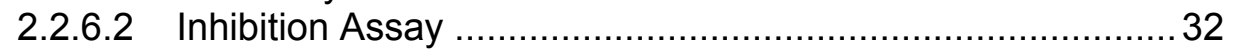

2.2.6.3 Absorption Control ............................................................ 32

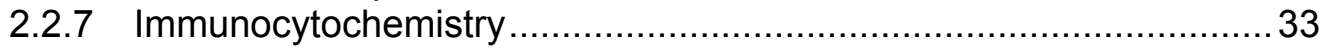

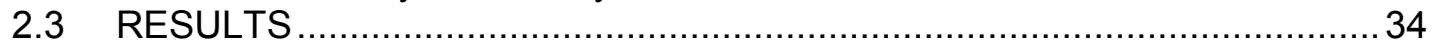

2.3.1 Quantitative Analysis of $\mathrm{N}$-Methyl-Norsalsolinol in Brain ....................34

2.3.2 Monoclonal Antibody to N-Methyl-Norsalsolinol ................................34

2.3.3 Localization and Distribution of $N$-Methyl-Norsalsolinol Immunoreactivity 


\subsubsection{Effect of Parkinson Disease on N-Methyl-Norsalsolinol}

Immunoreactivity........................................................................ 38

2.4 DISCUSSION

2.5 CONCLUSIONS

\section{CHAPTER 3. REGIONAL DISTRIBUTION OF TETRAHYDROISOQUINOLINE DERIVATIVES IN RODENT, HUMAN, AND PARKINSON DISEASE}

BRAIN

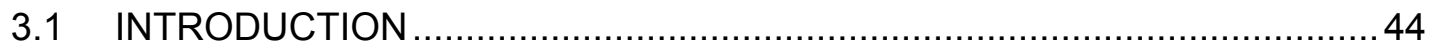

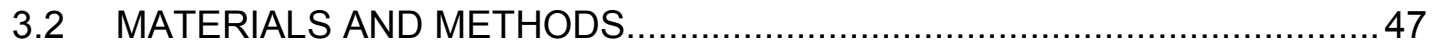

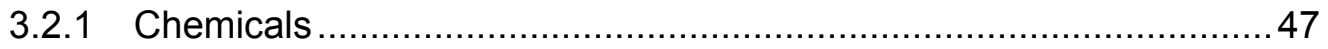

3.2.2 Synthesis of $\mathrm{N}$-methyl-(R/S)salsolinol .........................................48

3.2.3 Sample Preparation ........................................................... 48

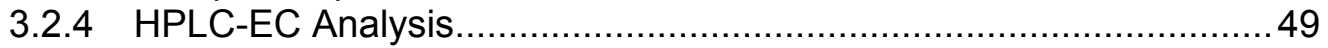

3.2.4.1 Chromatography Standard Preparation.............................. 49

3.2.4.2 Mobile Phase Preparation ..............................................49

3.2.4.3 Liquid Chromatography ............................................. 49

3.2.5 LC-MS/MS Analysis ............................................................. 51

3.2.5.1 Chromatography Standard Preparation...............................51

3.2.5.2 Liquid Chromatography .................................................51

3.2.5.3 Ion Trap Tandem Mass Spectrometry ………….................51

3.2.6 Compound Stability Experiments...............................................52

3.2.7 Statistical Analysis .................................................................. 52

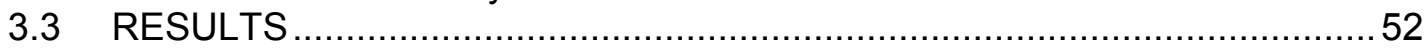

3.3.1 Quantitative Analysis of TIQ Derivatives in Brain ..............................52

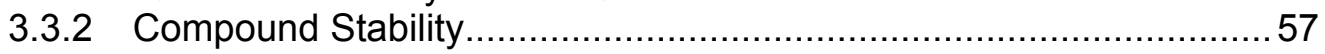

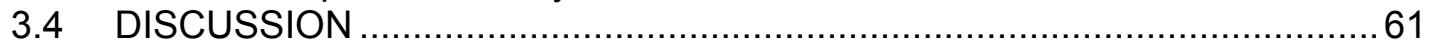

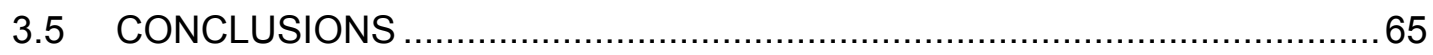

CHAPTER 4. ANALYSIS OF TETRAHYDROISOQUINOLINE DERIVATIVES IN COMMON FRUITS AND VEGETABLES AND THEIR

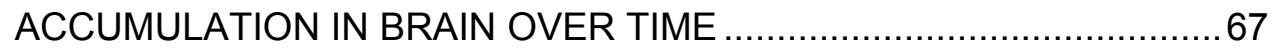

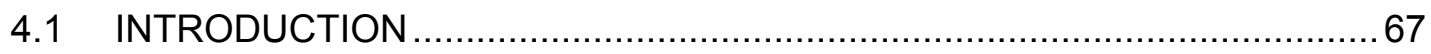

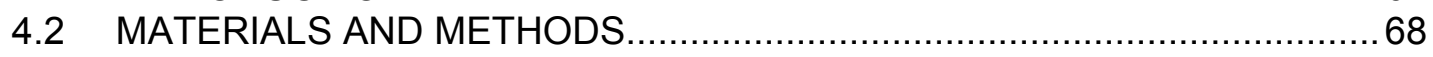

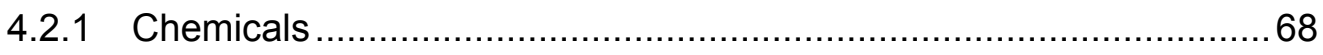

4.2.2 Synthesis of $N$-methyl-norsalsolinol and $N$-methyl- $(R / S)$ salsolinol ....68

4.2.3 Procurement and Preparation of Fresh Fruits and Vegetables ..........68

4.2.4 Rodent CNS Sample Preparation.................................................. 70

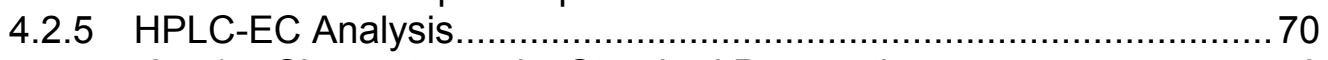

4.2.5.1 Chromatography Standard Preparation ................................70

4.2.5.2 Mobile Phase Preparation ……………………............... 71

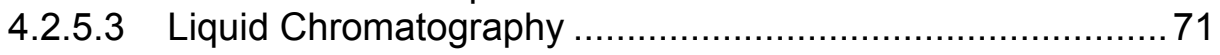

4.2.6 LC-MS/MS Analysis ...................................................................

4.2.6.1 Chromatography Standard Preparation ...............................71

4.2.6.2 Liquid Chromatography ……............................................ 72

4.2.6.3 Ion Trap Tandem Mass Spectrometry …….........................72

4.2.7 Parkinson Disease Lifetime Food Intake Questionnaire..................... 72

4.2.7.1 Instrument Development.............................................. 72

4.2.7.2 Instrument Statistical Analysis ........................................... 73

4.2.7.3 Reproducibility and Validity ............................................ 


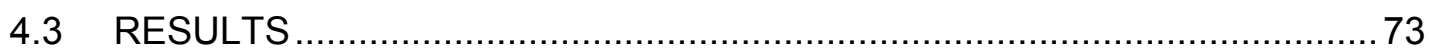

4.3.1 TIQ Derivatives in Common Fruits and Vegetables..........................73

4.3.2 Lifetime Intake of Fresh Fruits and Vegetables in PD Patients .........80

4.3.3 Reproducibility and Validity of the Survey Instrument ........................85

4.3.4 Accumulation of TIQ Derivatives in Rat Brain Over Time ..................85

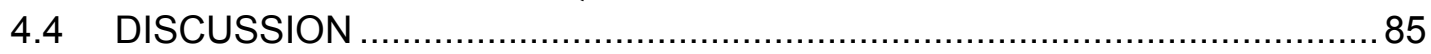

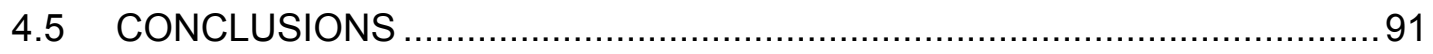

\section{CHAPTER 5. IN VITRO TOXICITY OF METHYLATED}

TETRAHYDROSIOQUINOLINE DERIVATIVES ......................................

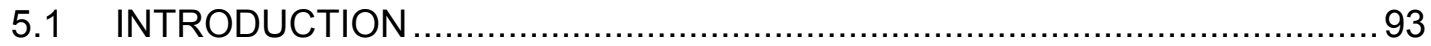

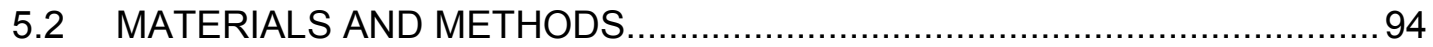

5.2.1 Chemicals ....................................................................... 94

5.2.2 Synthesis of $N$-methyl-norsalsolinol and $N$-methyl- $(R / S)$ salsolinol ....94

5.2.3 SH-SY5Y Cell Culture.................................................................. 94

5.2.4 Compound Handling and Dilution ................................................. 95

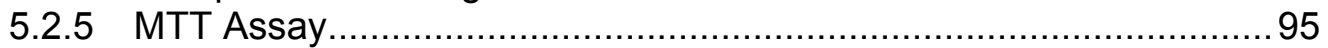

5.2.6 Measurement of Mitochondrial Membrane Potential $\left(\Delta \Psi_{m}\right) \ldots \ldots \ldots \ldots . . . .95$

5.2.7 DAPI Histochemistry ............................................................... 96

5.2.8 Calculation of Molecular Properties ............................................... 96

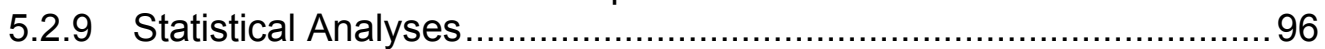

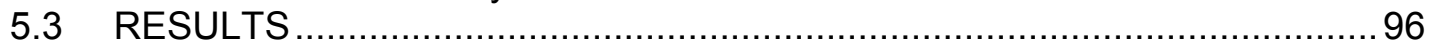

5.3.1 Cytotoxicity of TIQ Derivatives..................................................... 96

5.3.2 Decreased $\Delta \Psi_{\mathrm{m}}$ after Acute Exposure to Methylated TIQ

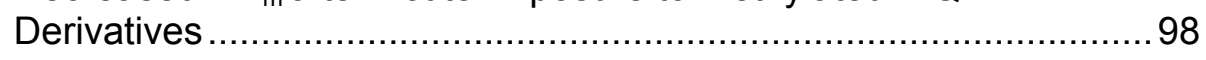

5.3.3 Nuclear Morphology after Exposure to TIQ Derivatives .................... 98

5.3.4 Molecular Properties of TIQ Derivatives ......................................... 98

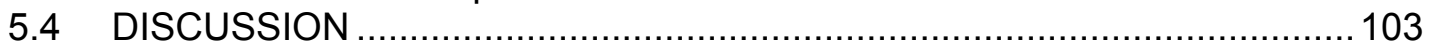

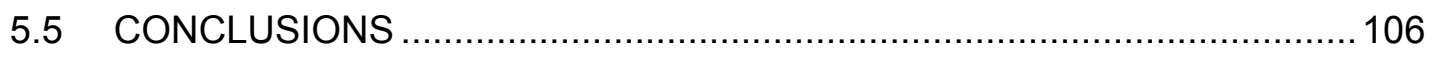

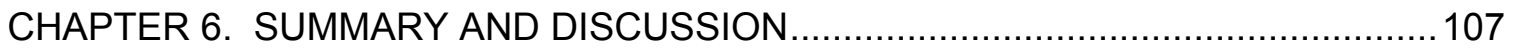

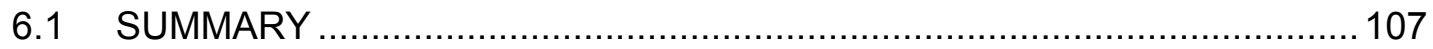

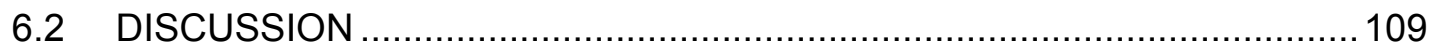

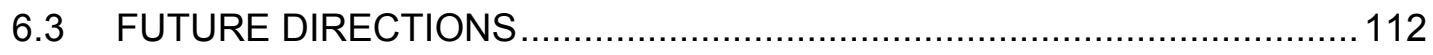

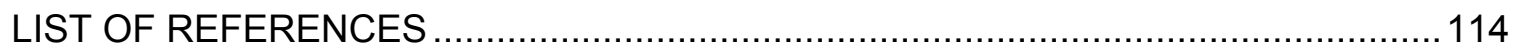

APPENDIX A. THERMAL STABILITY AND MOLECULAR PROPERTIES OF TETRAHYDROISOQUINOLINES ……………………..................146

APPENDIX B. PARKINSON DISEASE LIFETIME FRUIT AND VEGETABLE INTAKE QUESTIONNAIRE 


\section{LIST OF TABLES}

Table 2-1. Concentration of $N$-methyl-norsalsolinol within regions of rodent CNS ...... 35

Table 2-2. Concentration of $N$-methyl-norsalsolinol within regions of human CNS ....35

Table 2-3. Percentage (\%) cross-reactivities of the monoclonal antibody with compounds structurally related to $\mathrm{N}$-methyl-norsalsolinol

Table 3-1. Regional distribution of TIQ derivatives in rodent brain ..........................53

Table 3-2. Regional distribution of TIQ derivatives in normal human and PD brain ...55

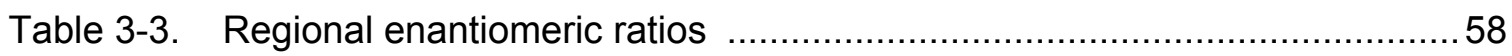

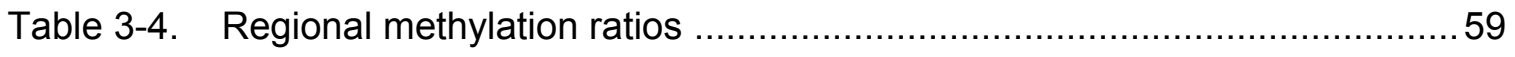

Table 3-5. Regional TIQ derivative/dopamine ratios in human brain .......................60

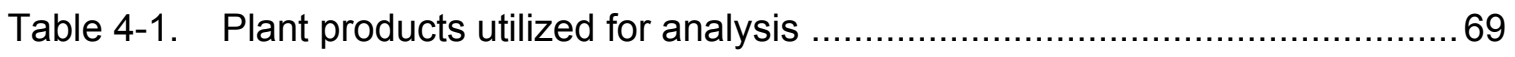

Table 4-2. Concentrations of TIQ derivatives in common fruits .............................74

Table 4-3. Concentrations of TIQ derivatives in common vegetables ......................76

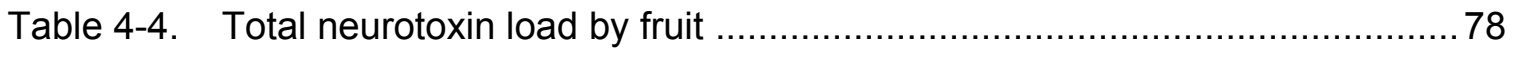

Table 4-5. Total neurotoxin load by vegetable ............................................. 79

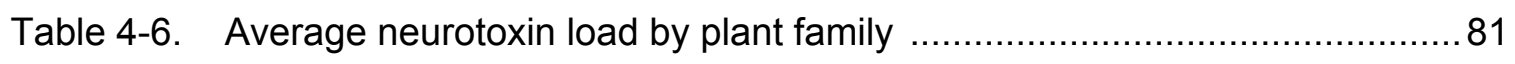

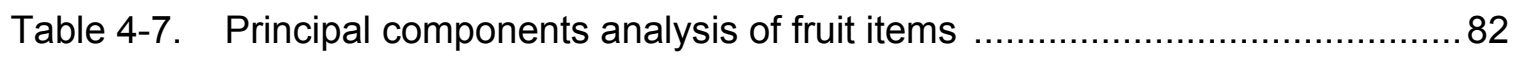

Table 4-8. Principal components analysis of vegetable items .............................. 83

Table 4-9. 95\% confidence intervals for principal components (mean lifetime

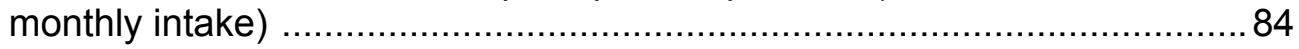

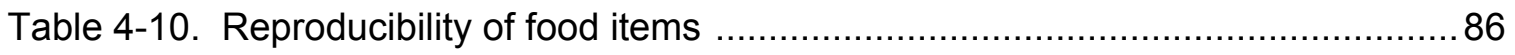

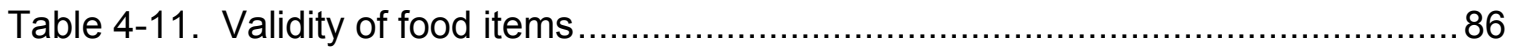

Table 5-1. Cytotoxicity and physical properties of study compounds .......................99

Table A-1. Limits of detection and quantification ......................................... 147

Table A-2. Molecular properties of TIQ derivatives and related compounds ............ 156 


\section{LIST OF FIGURES}

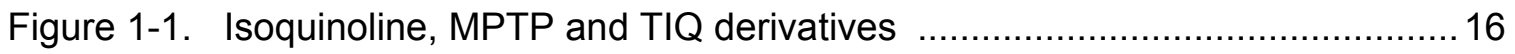

Figure 1-2. Overview of endogenous synthetic pathways .................................. 18

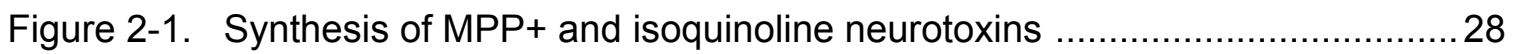

Figure 2-2. Cross-reactivities of the monoclonal antibody with compounds

structurally related to $\mathrm{N}$-methyl-norsalsolinol

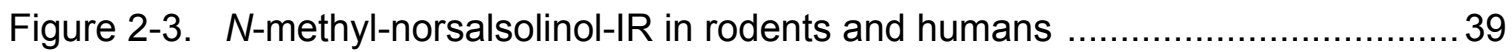

Figure 2-4. $\quad \mathrm{N}$-methyl-norsalsolinol-IR in the Substantia Nigra ............................... 40

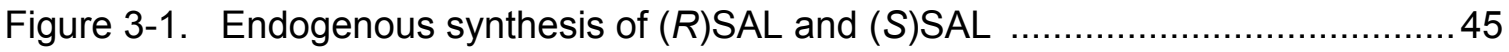

Figure 3-2. Chemical structures TIQ and its methylated $(A)$ and benzylated $(B)$ derivatives

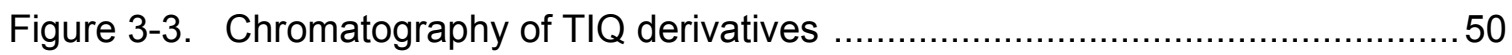

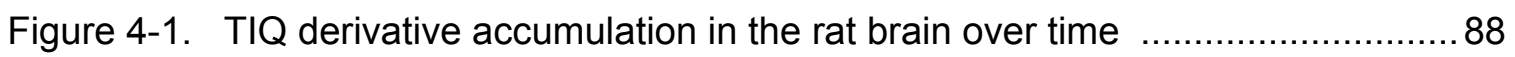

Figure 5-1. Viability of SH-SY5Y cells after 24 hour exposure to rotenone and TIQ derivatives

Figure 5-2. Viability of SH-SY5Y cells after 48 hour exposure to rotenone and TIQ derivatives

Figure 5-3. Decreased mitochondrial membrane potential $\left(\Delta \Psi_{\mathrm{m}}\right)$ after acute exposure to TIQ derivatives

Figure 5-4. Red/Green fluorescence ratios of study compounds over 1 hour 101

Figure 5-5. TIQ derivative-induced apoptosis of SH-SY5Y cells 102

Figure A-1. ${ }^{1} \mathrm{H}$ NMR spectra of $\mathrm{TIQ}$ 148

Figure A-2. ${ }^{1} \mathrm{H}$ NMR spectra of $N$-methyl-TIQ 149

Figure A-3. ${ }^{1} \mathrm{H}$ NMR spectra of 1-methyl-TIQ 150

Figure A-4. $\quad{ }^{1} \mathrm{H}$ NMR spectra of 1-benzyl-TIQ 151

Figure A-5. ${ }^{1} \mathrm{H}$ NMR spectra of $(R / S)$ salsolinol 152

Figure A-6. $\quad{ }^{1} \mathrm{H}$ NMR spectra of norsalsolinol 153

Figure A-7. $\quad{ }^{1} \mathrm{H}$ NMR spectra of $N$-methyl-(R/S) salsolinol 154 
Figure A-8. ${ }^{1} \mathrm{H}$ NMR spectra of $\mathrm{N}$-methyl-norsalsolinol 155 


\section{LIST OF ABBREVIATIONS}

\begin{tabular}{|c|c|}
\hline $\mathrm{AD}$ & Alzheimer disease \\
\hline CNS & central nervous system \\
\hline CSF & cerebrospinal fluid \\
\hline DA & dopamine \\
\hline DAT & dopamine transporter \\
\hline DOPAC & 3,4-dihydroxyphenylacetic acid \\
\hline DOPAL & 3,4-dihydroxyphenylacetaldehyde \\
\hline ETC & electron transport chain \\
\hline GBA & glucocerebrosidase \\
\hline GC-MS & gas chromatography with mass spectrometry detection \\
\hline Gl & gastrointestinal \\
\hline GPi & internal segment of globus pallidus \\
\hline HEK & human embryonic kidney \\
\hline HPLC-EC & high-performance liquid chromatography with electrochemical detection \\
\hline HVA & homovanillic acid \\
\hline IR & immunoreactivity \\
\hline $\mathrm{KO}$ & knockout \\
\hline LB & Lewy body \\
\hline LC-MS/MS & liquid chromatography with tandem mass spectrometry detection \\
\hline MAO & monoamine oxidase \\
\hline MPP+ & 1-methyl-4-phenylpyridinium ion \\
\hline MPDP & 1-methyl-4-phenyl-2, 3-dihydropyridium \\
\hline MPTP & 1-methyl-4-phenyl-1,2,3,6-tetrahydropyridine \\
\hline NAC & non-A $\beta$ component \\
\hline NACP & non- $A \beta$ component precursor \\
\hline NET & norepinephrine transporter \\
\hline NFT & neurofibrillary tangles \\
\hline NMR & nuclear magnetic resonance \\
\hline NNMT & nicotinamide $N$-methyl transferase \\
\hline PD & Parkinson disease \\
\hline PIGD & postural instability and gait disorder \\
\hline PS & Parkinsonian syndrome \\
\hline PSP & progressive supranuclear palsy \\
\hline SAL & salsolinol \\
\hline SNpc & substantia nigra pars compacta \\
\hline $\mathrm{TH}$ & tyrosine hydroxylase \\
\hline TIQ & 1,2,3,4-tetrahydroisoquinoline \\
\hline UPS & ubiquitin-proteasome system \\
\hline VIM & ventral intermediate nucleus \\
\hline WT & wild type \\
\hline
\end{tabular}




\section{CHAPTER 1. INTRODUCTION}

\section{$1.1 \quad$ PARKINSON DISEASE}

In the famous 1817 monograph "An Essay on the Shaking Palsy", James Parkinson first described the clinical condition that now bears his name (Parkinson 2002). His description was based on an account of six subjects, only three of whom he actually examined. The remaining three individuals were observed in public.

Remarkably accurate in his clinical descriptions, he single-handedly brought to life all of the cardinal features of the disease. Considering that few of his successors had improved upon this initial description, in 1872 Jean Martin Charcot dubbed the illness Parkinson disease (PD) (Charcot 1872-1873). Today, after almost 200 years of progress in understanding the pathophysiology of idiopathic PD, it remains a purely clinical diagnosis. In the current era of rapidly advancing medical technology, it is a humbling experience to the modern clinician that no ancillary test can prove the diagnosis of PD beyond the acuity of the neurologist's trained eye.

By definition, the presence of two out of three cardinal motor signs of parkinsonism (tremor, rigidity and bradykinesia) together with a consistent response to an adequate dose of levodopa are considered by most experts in the field to be essential for the diagnosis of PD to be made (Gibb \& Lees 1988). Importantly, these criteria are also needed to distinguish true PD from other parkinsonian syndromes (e.g., multiple system atrophy, progressive supranuclear palsy, corticobasal degeneration).

\subsubsection{Clinical Features}

Since the introduction of levodopa, the L-3,4-dihydroxyphenylalanine precursor of dopamine, in the1960s, the clinical picture and natural history of PD has changed drastically. The many problems facing the untreated PD patient before the advent of levodopa therapy have now been replaced with the serious complications of long-term levodopa treatment, such as choreiform and dystonic involuntary movements, motor fluctuations (the on-off phenomenon), orthostatic hypotension and confusional states with visual hallucinations. However, as was the case before the arrival of levodopa therapy, there remains a minority of patients with PD who will not respond to pharmacotherapy. With this in mind, it is common to distinguish between the primary (tremor, rigidity and bradykinesia) and secondary (dementia, depression, axiety, visuospatial deficits, blurred vision, dysarthria, dysphagia, impaired gastrointestinal motility, constipation and paresthesias) manifestations of PD.

\subsubsection{Tremor}

The tremor of PD results from an oscillation of agonist and antagonist muscles and is arguably the most visible signature of the disease. Usually noted in the early stages of the disease, tremor affects approximately 75 percent of PD patients (Hoehn \&

Yahr 1967). Tremor often subsides as a prominent sign late in the course of PD, as bradykinesia and postural instability become dominant features. The usual frequency of $\mathrm{PD}$ resting tremor is 3 to $5 \mathrm{~Hz}$. Physiologic tremor, in contrast, typically has a frequency of 8 to $10 \mathrm{~Hz}$ (Findley et al 1981). Characteristically, the tremor is noticed at rest and 
diminishes with purposeful movement. The rate of progression of tremor is highly variable, much like signs and symptoms of PD. It is most pronounced in the distal extremities, but at times may involve an entire limb. With advancing disease, the tremor can spread to involve the lips, jaw, chin and tongue.

The cause of tremor in the setting of PD remains unknown. Intracranial recordings in primates and human patients undergoing stereotactic neurosurgical procedures show that the ventral intermediate nucleus (VIM) of the thalamus contains intrinsic pacemaker neurons which fire at the same frequency as parkinsonian tremor (Ohye et al 1974; Ward et al 1948). Moreover, lesioning of a precisely selected portion of the VIM can abolish tremor, as can lesioning of the internal segment of the globus pallidus (GPi) (Benabid et al 1996; Taha et al 1996). Although the thalamus is likely the central generator of tremor in PD, it remains unclear as to how neuronal degeneration in the substantia nigra pars compacta (SNpc), the focal point of PD pathology, and the resultant depletion of dopamine influences other neurons in a particular nucleus of the thalamus to generate tremor. This conundrum is underscored by the observation that ablation of the SNpc and its striatal connections in experimental animals does not induce tremor.

\subsubsection{Rigidity}

Rigidity is defined as resistance to passive movement that occurs in both flexors and extensors throughout full range of motion. In contrast, spasticity is increased muscle tone present at initiation of movement which then quickly subsides ("clasped knife" phenomenon). Parkinsonian rigidity displays the classic "cogwheeling" quality, caused by the overlay of resting tremor on increased muscle tone even though the tremor may not be visible (Lance et al 1963). Cogwheeling is more apparent in the upper extremities, probably because tremor is also more prevalent in the arms as compared to the legs. Rigidity is present in the majority of PD patients, exhibiting great variation in severity and laterality. There is, however, a strong correlation between the severity of rigidity and the progression of disease, although the contribution of rigidity by itself to overall disability remains unclear (Selby 1984).

Although the pathophysiology of rigidity in PD is obscure, there is evidence that an abnormality of muscle spindle feedback to the spinal cord may contribute to symptomatology (Guekht et al 2005). However, primary muscle spindle afferent activity is normal in PD and there is some evidence to suggest hyperactivity of spinal interneurons or long loop reflex pathways with feedback to sensorimotor cortex in muscle rigidity (Cody et al 1986; Tatton et al 1984). In the brain, the GPi may exert influence on rigidity, as surgical lesioning in the posteroventrolateral segment abolishes parkinsonian rigidity in contralateral limbs (Laitinen et al 1992).

\subsubsection{Bradykinesia}

Bradykinesia is present in the majority of PD patients at initial presentation, even if rigidity and tremor are not apparent. Bradykinesia, defined as a slowness in movement or difficulty initiating movement, accounts for typical parkinsonian signs such

as decreased dexterity and the inability to perform rapid alternating movements. As the disease progresses, bradykinesia involving larger muscle groups is responsible for 
difficulty standing from a seated position, walking and turning in bed. Secondary symptoms such as masked facies (decreased range of facial expression), sialorrhea due to decreased frequency of swallowing and overall slowing of gastrointestinal motility are also attributed to bradykinesia. Dopamine depletion in the basal ganglionic-frontal cortical circuitry mediating motor programming and planning is the fundamental cause of bradykinesia (Marsden 1982). This is supported by the often dramatic response of bradykinesia to levodopa therapy in PD patients. It is believed that motor programming, (a cognitive function) is normal in PD, while the execution of planned motor tasks (also cognitive) is disrupted (Day et al 1984). This hypothesis is supported by the results of neuropsychological testing in PD patients revealing the loss of frontal executive ability, along with positron emission tomography imaging showing decreased activation of frontal premotor cortex during cognitive tasks (Jenkins et al 1992).

\subsubsection{Parkinsonian Gait}

The classic gait of PD, described as narrowly based, small-stepped and shuffling with a stooped-forward appearance with the arms held close to the body, was initially depicted in detail by James Parkinson. Patients may transiently hesitate in initiating the first steps, but move forward in steady fashion until a doorway or other obstacle causes them to slow, stop and freeze again until attempting to resume regular walking speed. Parkinson also describes the phenomenon of festination, or the tendency for the upper body to travel faster than the shuffling lower body, often resulting in a fall or collision. A complex motor activity, gait requires input from multiple levels of the nervous system, including the basal ganglia and spinal cord, as well as multiple functions such as proprioception, vision, coordination, muscle tone and vestibular equilibrium (Marsden 1982; Nutt et al 1993).

In the early stages of illness, the majority of PD patients have little to no difficulty with walking. In contrast, early-onset gait disorder associated with postural instability should arouse the suspicion of a parkinsonian sydrome other than PD, such as progressive supranuclear palsy or multisystem atrophy. In advanced PD, postural instability develops due to a loss of basal ganglia-mediated postural righting reflexes. Minor pertubations of balance can cause uncontrolled forward or backward movement, resulting in frequent falls and, at times, serious injury (Nutt et al 1993). It remains unclear why visual, vestibular and proprioceptive corrective reflexes fail to compensate for the postural instability in late PD. The worsening of this postural instability with advancing disease, even as levodopa therapy controls other symptoms, strongly suggests that non-dopaminergic systems and circuits outside of the basal ganglia may play a principal role.

\subsubsection{Secondary Manifestations}

Depression has long been recognized as a frequent and serious disorder in PD patients. The reported incidence of significant depressive symptoms in PD ranges from $30-40 \%$ (Reijnders et al 2008). Key characteristics of depression in PD are decreased mood and anhedonia. Other features of depression can be present in varying combinations but many of these, such as decreased appetite or sleep, weight change, psychomotor retardation, reduced memory and loss of energy can overlap with the symptoms of PD, making diagnosis of depression difficult. However, feelings of guilt or 
worthlessness and suicidal ideation may be less common in PD patients (Gotham et al 1986). The concept that depression should be viewed as an integral component of disease rather than a purely psychological reaction is supported by the observation that in $15-25 \%$ of cases, unexplained depression preceded the onset of motor symptoms of PD, often by as long as several years (Mayeux et al 1984b).

Cognitive dysfunction and dementia have long been recognized as causes of morbidity and disability in PD patients. Defined as the loss of cognitive and intellectual functions without impairment of perception or consciousness, an estimated $20 \%$ of PD patients suffer from dementia (Brown \& Marsden 1988). In 1974 the term "subcortical dementia" was introduced, with typical features of slowness of information processing, altered personality with apathy, forgetfulness and a decreased ability to retrieve acquired knowledge (Albert et al 1974). By definition, subcortical dementia lacks the cortical features of aphasia, apraxia and agnosia typically found in Alzheimer disease (AD), the classic prototype of cortical dementia. Recently, overlap between cortical and subcortical dementia has been emphasized, as has the utility of using neuropsychological data to classify the cognitive disorders associated with PD and parkinsonian syndromes (Pillon et al 1996). Deficits in visuospatial discrimination, frontal lobe executive function and memory retrieval are typical of the subcortical dementias in general and PD in particular (Hamill et al 1988). However, most data illustrate that the clinical distinction between cortical and subcortical dementia is at best arbitrary and the gold standard for accurate anatomical diagnosis remains histo- and immunopathology (Hansen \& Samuel 1997).

At least some degree of autonomic nervous system dysfunction is present in the majority of PD patients. Although in most the degree of impairment is mild, it can be the cause of significant functional disability. Low-normal blood pressure is very common in PD due to degeneration of sympathetic neurons of the hypothalamus. Dopaminergic agonists used in the treatment of PD have the potential to lower blood pressure further, often leading to orthostatic dizziness and syncope. Urinary urgency and urge incontinence occur in PD with degeneration of descending frontal cortical inhibitory pathways to the bladder, secondarily reducing its capacity and leading to spasticity (Berger et al 1987). Autonomic dysfunction of the gastrointestinal (GI) tract is almost universal in PD. Although an exact mechanism is unknown, degeneration of the myenteric plexus with a-synuclein immunoreactive Lewy body (LB) inclusions has been documented in patients at autopsy (Braak et al 2006; Qualman et al 1984). Slowed motility of the $\mathrm{Gl}$ tract may also lead to excessive salivation and poor anal contraction (Edwards et al 1992). Sialorrhea results from infrequent glutition, however, rather than overproduction. Likewise, segmental esophageal spasm is a result of poor motility and most patients experiencing dysphagia swallow soft foods and liquids without difficulty (Gibberd et al 1974). Slow gastric and small bowel motility translates into subjective feelings of postprandial fullness and bloating, sometimes to the point of pseudoobstruction. Constipation can be a pervasive source of discontent in PD patients and is usually relieved only with a carefully designed regimen of dietary measures, stool softeners and laxatives. Obstipation and fecal impaction are common outcomes when constipation is neglected or not aggressively managed (Jost 2010).

In addition to dysfunction of the motor system, up to 50\% of PD patients report sensory disturbances of some form (Snider et al 1976). For instance, paresthesias or burning pain in the limbs may precede the appearance of the more clinically recognizable signs of disease by months or even years and are rarely associated with 
objective findings on neurological exam (Quinn et al 1986). Restlessness of the legs or body and other more bizarre sensations also may be common manifestations of the "wearing-off" effect of levodopa therapy (Nutt \& Carter 1984). The loss of olfactory sense is a frequent and poorly understood defect in PD, often demonstrable at or even before the onset of motor symptoms (Doty et al 1992). A similar finding is commonly seen in the early stages of $A D$ which may suggest a commonality in neurodegenerative pathogenesis.

Disruption of the normal sleep cycle is almost universal in advanced PD. The most common pattern, especially late in the course of disease, is fitful sleep at night and excessive napping during the day. Excessive daytime sleeping becomes even more problematic for the patient when it occupies so much of the day that worthwhile physical and social activities are thwarted. Disrupted sleep has many causes, including poor bed mobility due to rigidity and bradykinesia, nocturnal urinary urgency and frequency, and vivid dreaming and early morning awakening secondary to depression (Askenasy 1981). Patients with significant cognitive loss or dementia tend to sleep excessively, day and night. However, alarming periods of agitation and even belligerent behavior can erupt after sundown or at unpredictable times during the night.

Hypophonia, the loss of voice volume, is the most common manifestation of parkinsonian speech impairment. Slowed movement and incoordination of the muscles of speech production, within the lips, tongue and pharynx, muffle the normally sharp articulation of speech. As bradykinesia worsens, voice volume may decline further and speech output takes on a festinating quality as the repetitive movements of the involved oropharyngeal muscles cause words to run together. As with any sustained, repetitive movement in PD, amplitude steadily decreases in direct relation to the duration of the activity. Therefore, relatively forceful enunciation at the beginning of speech production is rapidly followed by gradual loss of volume and speech quality. As may be expected, vocal cord rigidity is a common laryngoscopic finding in PD patients, contributing to hoarseness, changes in pitch and other abnormalities of prosody (Vincken et al 1984).

\subsubsection{Natural History}

Longitudinal studies of the natural history of PD reveal a long course of illness, remarkably variable and unpredictable, with most patients experiencing all of the classic features in the earliest stages. Clinical disability is by nature progressive due to the increasing loss of nigral neurons over time. However, factors determining the tempo of cell death in the individual are currently unknown (Hughes et al 1993). Because the pace of pathologic progression is slow in the majority of patients, those with levodopa responsive parkinsonism may function independently if not normally for many years after initial symptoms appear. However, some deteriorate more rapidly and lose all independence within a few years due to medically intractable bradykinesia, postural instability and cognitive impairment. Furthermore, some patients in whom tremor is an early and dominant symptom remain unencumbered by other symptoms of PD before signs of true progression occur. In contrast, early onset postural instability and gait disorder (PIGD) usually predicts an accelerated decline in function and is a predictor that a non-LB Parkinsonian syndrome may underlie the cause of disability, especially if the response to levodopa is weak. Although PD is typically a disorder of middle and older age, those of younger age (less than 40 years) can be affected but often experience a more benign course with a better response to levodopa than the elderly despite the 
dyskinesias and motor fluctuations that younger patients may encounter with chronic levodopa therapy.

The impact of levodopa on the natural history of PD has been mixed. Although the benefit of levodopa to the patient cannot be overestimated, the practical and hypothetical problems associaed with long-term treatment have not been trivial. Morbidity and mortality have dropped substantially since the pre-levodopa era, when drug therapy consisted of relatively ineffectual agents with low therapeutic margins (Diamond \& Markham 1976). However, data accumulated over the last 30 years have shown that despite the vastly improved quality of life for the average PD patient, progression of disability is no less variable than before. Furthermore, although mortality has been reduced in the levodopa era compared with the years before, death rates remain higher in PD patients versus age-matched controls (Morens et al 1996).

Long term levodopa use has been associated with a variety of adverse cognitive and motor consequences. The early period of a sustained and excellant response to levodopa usually gives way unpredictably after several years to motor fluctuations, characterized by a distinct feeling of activation ("turning on") 20-30 minutes after taking the drug, followed by a wearing off of effectiveness 3-6 hours later and a return of bradykinesia. The usual dosing schedule of three to four times a day that once worked well to provide a smooth and unvarying response must be replaced by more frequent dosing to accommodate the emergence of the short-duration response. The cause of these "on" and "off" cycles is presumed to be the result of an incompletely understood combination of diminished capacity of the nigrostriatal neurons to store and tonically release dopamine derived from exogenous levodopa and of downregulation of postsynaptic striatal dopaminergic receptors (Hurtig 1997). Dyskinesias or abnormal involuntary movements of the extremities gradually appear during the evolution of motor fluctuations, occurring most commonly during the pharmacokinetic peak of each dose of levodopa (Nutt 1990). As parkinsonian disability increases with progressive loss of nigral neurons, so does the severity of on-off fluctuations and dyskinesias. Treatment of fluctuations with a combination of dopamine agonists and levodopa can potentially modulate fluctuations by allowing a reduction in levodopa dose without a loss of benefit. The use of a controlled-release combination of levodopa and carbidopa (a peripheral dopa decarboxylase inhibitor) can dampen the intensity of dyskinesias and lengthen the duration of motor response.

Dyskinesias usually have distinctively choreoathetotic features, but may also contain elements of dystonic posturing, rapid myoclonic jerking or both (Luquin et al 1992; Nutt 1990). Dyskinesias occur most commonly at the peak of the dose-response cycle, but levodopa's short half-life $(90 \mathrm{~min})$ limits the duration of any dyskinetic reaction unless dosing is so frequent that blood levels become markedly elevated. Dyskinesias can also take the form of dystonic posturing of the extremities, occuring during the wearing-off phase. Dystonic posturing on the side of greater Parkinsonian impairment is a common early-morning off reaction in some patients after doing without levodopa overnight. This usually resolves after the first dose of levodopa of the day. In contrast with choreoathetotic involuntary movements, dystonic posturing of the hands, feet and neck can occur as an integral symptom of untreated parkinsonism (Muenter et al 1977).

Parkinsonism is a chronic and progressive disease with decreased life expectancy despite modern treatment. Hoehn and Yahr first reported a standardized mortality ratio (the ratio of observed deaths to expected deaths) of 2.9 in patients with 
PD (Hoehn \& Yahr 1967). Standardized mortality ratios from more recent PD studies have ranged from 1.3 to 4.1 (Herlofson et al 2004; Marras et al 2005). In a study of PD patients in the midwestern United States, median survival of patients from diagnosis was 10.3 years, while in the Sydney Multicenter Study median time from motor onset to death was 12.2 years (Elbaz et al 2003; Hely et al 2005). In these studies, disease severity, dementia and age were independent risk factors for increased mortality in PD. However, the cause of death in PD patients is often related to fatal infections as a result of immobility (Beyer et al 2001).

\subsubsection{Epidemiology}

The term "Parkinsonian syndrome" (PS) refers to a condition in which at least two of the three major clinical manifestations associated with PD are found (Rajput et al 1991). Therefore, this condition includes several different entities. In some cases the cause is recognizable: postencephalitic parkinsonism, posttraumatic parkinsonism, neuroleptic drug-induced parkinsonism, $N$-methyl-4-phenyl-1,2,3,6-tetrahydropyridine (MPTP) toxicity, manganese toxicity and cyanide poisoning (Calne et al 1994; Casson et al 1984; Duvoisin \& Yahr 1965; Langston et al 1983; Uitti et al 1985). However, in the majority of cases the cause is often unknown. The most common form of parkinsonism is idiopathic PD (Duvoisin \& Golbe 1989). PS is also a feature of several other degenerative diseases, such as progressive supranuclear palsy, mutiple system atrophy, Creutzfeld-Jakob disease and corticobasal degeneration.

Because a clinical assessment may not accurately distinguish between different PS variants, studies that include only the diagnosis of PD likely include a significant proportion of other PS variants. Community epidemiologic prevalence surveys are usually based on a single neurological assessment and may include false-positive diagnoses of PD as high as 35\%, as reported in autopsy verification of initial PD diagnosis by neurologists (Bharucha et al 1988; Morgante et al 1992; Schoenberg et al 1985). To reduce such error, Hughes and colleagues proposed a different set of diagnostic criteria: asymmetrical symptom onset and no evidence of another disorder or possible etiology (Hughes et al 1992). When applying these criteria to their own autopsied series, they found that $7 \%$ of the non-PD cases were misdiagnosed as PD and 32 percent of PD cases did not fit their criteria. In addition, further autopsy studies have shown that approximately $4 \%$ of PD cases have a symmetrical onset of symptoms (Rajput et al 1993). Therefore, the proposed criteria of Hughes et al may not suitable for descriptive epidemiology. In turn, studies that include a liberal definition of PS diagnosis are liable to include non-PS cases and the distinction between normal aging and early PS may be difficult.

\subsubsection{Incidence}

Incidence calculations are thought to be a more precise estimate of the frequency of disase as they are not influenced by mortality. Published age-standardized incidence rates for parkinsonism range from 9 to 20 per 100,000 population in the United States (US) and European countries (Twelves et al 2003). Some studies may include all PS, while others are restricted to PD cases. The lowest incidence in the western world is 4.9 per 100,000, reported from Sardinia between 1961 and 1971 (Rosati et al 1980). A report from the Netherlands noted an incidence rate of 11 per 100,000 for men and 12 
per 100,000 for women (Hofman et al 1989). In Japan, Harada and colleagues reported annual incidence of 10.2 per 100,000 population (Harada et al 1983).

All literature reports have noted a progressive increase in the incidence of PD with age (Mayeux et al 1995; Rajput \& Birdi 1997). Onset before age 30 is very rare and onset before age 40 accounts for 4 to $10 \%$ of all cases of PS (Rajput 1992). After age 50 , age-specific incidence rises dramatically. Data from the Mayo Clinic in Rochester, MN, between the years of 1935 and 1979 show that between the ages of 40 and 49 the incidence of PD was 5 per 100,000; this value increased 35-fold to 174 per 100,000 in the 70 to 79-year-old age group (Rajput et al 1984).

Gender differences in the development of PD have been described in numerous studies, most of which report higher incidence rates in males, especially in the oldest age groups (Bharucha et al 1988; Diamond et al 1990; Kessler 1972; Schoenberg et al 1985; Taylor et al 2007; Vanacore et al 1992). However, several studies have found no difference in the incidence of PD between men and women less than 60 years of age. Neuroprotective properties of female steroid hormones and gender differences in exposure to environmental or occupational risk factors have been discussed as underlying causes (Shulman 2007; Taylor et al 2007). In a meta-analysis of 17 incidence studies, the overall age-adjusted male to female ratio for incident PD was 1.46 (Shulman 2007; Taylor et al 2007). However, there is some data to suggest that gender incidence may differ by ethnicity, with a higher incidence of male PD in western populations.

\subsubsection{Prevalence}

The prevalence rate of PD can be determined by several different methods, the most reliable of which is that based on the door-to-door community survey (Bharucha et al 1988; Morgante et al 1992; Schoenberg et al 1985). It is estimated that 35 to $42 \%$ of PD cases in the community are undiagnosed, thus community surveys yield higher and presumably more accurate rates than other methods (Morgante et al 1992; Schoenberg et al 1985). PD is found in all ethnic groups, with geographical differences in prevalence. Early-onset sporadic PD is rare, with about $4 \%$ of patients developing clinical signs of disease before age 50 (Van Den Eeden et al 2003). Approximately 1 to $2 \%$ of the population over age 65 suffers from PD. This figure increases to 3 to $5 \%$ in those age 85 or older (Fahn 2003). As PD is primarily an illness of later life, it is more common in developed countries where life expectancy is longer. Door-to-door surveys report prevalence rates of PD ranging from 57 per 100,000 in China to 371.5 per 100,000 in Sicily (Li et al 1985; Morgante et al 1992). Most communtiy-based prevalence studies across Europe found prevalence rates between 100 and 200 per 100,000 inhabitants (von Campenhausen et al 2005). A prevalence of 328 per 100,000 was reported in a community from Bombay, India, and 347 per 100,000 in Copiah County, Mississippi (Bharucha et al 1988; Schoenberg et al 1985). Overall, the estimated prevalence rate of PD in the United States and Canada is approximately 300 per 100,000 (Duvoisin \& Golbe 1989). 


\subsubsection{Geographic Environments}

The variation in prevalence rate of PD across geographic enviornments is notable and warrants further discussion. The lowest reported prevalence rate, 57 per 100,000 , is found in China, followed by Sardina (65.6 per 100,000), Nigeria (67 per 100,000), and Japan (80.6 per 100,000) (Harada et al 1983; Li et al 1985; Rosati et al 1980; Schoenberg et al 1988). The highest prevalence rate reported, 371.5 per 100,000 , is found in Sicily (Morgante et al 1992). Different prevalence rates have also been reported in populations of the same race. Northampton, England, has a prevalence rate of 108 per 100,000, and that of Aberdeen, Scotland is 164.2 per 100,000 (Schoenberg 1987). Such differences can be in part attributed to variations in methods. However, two studies by Schoenberg and colleagues used identical methods and found markedly different prevalence rates in populations of the same race (Schoenberg et al 1985; Schoenberg et al 1988). Although African-Americans and Caucasians in the US had similar prevalence rates, the rates for US African-Americans was fivefold higher than that of Africans in Nigeria (Schoenberg et al 1988). This finding indicates a genuine difference based on geography.

Differences in the incidence of PD based on population density were first reported in Saskatchewan, Canada, and it was concluded that those born and raised in rural Saskatchewan had an increased risk of developing early-onset PD (Rajput et al 1986). In this study, a rural community was defined as one which utilized well water as a drinking source. Interestingly, 19 out of 21 patients in this study utilized well water exclusively for the first 15 years of life. Along similar lines of rural living, Barbeau and colleagues reported a higher PD concentration in communities that concentrated on market gardening (Barbeau et al 1987). In addition, several other North American and European reports noted a higher PD risk associated with prior rural residence (Hertzman et al 1990; Hubble et al 1993; Koller et al 1990; Ludin \& Ludin 1989; Tanner et al 1987). For instance, Butterfield and colleagues noted that rural residency in Oregon at the time of diagnosis, as well as nut and seed eating 10 years prior to dagnosis was more common in PD cases than in age-matched controls (Butterfield et al 1993). Although an increased risk of PD has been associated with exposure to fertilizers, pesticides, insecticides, paints, wood preservatives and fungicides, no specific chemical or chemicals have been attributed to date (Barbeau et al 1987; Hertzman et al 1990; Koller et al 1990; Seidler et al 1996; Semchuk et al 1991; 1992; Svenson 1991; Svenson et al 1993; Tanner et al 1989).

\subsubsection{Genetic Risk Factors}

In recent years, genetic risk factors have become increasingly important in the search for possible causes of PD. Family members of affected patients are at three- to four-fold increased risk of developing PD compared to the general population (Autere et al 2000; Kurz et al 2003). The first finding strengthening the involvement of genetic risk factors in PD was the identificaiton of the PARK1 locus on chromosome $4 q 21$, linking to familial forms of the disease (Polymeropoulos et al 1996). The finding of a chromosomal region linked to $P D$ was later confirmed by identification of the point mutation A53T in the SNCA ( $\alpha$-synuclein) gene in Italian and Greek families with autosomal dominant inheritance (Polymeropoulos et al 1997). During the last two decades, other gene loci were found to be associated with autosomal dominantly ( $\alpha$-synuclein [PARK4] and

ubiquitin carboxyl-terminal esterase L1 [PARK5]) or recessively (parkin [PARK2], PTEN 
induced putative kinase 1 [PINK1 or PARK6], DJ-1 [PARK7] and ATPase 13A2 [PARK9]) inherited parkinsonism (Gasser et al 1998; Hampshire et al 2001; Kitada et al 1998; Leroy et al 1998; Paisan-Ruiz et al 2004; Singleton et al 2003; Valente et al 2004; Valente et al 2001; van Duijn et al 2001). To date, most of the known gene mutations cause juvenile or early onset of the disease, while others such as the most recently identified leucine-rich repeat kinase 2 (LRRK2 or PARK8) mutation and several mutations in glucocerebrosidase (GBA) appear to cause parkinsonism resembling sporadic PD with respect to both clinical and demographical features (Aasly et al 2005; Funayama et al 2002; Sidransky et al 2009). The discovery of these genetic mutations and the increased understanding in dysfunction of their aberrantly encoded proteins has provided important and novel insights into the molecular pathogenesis of the disease. There is now strong evidence that impairment of the ubiquitin-proteasome system, mitochondrial dysfunction and decreased oxidative stress tolerance are key mechanisms in PD pathogenesis (Eriksen et al 2005; Gandhi \& Wood 2005). However, the exact mechanisms are not completely understood and monogenetic causes only account for a small proportion ( $<10 \%$ ) of all PD cases, while the vast majority of PD cases appear to be sporadic. In these, occupational, lifestyle and environmental factors, possibly in interaction with each other or with susceptibility genes, may play a part.

\subsubsection{Cellular and Molecular Pathology}

The hallmark neuropathologic features of PD that are essential for the diagnosis of definite PD at postmortem brain examination include the extensive loss of dopaminergic neurons in the SNpc, the presence of intracytoplasmic inclusions (LBs) in the remaining dopaminergic neurons, variable amounts of extracellular melanin released from degenerated neurons and gliosis (Fearnley \& Lees 1991; Forno 1996; McGeer et al 1988). The primary neurochemical defect in PD is the loss of dopaminergic projections to the striatum as a result of the massive degeneration of the neuromelanin-containing dopaminergic neurons of the SNpc (Bernheimer et al 1973; Kish et al 1988). In addition to dopaminergic neurons in the SNpc, other populations of neuromelanin and nonneuromelanin containing neurons in the brainstem and basal forebrain degenerate and accumulate LBs that are similar to those seen in the SNpc (Forno 1996). Moreover, the loss of these populations of neurons leads to neurotransmitter deficits, but to a lesser extent than that which accompanies the degeneration of dopaminergic SNpc neurons. For example, norepinephrine, serotonin and acetylcholine are variably decreased in PD owing to the loss of neurons in the locus ceruleus, raphe nucleus and nucleus basalis of Maynert, respectively, and many of the secondary clinical features of PD have been ascribed to these neurotransmitter deficits (D'Amato et al 1987; Mayeux et al 1984a; Whitehouse 1987).

The LB is considered the signature histopathologic hallmark of PD (Forno 1996; Pollanen et al 1993). LBs were originally described in the cholinergic neurons of the substantia innominata by Friederich Lewy in 1912 and have subsequently been noted in the dopaminergic neurons of the SNpc in virtually all PD cases in association with neuronal loss and gliosis (Forno 1996; Hughes et al 1993). Furthermore, LBs are also seen in all other populations of neurons that degenerate in the PD brain and may be found in the cerebral cortex as well as in brainstem nuclei (Forno 1996; Galvin et al 1999). 
The morphology of the LB in PD is well established at the light- and electronmicroscopic levels. The majority of LBs in SNpc neurons exhibit a loose, radiating array of filaments in the periphery of the LB, surrounding a matted meshwork of filaments in the center or core (Forno 1996; Hill et al 1991; Pollanen et al 1993; Schmidt et al 1991). Although most LBs are spherical inclusions in the cell body of neurons, they may also assume an irregular geometry and extend into the proximal segments of neuronal processes. Most cellular organelles are excluded from LBs, which are often displaced or deformed along with the nucleus. The predominant structural elements in LBs are filaments 7 to $25 \mathrm{~nm}$ in diameter (Hill et al 1991; Schmidt et al 1991). In contrast to SNpc LBs, cortical LBs have a less stereotyped morphology that lacks a clearly definable central core and a distinct peripheral corona, which may account for the inconspicuous appearance by hematoxylin and eosin staining (Forno 1996; Galvin et al 1999; Okazaki et al 1961; Pollanen et al 1993; Schmidt et al 1991).

Much of the information currently known about the components of LBs has been obtained using immunohistochemical techniques. These studies have demonstrated that the major antigenic components of LBs are the presynaptic protein $\alpha$-synuclein, neurofilament proteins and ubiquitin, a protein involved in the non-lysosomal intracellular degradation of cellular products (Baba et al 1998; Forno et al 1986; Galvin et al 1997; Goldman \& Yen 1986; Goldman et al 1983; Irizarry et al 1998; Kuzuhara et al 1988; Lowe et al 1988; Lowe et al 1990; Spillantini et al 1998; Spillantini et al 1997). However, a number of other cytoskeletal and non-cytoskeletal proteins have also been identified within LBs. It has been suggested that the antigenic components of LBs can be divided into four groups: (1) structural elements of LB filaments; (2) proteins that reflect a cellular response to LB formation; (3) enzymes such as kinases and phosphatases, which may modulate LB filaments; and (4) other cytosolic proteins that presumably become trapped in LBs during formation of these inclusions (Pollanen et al 1993).

Although a large number of molecules have been identified in LBs, the most prominent is $\alpha$-synuclein. $\alpha$-synuclein was first cloned from the Torpedo californium and later identified in humans as the non-A $\beta$ component of AD amyloid (NAC) precursor (NACP) (Maroteaux et al 1988; Ueda et al 1993). Attention focused on a-synuclein when Polymeropoulos and colleagues reported that in a kindred with familial PD, a mutation, A53T, was associated with disease (Polymeropoulos et al 1996; Polymeropoulos et al 1997). A number of groups then quickly reported $\alpha$-synuclein as a major component of LBs (Baba et al 1998; Irizarry et al 1998; Spillantini et al 1997; Takeda et al 1998; Wakabayashi et al 1997). a-synuclein is a member of a highly conserved family of proteins consisting of $\alpha-, \beta$-, and $\gamma$-synuclein. The protein consists of 140 amino acids and the $\alpha$-synuclein gene contains seven exons, five of which are protein coding (Clayton \& George 1998; Norris et al 2004). The N-terminal 89 amino acids are composed almost entirely of six variants of a degenerate 11-amino acid consensus motif with slight variations. The 11-mer repeats comprise an apolipoproteinlike-class- $\mathrm{A}_{2}$ helix, which mediates binding to vesicles of phospholipids. In neutral solution, $\alpha$-synuclein is unfolded, but binding to lipid shifts the protein secondary structure from $\approx 3 \%$ to $>70 \%$ a-helix. The central region contains two of the 11-mer repeats and the hydrophobic NAC portion between residues 61 and 95 . The NAC region of $\alpha$-synuclein appears to be crucial to fibrillation, because $\alpha$-synuclein lacking the domain and $\beta$-synuclein, which lacks 11 amino acids in the NAC region, have reduced susceptibility to form fibrils. The $\mathrm{C}$ terminus is less conserved than the $\mathrm{N}$ terminus and contains a preponderance of acidic residues. 
The function(s) of $\alpha$-synuclein is not fully understood, but studies of $\alpha$-synuclein knockout (KO) mice have been informative. Abeliovich and colleagues reported that $\alpha-$ synuclein KO mice were viable and fertile and had normal brain architecture, particularly of the nigrostriatal dopaminergic system, indicating that $\alpha$-synuclein is not necessary for its normal development (Abeliovich et al 2000). The dopamine content in the striatum, but not the midbrain, was reduced. Open field activity was normal, but amphetamineinduced locomotion was reduced. Simple electrical stimulation of the nigrostriatal dopaminergic system produced a normal pattern of dopamine discharge and reuptake. Paired-pulse stimulation had accelerated recovery of release of dopamine after an initial stimulus, suggesting an inhibitory role for a-synuclein in the activity-dependent modulation of nigrostriatal dopamine transmission. Chandra and colleagues used single and double $\alpha$ - and $\beta$-synuclein KO mice and reported that the synucleins are not crucial to neurotransmitter release but may be involved in long-term regulation and/or maintenance of presynaptic function (Chandra et al 2004). Cabin and colleagues carried out similar studies in $\alpha$-synuclein KO mice and noted a reduction in the pool of undocked vesicles in the hippocampus and that prolonged stimulation in slices, which would rely on the reserve vesicle pool, was impaired (Cabin et al 2002). In cultured hippocampal cells, long-lasting potentiation of synaptic transmission between cells was accompanied by an increase in the number of $\alpha$-synuclein clusters. In cultures derived from $\alpha-$ synuclein KO mice this potentiation was not observed (Liu et al 2004). It was thus concluded that $\alpha$-synuclein is required for the genesis, localization and/or maintenance of at least some of the vesicles that make up the reserve or resting pools of presynaptic vesicles, and the data appear to be consistent with this conclusion (Cabin et al 2002). The mechanism by which $\alpha$-synuclein modulates neurotransmitter release is uncertain, but it may be via inhibition of phospholipase D2, with effects on levels of diacyl glycerol and phosphatidic acid and their effects on the size and recovery rate of the readily releasable pool of synaptic vesicles and membrane trafficking (Abeliovich et al 2000). Of particular interest, the $\alpha$-synuclein $\mathrm{KO}$ mice have shed light on certain pathogenic mechanisms important in PD. Four lines of $\alpha$-synuclein KO mice have demonstrated resistance to the neurotoxin MPTP, and the protective effect appeared to be upstream of the mitochondria (Dauer et al 2002).

Attention was drawn to the ubiquitin-proteasome system (UPS) by the discoveries that a common form of autosomal recessive PD is due to mutations in the Parkin gene, which encodes for an E3 ligase (Kitada et al 1998; Shimura et al 2000). Another member of the UPS, ubiquitin C-terminal hydroxylase L1, was reported to have a missense polymorphism in two siblings with PD (Leroy et al 1998). However, the role of the UPS and Parkin in the degradation of $\alpha$-synuclein is not fully understood. Bennett and colleagues reported that $\alpha$-synuclein was degraded by the UPS, but other groups have reported that inhibition of proteasomes did not increase levels of $\alpha$-synuclein, suggesting alternate pathways for degradation (Ancolio et al 2000; Bennett et al 1999; Rideout et al 2001). In vitro a-synuclein can be degraded by the $20 \mathrm{~S}$ proteasome through a ubiquitin-independent mechanism (Tofaris et al 2001). Infusion of the proteasome inhibitor lactacystin into the $\mathrm{SN}$ and lactacystin and epoxomycin (a naturally occurring inhibitor) into the striatum created models of PD in the rat (Fornai et al 2003; McNaught et al 2002). McNaught and colleagues extended this data by systemically administering epoxomicin to rats and found that the animals developed behavior and pathology with $\alpha$-synuclein inclusions reminiscent of PD (McNaught et al 2004). However, mutant forms of $\alpha$-synuclein and protein aggregates have been shown to reduce proteasomal activity (Bence et al 2001; Petrucelli et al 2002; Stefanis et al 2001; Tanaka et al 2001). The finding that Parkin KO mice have a normal level of $\alpha$-synuclein 
raised the possibility that it is not a normal substrate for Parkin and that other degradation systems are involved (Goldberg et al 2003). Inhibition of both the proteasome and autophagy increased the levels of $\alpha$-synuclein in PC12 cells with $\alpha-$ synuclein in autophagic vacuoles and lysosomes (Webb et al 2003). In cultured cortical neurons, proteasome inhibition led to activation of macroautophagy and the lysosomal pathway (Rideout et al 2004). In cultured neuronal cells, $\alpha$-synuclein oligomers, but not mature fibrillar inclusions, were cleared by the lysosomal pathway (Lee et al 2004a). Cuervo and colleagues reported that wild type (WT) a-synuclein was internalized and degraded in lysosomes by chaperone-mediated autophagy, and A30P and A53T forms of $\alpha$-synuclein blocked lysosomal uptake and degradation of $\alpha$-synuclein by chaperonemediated autophagy (Cuervo et al 2004). Of note, four groups have reported an increased prevalence of heterozygous carriers of mutant forms of GBA, the lysosomal enzyme involved in Gaucher's disease, in Parkinsonian patients, and one series described cortical and/or hippocampal LBs (Aharon-Peretz et al 2004; Clark et al 2005; Goker-Alpan et al 2004; Lwin et al 2004; Sato et al 2005). Subclinical lysosomal dysfunction in cases with mutant GBA has thus been hypothesized to lead to impaired clearance of $\alpha$-synuclein (Goker-Alpan et al 2004). This data indicates that $\alpha$-synuclein can be degraded by both the proteasome (through ubiquitin-dependent and independent processes) and autophagy.

A number of groups have demonstrated that $\alpha$-synuclein can aggregate to form protofibrils and amyloid-like fibrillar structures (Conway et al 1998; Giasson et al 1999; Hashimoto et al 1998). In solution, the mutations A53T and A30P increased the rate of $\alpha$-synuclein oligomerization and formation of $\beta$-sheets (Narhi et al 1999; Uversky \& Fink 2002). The rate of formation of mature fibrils was increased by A53T and reduced by A30P (Conway et al 2000). Overexpression of $\alpha$-synuclein in a number of cell lines resulted in the formation of aggregates (Hsu et al 2000; Stefanis et al 2001; Stefanova et al 2001). Masliah and colleagues overexpressed WT a-synuclein in mice and found amorphous and granular (but not fibrillar) aggregates of $\alpha$-synuclein in various brain regions in which LBs are found (Masliah et al 2000). The mice exhibited injury to the nigrostriatal dopaminergic system and motor deficits. Adenoviral vector-selective overexpression of WT and A53T $\alpha$-synuclein in the SN in nonhuman primates caused $\alpha-$ synuclein inclusions and degeneration of dopaminergic neurons (Kirik et al 2003). Overexpression of WT and mutant a-synuclein in Drosophila resulted in intraneuronal $\alpha$ synuclein inclusions, loss of dopaminergic neurons and motor deficits (Feany \& Bender 2000). Overexpression of mutant and WT a-synuclein in Caenorhabditis elegans resulted in dopaminergic cell loss as well (Lakso et al 2003). The concentration dependence of aggregation may be relevant to PD, because triplication of the $\alpha-$ synuclein gene is associated with familial PD (Singleton et al 2003).

Evidence of excessive oxidation is found in the brains of PD patients (Alam et al 1997a; Alam et al 1997b; Dexter et al 1989). Hashimoto and colleagues demonstrated that treatment of $\alpha$-synuclein with $\mathrm{H}_{2} \mathrm{O}_{2}$ and ferrous iron in solution led to the formation of insoluble aggregates of $\alpha$-synuclein (Hashimoto et al 1999). Treatment of neuroblastoma cells overexpressing A53T, A30P and WT a-synuclein with iron resulted in the formation of $\alpha$-synuclein aggregates, and the mutants were found to be more susceptible (Ostrerova-Golts et al 2000). Furthermore, increased amounts of iron have been found in the SN of PD brains (Dexter et al 1991). Considerable evidence exists that mitochondrial dysfunction and oxidative stress are involved in the pathogenesis of PD. Chronic treatment of rats with rotenone, a known complex I mitochondrial inhibitor, resulted in injury to the nigrostriatal dopaminergic system and formation of a-synuclein 
aggregates and continuous exposure of mice to MPTP resulted in a PD phenotype and formation of LB-like inclusions (Betarbet et al 2000; Fornai et al 2005). a-synuclein inclusions in brains of PD patients have also been shown to contain oxidized $\alpha$-synuclein (Duda et al 2002).

The observation that up to $50 \%$ of $A D$ cases also contain LBs suggests there might be a commonality in the processes that form LBs and the senile plaques and neurofibrillary tangles (NFTs), the pathological hallmarks of AD (Hansen et al 1990; Kotzbauer et al 2004; Lee et al 2004b). Cases of familial AD can have LBs, and patients with the A53T mutation in the gene for $\alpha$-synuclein have tau, as well as $\alpha$-synuclein, inclusions (Kotzbauer et al 2004; Lippa et al 1998). Tau proteins interact with tubulin to stabilize microtubules and promote tubulin assembly. Six tau isoforms exist in brain tissue and they are distinguished by their number of binding domains. Giasson and colleagues reported that $\alpha$-synuclein could initiate polymerization of tau in vitro, and that tau significantly increased the assembly of $\alpha$-synuclein into fibrils (Giasson et al 2003). However, both tau and $\alpha$-synuclein preferentially form homopolymers rather than heteropolymers, suggesting that each may participate in an initiation phase of formation of fibrils, but that enlargement into larger fibrils is specific for each protein and that these and other amyloidgenic proteins can induce both $\alpha$-synuclein and tau pathology (Geddes 2005; Lee et al 2004b). In AD, neurons tend show a predilection to form NFTs or LBs, and tau and $\alpha$-synuclein tend to segregate into separate filaments (Geddes 2005). This commonality might also be due to processes that underlie the formation of tau and $\alpha$ synuclein inclusions.

It is plausible that excessive amounts of $\alpha$-synuclein oligomers might overwhelm the mechanisms responsible for clearance of aberrant proteins, thereby interfering with the normal physiology of the cell and eventually leading to cell injury and death. Impairment of proteasome function can obviously be the primary event (as occurs in patients who have mutations in the Parkin gene but lack LBs), but investigators have also shown that mutant forms of $\alpha$-synuclein and protein aggregates can reduce proteasomal activity (Bence et al 2001; Petrucelli et al 2002; Stefanis et al 2001; Tanaka et al 2001). The inability to normally degrade misfolded and damaged proteins would lead to increasing dysfunction and death of cells. As discussed above, there is evidence of proteasomal dysfunction in PD and animal models in which proteasome inhibitors have been reported to create the behavioral and pathological features of PD. Interestingly, Parkin KO mice exhibited mitochondrial dysfunction and oxidative damage (Palacino et al 2004). Mitochondrial dysfunction has been established to occur in PD and overexpression of $\alpha$-synuclein has been reported to impair mitochondrial function (Hsu et al 2000).

Specific mechanisms through which $\alpha$-synuclein aggregates injure neurons have not been identified. Our current understanding of the pathogenic mechanisms in PD involves three pathways: protein aggregation; impaired protein degradation (i.e., dysfunction of the proteasome and lysosome systems); and mitochondrial dysfunction/oxidative stress (Betarbet et al 2000; Cuervo et al 2004; Dawson \& Dawson 2003; Eriksen et al 2003; Ross \& Pickart 2004; Shults 2004; Snyder \& Wolozin 2004). Other processes, such as inflammation, may also contribute to the death of cells (Hald \& Lotharius 2005; Teismann \& Schulz 2004). These three processes likely overlap and interact (Dawson \& Dawson 2003; Eriksen et al 2003; Greenamyre \& Hastings 2004). For example, overexpression and aggregation of $\alpha$-synuclein can lead to mitochondrial dysfunction, and inhibition of mitochondrial function with MPTP and rotenone leads to 
aggregation of $\alpha$-synuclein (Betarbet et al 2000; Fornai et al 2005; Hsu et al 2000). Impairment of the proteasomal system in Parkin KO mice and in PC12 cells leads to mitochondrial dysfunction and mitochondrial dysfunction results in impairment of proteasomal function (Hoglinger et al 2003; Lee et al 2005; Palacino et al 2004). Inhibition of the proteasomal system results in formation of $\alpha$-synuclein aggregates and aggregates of $\alpha$-synuclein can impair proteasomal function (Bence et al 2001; Fornai et al 2003; McNaught et al 2002; McNaught et al 2004). Malfunction of mitochondria or the proteasome and lysosome systems would result in the accumulation of proteins, many abnormal, and obviously would interfere with function of the cell. However, specific mechanisms through which these aggregates of proteins are injurious to cells have been surprisingly unexplored. The processes involved in the formation of LBs suggest a mechanism through which these processes may interact, lead to $\alpha$-synuclein aggregation, and result in mitochondrial dysfunction and potentially release of proapoptotic molecules. It may not be coincidence that mitochondria are a conspicuous component of LB (Gai et al 2000; Hayashida et al 1993; Roy \& Wolman 1969). Mitochondria, which both produces ATP for the cell and are central to apoptosis, are composed of an outer membrane, an intermembrane space and an inner membrane and matrix (Shults 2004; Wallace 2005). The electron transport chain (ETC), which is composed of five complexes, is located on the inner mitochondrial membrane, and energy released by passage of electrons down the ETC is used by complexes I, III, and IV to transport protons from the matrix to the intermembrane space. The transport of protons creates a proton and electrochemical gradient across the inner membrane, and the energy stored in the electrochemical proton gradient is used to drive complex $V$ to form ATP. In addition to its role in the bioenergetics of the cell, or perhaps because of its central position in the energetics of the cell, the mitochondrion has evolved to play a central role in apoptosis through the release of proapoptotic molecules from the mitochondrial intermembrane space (Green 2005; Green \& Kroemer 2004; Youle \& Karbowski 2005).

\subsection{THE TETRAHYDROISOQUINOLINES}

After systemic administration, MPTP (Figure 1-1B) readily crosses the bloodbrain barrier and enters glial cells. There, MPTP is converted to 1-methyl-4-phenyl-2, 3dihydropyridium (MPDP+) by monoamine oxidase (MAO)-B. A presumed spontaneous deprotonation within the cyotsol produces the 1-methyl-4-pyridinium ion (MPP+). MPP+ then crosses the glial membrane by a currently unknown mechanism and is actively transported into presynaptic dopaminergic nerve terminals via the plasma membrane dopamine transporter (DAT). MPP+ selectively inhibits complex 1 of the mitochondrial ETC, which then leads to oxidative cell damage and apoptosis (Smeyne \& JacksonLewis 2005). The discovery that environmental compounds, such as MPTP, can cause parkinsonism lead to a search for other potential toxins that may also lead to neuronal cell death (Ballard et al 1985; Langston et al 1983). The isoquinolines (Figure 1-1) are one family of compounds that share remarkable structural homology with MPTP and thus have become strong candidates for a chemical link to dopaminergic cell death in PD (Kajita et al 1994; Nagatsu 1997; Yoshida et al 1993). The tetrahydroisoquinolines (TIQs) and dihydroisoquinolines are members of the isoquinoline family and are potent inhibitors of complex I of the ETC, MAO and a-ketoglutarate dehydrogenase, all known defects in the SN of PD patients (McNaught et al 1995a; McNaught et al 1995b; Thull et al 1995). TIQ and its derivatives (Figure 1-1C) are also natural substances found in 
A<smiles>c1ccc2cnccc2c1</smiles>

Isoquinoline

C<smiles>c1ccc2c(c1)CCNC2</smiles>

TIQ<smiles>CN1CCc2cc(O)c(O)cc2C1</smiles>

$N$-Methyl-Norsalsolinol<smiles>Oc1cc2c(cc1O)CNCC2</smiles>

Norsalsolinol<smiles>CC(C)(C)N1CC=C(c2ccccc2)CC1</smiles>

MPTP<smiles>Oc1cc2c(cc1O)CNCC2</smiles>

(R)Salsolinol<smiles>CN1CCc2cc(O)c(O)cc2C1</smiles>

$N$-Methyl- $(R)$ Salsolinol<smiles>CN1CCc2ccccc2C1</smiles>

N-Methyl-TIQ

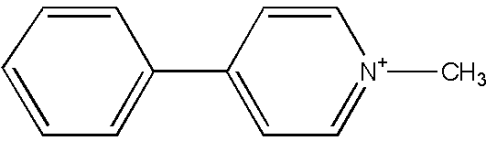

MPP+<smiles>C[C@H]1NCCc2cc(O)c(O)cc21</smiles>

(S)Salsolinol<smiles>C[C@H]1c2cc(O)c(O)cc2CCN1C</smiles>

N-Methyl-(S)Salsolinol<smiles>CC1NCCc2ccccc21</smiles>

1-Methyl-TIQ<smiles>c1ccc(CC2NCCc3ccccc32)cc1</smiles><smiles>COc1ccc(C[C@@H]2c3cc(O)c(OC)cc3CCN2C)cc1O</smiles>

1-Benzyl-TIQ

Reticuline

Figure 1-1. Isoquinoline, MPTP and TIQ derivatives.

(A) Isoquinoline. (B) MPTP is converted to MPP+ by MAO. (C) Chemical structures of selected TIQ derivatives. Reticuline is an exogenous TIQ derivative found in the leaves and bark of Annonaceae trees. 
many plants and have been detected in several common foods such as cheese, bananas, milk, wine and cocoa (Deng et al 1997; Makino et al 1988; Makino et al 1990; Niwa et al 1989). In addition, several TIQ derivatives have also been localized within rodent, PD and normal human brain (Makino et al 1990; Musshoff et al 2003; Musshoff et al 2005; Musshoff et al 2000; Musshoff et al 1999; Niwa et al 1987). Systemic administration of TIQ to marmosets and squirrel monkeys has produced PD-like features, despite the lack of cell death within the SNpc (Nagatsu \& Yoshida 1988; Yoshida et al 1993). Moreover, TIQ appears to be a significantly less potent neurotoxin than MPTP when administered to mice (Ogawa et al 1989). However, there is mounting evidence to suggest that $N$-methylated and benzylated TIQ derivatives (Figure 1-1) are more neurotoxic than their parent compound (Kawai et al 1998; Kotake et al 2003; Kotake et al 1995; Kotake et al 1996; Maruyama et al 1997a; Maruyama et al 1997b; Maruyama et al 1993; Nagatsu 1997; Naoi et al 2000; Naoi et al 1996a; Takahashi et al 1997). Although several TIQ derivatives have been shown to cause cell death in dopaminergic neurons, convincing anatomical proof of cell death in animals exposed to TIQ derivatives, and therefore a link to neurodegenerative disease, is needed.

Central to our hypothesis, TIQ derivatives can be formed endogenously by condensation of dopamine with aldehydes or a-keto acids (Cohen \& Collins 1970; Schöpf \& Bayerle 1934). For example, metabolic pathways may convert neurotransmitters such as dopamine to $N$-methylated TIQ derivatives (Figure 1-2). Cellular processes may also convert exogenously acquired TIQ derivatives into more powerful neurotoxins. TIQ derivatives may thus accumulate in neurons through combinations of endogenous and exogenous means. Susceptible neuronal populations may then gradually shrink in number via apoptotic or necrotic cell death caused by the slow accumulation of these toxins. Unlike the relatively acute effects of MPTP, dopaminergic cell death within the SNpc in idiopathic PD is generally considered to occur gradually over the course of many years. With this in mind, TIQ derivatives are one class of mitochondrial neurotoxins that may exert cytotoxic effects chronically over time and thus be important in the pathogenesis of idiopathic PD.

\subsubsection{Neuronal Production}

Neurons and glia may convert certain TIQs into more toxic derivatives. For example, dopamine may be converted into norsalsolinol or $(R / S)$ salsolinol, which then can be methylated by glial enzymes to form $N$-methyl-norsalsolinol or $N$-methyl$(R / S)$ salsolinol, respectively. Other neurotoxins in this family may be produced endogenously from catecholamines, particularly dopamine, and either aldehydes or $\alpha$ keto acids by several combinations of enzymatic and non-enzymatic pathways.

The synthesis of the $(R / S)$ salsolinol in briefly summarized in Figure 1-2. Endogenous formation of $(R / S)$ salsolinol by Pictet-Spengler condensation of acetaldehyde with dopamine yields a racemic mixture of enantiomers (Cohen \& Collins 1970; Schöpf \& Bayerle 1934). In contrast, stereoselective enzymatic synthesis via salsolinol synthase would generate the $(R)$-enantiomer (Naoi et al 2002; Naoi et al $1996 \mathrm{~b})$. Interestingly, the levels of $(R)$ salsolinol tend to be greater than those of $(S)$ salsolinol in human brain tissue which supports the existence of both mechanisms (Musshoff et al 2003; Musshoff et al 2005; Musshoff et al 2000; Musshoff et al 1999). Norsalsolinol, on the other hand, is generated from the condensation of dopamine with formaldehyde (the Pictet-Spengler condensation). 

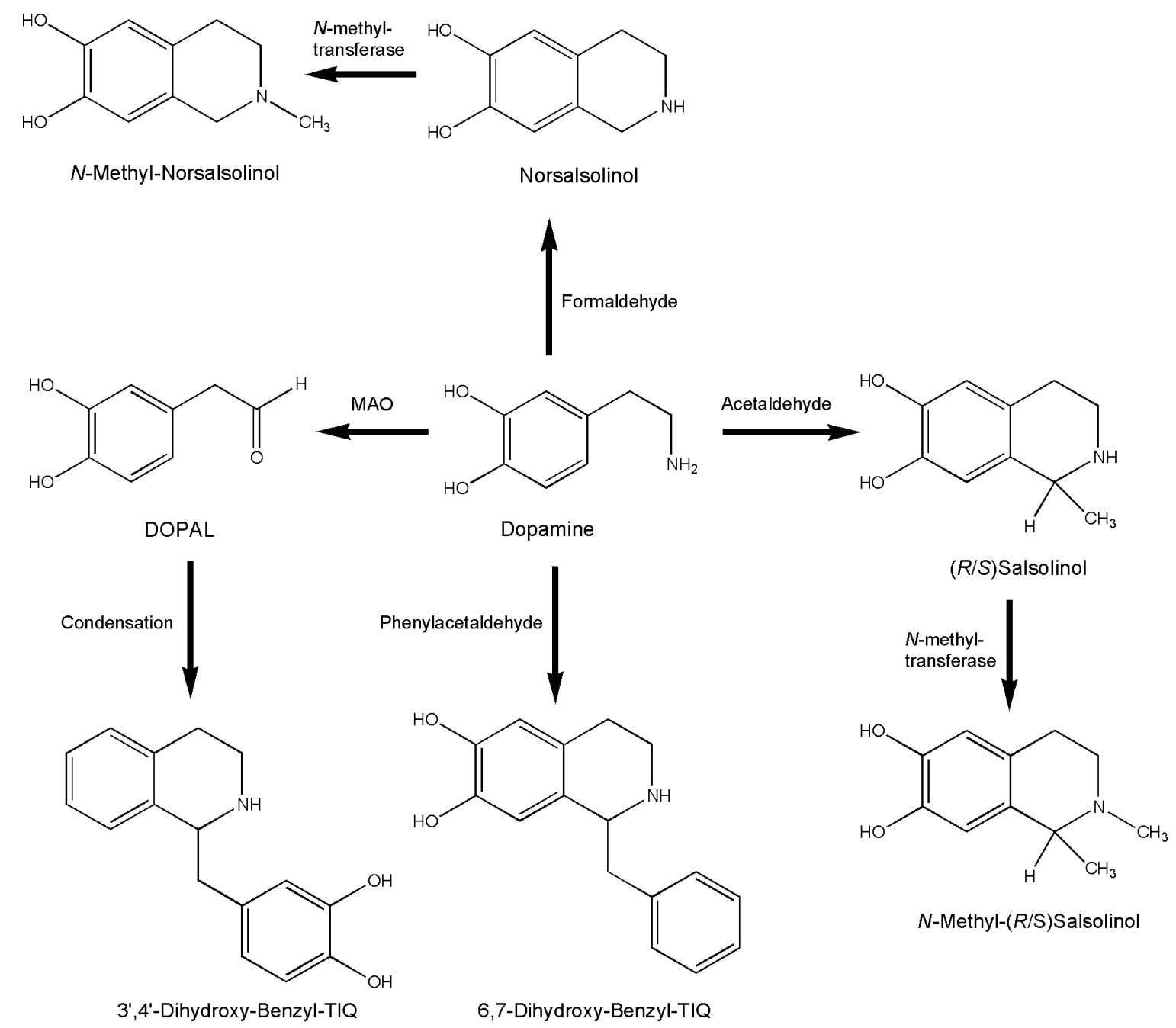

Figure 1-2. Overview of endogenous synthetic pathways.

General schematic for putative endogenous synthesis of $(R / S)$ salsolinol, enantiomeric $N$ methylated salsolinol derivatives and benzyl TIQ derivates from dopamine. 
The methylated derivatives of $(R)$ salsolinol and norsalsolinol appear to be much more toxic than their parent compounds. PD patients have increased $N$-methyltransferase activity that may not be isolated to the brain. Using lymphocytes from peripheral blood, markedly elevated $N$-methyl-transferase activity in PD patients has been observed (Naoi et al 1998b). Several $N$-methyl-transferase enzymes such as phenylethanolamine $N$-methyl-transferase, nicotinamide $N$-methyl-transferase and histamine $N$-methyl-transferase that are present in neurons are capable of TIQ methylation.

Nicotinamide $N$-methyl-transferase (NNMT), a cytosolic enzyme that requires Sadenosyl methionine, catalyzes the catabolic $N$-methylation of nicotinamide to $N$-methylnicotinamide. NNMT protein levels have been measured in the CSF from PD patients and controls (Aoyama et al 2001). The CSF levels of NNMT were higher in younger PD patients than in age-matched controls. NNMT expression was also higher in PD than in control brains using quantitative immunohistochemistry (Parsons et al 2003). Increased NNMT activity could reduce the levels of nicotinamide available for synthesis of nicotinamide adenine dinucleotide and increase the formation of $N$-methylated TIQs (Parsons et al 2003).

Several groups have reported that TIQs can be oxidized to more potent neurotoxins (see next section). Booth and colleagues showed that TIQ and N-methylTIQ are oxidized, but at only 3\% of the rate of MPTP (Booth et al 1989). However, TIQ and many of its derivatives (1-methyl-TIQ, $N$-methyl-TIQ, $(R / S)$ salsolinol, $N$-methyl$(R / S)$ salsolinol, and $N$-methyl-norsalsolinol) do not appear to be substrates for either MAO (Bembenek et al 1990). Instead, isoquinolines and TIQs show selective inhibition of either MAO-A or MAO-B (Bembenek et al 1990; Collins et al 1973; Dostert et al 1989; Meyerson et al 1976; Minami et al 1993; Naoi et al 2004; Thull et al 1995; Yamada 1971). These facts, combined with the lack of physical data proving the successful laboratory synthesis of these ions, make enhanced toxicity of oxidation products unlikely.

However, biosynthetic schemes for benzylated TIQ derivatives depend on precursor compounds generated by MAO. For example, MAO can convert endogenous 2-phenylethylamine to phenylacetaldehyde and dopamine to 3,4dihydroxyphenylacetaldehyde (DOPAL). As shown in Figure 1-2, these compounds can, via condensation, form different benzyl-TIQs: 3',4'-dihydroxy-benzyl-TIQ and 6,7dihydroxy-benzyl-TIQ (Kawai et al 1998).

\subsubsection{Environmental Sources}

TIQs are latent natural substances found in many plants, some of which are consumed by humans and animals as food sources (Rommelspacher \& Susilo 1985). TIQ derivatives have been detected in several common foods and beverages including cheese, cocoa powder, bananas, flour, eggs, beer, milk and even fish such as sardines (Collins et al 1990; Deng et al 1997; Duncan \& Smythe 1982; Duncan et al 1984; Makino et al 1988; Makino et al 1990; Niwa et al 1989; Riggin \& Kissinger 1976; Riggin et al 1976). Various TIQ derivatives are have also been detected in mucuna beans derived from the tropical legume Mucuna pruriens (commonly known as Nescafe, a coffee substitute) (Siddhuraju et al 2000). The commonality of TIQ derivatives in many plantand protein-derived food sources provides an important exogenous link of TIQs to neurodegenerative disease. 
The development of PD has been associated with a number of environmental factors such as pesticides, well water, herbicides, wood pulp and rural living (Barbeau et al 1987; Hertzman et al 1990; Koller et al 1990; Seidler et al 1996; Semchuk et al 1991; 1992; Svenson 1991; Svenson et al 1993; Tanner et al 1989). Rural living and dependence on market gardening might also be related to increased consumption of fresh fruits and vegetables that concentrate TIQ derivatives.

\subsubsection{TIQs and the Blood-Brain Barrier}

For exogenous TIQ derivatives to exert toxic effects on neurons, they must cross the blood-brain-barrier after oral consumption. The blood-brain barrier of mammals is composed of tight junctions between endothelial cells of the central nervous system (CNS) blood vessels in combination with astrocyte foot processes that provide biochemical support. This "barrier" restricts the movement of large or hydrophilic molecules into the cerebrospinal fluid (CSF). Many TIQ derivatives are non-polar molecules with good lipid penetration. In this regard, TIQ and a number of its derivatives have been shown to cross the blood-brain barrier (Kikuchi et al 1991; Makino et al 1988; Thumen et al 2002). Makino and colleagues demonstrated that both 1-methyl-TIQ and TIQ accumulate in rat brain after intraperitoneal injection (Makino et al 1988). Rats were injected with both 1-methyl-TIQ $(5 \mathrm{mg} / \mathrm{kg})$ and TIQ $(5 \mathrm{mg} / \mathrm{kg})$ daily for a period of five days. Two days after the last injection, rats were sacrificed and levels of both compounds were found to be increased over threefold in whole brain homogenate. A study detected TIQ and 1-methyl-TIQ in the brain four hours after oral administration (Kikuchi et al 1991).

However, more hydrophilic TIQ derivatives can also cross the blood-brain barrier. For example, $\mathrm{N}$-methyl-norsalsolinol was present at significantly higher levels than at baseline in the caudate nucleus of rats after intraperitoneal injection (Thumen et al 2002). Interestingly, the $N$-methyl group of $N$-methylated salsolinols may aid blood-brain barrier penetration in some way. Two studies by separate groups were both unable to show transport of salsolinol across the blood-brain barrier after intraperitoneal injection of racemic salsolinol (Origitano et al 1981; Song et al 2006). Since several other research groups have identified and quantified $(R / S)$ salsolinol in brain tissue, its presence may indicate a predominance of endogenous synthesis over exogenous accumulation (Deng et al 1997; Musshoff et al 2003; Musshoff et al 2005; Musshoff et al 2000; Musshoff et al 1999). Transport of other large, hydrophobic TIQ derivatives such as 1-benzyl-TIQ across the blood-brain barrier has also been demonstrated after intraperitoneal injection by both their biochemical and pathological effects on rodent brain and by LC-MS/MS detection (Abe et al 2001; Song et al 2006).

Before reaching the brain, TIQ and its derivatives are metabolized in the liver via first pass metabolism. For example, TIQ accumulation in the rat brain was shown to be enhanced in those with CYP2D6 polymorphisms (poor debrisoquine metabolizers) (Ohta et al 1990). Furthermore, the CYP2D6 enzyme has recently been shown to protect against MPP+ toxicity in SH-SY5Y cells (Mann \& Tyndale 2010). Not surprisingly, several cytochrome P450 polymorphisms have been associated with the development of PD, including several in CYP2D6 (Bon et al 1999; Singh et al 2010). 


\subsubsection{TIQ Membrane Transport}

Several lines of evidence indicate that monoamine transporters could be fundamental to the selective cell death in PD. For instance, the DAT has been shown to be necessary for the in vivo dopaminergic neurotoxicity of MPTP (Gainetdinov et al 1997). In relation to TIQs, Storch and colleagues studied the dopaminergic toxicity of a large selection of isoquinoline derivatives (Storch et al 2002). Human embryonic kidney (HEK-293) and mouse neuroblastoma Neuro-2A cells transfected with the DAT were utilized for cell viability assays. Only $N$-methylated derivatives showed enhanced toxicity in DAT-expressing cell lines. However, the toxicity of $N$-methylated TIQ derivatives was very weak in comparison to MPTP. For example, in HEK-293 cell lines, MPP+ showed almost 1000-fold more DAT-specific toxicity than either $N$-methyl-norsalsolinol or $N$ methyl- $(R / S)$ salsolinol. A recent study by Kotake and colleagues measured the DAT affinity of 17 different isoquinoline derivatives, including isoquinoline, TIQ, 1-methyl-TIQ, $\mathrm{N}$-methyl-TIQ and 1-benzyl-TIQ, in synaptosomes obtained from rat striatum (Kotake et al 2007). A range of 1.7 to $700 \mu \mathrm{M}$ was reported for all compounds. Notably, TIQ exhibited the greatest observed affinity for the DAT. Derivatization of TIQ decreased DAT affinity by at least 100 fold, as seen in 1-methyl-TIQ and 1-benzyl-TIQ.

Unfortunately, salsolinol derivatives were not analyzed.

In mammalian systems, both central and peripheral transport mechanisms exist for the accumulation of TIQs in dopaminergic and noradrenergic neurons.

For instance, in the peripheral nervous system, 6,7-dihydroxy-TIQ is taken up by sympathetic nerve terminals (Cohen et al 1972; Tennyson et al 1973). Uptake is blocked by desmethylimipramine, which indicates that the norepinephrine transporter (NET) is responsible for accumulation of TIQs in sympathetic synapses. Interestingly, 6,7-dihydroxy-TIQ can then accumulate in synaptic vesicles and potentially act as a false neurotransmitter (Tennyson et al 1973).

\subsection{TOXICITY OF TETRAHYDROISOQUINOLINES}

Although neurotoxins may disrupt a variety of cellular activities, cell death precipitated by inhibition of mitochondrial oxidative phosphorylation may be the mechanism most relevant to PD. In a seminal study, the effects of three classes of isoquinoline derivatives (isoquinolines, dihydroisoquinolines and TIQs) and MPP+ were examined on ETC complexes I, II-III and IV from rat brain mitochondria (McNaught et al $1995 b)$. The isoquinoline derivatives or MPP+ were added to reactions mixtures at a concentration of $10 \mathrm{mM}$ and complexes I, II-III and IV activities were compared to controls. MPP+ produced approximately $70 \%$ inhibition of complex I but had no effects on complexes II-III and IV. The effects of TIQ (20\%), N-methyl-TIQ (65\%), $(R / S)$ salsolinol $(55 \%)$ and $N$-methyl-(R/S)salsolinol $(60 \%)$ were less dramatic. Only isoquinoline, 6,7-dimethoxyisoquinoline and $N$-methyl-(R/S)salsolinol produced notable inhibition of complex II-III, and none of the isoquinoline derivatives inhibited complex IV. Notably, there was no significant observed correlation between lipophilicity and complex I inhibition. 


\subsubsection{In Vitro Toxicity}

The in vitro toxicity of TIQ derivatives has been studied using both molecular and morphological assays in a variety of cell lines (Chun et al 2001; Kobayashi et al 2009; Kotake et al 2003; Kotake et al 2007; Maruyama et al 1997b; Naoi et al 2002; Naoi et al 2000; Nijima et al 1991; Nishi et al 1994; Scholz et al 1997; Shavali \& Ebadi 2003; Shavali et al 2003; Storch et al 2000; Wanpen et al 2004). However, the most reliable cell line for studies of TIQ derivative toxicity is arguably the human SH-SY5Y neuroblastoma line. These dopaminergic neurons express the DAT and thus most closely resemble the neurons of the SNpc. Unfortunately, only a handful of studies to date have employed this model. Using these cells, TIQ derivatives have been shown to induce caspase-dependent apoptotic cell death via inhibition of complex I of the ETC with subsequent oxidative DNA damage and inhibition of cellular energy production (Kobayashi et al 2009; Kotake et al 2007; Naoi et al 2002; Shavali \& Ebadi 2003; Storch et al 2000; Wanpen et al 2004).

In general, derivatives of TIQ, namely the members of the salsolinol subfamily, appear to be more toxic than TIQ itself (Kobayashi et al 2009; Kotake et al 2007; Storch et al 2000; Wanpen et al 2004). $N$-methylated salsolinol derivatives appear to be more toxic than their non-methylated parent compound and $(R)$-enantiomers are more toxic than $(S)$-enantiomers. For example, using an assay of DNA fragmentation, $N$-methyl$(R)$ salsolinol was shown to be at least tenfold more toxic than $N$-methyl-( $S$ )salsolinol (Maruyama et al 1997b). Additionally, apoptosis has been demonstrated with TUNEL labeling, Hoechst 33342 staining and Western blot detection of caspase- 3 activation (Akao et al 1999; Maruyama et al 1997b). SH-SY5Y overexpressing Bcl-2 (an antiapoptotic mitochondrial membrane protein) are resistant to apoptosis by $(R)$ salsolinol enantiomers (Maruyama et al 2001). Interestingly, methylation at the N-position has also been shown to decrease a compound's affinity for the DAT (Kotake et al 2007) However, this study failed to demonstrate a significant relationship between cytotoxicity and affinity for the DAT, and uptake of most the TIQ derivatives seemed to be dependent on lipophilicity rather than active transport.

The most toxic TIQ derivatives, however, appear to be the benzyl-TIQs (Kotake et al 2003; Kotake et al 2007; Shavali \& Ebadi 2003). In SH-SY5Y cells, the IC 50 values of several benzylated TIQs ranged from 0.02 to $0.09 \mathrm{mM}$ after $48 \mathrm{hrs}$ of exposure, several orders of magnitude lower than TIQ or N-methylated TIQ derivatives (Kotake et al 2007). Furthermore, these compounds are very lipophilic and pass through the bloodbrain barrier readily (Song et al 2006). However, it should be noted that to date only 1benzyl-TIQ has been detected in either rodent or human brain, and plant levels of 1benzyl-TIQ are extremely low in comparison to other TIQ and salsolinol derivatives. Thus, while the benzyl-TIQs may be more toxic, rarity in the brain and foods may limit their relevance to PD pathogenesis.

Much like MPTP, at lower doses several TIQ derivatives have been shown to inhibit tyrosine hydroxylase (TH) (Hirata \& Nagatsu 1985; Maruyama et al 1993; Scholz et al 1997). In a comparison of six $N$-methylated salsolinol derivatives, $N$-methylnorsalsolinol was found to be the most potent inhibitor of TH (Maruyama et al 1993). A separate report demonstrated that the inhibition of $\mathrm{TH}$ by $\mathrm{N}$-methyl-norsalsolinol was noncompetitive with an $\mathrm{IC}_{50}$ of $10 \mu \mathrm{M}$ (Scholz et al 1997). Therefore, reductions in dopamine content and $\mathrm{TH}$ immunoreactivity noted in many reports may be manifestations of TH inhibition. However, whether neuronal TH inhibition is a direct 
effect TIQs on the enzyme or their overall reduction in cellular energy production remains to be seen.

\subsubsection{In Vivo Toxicity}

To date, in vivo neurotoxicological studies of TIQ and its derivatives have failed to provide convincing evidence of cell death within the SNpc. Studies in marmosets and squirrel monkeys suggest that both the behavioral and biochemical effects of subcutaneously injected TIQ in doses up to $20 \mathrm{mg} / \mathrm{kg} /$ day for 100 days could be due to TH inhibition rather than cell death within the SNpc (Nagatsu \& Yoshida 1988; Yoshida et al 1993). These primates exhibited rigidity and bradykinesia, reduced nigral dopamine and decreased TH activity. TIQ was injected subcutaneously on a daily basis for at least four weeks in two studies utilizing C57BL/6J mice (Ogawa et al 1989; Perry et al 1988). Reduced TH immunoreactivity in the absence of overt cell death was again reported. Surprisingly, there was no reduction in striatal dopamine, 3,4-

dihydroxyphenylacetic acid (DOPAC) or homovanillic acid (HVA) content.

In a single report of $N$-methyl-TIQ administration in marmosets, three out of four animals died after prolonged subcutaneous injection (Perry et al 1987). Unfortunately, pathological findings were not reported and the single surviving marmoset showed no reduction in dopamine, DOPAC or HVA content. Rhesus monkeys exposed to prolonged administration of $N$-methyl-TIQ again showed no reduction in dopamine, DOPAC or HVA content and only slight motor disturbance. In the same report, cynomolgous monkeys exposed to $N$-methyl-isoquinolinium ion exhibited only mild bradykinesia after eight weeks of intraventricular administration (Yoshida et al 1993). However, in mice, intraperitoneal injection of $N$-methyl-TIQ for 20 weeks was associated with atrophy of the SNpc and reduced nigral TH immunoreactivity (Fukuda 1994). In rats, $\mathrm{N}$-methyl-isoquinolinium ion reportedly demonstrated cytotoxicity when directly infused into the striatum (Booth et al 1989).

Naoi and coworkers delivered $N$-methyl-(R)salsolinol to rats by intrastriatal infusion with a mini-osmotic pump over a period of one week (Naoi et al 1996a). Parkinsonian behavioral changes, including hypokinesia, stiff tail and postural abnormalities were noted in treated rats. Dopamine and norepinephrine levels were reduced in both the striatum and $\mathrm{SN}$ and $\mathrm{TH}$-immunoreactive neurons were reduced by more than $50 \%$ in the striatum of infused animals. Neuronal density was also reduced, although the differences between control and injected sides did not reach statistical significance. $\mathrm{N}$-methyl-norsalsolinol was injected into the left medial forebrain bundle of rats and both the behavioral response to apomorphine and striatal dopamine levels were determined three weeks later (Moser et al 1996b). There was no response to apomorphine, and the mild reductions in dopamine, DOPAC and HVA did not reach statistical significance.

In a single monkey, chronic subcutaneous injection of 1-benzyl-TIQ produced behavioral parkinsonism (Kotake et al 1996). Morphological and biochemical effects were not reported. After short-term intraperitoneal injections of 1-benzyl-TIQ in the mouse, motor testing demonstrated mild parkinsonism that was corrected by carbidopa/levodopa (Kotake et al 1995). In another study, mice receiving 1-benzyl-TIQ intraperitoneally were bradykinetic, but showed no reductions in striatal dopamine levels or TH+ neurons in the SNpc (Abe et al 2001). In yet another report, intraperitoneal 
injections of 1-benzyl-TIQ were performed for 17 days in mice prior to quantification of striatal catecholamines (Antkiewicz-Michaluk et al 2001). Chronic injection of 1-benzylTIQ was associated with muscular rigidity and a $60 \%$ reduction in striatal dopamine levels. The benzyl-TIQ derivative 3',4'-dihydroxy-benzyl-TIQ induced behavioral parkinsonism in mice after five days of intraperitoneal administration, but striatal dopamine levels and SNpc morphology were not reported (Kawai et al 1998). In the same report, administration of 1-benzyl-6,7-dihydroxy-TIQ produced no behavioral effects.

Unfortunately, the above in vivo studies are largely invalid, riddled with poor to non-existent synthetic mechanisms of TIQ derivatives and antiquated delivery systems. For instance, all studies utilizing oxidized TIQ derivatives (e.g. $N$-methyl-isoquinolinium ion) were reportedly produced by the action of purified MAO enzyme. Besides the fact that several studies report that TIQs are not substrates for or even inhibit MAO, the chances of successfully isolating purified ions for administration from a protein mixture are very poor. With this in mind, no study to date has published any physical data to prove successful synthesis of isoquinolinium ions. Furthermore, peripheral administrations of even supraphysiologic doses of TIQ derivatives are unlikely to produce toxic levels within the critical dopaminergic neurons of the SNpc when enzymes such as CYP2D6 and likely other metabolic mechanisms are taken into account. Finally, as demonstrated in several reports, the cytotoxicities of TIQs are orders of magnitude weaker than MPTP or even rotenone. With this in mind, TIQ derivative delivery may require months or even years to produce convincing evidence of cell death. Without question, more data describing the exact mechanism(s) of cellular penetration and toxicity of TIQ derivatives in the in vitro model is necessary for proper in vivo experimental design.

\subsection{TETRAHYDROISOQUINOLINES IN PARKINSON DISEASE}

The brain and CSF concentrations of several TIQ derivatives have been determined in humans with PD (Antkiewicz-Michaluk et al 1997; Kotake et al 1995; Maruyama et al 1999; Moser et al 1995; Muller et al 1999; Niwa et al 1987; Niwa et al 1991; Sandler et al 1973). In a single PD case, TIQ was quantified in the frontal cortex at $10 \mathrm{ng} / \mathrm{g}$ (Niwa et al 1987). Analysis of frontal cortex from a single normal human brain detected TIQ at less than $1 \mathrm{ng} / \mathrm{g}$. This same study also quantified $N$-methyl-TIQ in frontal cortex at $2 \mathrm{ng} / \mathrm{g}$ in PD brain and $3 \mathrm{ng} / \mathrm{g}$ in control brain, whereas 1-methyl-TIQ was reportedly undetectable in both normal human and PD brain. In a subsequent publication from the same group, TIQ was detected in the occipital cortex of a single PD brain at $7 \mathrm{ng} / \mathrm{g}$ (Niwa et al 1991). The same study only provided "estimated" wet tissue concentrations for both $N$-methyl-norsalsolinol and $N$-methyl- $(R / S)$ salsolinol. The use of single brains and estimated concentrations provide little meaningful information and therefore no reasonable conclusions can be made. A more rigorous and systematic topological analysis of TIQ derivatives in PD and normal human brain is obviously needed.

$(R / S)$ salsolinol has been detected in the plasma and urine of both PD patients and controls. Concentrations of $(R / S)$ salsolinol were higher in plasma than in CSF in one study (Muller et al 1999). Although the differences did not reach statistical significance, both salsolinol enantiomers were detected at lower concentrations in PD patients than in controls. The CSF levels of $N$-methyl- $(R)$ salsolinol levels were reported 
to decrease after two years of treatment with levodopa (Maruyama et al 1999). In another study, $\mathrm{N}$-methyl-norsalsolinol was detected in the CSF of PD patients and negatively correlated with disease duration (Moser et al 1995). Interestingly, $N$-methylnorsalsolinol was not detected in the CSF of controls. This data may suggest that, over time, dying dopaminergic neurons release $(R / S)$ salsolinol, $N$-methyl-norsalsolinol and $N$ methyl-(R/S)salsolinol into the CSF.

The risk of PD has been shown to be decreased by certain behaviors such as smoking cigarettes and drinking coffee (Grandinetti et al 1994; Paganini-Hill 2001). Interestingly, some components in tobacco smoke limit the conversion of TIQ to more toxic $N$-methylated derivatives (Soto-Otero et al 1996). Administration of cigarette smoke has also been shown to reduce the brain tissue levels of TIQ in rats (Soto-Otero et al 2001). A logical hypothesis derived from this data could suggest that decreased accumulation and/or metabolism of TIQ in the brain of smokers explains the epidemiological effect of cigarette smoking on the incidence of PD.

Finally, a role for neurotoxins in the pathogenesis of idiopathic PD may also be suggested by the incidence of levodopa-resistant atypical parkinsonism on the French West Indies island of Guadeloupe. This syndrome is similar in nature to an earlier discovery in Guam and may be caused by exposure to tropical plants containing mitochondrial complex I inhibitors (quinolines, acetogenins and rotenoids) found in certain fruit and teas made from trees in the Annonaceae family (Caparros-Lefebvre et al 2002). These tree products contain known neurotoxins like reticuline (Figure 1-1), a benzyl-TIQ derivative. Patients with Guadeloupean Parkinsonism present with rigidity and bradykinesia, in addition to ophthalmoparesis, pseudo-bulbar palsy and frontal lobe signs similar to those of progressive supranuclear plasy (PSP). In a study by Lannuzel and co-workers, the $\mathrm{IC}_{50}$ for reticuline in cultured mesencephalic dopaminergic neurons at $24 \mathrm{hrs}$ was $100 \mu \mathrm{M}$ (Lannuzel et al 2002). Furthermore, acetogenins like annonacin appear to be more potent inhibitors of complex I than most TIQ derivatives (Lannuzel et al 2003; Yuan et al 2003). When added to mesencephalic cultures for $24 \mathrm{hrs,}$ annonacin was much more potent than $\mathrm{MPP}+\left(\mathrm{IC}_{50}=0.018 \mu \mathrm{M}\right.$ vs.1.9 $\left.\mu \mathrm{M}\right)$ in killing dopaminergic neurons and overall dopamine uptake was reduced. Toxic effects were even seen at very low concentrations when the incubation time was extended by several days.

\subsection{SIGNIFICANCE OF STUDY}

The risk of developing idiopathic PD is due to the effects and interactions of numerous genetic and environmental risk factors. Subtle environmental risk factors could be those associated with the consumption or ingestion of undefined neurotoxins through the respiratory and gastrointestinal systems and accumulation in the brain over a lifetime. Despite recent advances in genetics and epidemiology, the etiopathogenesis of sporadic PD remains obscure in the vast majority of cases.

In PD, the most prominent neurodegenerative cell death is seen in the dopaminergic neurons of the SNpc and noradrenergic neurons of the locus ceruleus. Thus a straightforward explanation for the relatively selective cell death seen in PD is the accumulation of neurotoxins derived from catecholamines. Isoquinoline derivatives (isoquinolines, dihydroisoquinolines and TIQs) have been shown to inhibit complex I of the mitochondrial ETC and are one family of compounds that can be synthesized from 
catecholamine precursors. Several TIQs can cross the blood-brain barrier and certain TIQs are taken up by the NET. TIQ derivatives may thus accumulate in dopaminergic neurons over time and, upon reaching critical levels, could generate oxidative stress and initiate apoptosis by inhibiting complex I of the mitochondrial ETC. Dopaminergic neurons in elderly persons with a naturally lowered oxidative phosphorylation capacity may not tolerate a significant buildup of complex I inhibitors and in time these cells may die.

Although considerable evidence suggests that isoquinolines such as the TIQs may contribute to dopaminergic cell loss in PD, data detailing the anatomical distribution and definitive toxicity of these potential neurotoxins in mammalian tissues is unfortunately inconsistent and unreliable. Using enantiomeric-selective HPLC-EC and LC-MS/MS, the concentrations of TIQ, 1-methyl-TIQ, N-methyl-TIQ, 1-benzyl-TIQ, $(R)$ salsolinol, $(S)$ salsolinol, $N$-methyl- $(R)$ salsolinol, $N$-methyl-( $S)$ salsolinol, norsalsolinol and $N$-methyl-norsalsolinol were systematically determined in multiple regions of rodent, human, and PD brain (DeCuypere et al 2008a; DeCuypere et al 2008b). As TIQ derivates have been demonstrated to be normal constituents of many plants, these compounds were quantified in the 20 most commonly consumed fruits and vegetables in the United States. Furthermore, data detailed in Chapter 4 demonstrates the definitive neurotoxicity of $\mathrm{N}$-methylated derivatives of salsolinol in dopaminergic SH-SY5Y cells. Based on our results, it is logical to conclude that several tetrahydoisoquinoline toxins (such as $N$-methyl-(R/S)salsolinol and $N$-methyl-norsalsolinol), all of which inhibit complex I of the ETC, can accumulate in neurons with aging and contribute to neurodegeneration via apoptotic cell death. Prevention of the onset and progression of PD demands a profound and comprehensive understanding of potential neurotoxins and their relationship to disease pathogenesis. 


\section{CHAPTER 2. LOCALIZATION OF N-METHYL-NORSALSOLINOL WITHIN RODENT AND HUMAN BRAIN ${ }^{*}$}

\section{$2.1 \quad$ INTRODUCTION}

The discovery that MPTP can cause Parkinsonism (Ballard et al 1985; Langston et al 1983) led to a search for MPTP analogues as possible endogenous or exogenous dopaminergic neurotoxins. As shown in Figure 2-1, $N$-methylated isoquinolines share structural homology with MPTP. Currently available data suggests that isoquinoline derivatives may be endogenous and/or exogenous neurotoxins that contribute to dopaminergic cell death in PD. For example, McNaught and colleagues showed that several isoquinoline derivatives are potentially more potent inhibitors of complex I of the respiratory chain than the MPTP derivative, MPP+ (McNaught et al 1995b).

Both "exogenous" and "endogenous" pathways have been proposed for isoquinoline neurotoxicity. In the exogenous pathway depicted in Figure 2-1B, TIQ is acquired from dietary sources, transported across the blood-brain barrier, converted by an $N$-methyl-transferase to $N$-methyl-TIQ, and then oxidized to $N$-methylisoquinolium ion (NMIQ ${ }^{+}$) by a MAO (Makino et al 1988; Niwa et al 1989). Nagatsu and Yoshida stirred interest in TIQ and related compounds by showing that systemic administration of TIQ produced Parkinsonism in marmosets despite the absence of demonstrable cell death within the substantia nigra pars compacta (Nagatsu \& Yoshida 1988). Similarly, TIQ does not appear to produce dramatic dopaminergic cell death in mice (Ogawa et al 1989). However, cell death could have easily been missed in both studies since neither markers of apoptosis nor stereological counting methods were employed. Irrespective of these caveats, $N$-methylated derivatives of TIQ appear to be more neurotoxic than TIQ and have been shown to destroy cultured dopaminergic neurons (Nijima et al 1991; Nishi et al 1994).

As shown in Figure 2-1C, $\mathrm{N}$-methylated isoquinoline derivatives such as $\mathrm{N}$ methyl-6,7-dihydroxy-1,2,3,4-tetrahydroisoquinoline ( $N$-methyl-norsalsolinol) can be formed by a special type of condensation from catecholamines and aldehydes (Cohen \& Collins 1970) in the "endogenous" pathway of isoquinoline neurotoxicity. Furthermore, $\mathrm{N}$-methyl-norsalsolinol has been identified in normal human and PD brain using gas chromatography-mass spectrometry (Niwa et al 1991; Niwa et al 1993). Nmethyl-norsalsolinol has also been detected in the CSF of PD patients but not in normal human controls (Moser et al 1995). N-methyl-norsalsolinol inhibits both $\mathrm{TH}$ (Scholz et al 1997) and MAO activity (Moser et al 1996a) and may be neurotoxic (Maruyama et al 1993; Naoi et al 1995).

To address the issue of neuronal accumulation and the subsequent potential for neurotoxicity, the cellular and cell-type localization and regional distribution of $N$ methylated isoquinoline derivatives must first be determined. In this study, highperformance liquid chromatography with electrochemical detection (HPLC-EC) was used to quantify the regional distribution of $\mathrm{N}$-methyl-norsalsolinol in mouse, rat, and human brain. Due to the limited spatial resolution of liquid chromatography, a monoclonal

\footnotetext{
*Permission to reproduce from Wiley-Liss, Inc. DeCuypere M, Kalabokis VN, Hao R, Schroeder D, Miller DD, LeDoux MS. 2008. Localization of N-methyl-norsalsolinol within rodent and human brain. J Neurosci Res 86:2543-52.
} 

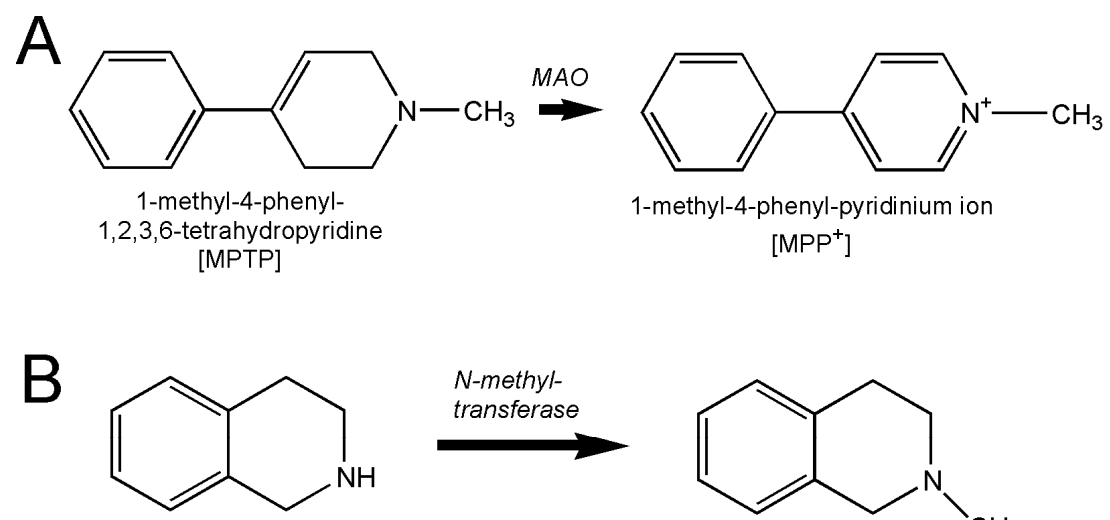<smiles>CN1CCc2ccccc2C1</smiles>

tetrahydroisoquinoline [TIQ]

$\mathrm{N}$-methyl-tetrahydroisoquinoline [NMTIQ]<smiles></smiles>

$\mathrm{N}$-methylisoquinolinium ion [NMIQ ${ }^{+}$]

C<smiles>NCCc1ccc(O)c(O)c1</smiles><smiles>Oc1cc2c(cc1O)CNCC2</smiles><smiles>C[n+]1ccc2cc(O)c(O)cc2c1</smiles>

$\mathrm{N}$-methyl-6,7-dihydroxy-isoquinolinium ion<smiles>CN1CCc2cc(O)c(O)cc2C1</smiles>

Figure 2-1. Synthesis of MPP+ and isoquinoline neurotoxins.

(A) MPTP crosses the blood-brain barrier and is converted to the neurotoxin MPP ${ }^{+}$by a monoamine oxidase (MAO). (B) One potential exogenous pathway for tetrahydroisoquinoline (TIQ) neurotoxicity. (C) One potential endogenous pathway for synthesis of $\mathrm{N}$-methyl-norsalsolinol and its neurotoxic derivative $N$-methyl-6,7-dihydroxyisoquinolinium ion. 
antibody to $N$-methyl-norsalsolinol was generated and used to characterize the cellular and cell-type localization of this $\mathrm{N}$-methylated isoquinoline. PD brain samples were also analyzed to help address previously reported findings that (1) $N$-methyl-norsalsolinol cannot be detected in normal human CSF by liquid chromatography and (2) CSF levels of $\mathrm{N}$-methyl-norsalsolinol are negatively correlated with disease duration in PD (Moser \& Kompf 1992; Moser et al 1995).

\subsection{MATERIALS AND METHODS}

All experiments were approved by the University of Tennessee Health Science Center Animal Care and Use Committee and performed in accordance with the National Institutes of Health Guide for the Care and Use of Laboratory Animals. Mice and rats were maintained in a temperature-controlled environment with free access to food and water.

\subsubsection{Chemicals}

Acetaldehyde, dimethylsulfate, ethylenediaminetetraacetic acid (EDTA), formaldehyde, ortho-phosphoric acid, papaveroline, perchloric acid, serotonin hydrochloride, sodium bisulfite, sodium cyanoborohydride, tetrahydroisoquinoline, triethylamine (TEA), 3-hydroxytyramine, 3,4-dihydroxybenzylamine (DHBA), 9Hpyrido[3,4-b]indole, and 1,2,3,4-tetrahydro-9H-pyrido[3,4-b]indole were purchased from Sigma-Aldrich (St. Louis, MO, USA) and were used without further treatment. 5hydroxyisoquinoline was purchased from Sigma-Aldrich and was recrystallized twice from methanol. Sulfosuccinimidyl-6-(biotinamido) hexanoate was purchased from Pierce (Rockford, IL, USA). Sodium octylsulphonate (SOS) and monobasic anhydrous sodium dihydrogen phosphate used in mobile phase preparation were purchased from Fluka Chemie (Buchs, Switzerland). HPLC grade water, acetonitrile, and $\beta$ cyclodextrin were obtained from Fisher Scientific (Hampton, NH, USA).

\subsubsection{Synthesis of Derivatives of TIQ and Isoquinoline}

An aqueous solution of 3-hydroxytyramine $(0.5 \mathrm{M})$ was mixed with a four-fold molar excess of formaldehyde to prepare 6,7-dihydroxy-1,2,3,4-tetrahydroisoquinoline (norsalsolinol) or a four-fold molar excess of acetaldehyde to prepare $(R / S)$ salsolinol. The $\mathrm{pH}$ of the solution was adjusted to 4.5 and the sample was incubated overnight at room temperature under nitrogen in the dark. The solvent was evaporated under diminished pressure and the product was crystallized from isopropyl alcohol.

The tertiary amines $N$-methyl-norsalsolinol and $N$-methyl-(R/S)salsolinol were prepared from the respective secondary amines norsalsolinol and $(R / S)$ salsolinol by reductive methylation in the presence of formaldehyde and sodium cyanoborohydride. The reaction mixture contained $0.5 \mathrm{M}$ of the secondary amine, $2.5 \mathrm{M}$ formaldehyde, 0.2 $\mathrm{M}$ sodium acetate, and $1 \mathrm{M}$ sodium cyanoborohydride at $\mathrm{pH} 5$. The reaction mixture was incubated at room temperature overnight under nitrogen in the dark. The sample was clarified by centrifugation and the clear solution was acidified with $\mathrm{HCl}$ to $\mathrm{pH} 0.1$ to decompose unreacted cyanoborohydride. The solvent was evaporated and the product was extracted from the residue with isopropyl alcohol. Evaporation of the 
solvent and extraction of the residue was repeated twice. The final product was stored as a solution in isopropyl alcohol at $-20^{\circ} \mathrm{C}$.

$\mathrm{N}$-methyl-5-hydroxyisoquinoline was prepared according to Schulman and Fernando (Schulman \& Fernando 1968). Dimethylsulfate was used as the methylating agent. 5-hydroxyisoquinoline was dissolved in anhydrous ethanol and allowed to react with a two-fold molar excess of dimethylsulfate for $20 \mathrm{hrs}$. The excess of dimethylsulfate was destroyed by the addition of water. The solvent was evaporated and the product was crystallized from methanol. The structures of all synthesized compounds were confirmed by mass spectrometry.

\subsubsection{HPLC-EC Analysis of N-Methyl-Norsalsolinol}

\subsubsection{Chromatography Standard and Sample Preparation}

Primary stock standard solutions were prepared by dissolving $10 \mathrm{mg}$ of $\mathrm{N}$-methylnorsalsolinol in $25 \mathrm{ml}$ of a dissolution buffer, which consisted of $0.1 \mathrm{M}$ perchloric acid, $0.1 \mathrm{mM}$ sodium bisulfite, and $0.1 \mathrm{mM}$ EDTA. These concentrates were then were then divided into $1 \mathrm{ml}$ aliquots, frozen, stored at $-80^{\circ} \mathrm{C}$, and thawed prior to use at $4{ }^{\circ} \mathrm{C}$. Working standards in the $\mathrm{nM}$ range were freshly prepared in dissolution buffer prior to each assay. Standard curves employed for analyte quantitation were generated with the internal standard 3,4-dihydroxybenzylamine (DHBA). The relationship between concentration and relative response was linear over two orders of magnitude (sample correlation coefficient $>0.98$ ), with an overall electrochemical sensitivity of $0.001 \mathrm{nA}$. The detection limit for $\mathrm{N}$-methyl-norsalsolinol was $20 \rho \mathrm{g}$ per injection in standards and $28 \rho g$ per injection in tissue.

Mice $(N=5$, age $=3.5 \mathrm{mo}$, Balb/c strain, Jackson Labs, Bar Harbor, Maine, USA) and rats $(N=5$, age $=3.5 \mathrm{mo}$, Sprague-Dawley strain, Harlan Sprague Dawley, Inc., Indianapolis, IN, USA) were anesthetized with aerosolized halothane and fresh brain samples were obtained and partitioned using a dissecting microscope. Tissue samples $(0.1-0.2 \mathrm{~g})$ of striatum, ventral midbrain, frontal cerebral cortex, cerebellar cortex, and hippocampus were weighed and then homogenized in $100 \mu \mathrm{l}$ of ice-cold dissolution buffer per $10 \mathrm{mg}$ wet weight. Cryopreserved blocks of fresh frozen post-mortem brain tissue from normal human controls $(N=5$, mean age $=70.4$ years, standard deviation $=$ 9.4 years $)$ and human patients with a clinical diagnosis of PD $(N=5$, mean age $=69.6$ years, standard deviation $=10.8$ years) used for HPLC-EC analysis were obtained from the National Institute of Child Health and Human Development (NICHD) Brain and Tissue Bank for Developmental Disorders under contracts N01-HD-4-3368 and N01-HD4-3383 (Baltimore, MD, USA). Frozen blocks of caudate nucleus, putamen, substantia nigra, frontal cerebral cortex, cerebellar cortex, locus ceruleus, and hippocampus were partitioned into $0.2-0.4 \mathrm{~g}$ fragments and homogenized as above. All homogenates were centrifuged at $20,000 \mathrm{~g}$ for $25 \mathrm{~min}$ at $4^{\circ} \mathrm{C}$. Supernatants were filtered through $0.22 \mu \mathrm{m}$ pore size polyvinylidene fluoride (PVDF) syringe-driven membrane filters (Millipore Corp., Bedford, MA, USA) and immediately frozen and stored at $-80{ }^{\circ} \mathrm{C}$ until the time of analysis. The recovery of $N$-methyl-norsalsolinol was $87.6+/-7.2 \%$ based on spiked (2.0 ng standard) tissue samples subjected to the above extraction procedure. 


\subsubsection{Mobile Phase Preparation}

A stock buffer solution containing $75 \mathrm{mM}$ monobasic sodium dihydrogen phosphate, $2 \mathrm{mM}$ SOS, $25 \mu \mathrm{M}$ EDTA, $20 \mathrm{mM} \beta$-cyclodextrin, and $100 \mu \mathrm{l}$ of TEA was prepared in $1800 \mathrm{ml}$ of HPLC grade water. To prepare the mobile phase, this solution was then mixed with $200 \mathrm{ml}$ of HPLC grade acetonitrile and buffered to $\mathrm{pH} 3.0$ using concentrated ortho-phosphoric acid. The mobile phase was stored at room temperature for no more than 2 days, filtered through a $0.20 \mu \mathrm{m}$ pore size white nylon filter membrane (Millipore Corp.), and degassed under vacuum for 30 min prior to use.

\subsubsection{Liquid Chromatography}

HPLC-EC analysis was performed with an ESA Model 5600A CoulArray ${ }^{\circledR}$ system (ESA Inc., Chemlsford, MA, USA), equipped with Shimadzu Model DGU-14A on-line degassing unit (Shimadzu Scientific Instruments, Columbia, MD, USA), an ESA Model 582 pump, and an ESA Model 542 refrigerated autosampler. The detection system consisted of three coulometric array modules, each containing four electrochemical detector cells. Electrode potentials were selected over the range of 0 to $+700 \mathrm{mV}$, with a $50 \mathrm{mV}$ increment against palladium electrodes. Chromatographic separation was achieved by auto-injecting $30 \mu \mathrm{l}$ sample aliquots at $5^{\circ} \mathrm{C}$ onto a MetaChem Intersil (MetaChem Technologies, Torrance, CA, USA) reversed-phase $\mathrm{C}_{18}$ column (5 $\mu \mathrm{m}$ particle size, $250 X 4.6 \mathrm{~mm}$ I.D) with an ESA Hypersil $\mathrm{C}_{18}$ pre-column (5 $\mu \mathrm{m}$ particle size, 7.5X4.6 mm I.D.). A mobile phase flow rate of $1.25 \mathrm{ml} / \mathrm{min}$ and analysis time of $45 \mathrm{~min}$ was used for all experiments. System control and data acquisition/processing were performed using ESA CoulArray software (version 1.02). All samples were processed in technical triplicate with median values used for mean calculations. The results of HPLCEC experiments were analyzed by means of a student's $t$ test for each region of interest. An alpha $(\alpha)$ of 0.05 was chosen for statistical significance.

\subsubsection{Synthesis of Immunogen}

Cationized bovine serum albumin (cBSA) was used as a carrier protein for immunizations. cBSA was prepared by coupling the carboxylates of BSA to ethylene diamine in the presence of 1-ethyl-3-(3-dimethylaminopropyl)-carbodiimide (Hermanson 1996). N-methyl-norsalsolinol was cross-linked to cBSA using the Mannich reaction. In this procedure the carbon atoms at ortho-positions to the phenolic group of the hapten are attached to the primary amino groups of the protein through a methylene group. Haptens were similarly attached to cationized lysozyme to facilitate the screening of monoclonal antibodies.

\subsubsection{Production of Monoclonal Antibody}

The antibody was produced by a hybridoma obtained from a mouse that had been immunized with $N$-methyl-norsalsolinol coupled to cBSA. The IgG fraction from ascites fluid was purified by Protein $G$ chromatography. Initial screenings were done against a panel of six different compounds by direct enzyme-linked immunoabsorbant 
assay (ELISA). Positive clones were subcloned and screened against the specific compound.

\subsubsection{Antibody Characterization}

\subsubsection{Tracer Synthesis}

The tracer was $\mathrm{N}$-methyl-norsalsolinol labeled with biotin. Biotinylation of $\mathrm{N}$ methyl-norsalsolinol was carried out in two steps. A linker $(\mathrm{H} 2 \mathrm{NCH} 2 \mathrm{CH} 2 \mathrm{NH} 2)$ containing a primary amino group was introduced at carbon atom 5 or 8 of $\mathrm{N}$-methylnorsalsolinol. This modification occurred by treating $N$-methyl-norsalsolinol with a 20fold molar excess of ethylene diamine and 50-fold molar excess of formaldehyde in 0.1 $\mathrm{M}$ MES buffer, $\mathrm{pH} 4.8$, at $50{ }^{\circ} \mathrm{C}$ for $24 \mathrm{hrs}$. Excess of unreacted ethylene diamine was removed by precipitation with ten volumes of methanol. The methanol precipitation step was repeated twice following evaporation of the solvent. The primary amino group of the modified $\mathrm{N}$-methyl-norsalsolinol was labeled with biotin in a reaction with a three-fold molar excess of sulfosuccinimidyl-6-(biotinamido) hexanoate in $50 \mathrm{mM}$ phosphate, $\mathrm{pH} 8$.

\subsubsection{Inhibition Assay}

The cross-reactivities of the antibody with structurally related compounds to $N$ methyl-norsalsolinol were estimated by competitive inhibition assay. The assay buffer for all the measurements was Tris-buffered saline, $\mathrm{pH} 7.5$, containing $1 \mathrm{mg} / \mathrm{ml} \mathrm{BSA}$ and $0.05 \%$ Tween 20 . All assays were carried out at room temperature. Cross reactivities were determined by competitive inhibition experiments where a trace amount of biotinylated $\mathrm{N}$-methyl-norsalsolinol competed with various inhibitors for binding to the antibody. The relative amount of antibody- $N$-methyl-norsalsolinol-biotin complex was determined by HRP-labeled streptavidin (R\&D Systems, Inc., Minneapolis, MN, USA). The apparent dissociation constants of the interaction of the antibody with $\mathrm{N}$-methylnorsalsolinol and its biotinylated analogue were 0.6 and $2 \mu \mathrm{M}$, respectively.

In a typical experiment the antibody was coated on the wells of microtiter plates at $2 \mu \mathrm{g} / \mathrm{ml}$ in PBS for $24 \mathrm{~h}$ at $4{ }^{\circ} \mathrm{C}$. The wells were blocked with a solution composed of assay buffer supplemented with $5 \mathrm{mg} / \mathrm{ml}$ of BSA and were rinsed twice with assay buffer prior to use. Aliquots of $100 \mu \mathrm{l}$ of solutions containing a fixed concentration of tracer $(20 \mathrm{nM})$ and various concentrations of competitors were added to the wells and the samples were incubated for $2.5 \mathrm{hrs}$. The wells were rinsed three times with assay buffer and $100 \mu \mathrm{l}$ of HRP-streptavidin conjugate was added to each well. Following a 1 $\mathrm{hr}$ incubation, the wells were rinsed five times with assay buffer and $100 \mu \mathrm{l}$ of color development reagent (R\&D Systems) was added. The reaction was stopped by the addition of $100 \mu \mathrm{l}$ of $1 \mathrm{M} \mathrm{H}_{2} \mathrm{SO}_{4}$ and the color was read at $450 \mathrm{~nm}$ in a plate reader.

\subsubsection{Absorption Control}

$\mathrm{N}$-methyl-norsalsolinol was mixed with the monoclonal antibody to $\mathrm{N}$-methylnorsalsolinol or with an antibody to TH in a proportion of 10:1 (antigen:antibody) molar 
ratio. The mixtures were incubated on a rocking plate overnight at $4{ }^{\circ} \mathrm{C}$. These mixtures were then used for immunohistochemical staining of rat and human brain tissue.

\subsubsection{Immunocytochemistry}

Mice $(N=3$, age $=3.5 \mathrm{mo}$, Balb/c strain, Jackson Labs $)$ and rats $(N=3$, age $=$ 3.5 mo, Sprague-Dawley strain, Harlan Sprague Dawley) were anesthetized with an overdose of pentobarbital and transcardially perfused with heparinized saline followed by phosphate-buffered $4 \%$ paraformaldehyde. Brains were post-fixed with phosphatebuffered $4 \%$ paraformaldehyde for $2 \mathrm{hrs}$ and then transferred to a phosphate-buffered $30 \%$ sucrose solution.

For immunocytochemistry, post-mortem brain tissue from patients with a clinical diagnosis of PD and normal human controls was obtained from the National Neurological Research Specimen Bank (Veterans Administration Medical Center [VAMC], Los Angeles, CA 90073), which is sponsored by the National Institute of Neurological Disorders and Stroke/National Institute of Mental Health (NINDS/NIMH), National Multiple Sclerosis Society, Veterans Administration Greater Los Angeles Healthcare System (11301 Wilshire Blvd., Los Angeles, CA 90073), and the Veterans Health Services and Research Administration, Department of Veterans Affairs. We analyzed two PD and control tissue blocks from three regions (midbrain, striatum, and locus ceruleus).

Tissues were sectioned on a cryostat at $20 \mu \mathrm{m}$ and collected in multiple series onto glass slides (Fisher Scientific) and circled with a PAP pen (Ted Pella, Redding, CA, USA). Human tissue sections were fixed with phosphate-buffered $4 \%$ paraformaldehyde for 30 min. Mouse, rat, normal human control, and PD tissue were subsequently processed in identical fashion for detection of $\mathrm{N}$-methyl-norsalsolinol or $\mathrm{TH}$ in individual sections and co-localization of $\mathrm{N}$-methyl-norsalsolinol with $\mathrm{TH}$, neuron-specific enolase (NSE), and glial fibrillary acidic protein (GFAP). For each block of tissue, one series of sections was stained with cresyl violet or hematoxylin and eosin for anatomical localization.

The steps for detection of $N$-methyl-norsalsolinol were as follows: (1) rinsed slides in $0.02 \mathrm{M}$ phosphate buffered saline (PBS) with $0.1 \%$ sodium azide $\left(\mathrm{NaN}_{3}\right.$; SigmaAldrich) $x 2$ over 30 min on a rotary shaker; (2) quenched endogenous peroxidases with $10 \%$ methanol and 3\% hydrogen peroxide in PBS for 5 min on a rotary shaker; (3) rinsed in PBS x 3 over 30 min on a rotary shaker; (4) blocked with $2 \%$ nonfat dry milk and $0.3 \%$ Triton X-100 (Sigma-Aldrich) for $1 \mathrm{hr}$; (5) incubated in primary antibody [mouse anti- $N$ methyl-norsalsolinol, 1:500], 3\% horse serum, and $0.1 \%$ Triton in $\mathrm{PBS} / \mathrm{NaN}_{3}$ overnight; (6) rinsed in PBS x 3 over 30 min on a rotary shaker; (7) incubated in secondary antibody [biotinylated horse anti-mouse; Vector Laboratories (Burlingame, CA, USA); $1: 500$ ], $3 \%$ horse serum, and $0.1 \%$ Triton in $\mathrm{PBS} / \mathrm{NaN}_{3}$ for $4 \mathrm{hrs}$; (8) rinsed in PBS $\times 3$ over 30 min on a rotary shaker; (9) incubated in Elite ABC reagent [Vector Laboratories] and $0.1 \%$ Triton in PBS for 75 min at room temperature; (10) rinsed in PBS $\times 3$ over 30 min on a rotary shaker; (11) subjected to a nickel-intensified diaminobenzidine [black] reaction; (12) rinsed, air-dried, dehydrated, cleared, and coverslipped. Staining for TH using monoclonal antibody 318 from Chemicon (Temecula, CA, USA) at a 1:1,000 
dilution was performed in similar fashion to help confirm localization in the substantia nigra pars compacta.

For double-label immunocytochemistry, the monoclonal antibody to $\mathrm{N}$-methylnorsalsolinol was used at a 1:250 dilution and detected with either an indocarbocyaninetagged donkey anti-mouse secondary antibody (Cy5, 1:250; Jackson ImmunoResearch Laboratories, West Grove, PA, USA) or a cyanine-tagged donkey anti-goat antibody (Cy2, 1:250; Jackson ImmunoResearch Laboratories). Primary antibodies to TH (1:250; Protos Biotech Co., New York, NY, USA), NSE (1:1500; Accurate Chemical \& Scientific Co., Westbury, NY, USA), and GFAP (1:1000; DiaSorin, Inc., Stillwater, MN, USA) were detected with either Cy2- or Cy5-tagged donkey anti-mouse antibodies.

Tissue was incubated with secondary antibodies for $3 \mathrm{hrs}$, rinsed, air-dried, dehydrated, cleared and coverslipped with 1,3 diethyl-8-phenylxanthine mounting compound (DPX; Sigma-Aldrich, St. Louis, MO, USA). Fluorescence was visualized with a confocal laser-scanning microscope (Bio-Rad, Hercules, CA, USA).

\subsection{RESULTS}

\subsubsection{Quantitative Analysis of $\mathbf{N}$-Methyl-Norsalsolinol in Brain}

With HPLC-EC, $\mathrm{N}$-methyl-norsalsolinol was detected in all rodent and human brain regions subjected to analysis. In rat and mouse, $\mathrm{N}$-methyl-norsalsolinol levels were very similar across brain regions with no apparent concentration of $N$-methylnorsalsolinol within the nigrostriatal pathways of the ventral midbrain and striatum (Table 2-1). However, in human brain, the highest concentrations of $N$-methyl-norsalsolinol were found in structures with dopamine neurons (SN) and dense dopaminergic innervation (caudate and putamen) (Table 2-2). Importantly, in comparison to normal human controls, $N$-methyl-norsalsolinol levels were significantly lower in the caudate nuclei and substantia nigra from PD patients $(P<0.05$ for both). In contrast, the difference in $\mathrm{N}$-methyl-norsalsolinol concentrations within the putamen of normal human and PD brain failed to reach statistical significance.

\subsubsection{Monoclonal Antibody to $\mathbf{N}$-Methyl-Norsalsolinol}

The competitive inhibition assay revealed that the monoclonal antibody to $\mathrm{N}$ methyl-norsalsolinol showed some cross-reactivity with norsalsolinol and slight crossreactivity with $\mathrm{N}$-methyl-salsolinol and salsolinol (Table 2-3, Figure 2-2). In contrast, cross-reactivity with other tested compounds was weak. The adsorption control experiment was consistent with a high degree of antigen-antibody specificity. $N$ methyl-norsalsolinol staining was completely eliminated whereas $\mathrm{TH}$ staining was unaffected by preincubation with $N$-methyl-norsalsolinol. 
Table 2-1. Concentration of $\mathbf{N}$-methyl-norsalsolinol within regions of rodent $\mathrm{CNS}$.

\begin{tabular}{lcc}
\hline Region & $\begin{array}{c}\text { Sprague-Dawley Rat } \\
\boldsymbol{N}=\mathbf{5}\end{array}$ & $\begin{array}{c}\text { Balb/c Mouse } \\
\boldsymbol{N}=\mathbf{5}\end{array}$ \\
\hline Frontal Cortex & $9.84+/-3.16$ & $12.88+/-1.51$ \\
Ventral Midbrain & $12.36+/-2.43$ & $10.42+/-3.34$ \\
Striatum & $10.523+/-1.95$ & $14.53+/-4.54$ \\
Hippocampus & $14.23+/-2.35$ & $18.08+/-7.87$ \\
Cerebellum & $9.54+/-2.82$ & $12.75+/-3.16$ \\
\hline
\end{tabular}

* Values are expressed as ng/g wet tissue weight +/- standard error.

Table 2-2. Concentration of $\mathbf{N}$-methyl-norsalsolinol within regions of human $\mathrm{CNS}$.

\begin{tabular}{lcc}
\hline Region & $\begin{array}{c}\text { Normal Human } \\
\boldsymbol{N}=\mathbf{5}\end{array}$ & $\begin{array}{c}\text { PD Human } \\
\boldsymbol{N}=\mathbf{5}\end{array}$ \\
\hline Frontal Cortex & $62.32+/-14.76$ & $35.77+/-16.28$ \\
Caudate Nucleus & $570.03+/-43.44$ & $105.63+/-23.55$ \\
Putamen & $314.87+/-28.73$ & $265.47+/-39.03$ \\
Substantia Nigra** & $191.17+/-12.91$ & $65.75+/-6.27$ \\
Inferior Olive & $31.54+/-9.04$ & $29.77+/-7.75$ \\
Locus Ceruleus & $18.44+/-3.66$ & $21.53+/-5.67$ \\
Hippocampus & $39.02+/-7.82$ & $46.78+/-5.95$ \\
Cerebellar Cortex & $28.15+/-7.35$ & $23.76+/-4.31$ \\
\hline
\end{tabular}

* Values are expressed as ng/g wet tissue weight +/- standard error.

** Indicates a significant difference between normal and PD human. 
Table 2-3. Percentage (\%) cross-reactivities of the monoclonal antibody with compounds structurally related to $\mathrm{N}$-methyl-norsalsolinol.

\begin{tabular}{|c|c|}
\hline Compound & $\%$ Cross-Reactivity* \\
\hline N-methyl-6,7-dihydroxytetrahydroisoquinoline & 100 \\
\hline 6,7-dihydroxytetrahydroisoquinoline & 60 \\
\hline 1,N-dimethyl-6,7-dihydroxytetrahydroisoquinoline & 15 \\
\hline 1-methyl-6,7-dihydroxytetrahydroisoquinoline & 6 \\
\hline Tetrahydroisoquinoline & $<1$ \\
\hline 5-hydroxyisoquinoline & $<1$ \\
\hline$N$-methyl-5-hydroxyisoquinolinium ion & $<1$ \\
\hline Serotonin & $<1$ \\
\hline Tetrahydropapaveroline & $<1$ \\
\hline 9H-pyrido[3,4-b]indole & $<1$ \\
\hline 1,2,3,4-tetrahydro-9H-pyrido[3,4-b]indole & $<1$ \\
\hline Dopamine & 1 \\
\hline
\end{tabular}

* The cross-reactivities were determined using the equation: \% cross-reactivity of compound $\mathrm{A}=(\mathrm{IC50}$ of $\mathrm{N}$-methyl-norsalsolinol/IC50 of compound A) $\times 100$. 


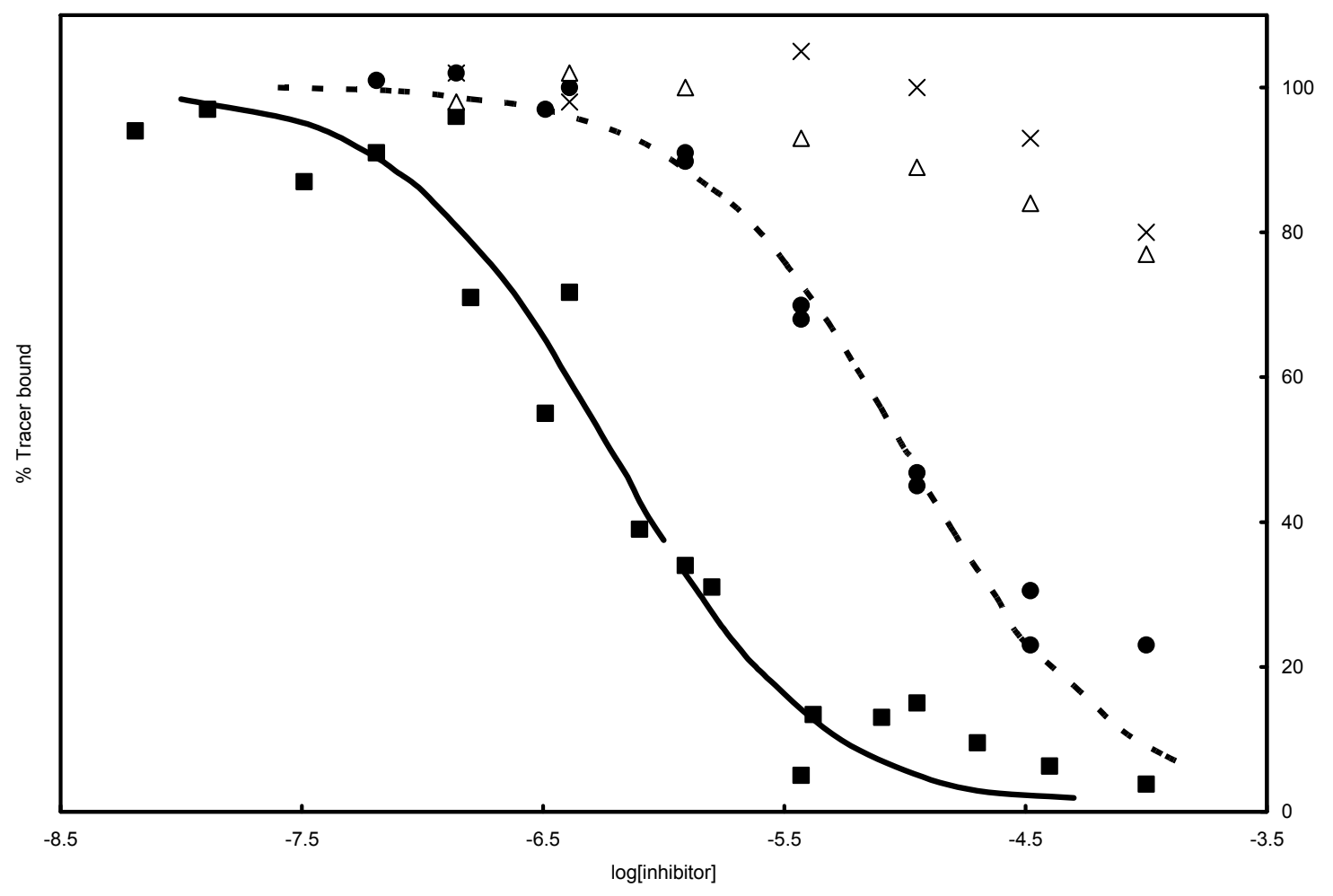

Figure 2-2. Cross-reactivities of the monoclonal antibody with compounds structurally related to $\mathbf{N}$-methyl-norsalsolinol.

Binding of the tracer (biotinylated $N$-methyl-norsalsolinol) was inhibited by $N$-methylnorsalsolinol ( $\mathbf{\square})$. The closely related compound $(R / S) 1, N$-dimethyl-norsalsolinol $(\bullet)$ produced less inhibition of tracer binding. Tetrahydroisoquinoline $(\Delta)$ and serotonin $(\mathbf{X})$ minimally inhibited tracer binding. Data points obtained with $N$-methyl-norsalsolinol and $(R / S) 1, N$-dimethyl-norsalsolinol were fitted to a simple binding isotherm. 


\subsubsection{Localization and Distribution of $\mathbf{N}$-Methyl-Norsalsolinol Immunoreactivity}

As shown in Figures 2-3 and 2-4, $\mathrm{N}$-methyl-norsalsolinol-immunoreactivity (IR) was widely distributed in rat, mouse and human brain. In rodents, the vast majority of neurons demonstrated clearly detectable $N$-methyl-norsalsolinol-IR. Within each brain area studied (striatum, midbrain, rostral medulla), neuron-to-neuron variability in $N$ methyl-norsalsolinol-IR was more notable in human than in rodent tissues.

Double-labeling studies with antibodies to GFAP and NSE demonstrated that $\mathrm{N}$ methyl-norsalsolinol-IR was limited to neurons and not present in glia. $\mathrm{N}$-methylnorsalsolinol-IR was concentrated in the soma and proximal dendritic arbors of neurons. As seen in Figures 2-3E and 2-3F, nuclear $N$-methyl-norsalsolinol-IR was much weaker than cytoplasmic $N$-methyl-norsalsolinol-IR.

Examination of cresyl violet-stained tissue along with immunocytochemistry for TH confirmed the presence of $\mathrm{N}$-methyl-norsalsolinol-IR within the substantia nigra pars compacta in both rodent and human tissue. In addition, TH-IR co-localized with $\mathrm{N}$ methyl-norsalsolinol-IR in both rodent and human tissue. In rodents, $\mathrm{N}$-methylnorsalsolinol-IR was only minimally more intense in the substantia nigra pars compacta and other dopaminergic cell groups than elsewhere in the brain. However, in normal human tissue, $\mathrm{N}$-methyl-norsalsolinol-IR was significantly more intense in the SNpc than in other cell groups.

\subsubsection{Effect of Parkinson Disease on $\mathbf{N}$-Methyl-Norsalsolinol Immunoreactivity}

As shown in Figure 2-4F-J, relative to normal control brains, $\mathrm{N}$-methylnorsalsolinol-IR was reduced in PD substantia nigra. TH-IR was markedly reduced in PD substantia nigra and localized to dystrophic neurons and dendritic processes. Lewy bodies were seen in $\mathrm{H}$ \& $\mathrm{E}$ sections from both $\mathrm{PD}$ brains used in the immunocytochemical study.

\subsection{DISCUSSION}

Several studies have suggested that isoquinolines such as the tetrahydroisoquinolines and dihydroisoquinolines may contribute to dopaminergic cell loss in PD (Kajita et al 1994; Nagatsu 1997; Ohta et al 1987; Yoshida et al 1993), data detailing the anatomical distribution of these potential neurotoxins in mammalian tissues is inconsistent and fragmentary. Using HPLC-EC and immunocytochemistry, our work describes the regional and cellular distributions of the clinically-relevant and potentially neurotoxic tetrahydroisoquinoline derivative, $N$-methyl-norsalsolinol. Our results indicate that $N$-methyl-norsalsolinol is found in neurons throughout the brain. Of utmost importance, (1) $\mathrm{N}$-methyl-norsalsolinol was concentrated in dopaminergic cell groups in human brain and (2) $N$-methyl-norsalsolinol levels were significantly decreased in dopaminergic regions of PD brain as compared to controls.

The results of this study raise several issues regarding the origin and potential neurotoxicity of isoquinolines, in general, and $\mathrm{N}$-methyl-norsalsolinol, more specifically, in the pathobiology of PD. Not limited to the dopamine-rich ventral midbrain and 

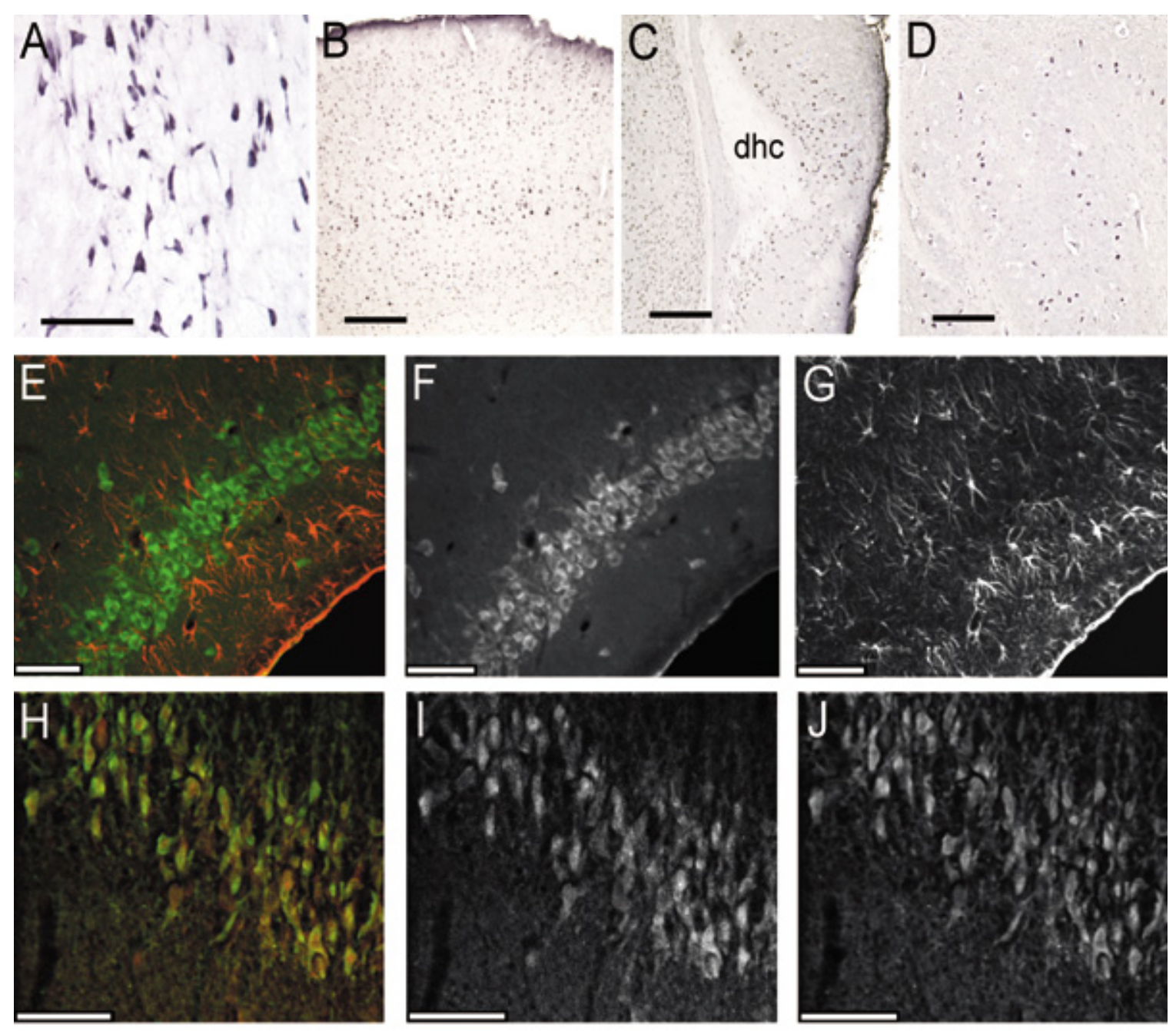

Figure 2-3. $\mathbf{N}$-methyl-norsalsolinol-IR in rodents and humans.

Examples of $\mathrm{N}$-methyl-norsalsolinol-IR are shown from rat substantia nigra pars compacta (A), rat cerebral cortex (B), mouse presubiculum (C), and human inferior olive (D). N-methyl-norsalsolinol-IR (green) did not co-localize with GFAP (red) in the CA3 region of rat hippocampus (E). Gray scale images of $N$-methyl-norsalsolinol-IR (F) and GFAP-IR (G) are shown for comparison with (E). N-methyl-norsalsolinol-IR (green) did co-localize (yellow) with NSE-IR (red) in the CA3 region of rat hippocampus $(\mathrm{H})$. Gray scale images of $N$-methyl-norsalsolinol-IR (I) and NSE-IR (J) are shown for comparison with $(\mathrm{H})$. Bars: $100 \mu \mathrm{m}$ for $\mathrm{A} ; 250 \mu \mathrm{m}$ for $\mathrm{B}, \mathrm{C}$, and D; and $50 \mu \mathrm{m}$ for $\mathrm{E}-\mathrm{J}$. 

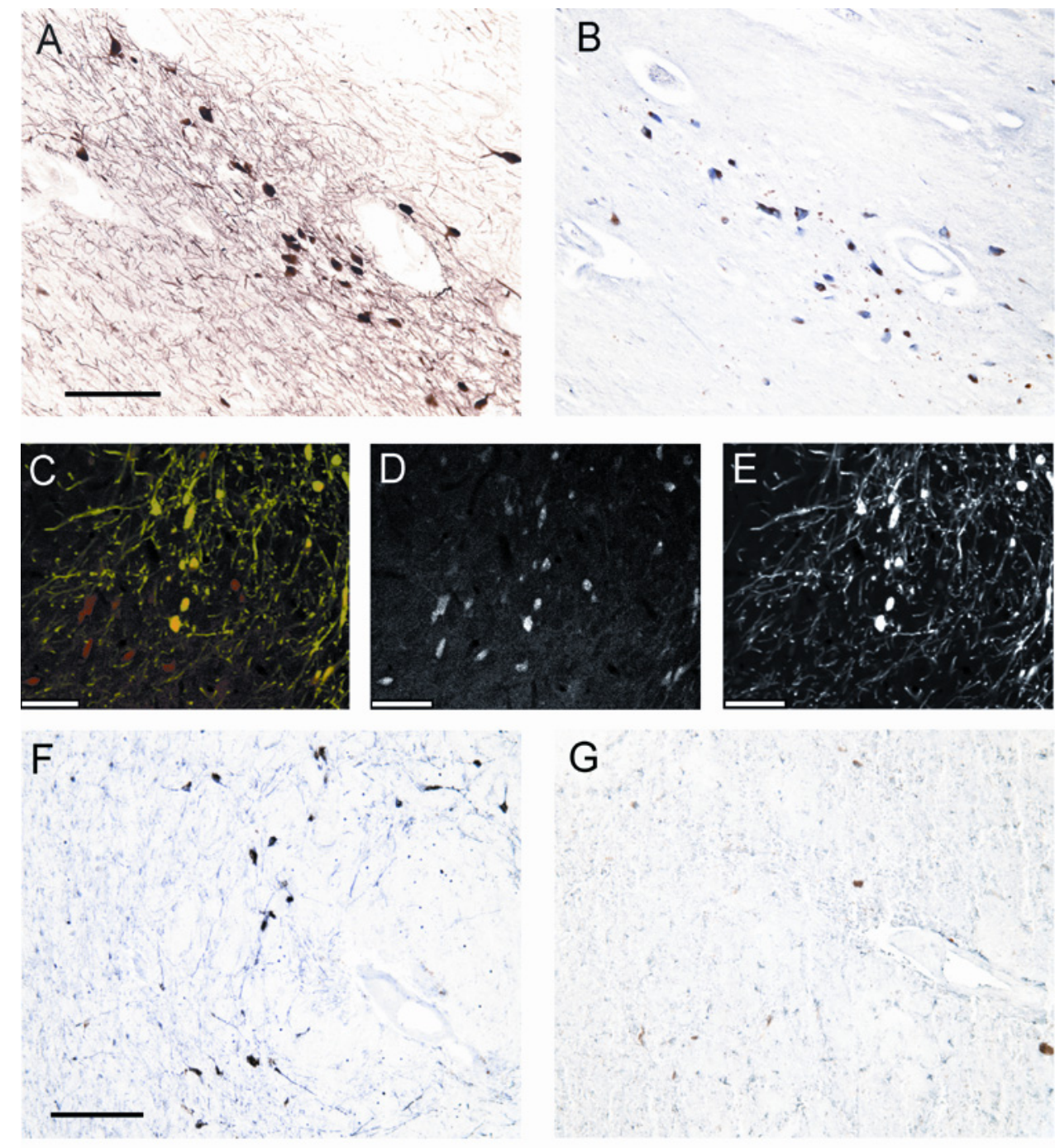

G
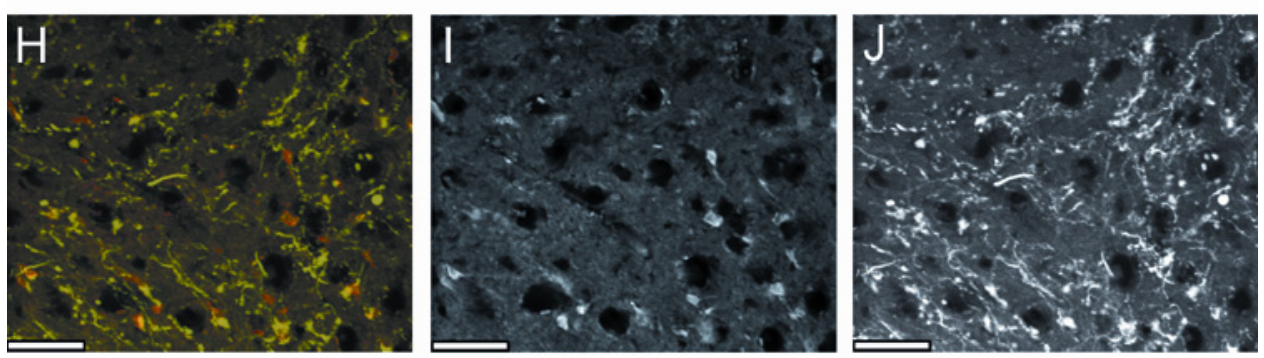

Figure 2-4. $\mathbf{N}$-methyl-norsalsolinol-IR in the Substantia Nigra.

Adjacent sections (A, B) through normal human substantia nigra showing $\mathrm{TH}-\mathrm{IR}(\mathrm{A})$ and $\mathrm{N}$-methyl-norsalsolinol-IR (B). All TH-IR (green) neurons in rat midbrain were also $\mathrm{N}$ methyl-norsalsolinol-IR (red) (C). Gray scale images of $N$-methyl-norsalsolinol-IR (D) and TH-IR (E) are shown for comparison with $C$. Adjacent sections through PD substantia nigra $(F, G)$ show that TH-IR $(F)$ was weak while $N$-methyl-norsalsolinol-IR $(\mathrm{G})$ was virtually non-existent in some regions. Confocal images $(\mathrm{H}-\mathrm{J})$ from PD substantia nigra show co-localization of $N$-methyl-norsalsolinol-IR (red) and TH-IR (green). Gray scale images of $\mathrm{N}$-methyl-norsalsolinol-IR (I) and TH-IR (J) are shown for comparison with fusion image $(H)$. 
striatum, $\mathrm{N}$-methyl-norsalsolinol was also found and quantified in cerebellum, hippocampus, and frontal cortex. Consistent with our findings, Niwa and co-workers were able to detect $\mathrm{N}$-methyl-norsalsolinol in frontal cortex using gas chromatographymass spectroscopy (Niwa et al 1991). Plausible explanations for the broad distribution of $N$-methyl-norsalsolinol include the following: $N$-methyl-norsalsolinol can be formed in cells with very low levels of dopamine and subsequently accumulate in those cells; $N$ methyl-norsalsolinol is formed by conceivable enzymatic and/or non-enzymatic biosynthetic pathways other than the one indicated in Figure 2-1C; and $\mathrm{N}$-methylnorsalsolinol (or its parent isoquinoline derivatives) is present in foods and/or beverages and crosses the blood-brain barrier (Collins et al 1990; Duncan \& Smythe 1982; Duncan et al 1984; Riggin \& Kissinger 1976; Riggin et al 1976). Data demonstrating that $N-$ methyl-norsalsolinol is able to cross the blood-brain barrier in vivo suggests that exogenous sources may contribute to its presence in brain (Thumen et al 2002). However, a potential precursor of $N$-methyl-norsalsolinol, $(R / S)$ salsolinol, does not cross the intact blood-brain barrier of young rats (Origitano et al 1981; Song et al 2006).

Unlike the situation with MPTP, our immunocytochemical data suggests that glial cells may not be critical to the potential neurotoxicity of $N$-methyl-norsalsolinol and other $\mathrm{N}$-methylated isoquinolines. Instead, $\mathrm{N}$-methylated isoquinolines like $\mathrm{N}$-methylnorsalsolinol and other closely-related tetrahydroisoquinolines may accumulate in neurons over time and subsequently lead to cell damage/death via aggregate inhibition of complex I of the mitochondrial electron transport chain. The relatively high concentrations of $\mathrm{N}$-methyl-norsalsolinol in midbrain and striatum from older humans (age $=70 \mathrm{yrs}$ ) compared to the lower concentrations in young rodents (age $=3.5 \mathrm{mo}$ ) supports the hypothesis of slow, time-dependent neuronal accumulation. Furthermore, dopaminergic regions of PD brain were found to contain lower levels of $N$-methylnorsalsolinol relative to similar regions in control human brain. This result reached statistical significance in the caudate nucleus and substantia nigra. Taken together, these findings suggest that $\mathrm{N}$-methyl-norsalsolinol is concentrated and/or synthesized from dopamine within dopaminergic neurons and subsequently released upon apoptotic lysis from the cell body (within the substantia nigra) and/or from axon terminals (within the caudate nucleus and/or putamen). In agreement with this concept, Scholz and colleagues demonstrated increased systemic $N$-methyl-norsalsolinol concentrations in patients on high-dose levodopa therapy (Scholz et al 2004). If $N$-methyl-norsalsolinol and other $\mathrm{N}$-methylated isoquinolines do, in fact, play a role in PD, then dopaminergic neurons may have relatively selective vulnerability to their toxic effects. On the other hand, cell death in PD and other neurodegenerative disorders such as Alzheimer disease is not limited to the SNpc and the widespread distribution of $N$-methylnorsalsolinol may be compatible with a global neurodegenerative process. Alternatively, $\mathrm{N}$-methyl-norsalsolinol may simply be a dopamine metabolite formed in relatively small amounts, not sufficiently toxic to be significant to the pathogenesis of PD, and released by dying dopaminergic neurons along with dopamine and its more abundant metabolites, HVA and DOPAC.

Although supplementary to the main results derived from HPLC-EC, our immunocytochemical experiments must be cautiously interpreted in the context of our competitive inhibition assay since norsalsolinol, $N$-methyl-salsolinol, and salsolinol have been detected in human brain (Musshoff et al 2000; Niwa et al 1991). These three compounds showed some evidence of cross-reactivity with the antibody generated against $\mathrm{N}$-methyl-norsalsolinol. However, the absorption control experiment indicates that there were no antigens in brain tissue with higher affinity for the antibody than $\mathrm{N}$ - 
methyl-norsalsolinol. Thus, in the broadest sense, our antibody can be viewed as a tool for localizing $N$-methyl-norsalsolinol and, to a lesser degree, closely related tetrahydroisoquinolines.

An $N$-methylated isoquinoline closely related to $N$-methyl-norsalsolinol, $N$ methyl- $(R)$ salsolinol, has also received attention as another possible endogenous dopaminergic neurotoxin. $N$-methyl- $(R)$ salsolinol is toxic to both cultured human dopaminergic cells and rat nigral dopaminergic neurons (Maruyama et al 1997a; Naoi et al 1996a). Additionally, cell death caused by $N$-methyl-(R)salsolinol in dopaminergic human neuroblastoma SH-SY5Y cells is apoptotic (Naoi et al 2000). N-methyl$(R)$ salsolinol may be synthesized in brain in a two-step pathway: (1) synthesis of $(R)$ salsolinol from dopamine and acetaldehyde mediated by salsolinol synthase, and (2) transfer of a methyl group to $(R)$ salsolinol by $\mathrm{N}$-methyl-transferase to form $\mathrm{N}$ methyl- $(R)$ salsolinol. $N$-methyl- $(R)$ salsolinol has been detected in both normal and PD CSF (Maruyama et al 1999). CSF levels of $N$-methyl-( $R)$ salsolinol were higher in newly diagnosed, untreated PD patients than in normal controls but decreased with progression of PD as has been described for $\mathrm{N}$-methyl-norsalsolinol (Maruyama et al 1999; Moser \& Kompf 1992). These results suggest that: (1) dying dopaminergic neurons release both $N$-methyl- $(R)$ salsolinol and $N$-methyl-norsalsolinol into the CSF, (2) healthy neurons release little $N$-methyl- $(R)$ salsolinol and $N$-methyl-norsalsolinol into the CSF, and (3) at later stages of PD there are too few dying cells to release significant amounts of $\mathrm{N}$-methyl-(R)salsolinol and $\mathrm{N}$-methyl-norsalsolinol into the CSF.

Limited evidence to date suggests that $N$-methyl- $(R)$ salsolinol may be a more potent neurotoxin than $N$-methyl-norsalsolinol (Naoi et al 1996a). N-methyl$(R)$ salsolinol induced Parkinsonism in rats after injection into the striatum whereas $\mathrm{N}$ methyl-norsalsolinol failed to produce an obvious behavioral effect (Naoi et al 1996a). Unfortunately, the histological effects of $N$-methyl-norsalsolinol were not compared to those of $N$-methyl-(R)salsolinol. A single injection $N$-methyl-norsalsolinol $(8 \mu \mathrm{g})$ also failed to produce overt histological damage of dopaminergic neurons after injection into the medial forebrain bundle in rats (Moser et al 1996b). In a single monkey, intraventricular injection of $\mathrm{N}$-methyl-norsalsolinol (30 mg over 4 wks) produced neither behavioral changes nor gross morphological abnormalities in the substantia nigra (Yoshida et al 1993). In contrast to the in vivo studies, $N$-methyl-norsalsolinol is nearly as toxic to cultured PC12 cells as $N$-methyl-(R)salsolinol (Maruyama et al 1993; Naoi et al 1995). Clearly, the sensitivity of in vivo neurotoxicity studies of $\mathrm{N}$-methylnorsalsolinol has been limited by sample size, dosing, injection routes and weak histological analyses. Alternatively, it is possible that $N$-methyl-norsalsolinol is not taken up by the synapses of dopaminergic neurons after intraparenchymal or intraventricular injection or that the neurotoxicity of $N$-methyl-norsalsolinol is mediated by conversion to its oxidation product, $N$-methyl-6,7-dihydroxy-isoquinolinium ion.

A substantial body of evidence suggests that the development of idiopathic PD is due to exogenous and/or endogenous toxins interacting with a genetically and/or environmentally vulnerable host. It is tempting to hypothesize that several isoquinoline toxins such as $N$-methyl-TIQ, $N$-methyl- $(R)$ salsolinol and $N$-methyl-norsalsolinol, all of which inhibit complex I of the respiratory chain, accumulate in neurons with aging and eventually lead to cell death and contribute to neurodegeneration via apoptosis. 


\subsection{CONCLUSIONS}

The isoquinoline derivative $N$-methyl-6,7-dihydroxytetrahydroisoquinoline ( $N$ methyl-norsalsolinol) is present in normal human brain and has been identified in the CSF of patients with PD. Endogenously, $N$-methyl-norsalsolinol may be derived from dopamine by condensation with aldehydes or a-ketoacids. In vitro experiments suggest that $N$-methyl-norsalsolinol is neurotoxic. In this study, HPLC-EC was used to determine $\mathrm{N}$-methyl-norsalsolinol concentrations in mouse, rat, normal human and PD brain. In addition, a monoclonal antibody was generated against $\mathrm{N}$-methyl-norsalsolinol and used to determine the cellular localization of $N$-methyl-norsalsolinol in brain. With HPLC-EC, $\mathrm{N}$-methyl-norsalsolinol was detected in all regions of rodent and human brain subjected to analysis. In rodent brains, $N$-methyl-norsalsolinol tissue concentrations were similar among frontal cortex, ventral midbrain, striatum, hippocampus and cerebellum. Conversely, in normal human control brains, $N$-methyl-norsalsolinol was concentrated in the $\mathrm{SN}$ and striatum. In comparison to normal human controls, $\mathrm{N}$-methyl-norsalsolinol levels were significantly lower in the SN and caudate nuclei from PD patients, a finding possibly related to the death of nigrostriatal dopaminergic neurons. $\mathrm{N}$-methylnorsalsolinol-immunoreactivity co-localized with a general neuronal marker (NSE) and a monoaminergic marker (TH), but not with a glial marker (GFAP). The widespread neuronal localization of $N$-methyl-norsalsolinol in several mammalian species suggests that, in isolation, this compound is a "weak" neurotoxin. However, endogeneouslyderived $\mathrm{N}$-methyl-norsalsolinol could contribute to the pathobiology of PD in genetically predisposed individuals after years of accumulation in dopaminergic neurons. 


\section{CHAPTER 3. REGIONAL DISTRIBUTION OF TETRAHYDROISOQUINOLINE DERIVATIVES IN RODENT, HUMAN, AND PARKINSON DISEASE BRAIN*}

\subsection{INTRODUCTION}

The pathological hallmark of PD is progressive degeneration of dopaminergic neurons in the SNpc. While the precise molecular mechanisms underlying the etiology of cell death in the SNpc remain unclear, oxidative stress, mitochondrial dysfunction, and environmental risk factors including exposure to neurotoxins are well documented contributors to the pathobiology of PD (Fratiglioni \& Wang 2000; Naoi et al 2002; Schapira 2001; Shavali \& Ebadi 2003). The landmark finding that an exogenous mitochondrial neurotoxin, MPTP, can cause Parkinsonism provided strong support for a neurotoxicological component to disease pathogenesis (Ballard et al 1985; Langston et al 1983). Dopaminergic cell death in PD has also been linked to excessive oxidative stress via auto- and enzymatic oxidation of dopamine (Figure 3-1) and reduced activity of complex I of mitochondrial electron transport (Hirsch \& Faucheux 1998). TIQ and its derivatives (Figures 3-1 and 3-2) comprise a family of compounds that can be derived from catecholamines, are structurally similar to MPTP, are present both endogenously in brain and exogenously in food and beverages, and have been shown to inhibit mitochondrial electron transport leading to oxidative cell injury and consequent death via apoptotic mechanisms (Maruyama et al 1997a; McNaught et al 1995b; Naoi et al 2000; Naoi et al 2004; Ogawa et al 1989; Shavali \& Ebadi 2003).

Several "endogenous" TIQ derivatives can be synthesized from dopamine or its metabolites. The catechol TIQ, 1-methyl-6,7-dihydroxy-1,2,3,4-tetrahydroisoquinoline (salsolinol; [SAL]), can be synthesized endogenously from dopamine and acetaldehyde via two distinct mechanisms (Figure 3-1). Stereoselective synthesis of the (R)enantiomer occurs via a putative salsolinol synthase (Naoi et al 1996b). SAL can also be non-enzymatically synthesized from dopamine via the Pictet-Spengler condensation reaction with acetaldehyde to yield a racemic mixture of both $(R)$ - and $(S)$-enantiomers (Cohen \& Collins 1970; Schöpf \& Bayerle 1934). Other derivatives can then be generated from SAL via the addition and/or subtraction of methyl groups via transferases (Naoi et al 1997). As a result, SAL and a number of its derivatives have been detected in brain tissue (Musshoff et al 2003; Musshoff et al 2005; Musshoff et al 2000; Musshoff et al 1999; Naoi et al 2002; Naoi et al 2004; Sjoquist et al 1982a; b; Toth et al 2001).

In addition to endogenous synthesis, TIQ derivatives may also derive from exogenous sources. For example, TIQ and many of its derivatives are natural substances in plants (Rommelspacher \& Susilo 1985) and have been detected in certain foods and beverages consumed by humans (Collins et al 1990; Duncan \& Smythe 1982; Duncan et al 1984; Makino et al 1988; Niwa et al 1989; Riggin \& Kissinger 1976; Riggin et al 1976). Several TIQ derivates have also demonstrated

\footnotetext{
* Permission to reproduce from the International Society for Neurochemistry. DeCuypere M, Lu Y, Miller DD, LeDoux MS. 2008. Regional distribution of tetrahydroisoquinoline derivatives in rodent, human, and Parkinson's disease brain. J Neurochem 107:1398413.
} 


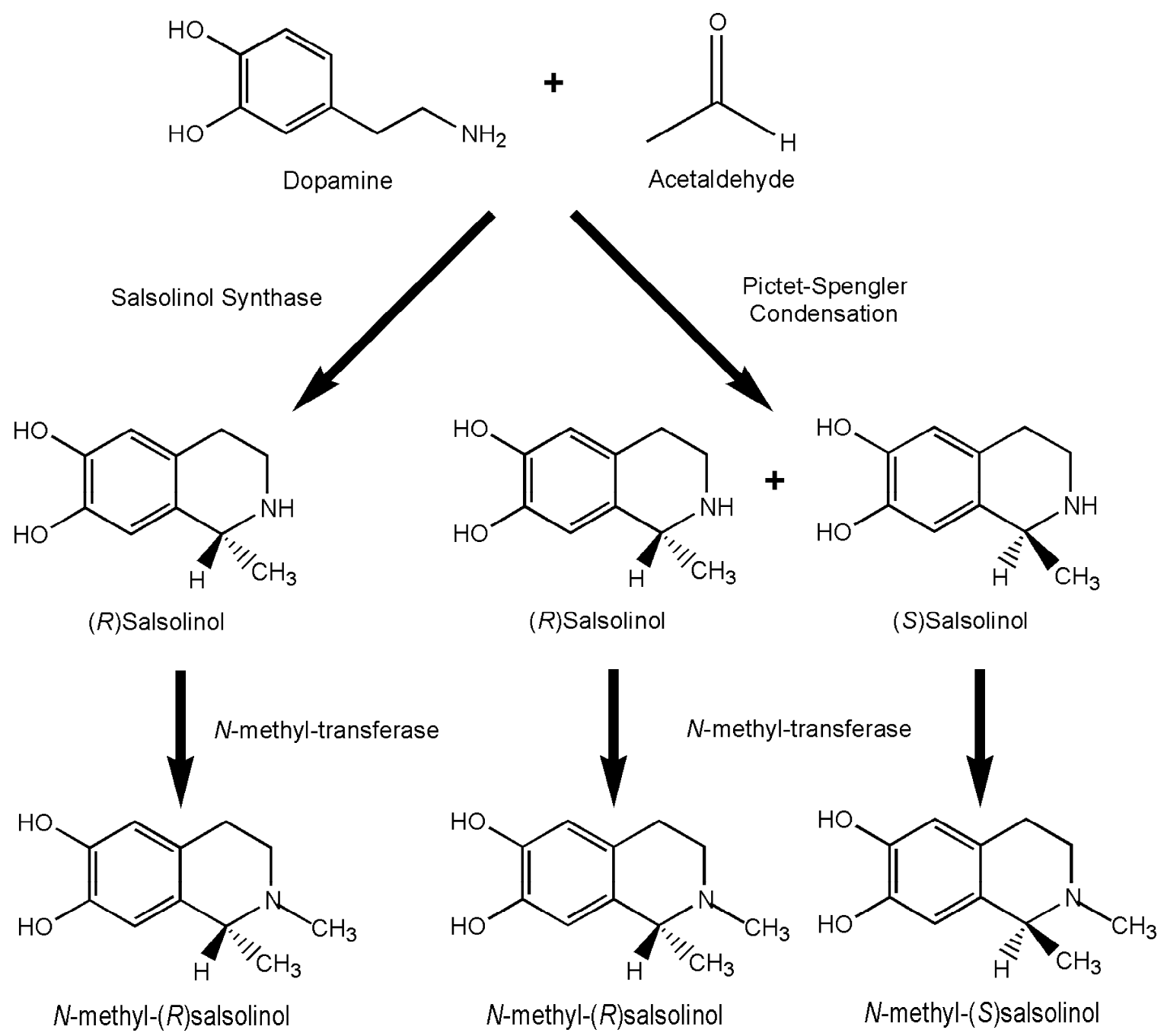

Figure 3-1. Endogenous synthesis of (R)SAL and (S)SAL.

Enzymatic formation via salsolinol synthase yields only the $(R)$-enantiomer of salsolinol, while the non-enzymatic Pictet-Spengler condensation reaction yields a racemic mixture of $(R)$ - and (S)-enantiomers. Enzymatic methylation of $(R) S A L$ and $(S) S A L$ via cellular $N$-methyl-transferases then yields the respective enantiomer of $N$-methyl-salsolinol. 
A<smiles>c1ccc2c(c1)CCNC2</smiles>

Tetrahydroisoquinoline [TIQ]<smiles>CN1CCc2ccccc2C1</smiles><smiles>CC1NCCc2ccccc21</smiles>

1-methyl-TIQ

B<smiles></smiles><smiles>Oc1cc2c(cc1O)CNCC2</smiles>

Figure 3-2. Chemical structures TIQ and its methylated (A) and benzylated (B) derivatives.

Norsalsolinol (B), a catechol TIQ derivative, is shown for structural comparison. 
blood-brain permeability (Makino et al 1988; Song et al 2006; Thumen et al 2002). These results, taken together, suggest that, in conjunction with endogenous synthesis, environmental sources may contribute to accumulation of TIQ derivatives in the nervous system.

To elucidate the potential role of TIQ neurotoxins in the pathogenesis of idiopathic PD, the tissue concentration of these compounds must be rigorously characterized. Brain distribution studies of TIQ derivatives to date have been largely incomplete, focusing on only a few regions of the brain in a single species and/or involving only a subset of TIQ derivatives, often ignoring enantiomeric considerations. Moreover, stability of TIQ derivatives has been neglected, raising concerns about the validity of previous experimental studies and questions regarding the feasibility of neuronal accumulation.

Using HPLC-EC and LC-MS/MS, the present study was undertaken to determine the regional distribution of TIQ, 1-methyl-TIQ, N-methyl-TIQ, 1-benzyl-TIQ, $(R / S) S A L, N$-methyl-(R/S)SAL, NorSAL, dopamine, DOPAC and HVA across multiple brain regions in mouse, rat, and human brain. PD brains were also analyzed to examine the relationship of TIQ neurotoxin accumulation and disease status. Finally, the stability of TIQ derivatives was investigated using thermal and freeze/thaw exposure in aqueous solution.

\subsection{MATERIALS AND METHODS}

All experiments were approved by the University of Tennessee Health Science Center Animal Care and Use Committee and performed in accordance with the National Institutes of Health Guide for the Care and Use of Laboratory Animals. Mice and rats were maintained in a temperature-controlled environment with free access to food and water.

\subsubsection{Chemicals}

Ethylenediaminetetraacetic acid (EDTA), formaldehyde, ortho-phosphoric acid, perchloric acid, sodium acetate, sodium bisulfite, sodium cyanoborohydride, triethylamine (TEA), tetramethylsilane (TMS), trifluoroacetic acid, dopamine, DOPAC, HVA, and 3,4-dihydroxybenzylamine (DHBA) were purchased from Sigma-Aldrich (St. Louis, MO, USA). Deuteriated dimethylsulfoxide (DMSO-d6) was purchased from Cambridge Isotope Labs (Woburn, MA, USA). TIQ, 1-benzyl-TIQ, (R/S)SAL, and NorSAL were purchased from Acros Organics (Geel, Belgium). 1-methyl-TIQ and Nmethyl-TIQ were purchased from AsisChem Inc. (Cambridge, MA, USA). Sodium octylsulphonate (SOS) and monobasic anhydrous sodium dihydrogen phosphate used in mobile phase preparation were purchased from Fluka Chemie (Buchs, Switzerland). HPLC grade water, acetonitrile, and $\beta$-cyclodextrin were obtained from Fisher Scientific (Hampton, NH, USA). 


\subsubsection{Synthesis of $N$-methyl-(R/S)salsolinol}

The tertiary amine $N$-methyl- $(R / S) S A L$ was prepared from the respective secondary amine $(R / S) S A L$ by reductive methylation in the presence of formaldehyde and sodium cyanoborohydride. The reaction mixture contained $0.5 \mathrm{M}$ of the secondary amine, $2.5 \mathrm{M}$ formaldehyde, $0.2 \mathrm{M}$ sodium acetate, and $1 \mathrm{M}$ sodium cyanoborohydride at $\mathrm{pH}$ 5. The reaction mixture was incubated at room temperature overnight under nitrogen in the dark. The sample was clarified by centrifugation and the clear solution was acidified with $\mathrm{HCl}$ to $\mathrm{pH} 0.1$ to decompose unreacted cyanoborohydride. The solvent was evaporated and the product was extracted from the residue with isopropyl alcohol. Evaporation of the solvent and extraction of the residue was repeated twice. The final product was stored as a solution in isopropyl alcohol at $-20^{\circ} \mathrm{C}$.

\subsubsection{Sample Preparation}

Mice $(N=5$, age $=3.5$ mo, C57BL/6 strain, Jackson Labs, Bar Harbor, Maine, USA $)$ and rats $(N=5$, age $=3.5 \mathrm{mo}$, Sprague-Dawley strain, Harlan Sprague Dawley, Inc., Indianapolis, IN, USA) were anesthetized with aerosolized isoflurane and fresh brain samples were obtained and partitioned using a dissecting microscope. Tissue samples (0.1-0.2 g) of striatum, ventral midbrain, frontal cerebral cortex, cerebellar cortex, and hippocampus were weighed and then homogenized in $100 \mu \mathrm{l}$ of ice-cold dissolution buffer ( $0.1 \mathrm{M}$ perchloric acid, $0.1 \mathrm{mM}$ sodium bisulfite, and $0.1 \mathrm{mM}$ EDTA) per $10 \mathrm{mg}$ wet weight. Cryopreserved blocks of fresh frozen post-mortem brain tissue from normal human controls $(N=5$, mean age $=70.4$ years, standard deviation $=9.4$ years $)$ and human patients with a clinical diagnosis of PD $(N=5$, mean age $=69.6$ years, standard deviation $=10.8$ years) used for HPLC-EC and LC-MS/MS analyses were obtained from the National Institute of Child Health and Human Development (NICHD) Brain and Tissue Bank for Developmental Disorders under contracts N01-HD4-3368 and N01-HD-4-3383 (Baltimore, MD, USA). Frozen blocks of caudate nucleus, putamen, substantia nigra, frontal cerebral cortex, cerebellar cortex, inferior olive, locus ceruleus and hippocampus were partitioned into 0.2-0.4 g fragments and homogenized as above. All homogenates were centrifuged at $20,000 \mathrm{~g}$ for $25 \mathrm{~min}$ at $4{ }^{\circ} \mathrm{C}$.

Supernatants were filtered through $0.22 \mu \mathrm{m}$ pore size polyvinylidene fluoride (PVDF) syringe-driven membrane filters (Millipore Corp., Bedford, MA, USA) and immediately frozen and stored at $-80^{\circ} \mathrm{C}$ until the time of analysis.

Due to the high organic content of the tissue supernatants, solid phase extraction (SPE) was performed on all samples prior to LC-MS/MS analysis. A Waters Oasis HLB extraction cartridge ( $1 \mathrm{ml} / 30 \mathrm{mg}$; Waters Corporation, Milford, MA, USA) was

conditioned with $1 \mathrm{ml}$ of methanol followed by a $1 \mathrm{ml}$ wash with water. The supernatant was loaded onto the cartridge and washed with $2 \mathrm{ml}$ deionized water and $1 \mathrm{ml} 5 \%(\mathrm{v} / \mathrm{v})$ acetonitrile with $0.1 \%(\mathrm{v} / \mathrm{v})$ trifluoroacetic acid. Analytes were eluted from the cartridge with $1 \mathrm{ml}$ of acetonitrile with $0.1 \%(\mathrm{v} / \mathrm{v})$ trifluoroacetic acid. The final solution was evaporated to dryness and the residue was reconstituted in $10 \mathrm{ml}$ of $10 \%(\mathrm{v} / \mathrm{v})$ acetonitrile. 


\subsubsection{HPLC-EC Analysis}

\subsubsection{Chromatography Standard Preparation}

Primary stock standard solutions were prepared by dissolving $10 \mathrm{mg}$ of $(R / S) S A L, N$-methyl-(R/S)SAL, NorSAL, dopamine, DOPAC and HVA in $25 \mathrm{ml}$ of dissolution buffer. These concentrates were then divided into $1 \mathrm{ml}$ aliquots, frozen, stored at $-80^{\circ} \mathrm{C}$, and thawed prior to use at $4^{\circ} \mathrm{C}$. Working standards in the $\mathrm{nM}$ range were freshly prepared prior to each assay. Standard curves employed for analyte quantification were generated with a DHBA internal standard. The relationship between concentration and relative response was linear over two orders of magnitude (sample correlation coefficient $>0.98$ ), with an overall electrochemical sensitivity of $0.001 \mu \mathrm{A}$. With HPLC-EC, the limit of quantification for all analytes was $0.7 \mathrm{ng} / \mathrm{ml}$. Instrumental limits of detection and quantification for each analyte appears in Appendix A, Table A-1.

\subsubsection{Mobile Phase Preparation}

A stock buffer solution containing $75 \mathrm{mM}$ monobasic sodium dihydrogen phosphate, $2 \mathrm{mM}$ SOS, $25 \mu \mathrm{M}$ EDTA, $20 \mathrm{mM} \beta$-cyclodextrin, and $100 \mu \mathrm{l}$ of TEA was prepared in $1800 \mathrm{ml}$ of HPLC grade water. To prepare the mobile phase, this solution was then mixed with $200 \mathrm{ml}$ of HPLC grade acetonitrile and buffered to $\mathrm{pH} 3.0$ using concentrated ortho-phosphoric acid. The mobile phase was stored at room temperature for no more than 2 days, filtered through a $0.20 \mu \mathrm{m}$ pore size white nylon filter membrane (Millipore Corp.), and degassed under vacuum for $30 \mathrm{~min}$ prior to use. The chiral mobile phase additive $\beta$-cyclodextrin is a cyclic oligosaccharide which preferentially binds to one face of an enantiomer. This binding changes the hydrogenbonding patterns of the enantiomers and allows for their separation using a standard reverse-phase column.

\subsubsection{Liquid Chromatography}

HPLC-EC analysis was performed with an ESA Model 5600A CoulArray® system (ESA Inc., Chemlsford, MA, USA), equipped with Shimadzu Model DGU-14A on-line degassing unit (Shimadzu Scientific Instruments, Columbia, MD, USA), an ESA Model 582 pump, and an ESA Model 542 refrigerated autosampler. The detection system consisted of three coulometric array modules, each containing four electrochemical detector cells. Electrode potentials were selected over the range of 0 to $+700 \mathrm{mV}$, with a $50 \mathrm{mV}$ increment against palladium electrodes. Chromatographic separation was achieved by auto-injecting $30 \mu \mathrm{l}$ sample aliquots at $5^{\circ} \mathrm{C}$ onto a MetaChem Intersil (MetaChem Technologies, Torrance, CA, USA) reversed-phase $\mathrm{C}_{18}$ column (5 $\mu \mathrm{m}$ particle size, $250 X 4.6 \mathrm{~mm}$ I.D) with an ESA Hypersil $\mathrm{C}_{18}$ pre-column (5 $\mu \mathrm{m}$ particle size, 7.5X4.6 mm I.D.) [Figure 3-3A]. A mobile phase flow rate of $1.25 \mathrm{ml} / \mathrm{min}$ and analysis time of $45 \mathrm{~min}$ were used for all experiments. System control and data acquisition/processing were performed using ESA CoulArray software (version 1.02). All samples were processed in technical triplicate with median values used for statistical analyses. 

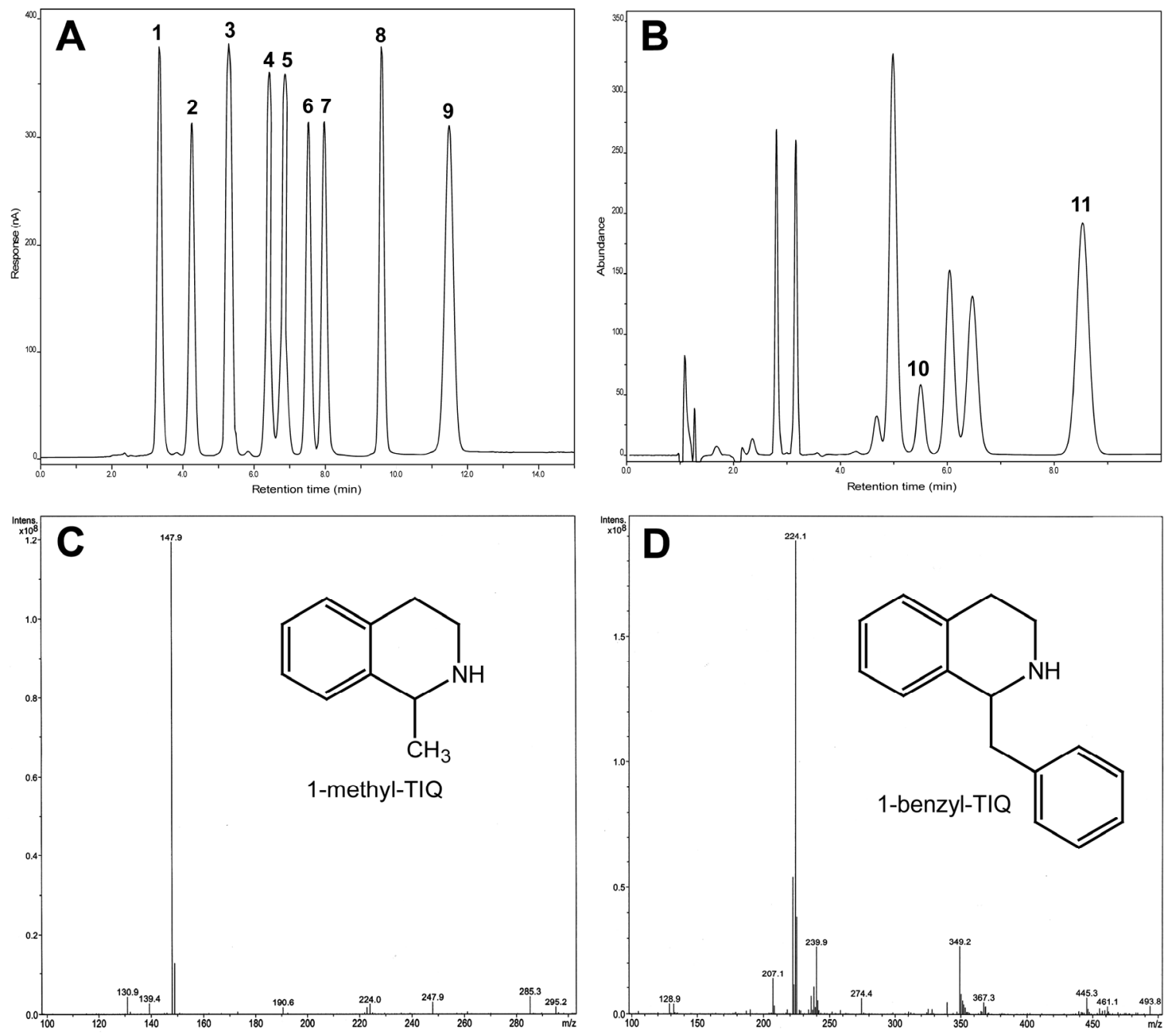

Figure 3-3. Chromatography of TIQ derivatives.

(A) HPLC-EC chromatogram of a standard solution [250 ng/ml; $150 \mathrm{mV}]$ of (1) norsalsolinol, (2) dopamine, (3) N-methyl-norsalsolinol, (4) (R)SAL, (5) (S)SAL, (6) Nmethyl-(R)SAL, (7) N-methyl-(S)SAL, (8) DOPAC, and (9) HVA used in system calibration. (B) LC-MS/MS extracted ion chromatogram [224 $\rightarrow 140 \mathrm{~m} / \mathrm{z}$ ] of C57BL/6 mouse striatum homogenate demonstrating the presence of (10) 1-methyl-TIQ and (11) 1-benzyl-TIQ, along with their respective ESI mass spectra: (C) molecular ion of 1methyl-TIQ; (D) molecular ion of 1-benzyl-TIQ. 


\subsubsection{LC-MS/MS Analysis}

\subsubsection{Chromatography Standard Preparation}

Primary stock standard solutions were prepared by dissolving $10 \mathrm{mg}$ of TIQ, 1methyl-TIQ, N-methyl-TIQ, and 1-benzyl-TIQ in $25 \mathrm{ml}$ of a mixture of $50 \%$ water and $50 \%$ acetonitrile. These concentrates were then divided into $1 \mathrm{ml}$ aliquots and stored at $-80^{\circ} \mathrm{C}$. Working standards in the $\mathrm{nM}$ range were freshly prepared prior to each assay.

\subsubsection{Liquid Chromatography}

LC-MS/MS analysis was performed using an Agilent model 1100 series HPLC system equipped with a Bruker Esquire-LC ion trap tandem mass selective detector (Bruker Daltronics Inc., Billerica, MA, USA), a Model 1327A temperature controlled autosampler, a Model 1316A temperature controlled column compartment, and a Model G1312A binary pump with Model G1322A solvent degassing module, and a Model G1314A variable wavelength UV-vis detector (Agilent Technologies, Santa Clara, CA). Ultraviolet absorbance monitoring was performed at $220 \mathrm{~nm}$. Separation was achieved by auto-injecting $10 \mu \mathrm{l} \mathrm{sample} \mathrm{aliquots} \mathrm{at} 5^{\circ} \mathrm{C}$ onto a Thermo Hypersil-Keystone reversed-phase $\mathrm{C}_{18}$ column $(5 \mu \mathrm{m} ; 50 \times 2.1 \mathrm{~mm}$ I.D.; Thermo Fisher Scientific, Waltham, MA, USA) in combination with a $\mathrm{C}_{18}$ Hypersil guard column (10 x $2.1 \mathrm{~mm}$ I.D.)

[Figure 3-3B]. A binary mobile phase flow rate of $0.7 \mathrm{ml} / \mathrm{min}$ and analysis time of $25 \mathrm{~min}$ was utilized. Mobile phase A consisted of water with $0.1 \%$ formic acid and mobile phase $B$ consisted of acetonitrile with $0.1 \%$ formic acid. The following mobile phase gradient was used: $0 \mathrm{~min} A / B=95: 5,5 \mathrm{~min} A / B=70: 30,10 \mathrm{~min} A / B=50: 50,15 \mathrm{~min} A / B=70: 30$, $20 \mathrm{~min} A / B=95: 5$. The column was given a 5 min post-run period to allow for reequilibration. System control and data acquisition/processing were performed using ChemStation software revision A.08.03 for LC-MS systems (Agilent Technologies).

\subsubsection{Ion Trap Tandem Mass Spectrometry}

Full-scan mode was used to acquire mass spectra, precursor ions, and product ions. EsquireControl software version 6.16 (Bruker Daltronics Inc.) was employed for control of all mass spectrometric conditions. The mass spectrometer was equipped with a heated capillary interface and an electrospray ionization (ESI) source, operating in positive ion mode. The ESI source and MS/MS parameters were automatically optimized. Spray needle voltage was $4.0 \mathrm{kV}$ and capillary temperature was set to $220^{\circ} \mathrm{C}$. Sheath and auxiliary nitrogen gas flow were set to 40 and 0 psi, respectively. Automatic gain control was utilized with a maximum isolation time of $300 \mathrm{~ms}$. A relative collision energy of $33 \%$ was used for all analyses. The product ions producing the highest intensity were used for quantification to increase analytical sensitivity and selectivity in LC-MS/MS mode (Figure 3-3C-D). Quantification was based on detector response, defined as the ratio of the base peak ion of interest to the base peak ion of the standard compound. The relationship between concentration and relative response was linear over two orders of magnitude (sample correlation coefficient $>0.998$ ) for the $\mathrm{MS} / \mathrm{MS}$ procedure. All samples were processed in technical triplicate with median values used for statistical analyses. With LC-MS/MS, $0.3 \mathrm{ng} / \mathrm{ml}$ was the limit of 
quantification for all analytes. Instrumental limits of detection and quantification for each analyte appears in Appendix A, Table A-1.

\subsubsection{Compound Stability Experiments}

All compounds were subjected to freeze/thaw and thermal stability testing under closed conditions. A $10 \mathrm{mg}$ sample of each compound was weighted and dissolved in

DMSO-d6. Four sets of sealed tubes were exposed to either three freeze-thaw cycles ($80{ }^{\circ} \mathrm{C} / 22^{\circ} \mathrm{C}$ ) or a maximum of 28 days of heat exposure at $22^{\circ} \mathrm{C}, 37^{\circ} \mathrm{C}$, and $60{ }^{\circ} \mathrm{C}$, respectively. To assess structural integrity after each freeze/thaw cycle and 7-day heat exposure interval, ${ }^{1} \mathrm{H}$ NMR spectra were recorded on a Bruker AX 300 spectrometer (Bruker Daltronics Inc.) at $300 \mathrm{MHz}$ using TMS as an internal standard.

\subsubsection{Statistical Analysis}

Statistical analyses were based on median values derived from technical triplicates. Enantiomeric, methylation and TIQ derivative/dopamine ratios were then determined for each individual sample. Concentrations derived from all HPLC-EC and LC-MS/MS experiments were analyzed with individual Student's $t$-tests. Student's $t$ tests were also utilized to examine TIQ derivative/dopamine ratios and the hypotheses that regional enantiomeric and methylation ratios differed from unity (1:1). An alpha $(\alpha)$ of 0.05 was chosen for statistical significance. Holm's method was used to correct for multiple comparisons (Holm 1979).

\subsection{RESULTS}

\subsubsection{Quantitative Analysis of TIQ Derivatives in Brain}

Using HPLC-EC and LC-MS/MS, TIQ derivatives were detected in all rodent and human brain regions subjected to analysis. Analysis of Tables 3-1 and 3-2 reveals several general patterns and trends in the tissue distribution of TIQ derivatives. First of all, TIQ was present at higher concentrations than its methylated and benzylated derivatives (1-methyl-TIQ, N-methyl-TIQ, and 1-benzyl-TIQ) in all brain regions. Second, TIQ and its methylated and benzylated derivatives were found at lower concentrations than $(R / S) S A L$ and its derivatives in all species. Lastly, the tissue concentrations of NorSAL tended to be intermediate between the concentrations of TIQ and $(R / S) S A L$.

In rat and mouse, the levels of many $\mathrm{TIQ}$ derivatives were very similar across brain regions with no apparent differences in concentrations (Table 3-1). For example, the regional concentrations of TIQ, 1-methyl-TIQ, N-methyl-TIQ, and 1-benzyl-TIQ exhibited minimal variation across the brain. However, in the striatum, the concentrations of both $N$-methyl- $(R) S A L$ and $N$-methyl-(S)SAL were significantly higher in mice $(P<0.0008)$. In contrast, $N$-methyl- $(S) S A L$ content of the ventral midbrain was significantly higher in the rat $(P<0.0008)$. While analysis of rat ventral midbrain revealed greater dopamine content, NorSAL concentration was decreased in this region as compared to the mouse $(P<0.0008)$. The trend of increased dopamine metabolite 
Table 3-1. Regional distribution of TIQ derivatives in rodent brain.

\begin{tabular}{|c|c|c|c|c|}
\hline \multirow{2}{*}{ Compound } & \multicolumn{2}{|c|}{ SD Rat Striatum } & \multicolumn{2}{|c|}{ C57BL6 Mouse Striatum } \\
\hline & $\mathrm{ng} / \mathrm{g}$ & $\mathrm{nM}$ & $\mathrm{ng} / \mathrm{g}$ & $\mathrm{nM}$ \\
\hline TIQ & $4.64+/-1.19$ & $0.033+/-0.009$ & $5.58+/-1.2$ & $0.042+/-0.009$ \\
\hline 1-Me-TIQ & $1.06+/-0.30$ & $0.007+/-0.002$ & $2.00+/-0.76$ & $0.014+/-0.005$ \\
\hline N-Me-TIQ & $0.88+/-0.24$ & $0.006+/-0.002$ & $1.22+/-0.27$ & $0.008+/-0.002$ \\
\hline 1-Benzyl-TIQ & $2.19+/-0.92$ & $0.010+/-0.004$ & $4.12+/-1.11$ & $0.018+/-0.005$ \\
\hline (R)SAL & $28.00+/-3.27$ & $0.16+/-0.02$ & $122.50+/-30.33$ & $0.684+/-0.17$ \\
\hline (S)SAL & $24.50+/-1.79$ & $0.14+/-0.01$ & $106.45+/-22.95$ & $0.59+/-0.13$ \\
\hline N-Me-(R)SAL & $19.98+/-2.60$ * & $0.13+/-0.01$ * & $100.40+/-8.13$ * & $0.52+/-0.04$ * \\
\hline N-Me-(S)SAL & $17.38+/-1.45$ * & $0.090+/-0.008$ * & $92.07+/-6.55^{*}$ & $0.47+/-0.03$ * \\
\hline NorSAL & $5.13+/-1.34$ & $0.031+/-0.008$ & $5.19+/-1.74$ & $0.03+/-0.01$ \\
\hline N-Me-NorSAL & $10.52+/-1.95$ & $0.06+/-0.01$ & $13.85+/-1.07$ & $0.077+/-0.006$ \\
\hline Dopamine & $2347.95+/-59.95$ & $15.33+/-0.39$ & $2114.19+/-47.24$ & $13.80+/-0.31$ \\
\hline DOPAC & $225.45+/-10.60$ & $1.34+/-0.06$ & $221.12+/-18.50$ & $1.31+/-0.11$ \\
\hline HVA & $183.06+/-8.51$ & $1.00+/-0.05$ & $177.02+/-21.56$ & $0.97+/-0.12$ \\
\hline \multirow{2}{*}{ Compound } & \multicolumn{2}{|c|}{ SD Rat Ventral Midbrain } & \multicolumn{2}{|c|}{ C57BL6 Mouse Ventral Midbrain } \\
\hline & $\mathrm{ng} / \mathrm{g}$ & $\mathrm{nM}$ & $\mathrm{ng} / \mathrm{g}$ & $\mathrm{nM}$ \\
\hline TIQ & $5.43+/-1.14$ & $0.041+/-0.009$ & $7.18+/-1.22$ & $0.054+/-0.009$ \\
\hline 1-Me-TIQ & $1.13+/-0.57$ & $0.008+/-0.004$ & $2.52+/-0.43$ & $0.017+/-0.003$ \\
\hline N-Me-TIQ & $0.82+/-0.27$ & $0.006+/-0.002$ & $1.73+/-0.21$ & $0.012+/-0.001$ \\
\hline 1-Benzyl-TIQ & $3.39+/-1.20$ & $0.015+/-0.005$ & $2.14+/-0.31$ & $0.010+/-0.001$ \\
\hline (R)SAL & $211.02+/-13.76$ & $1.18+/-0.08$ & $148.82+/-11.73$ & $0.83+/-0.06$ \\
\hline (S) SAL & $170.89+/-18.89$ & $0.95+/-0.10$ & $80.25+/-22.27$ & $0.45+/-0.12$ \\
\hline N-Me-(R)SAL & $240.90+/-15.75$ & $1.25+/-0.08$ & $215.58+/-8.55$ & $1.12+/-0.04$ \\
\hline N-Me-(S)SAL & $233.50+/-4.49$ * & $1.21+/-0.02$ * & $194.84+/-3.71$ * & $1.00+/-0.02$ * \\
\hline NorSAL & $6.40+/-1.04$ * & $0.039+/-0.006$ * & $24.04+/-2.03$ * & $0.15+/-0.01$ * \\
\hline N-Me-NorSAL & $12.36+/-2.43$ & $0.07+/-0.01$ & $43.93+/-5.99$ & $0.24+/-0.03$ \\
\hline Dopamine & $2363.49+/-28.08$ * & $15.43+/-0.18$ * & $1248.38+/-19.02$ * & $8.15+/-0.12$ * \\
\hline DOPAC & $62.76+/-3.63$ & $0.37+/-0.02$ & $61.29+/-5.22$ & $0.364+/-0.03$ \\
\hline HVA & $50.87+/-6.39$ & $0.28+/-0.03$ & $45.54+/-8.21$ & $0.25+/-0.04$ \\
\hline \multirow{2}{*}{ Compound } & \multicolumn{2}{|c|}{ SD Rat Frontal Cortex } & \multicolumn{2}{|c|}{ C57BL6 Mouse Frontal Cortex } \\
\hline & $\mathrm{ng} / \mathrm{g}$ & $\mathrm{nM}$ & $\mathrm{ng} / \mathrm{g}$ & $\mathrm{nM}$ \\
\hline$\overline{T I Q}$ & $7.32+/-1.91$ & $0.05+/-0.01$ & $10.19+/-1.57$ & $0.08+/-0.01$ \\
\hline 1-Me-TIQ & $3.20+/-0.93$ & $0.022+/-0.006$ & $1.89+/-0.33$ & $0.013+/-0.002$ \\
\hline N-Me-TIQ & $1.70+/-0.63$ & $0.012+/-0.004$ & $2.03+/-0.71$ & $0.014+/-0.005$ \\
\hline 1-Benzyl-TIQ & $0.93+/-0.36$ & $0.004+/-0.002$ & $2.52+/-0.63$ & $0.011+/-0.003$ \\
\hline (R)SAL & $15.08+/-4.55$ & $0.08+/-0.02$ & $23.48+/-1.40$ & $0.131+/-0.008$ \\
\hline (S)SAL & $4.18+/-1.29$ & $0.023+/-0.007$ & $17.58+/-6.22$ & $0.10+/-0.03$ \\
\hline $\mathrm{N}-\mathrm{Me}-(\mathrm{R}) \mathrm{SAL}$ & $45.64+/-6.08$ & $0.24+/-0.03$ & $12.66+/-6.98$ & $0.07+/-0.04$ \\
\hline $\mathrm{N}-\mathrm{Me}-(\mathrm{S}) \mathrm{SAL}$ & $25.19+/-3.34$ & $0.13+/-0.02$ & $8.32+/-1.14$ & $0.043+/-0.006$ \\
\hline NorSAL & $5.22+/-0.71$ & $0.032+/-0.004$ & $4.22+/-0.93$ & $0.026+/-0.006$ \\
\hline N-Me-NorSAL & $9.84+/-3.16$ & $0.05+/-0.02$ & $15.54+/-2.16$ & $0.09+/-0.01$ \\
\hline Dopamine & $545.22+/-42.83$ & $3.56+/-0.28$ & $728.17+/-38.34$ & $4.75+/-0.25$ \\
\hline DOPAC & $273.45+/-15.45$ & $1.63+/-0.09$ & $244.08+/-25.86$ & $1.45+/-0.15$ \\
\hline HVA & $232.96+/-24.61$ & $1.28+/-0.13$ & $167.70+/-14.03$ & $0.92+/-0.08$ \\
\hline \multirow{2}{*}{ Compound } & \multicolumn{2}{|c|}{ SD Rat Hippocampus } & \multicolumn{2}{|c|}{ C57BL6 Mouse Hippocampus } \\
\hline & $\mathrm{ng} / \mathrm{g}$ & $\mathrm{nM}$ & $\mathrm{ng} / \mathrm{g}$ & $\mathrm{nM}$ \\
\hline TIQ & $6.75+/-1.40$ & $0.05+/-0.01$ & $6.04+/-1.89$ & $0.04+/-0.01$ \\
\hline 1-Me-TIQ & $2.32+/-0.90$ & $0.016+/-0.006$ & $5.88+/-1.55$ & $0.04+/-0.01$ \\
\hline N-Me-TIQ & $3.46+/-1.04$ & $0.024+/-0.007$ & $1.31+/-0.81$ & $0.009+/-0.006$ \\
\hline 1-Benzyl-TIQ & $5.29+/-1.44$ & $0.024+/-0.006$ & $4.55+/-1.28$ & $0.020+/-0.006$ \\
\hline (R)SAL & $12.10+/-2.50$ * & $0.07+/-0.01$ * & $34.20+/-1.55$ * & $0.191+/-0.009$ * \\
\hline (S)SAL & $6.38+/-1.70$ & $0.036+/-0.009$ & $19.30+/-2.51$ & $0.11+/-0.01$ \\
\hline N-Me-(R)SAL & $7.07+/-1.10$ & $0.037+/-0.006$ & $8.75+/-1.09$ & $0.045+/-0.006$ \\
\hline N-Me-(S)SAL & $5.50+/-0.74$ & $0.028+/-0.004$ & $3.19+/-0.31$ & $0.017+/-0.002$ \\
\hline NorSAL & $8.38+/-1.52$ & $0.051+/-0.009$ & $6.57+/-2.06$ & $0.04+/-0.01$ \\
\hline N-Me-NorSAL & $14.23+/-2.35$ & $0.08+/-0.01$ & $18.51+/-5.72$ & $0.10+/-0.03$ \\
\hline Dopamine & $54.54+/-9.52$ & $0.36+/-0.06$ & $66.78+/-6.85$ & $0.44+/-0.04$ \\
\hline DOPAC & $20.42+/-2.34$ * & $0.12+/-0.01$ * & $67.55+/-3.43$ * & $0.40+/-0.02 *$ \\
\hline HVA & $26.57+/-5.78$ & $0.15+/-0.03$ & $65.00+/-8.20$ & $0.36+/-0.04$ \\
\hline
\end{tabular}


Table 3-1 (Continued).

\begin{tabular}{|c|c|c|c|c|}
\hline \multirow{2}{*}{ Compound } & \multicolumn{2}{|c|}{ SD Rat Cerebellum } & \multicolumn{2}{|c|}{ C57BL6 Mouse Cerebellum } \\
\hline & $\mathrm{ng} / \mathrm{g}$ & $\mathrm{nM}$ & $\mathrm{ng} / \mathrm{g}$ & $\mathrm{nM}$ \\
\hline TIQ & $3.03+/-0.90$ & $0.023+/-0.007$ & $4.12+/-0.32$ & $0.031+/-0.002$ \\
\hline 1-Me-TIQ & $0.79+/-0.21$ & $0.005+/-0.001$ & $1.24+/-0.21$ & $0.008+/-0.001$ \\
\hline N-Me-TIQ & $1.92+/-0.46$ & $0.013+/-0.003$ & $0.69+/-0.13$ & $0.005+/-0.001$ \\
\hline 1-Benzyl-TIQ & $0.88+/-0.16$ & $0.004+/-0.001$ & $1.24+/-0.27$ & $0.006+/-0.001$ \\
\hline (R)SAL & $12.26+/-2.53$ & $0.07+/-0.01$ & $16.84+/-1.20$ & $0.094+/-0.007$ \\
\hline (S)SAL & $7.28+/-2.07$ & $0.04+/-0.01$ & $9.65+/-1.85$ & $0.05+/-0.01$ \\
\hline N-Me-(R)SAL & $15.80+/-2.85$ & $0.08+/-0.01$ & $17.10+/-3.86$ & $0.09+/-0.02$ \\
\hline N-Me-(S)SAL & $8.93+/-2.65$ & $0.05+/-0.01$ & $3.52+/-0.43$ & $0.018+/-0.002$ \\
\hline NorSAL & $5.40+/-1.65$ & $0.03+/-0.01$ & $8.49+/-1.32$ & $0.052+/-0.008$ \\
\hline N-Me-NorSAL & $9.54+/-2.82$ & $0.05+/-0.02$ & $22.44+/-4.96$ & $0.12+/-0.03$ \\
\hline Dopamine & $43.62+/-3.46$ & $0.28+/-0.0$ & $38.81+/-4.56$ & $0.253+/-0.030$ \\
\hline DOPAC & $15.59+/-2.77$ & $0.09+/-0.02$ & $17.74+/-2.55$ & $0.11+/-0.01$ \\
\hline HVA & $22.94+/-4.56$ & $0.13+/-0.02$ & $13.12+/-2.98$ & $0.07+/-0.02$ \\
\hline
\end{tabular}

Values are expressed as $\mathrm{ng} / \mathrm{g}$ wet tissue weight + /- standard error. * Significant difference between groups. 
Table 3-2. Regional distribution of TIQ derivatives in normal human and PD brain.

\begin{tabular}{|c|c|c|c|c|}
\hline \multirow{2}{*}{ Compound } & \multicolumn{2}{|c|}{ Normal Human Caudate } & \multicolumn{2}{|c|}{ PD Human Caudate } \\
\hline & $\mathrm{ng} / \mathrm{g}$ & $\mathrm{nM}$ & $\mathrm{ng} / \mathrm{g}$ & $\mathrm{nM}$ \\
\hline TIQ & $2.84+/-0.82$ & $0.021+/-0.006$ & $3.81+/-1.10$ & $0.029+/-0.008$ \\
\hline 1-Me-TIQ & $1.33+/-0.77$ & $0.009+/-0.005$ & $2.00+/-0.21$ & $0.014+/-0.001$ \\
\hline N-Me-TIQ & $0.93+/-0.25$ & $0.006+/-0.002$ & $1.40+/-0.36$ & $0.010+/-0.002$ \\
\hline 1-Benzyl-TIQ & $1.37+/=0.41$ & $0.006+/-0.002$ & $1.79+/-0.27$ & $0.008+/-0.001$ \\
\hline (R)SAL & $393.40+/-10.95$ * & $2.19+/-0.06 *$ & $255.79+/-18.66$ * & $1.43+/-0.10 *$ \\
\hline (S)SAL & $394.38+/-15.51$ * & $2.20+/-0.09 *$ & $249.69+/-17.34$ * & $1.39+/-0.10^{*}$ \\
\hline N-Me-(R)SAL & $272.06+/-20.58$ * & $1.41+/-0.11$ * & $90.30+/-13.98$ * & $0.47+/-0.07$ * \\
\hline N-Me-(S)SAL & $281.92+/-13.91$ * & $1.46+/-0.07$ * & $83.57+/-14.06$ * & $0.43+/-0.07^{*}$ \\
\hline NorSAL & $65.97+/-5.96$ & $0.40+/-0.04$ & $50.17+/-3.01$ & $0.31+/-0.02$ \\
\hline N-Me-NorSAL & $570.03+/-43.44$ * & $3.18+/-0.24$ * & $105.63+/-23.55^{*}$ & $0.59+/-0.13^{*}$ \\
\hline Dopamine & $6944.17+/-32.81$ * & $45.33+/-0.21 *$ & $1295.59+/-80.20$ * & $8.46+/-0.52$ * \\
\hline DOPAC & $1204.95+/-85.28$ & $7.17+/-0.51$ & $786.95+/-67.55$ & $4.68+/-0.40$ \\
\hline HVA & $2179.01+/-71.75$ * & $11.96+/-0.39$ * & $1082.70+/-48.66$ * & $5.94+/-0.27$ * \\
\hline \multirow{2}{*}{ Compound } & \multicolumn{2}{|c|}{ Normal Human Putamen } & \multicolumn{2}{|c|}{ PD Human Putamen } \\
\hline & $\mathrm{ng} / \mathrm{g}$ & $\mathrm{nM}$ & $\mathrm{ng} / \mathrm{g}$ & $\mathrm{nM}$ \\
\hline TIQ & $3.49+/-0.97$ & $0.026+/-0.007$ & $4.45+/-1.31$ & $0.03+/-0.01$ \\
\hline 1-Me-TIQ & $2.12+/-0.68$ & $0.014+/-0.005$ & $1.62+/=0.81$ & $0.011+/-0.006$ \\
\hline N-Me-TIQ & $1.90+/-0.42$ & $0.013+/-0.003$ & $1.34+/=0.24$ & $0.009+/-0.002$ \\
\hline 1-Benzyl-TIQ & $1.42+/-0.36$ & $0.006+/-0.002$ & $0.73+/-0.16$ & $0.003+/-0.001$ \\
\hline (R)SAL & $593.68+/-32.38$ & $3.31+/-0.18$ & $379.44+/-23.74$ & $2.12+/-0.13$ \\
\hline (S)SAL & $591.87+/-32.04$ & $3.30+/-0.18$ & $347.65+/-36.91$ & $1.94+/-0.21$ \\
\hline N-Me-(R)SAL & $383.03+/-31.31$ & $1.98+/-0.16$ & $341.20+/-27.87$ & $1.77+/-0.14$ \\
\hline N-Me-(S)SAL & $376.81+/-17.68$ & $1.95+/-0.09$ & $358.01+/-43.85$ & $1.85+/-0.23$ \\
\hline NorSAL & $109.59+/-15.04$ & $0.67+/-0.09$ & $50.21+/-4.09$ & $0.31+/-0.02$ \\
\hline N-Me-NorSAL & $314.87+/-28.73$ & $1.76+/-0.16$ & $265.47+/-39.03$ & $1.48+/-0.22$ \\
\hline Dopamine & $4142.33+/-38.88$ * & $27.04+/-0.25$ * & $1639.81+/-30.53$ * & $10.70+/-0.20$ * \\
\hline DOPAC & $386.16+/-15.47$ * & $2.30+/-0.09 *$ & 152.17 +/- 8.08 * & $0.90+/-0.05$ * \\
\hline HVA & $1014.04+/-18.41$ * & $5.57+/-0.10 *$ & $358.13+/-9.80$ * & $1.97+/-0.05$ * \\
\hline \multirow{2}{*}{ Compound } & \multicolumn{2}{|c|}{ Normal Human Substantia Nigra } & \multicolumn{2}{|c|}{ PD Human Substantia Nigra } \\
\hline & $\mathrm{ng} / \mathrm{g}$ & $\mathrm{nM}$ & $\mathrm{ng} / \mathrm{g}$ & $\mathrm{nM}$ \\
\hline TIQ & $4.48+/-0.91$ & $0.034+/-0.007$ & $3.97+/-0.92$ & $0.030+/-0.007$ \\
\hline & /- 0.17 & 001 & $5+/-0.88$ & $0.014+/-0.006$ \\
\hline N-Me-TIQ & $2.16+/-0.30$ & $0.015+/-0.002$ & $1.83+/-0.67$ & $0.012+/-0.005$ \\
\hline 1-Benzyl-TIQ & $1.49+/-0.42$ & $0.007+/-0.002$ & $0.90+/-0.19$ & $0.004+/-0.001$ \\
\hline (R)SAL & $204.79+/-21.91$ & $1.14+/-0.12$ & $221.32+/-15.90$ & $1.23+/-0.09$ \\
\hline (S)SAL & $213.19+/-25.83$ & $1.19+/-0.14$ & $210.88+/-17.73$ & $1.18+/-0.10$ \\
\hline N-Me-(R)SAL & $174.92+/-19.42$ * & $0.90+/-0.10$ * & $61.44+/-6.26$ * & $0.32+/-0.03$ * \\
\hline N-Me-(S)SAL & $187.22+/-24.54$ & $0.97+/-0.13$ & $56.25+/-5.67$ & $0.29+/-0.03$ \\
\hline NorSAL & $62.24+/-7.55$ & $0.38+/-0.05$ & $54.60+/-9.54$ & $0.33+/-0.06$ \\
\hline N-Me-NorSAL & $191.17+/-12.91$ * & $1.07+/-0.07$ * & $29.77+/-7.75$ * & $0.17+/-0.04$ * \\
\hline Dopamine & $1432.05+/-26.56$ * & $9.35+/-0.17^{*}$ & $114.42+/-11.81$ * & $0.75+/-0.08$ * \\
\hline DOPAC & $945.17+/-36.82$ * & $5.62+/-0.22$ * & $90.12+/-21.42$ * & $0.54+/-0.13$ * \\
\hline HVA & $1276.78+/-11.79$ * & $7.01+/-0.06$ * & $84.44+/-11.31$ * & $0.46+/-0.06$ * \\
\hline \multirow{2}{*}{ Compound } & \multicolumn{2}{|c|}{ Normal Human Locus Ceruleus } & \multicolumn{2}{|c|}{ PD Human Locus Ceruleus } \\
\hline & $\mathrm{ng} / \mathrm{g}$ & $\mathrm{nM}$ & $\mathrm{ng} / \mathrm{g}$ & $\mathrm{nM}$ \\
\hline$\overline{T I Q}$ & $5.61+/-1.81$ & $0.04+/-0.01$ & $4.92+/-1.05$ & $0.037+/-0.008$ \\
\hline 1-Me-TIQ & $2.03+/-0.25$ & $0.014+/-0.002$ & $1.86+/-0.61$ & $0.013+/-0.004$ \\
\hline N-Me-TIQ & $1.85+/-0.40$ & $0.013+/-0.003$ & $1.74+/-0.55$ & $0.012+/-0.004$ \\
\hline 1-Benzyl-TIQ & $2.19+/-0.52$ & $0.010+/-0.002$ & $1.87+/-0.33$ & $0.008+/-0.001$ \\
\hline (R)SAL & $168.49+/-31.91$ & $0.94+/-0.18$ & $340.12+/-25.70$ & $1.90+/-0.14$ \\
\hline (S)SAL & $177.29+/-34.83$ & $0.99+/-0.19$ & $319.78+/-27.25$ & $1.78+/-0.15$ \\
\hline N-Me-(R)SAL & $193.02+/-21.32$ & $1.0+/-0.11$ & $82.36+/-13.54$ & $0.43+/-0.07$ \\
\hline $\mathrm{N}-\mathrm{Me}-(\mathrm{S}) \mathrm{SAL}$ & $181.43+/-32.54$ & $0.94+/-0.10$ & $102.54+/-18.70$ & $0.53+/-0.10$ \\
\hline NorSAL & $42.24+/-12.55$ & $0.26+/-0.08$ & $44.54+/-10.74$ & $0.27+/-0.07$ \\
\hline N-Me-NorSAL & $18.44+/-3.66$ & $0.10+/-0.02$ & $21.53+/-5.67$ & $0.12+/-0.03$ \\
\hline Dopamine & $987.74+/-120.55$ & $6.45+/-0.79$ & $503.38+/-72.71$ & $3.29+/-0.47$ \\
\hline DOPAC & $750.39+/-40.69$ * & $4.46+/-0.24$ * & $362.06+/-38.50$ * & $2.15+/-0.23$ * \\
\hline HVA & $833.00+/-63.12$ & $4.57+/-0.35$ & $399.41+/-57.25$ & $2.19+/-0.31$ \\
\hline
\end{tabular}


Table 3-2 (Continued).

\begin{tabular}{|c|c|c|c|c|}
\hline \multirow[t]{2}{*}{ Compound } & \multicolumn{2}{|c|}{ Normal Human Frontal Cortex } & \multicolumn{2}{|c|}{ PD Human Frontal Cortex } \\
\hline & $\mathrm{ng} / \mathrm{g}$ & $\mathrm{nM}$ & $\mathrm{ng} / \mathrm{g}$ & $\mathrm{nM}$ \\
\hline$\overline{T I Q}$ & $4.61+/-1.03$ & $0.035+/-0.008$ & $5.22+/-1.37$ & $0.04+/-0.01$ \\
\hline 1-Me-TIQ & $1.24+/-0.64$ & $0.008+/-0.004$ & $0.77+/-0.24$ & $0.005+/-0.002$ \\
\hline N-Me-TIQ & $1.03+/-0.33$ & $0.007+/-0.002$ & $0.61+/-0.13$ & $0.004+/-0.001$ \\
\hline 1-Benzyl-TIQ & $0.89+/-0.24$ & $0.004+/-0.001$ & $0.80+/-0.19$ & $0.004+/-0.001$ \\
\hline (R)SAL & $52.63+/-1.28$ & $0.294+/-0.007$ & $60.36+/-3.07$ & $0.34+/-0.02$ \\
\hline (S)SAL & $10.84+/-6.41$ & $0.060+/-0.04$ & $14.98+/-6.54$ & $0.08+/-0.04$ \\
\hline N-Me-(R)SAL & $71.30+/-2.36$ * & $0.37+/-0.01 *$ & $168.80+/-15.82$ * & $0.87+/-0.08$ * \\
\hline N-Me-(S)SAL & $66.75+/-4.77$ & $0.34+/-0.02$ & $135.43+/-22.05$ & $0.70+/-0.11$ \\
\hline NorSAL & $50.40+/-16.02$ & $0.31+/-0.10$ & $44.07+/-10.92$ & $0.27+/-0.07$ \\
\hline N-Me-NorSAL & $62.32+/-14.76$ & $0.35+/-0.08$ & $35.77+/-16.28$ & $1.97+/-0.09$ \\
\hline Dopamine & $4.77+/-1.63$ & $0.03+/-0.01$ & $2.60+/-0.12$ & $0.017+/-0.001$ \\
\hline DOPAC & $4.73+/-0.58$ & $0.028+/-0.003$ & $3.55+/-0.87$ & $0.021+/-0.005$ \\
\hline HVA & $3.86+/-1.53$ & $0.021+/-0.008$ & $3.53+/-0.85$ & $0.019+/-0.005$ \\
\hline \multirow{2}{*}{ Compound } & \multicolumn{2}{|c|}{ Normal Human Hippocampus } & \multicolumn{2}{|c|}{ PD Human Hippocampus } \\
\hline & $\mathrm{ng} / \mathrm{g}$ & $\mathrm{nM}$ & $\mathrm{ng} / \mathrm{g}$ & $\mathrm{nM}$ \\
\hline TIQ & $2.84+/-0.53$ & $0.021+/-0.004$ & $3.19+/-1.05$ & $0.024+/-0.008$ \\
\hline 1-Me-TIQ & $0.44+/-0.06$ & $0.0030+/-0.0004$ & $0.72+/-0.20$ & $0.005+/-0.001$ \\
\hline N-Me-TIQ & $0.69+/-0.10$ & $0.005+/-0.001$ & $1.04+/-0.15$ & $0.007+/-0.001$ \\
\hline 1-Benzyl-TIQ & $1.30+/-0.27$ & $0.006+/-0.001$ & $0.70+/-0.08$ & $0.0031+/-0.0004$ \\
\hline (R)SAL & $52.54+/-12.54$ * & $0.29+/-0.07^{*}$ & $182.73+/-12.73$ * & $1.02+/-0.07^{*}$ \\
\hline (S)SAL & $20.19+/-7.92$ * & $0.11+/-0.04$ * & $194.86+/-19.49$ * & $1.09+/-0.11$ * \\
\hline N-Me-(R)SAL & $125.90+/-20.50$ * & $0.65+/-0.11$ * & $612.65+/-27.23$ * & $3.17+/-0.14$ * \\
\hline N-Me-(S)SAL & $35.26+/-8.39$ * & $0.18+/-0.04$ * & $445.45+/-40.55$ * & $2.30+/-0.21$ * \\
\hline NorSAL & $70.94+/-4.21$ & $0.43+/-0.03$ & $68.79+/-3.79$ & $0.42+/-0.02$ \\
\hline N-Me-NorSAL & $39.02+/-7.82$ & $0.22+/-0.04$ & $46.78+/-5.95$ & $0.26+/-0.03$ \\
\hline Dopamine & $21.11+/-6.02$ & $0.14+/-0.04$ & $1.76+/-0.91$ & $0.011+/-0.006$ \\
\hline DOPAC & $16.20+/-3.07$ & $0.10+/-0.02$ & $1.51+/-0.81$ & $0.009+/-0.005$ \\
\hline HVA & $24.61+/-5.68$ & $0.13+/-0.03$ & $1.37+/-0.68$ & $0.008+/-0.004$ \\
\hline \multirow{2}{*}{ Compound } & \multicolumn{2}{|c|}{ Normal Human Inferior Olive } & \multicolumn{2}{|c|}{ PD Human Inferior Olive } \\
\hline & $\mathrm{ng} / \mathrm{g}$ & nM & $\mathrm{ng} / \mathrm{g}$ & nM \\
\hline TIQ & $1.95+/-0.26$ & $0.015+/-0.002$ & $2.31+/-0.80$ & $0.017+/-0.006$ \\
\hline 1-Me-TIQ & $0.53+/-0.18$ & $0.004+/-0.001$ & $0.79+/-0.21$ & $0.005+/-0.001$ \\
\hline N-Me-TIQ & $0.60+/-0.07$ & $0.0041+/-0.0005$ & $1.18+/-0.44$ & $0.008+/-0.003$ \\
\hline 1-Benzyl-TIQ & $1.52+/-0.44$ & $0.007+/-0.002$ & $0.91+/-0.31$ & $0.004+/-0.001$ \\
\hline (R)SAL & $23.78+/-2.10$ & $0.13+/-0.01$ & $14.61+/-5.64$ & $0.08+/-0.03$ \\
\hline (S)SAL & $24.05+/-4.58$ & $0.13+/-0.03$ & $8.27+/-3.37$ & $0.05+/-0.02$ \\
\hline N-Me-(R)SAL & $46.36+/-8.99$ & $0.24+/-0.05$ & $56.33+/-14.60$ & $0.29+/-0.08$ \\
\hline N-Me-(S)SAL & $16.77+/-4.21$ & $0.09+/-0.02$ & $31.75+/-8.12$ & $0.16+/-0.04$ \\
\hline NorSAL & $65.47+/-7.18$ & $0.40+/-0.04$ & $87.45+/-11.96$ & $0.54+/-0.07$ \\
\hline N-Me-NorSAL & $31.54+/-9.04$ & $0.18+/-0.05$ & $29.77+/-7.75$ & $0.17+/-0.04$ \\
\hline Dopamine & $8.54+/-2.10$ & $0.06+/-0.01$ & $7.56+/-2.15$ & $0.05+/-0.01$ \\
\hline DOPAC & $2.84+/-0.37$ & $0.017+/-0.002$ & $5.81+/-1.99$ & $0.03+/-0.01$ \\
\hline HVA & $4.70+/-0.79$ & $0.026+/-0.004$ & $6.33+/-1.11$ & $0.035+/-0.006$ \\
\hline \multirow{2}{*}{ Compound } & \multicolumn{2}{|c|}{ Normal Human Cerebellar Cortex } & \multicolumn{2}{|c|}{ PD Human Cerebellar Cortex } \\
\hline & $\mathrm{ng} / \mathrm{g}$ & $\mathrm{nM}$ & $\mathrm{ng} / \mathrm{g}$ & $\mathrm{nM}$ \\
\hline TIQ & $3.28+/-0.94$ & $0.025+/-0.007$ & $4.17+/-0.94$ & $0.031+/-0.007$ \\
\hline 1-Me-TIQ & $1.76+/-0.59$ & $0.012+/-0.004$ & $1.47+/-0.24$ & $0.010+/-0.002$ \\
\hline N-Me-TIQ & $1.34+/-0.23$ & $0.009+/-0.002$ & $1.63+/-0.36$ & $0.011+/-0.002$ \\
\hline 1-Benzyl-TIQ & $2.02+/-0.63$ & $0.009+/-0.003$ & $1.19+/-0.14$ & $0.005+/-0.001$ \\
\hline (R)SAL & $252.46+/-12.51$ & $1.41+/-0.07$ & $157.36+/-19.19$ & $0.88+/-0.11$ \\
\hline (S)SAL & $184.88+/-0.90$ & $1.032+/-0.005$ & $99.68+/-17.39$ & $0.56+/-0.10$ \\
\hline N-Me-(R)SAL & $60.85+/-6.63$ & $0.31+/-0.03$ & $33.45+/-6.01$ & $0.17+/-0.03$ \\
\hline N-Me-(S)SAL & $11.97+/-1.62$ & $0.062+/-0.008$ & $9.11+/-2.35$ & $0.05+/-0.01$ \\
\hline NorSAL & $112.62+/-9.47$ & $0.69+/-0.06$ & $78.12+/-10.11$ & $0.48+/-0.06$ \\
\hline N-Me-NorSAL & $28.15+/-7.35$ & $0.16+/-0.04$ & $23.76+/-4.31$ & $0.13+/-0.02$ \\
\hline Dopamine & $8.24+/-1.18$ & $0.054+/-0.008$ & $3.45+/-0.56$ & $0.023+/-0.004$ \\
\hline DOPAC & $4.41+/-0.72$ & $0.026+/-0.004$ & $1.67+/-0.43$ & $0.010+/-0.003$ \\
\hline HVA & $5.20+/-0.92$ & $0.029+/-0.005$ & $3.78+/-0.76$ & $0.021+/-0.004$ \\
\hline
\end{tabular}

Values are expressed as ng/g wet tissue weight +/- standard error.

* Significant difference between normal and PD. 
content in the mouse was also noted in the hippocampus, where $(R) S A L$ and DOPAC levels were greater than in the rat $(P<0.0008)$.

As in rodents, the levels of TIQ, 1-methyl-TIQ, N-methyl-TIQ and 1-benzyl-TIQ were similar across all regions in both normal human and PD brain, with an observed abundance of TIQ content over its derivatives. Importantly, several SAL derivatives were present at significantly lower concentrations in the caudate nucleus of PD patients in comparison with human controls (Table 3-2). Both $(R) S A L$ and $(S) S A L$, as well as $N$ methyl-(R)SAL and $N$-methyl-(S)SAL were noted at lower concentrations in this region of PD brain $(P<0.0006)$. The content of $N$-methyl- $(R) S A L$ in the substantia nigra of PD brain was also lower than that of normal human brain (Table 3-2; $P<0.0006$ ).

Conversely, $(R) S A L,(S) S A L$ and $N$-methyl-(S)SAL were present at significantly higher concentrations in the hippocampus of PD humans as compared to controls (Table 3-2; $P$ $<0.0006)$. Additionally, the concentration of $N$-methyl- $(R) S A L$ was significantly greater in the frontal cortex and hippocampus of PD brain $(P<0.0006)$. With the exception of DOPAC in the caudate nucleus, the concentrations of dopamine, DOPAC, and HVA were significantly lower in the nigrostriatal pathway (substantia nigra, caudate and putamen) of PD subjects in comparison with human controls (Table 3-2; $P<0.0005$ ).

A racemic mixture of the enantiomers of $(R / S) S A L$ and $N$-methyl- $(R / S) S A L$ was observed in regions of increased dopamine content, such as the striatum and ventral midbrain of rodents, and the caudate, putamen, substantia nigra and locus ceruleus of humans (Table 3-3). In the frontal cortices and cerebellae of humans and rodents, trends towards predominance of $(R)$ SAL over $(S) S A L$ did not attain statistical significance. However, similar trends towards predominance of $N$-methyl-(R)SAL over $N$-methyl-(S)SAL were significant in SD rats (frontal cortex), humans (cerebellar cortex) and C57BL/6 mice (cerebellum).

Regional methylation patterns were prominent in human brain (Table 3-4). For example, methylation of $(S) S A L$ was robust in human frontal cortex and minimal in cerebellar cortex. In catecholaminergic regions such as the substantia nigra, caudate, putamen and locus ceruleus, both enantiomers of SAL tended to exhibit higher concentrations than their methylated derivatives. In PD hippocampus, methylated derivatives of SAL were over two-fold more concentrated than their parent SAL. In contrast, this ratio was reversed in mouse and, to a lesser degree, in rat hippocampus.

Ratios of TIQ and TIQ derivatives to dopamine are presented in Table 3-5. In the hippocampus, all TIQ derivative/dopamine ratios were markedly higher in PD brain in comparison with normal human brain. Similar findings were noted in substantia nigra and were statistically significant for all of the salsolinols. For example, the $(R) \mathrm{SAL} /$ dopamine ratio was 14.9 times higher in PD brain (2.09) than in normal human brain (0.14). In the putamen and caudate, TIQ derivative/dopamine ratios also tended to show higher values in PD brain relative to tissues from the control brains. In contrast, frontal cortex, inferior olive and cerebellar cortex showed no differences in $\mathrm{TIQ}$ derivative/dopamine ratios between normal human and PD brain.

\subsubsection{Compound Stability}

Using ${ }^{1} \mathrm{H}$ NMR, all compounds under study were analyzed for stability after thermal $\left(22^{\circ} \mathrm{C}, 37^{\circ} \mathrm{C}\right.$ and $\left.60{ }^{\circ} \mathrm{C}\right)$ and freeze/thaw $\left(-80{ }^{\circ} \mathrm{C} / 22^{\circ} \mathrm{C}\right)$ exposure. All TIQ 
Table 3-3. Regional enantiomeric ratios.

\begin{tabular}{|c|c|c|c|c|}
\hline \multirow{2}{*}{ Brain Region } & \multicolumn{4}{|c|}{$(R)$ SAL / (S)SAL Ratio } \\
\hline & Normal Human & PD Human & SD Rat & C57BL/6 Mouse \\
\hline Frontal Cortex & $\begin{array}{c}2.18 \\
(P<0.0428)\end{array}$ & $\begin{array}{c}1.83 \\
(P<0.0173)\end{array}$ & $\begin{array}{c}2.23 \\
(P<0.0378)\end{array}$ & $\begin{array}{c}1.69 \\
(P<0.0443)\end{array}$ \\
\hline Striatum & - & - & $\begin{array}{c}1.14 \\
(P<0.3602)\end{array}$ & $\begin{array}{c}1.44 \\
(P<0.3091)\end{array}$ \\
\hline Caudate & $\begin{array}{c}1.01 \\
(P<0.9111)\end{array}$ & $\begin{array}{c}1.03 \\
(P<0.7139)\end{array}$ & r & ( \\
\hline Putamen & $\begin{array}{c}1.01 \\
(P<0.9275)\end{array}$ & $\begin{array}{c}1.12 \\
(P<0.1565)\end{array}$ & - & 19 \\
\hline Ventral Midbrain & - & - & $\begin{array}{c}1.12 \\
(P<0.1793)\end{array}$ & $\begin{array}{c}1.49 \\
(P<0.0806)\end{array}$ \\
\hline Substantia Nigra & $\begin{array}{c}1.05 \\
(P<0.8101)\end{array}$ & $\begin{array}{c}1.08 \\
(P<0.5574)\end{array}$ & ( & ( \\
\hline Hippocampus & $\begin{array}{c}1.60 \\
(P<0.0301)\end{array}$ & $\begin{array}{c}0.94 \\
(P<0.0942)\end{array}$ & $\begin{array}{c}1.66 \\
(P<0.0456)\end{array}$ & $\begin{array}{c}1.92 \\
(P<0.0186)\end{array}$ \\
\hline Locus Ceruleus & $\begin{array}{c}1.16 \\
(P<0.6481)\end{array}$ & $\begin{array}{c}1.09 \\
(P<0.2802)\end{array}$ & - & - \\
\hline Inferior Olive & $\begin{array}{c}1.07 \\
(P<0.6684)\end{array}$ & $\begin{array}{c}2.27 \\
(P<0.0350)\end{array}$ & - & - \\
\hline Cerebellum & - & ( & $\begin{array}{c}1.92 \\
(P<0.0401)\end{array}$ & $\begin{array}{c}1.60 \\
(P<0.0284)\end{array}$ \\
\hline Cerebellar Cortex & $\begin{array}{c}1.37 \\
(P<0.0293)\end{array}$ & $\begin{array}{c}1.35 \\
(P<0.0324)\end{array}$ & r & r \\
\hline \multirow{2}{*}{ Brain Region } & \multicolumn{4}{|c|}{ N-Methyl-(R)SAL / N-Methyl-(S)SAL Ratio } \\
\hline & Normal Human & PD Human & SD Rat & C57BL/6 Mouse \\
\hline Frontal Cortex & $\begin{array}{c}1.08 \\
(P<0.1098)\end{array}$ & $\begin{array}{c}1.37 \\
(P<0.1056)\end{array}$ & $\begin{array}{c}1.89^{*} \\
(P<0.0031)\end{array}$ & $\begin{array}{c}1.62 \\
(P<0.0557)\end{array}$ \\
\hline Striatum & - & 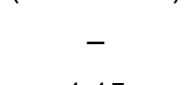 & $\begin{array}{c}1.25 \\
(P<0.1890)\end{array}$ & $\begin{array}{c}1.10 \\
(P<0.1818)\end{array}$ \\
\hline Caudate & $\begin{array}{c}0.98 \\
(P<0.8617)\end{array}$ & $\begin{array}{c}1.15 \\
(P<0.2457)\end{array}$ & - & - \\
\hline Putamen & $\begin{array}{c}1.01 \\
(P<0.8780)\end{array}$ & $\begin{array}{c}1.04 \\
(P<0.8423)\end{array}$ & - & - \\
\hline Ventral Midbrain & - & - & $\begin{array}{c}1.03 \\
(P<0.6505)\end{array}$ & $\begin{array}{c}1.10 \\
(P<0.1203)\end{array}$ \\
\hline Substantia Nigra & $\begin{array}{c}0.94 \\
(P<0.0760)\end{array}$ & $\begin{array}{c}1.10 \\
(P<0.5649)\end{array}$ & - & ( \\
\hline Hippocampus & $\begin{array}{c}4.02 \\
(P<0.0128)\end{array}$ & $\begin{array}{c}1.41 \\
(P<0.0802)\end{array}$ & $\begin{array}{c}1.38^{*} \\
(P<0.0120)\end{array}$ & $\begin{array}{c}2.93^{*} \\
(P<0.0097)\end{array}$ \\
\hline Locus Ceruleus & $\begin{array}{c}1.13 \\
(P<0.3017)\end{array}$ & $\begin{array}{c}0.80^{*} \\
(P<0.0059)\end{array}$ & - & - \\
\hline Inferior Olive & $\begin{array}{c}3.18 \\
(P<0.0343)\end{array}$ & $\begin{array}{c}1.71 \\
(P<0.0126)\end{array}$ & - & - \\
\hline Cerebellum & - & - & $\begin{array}{c}1.59 \\
(P<0.0185)\end{array}$ & $\begin{array}{c}4.39^{*} \\
(P<0.0073)\end{array}$ \\
\hline Cerebellar Cortex & $\begin{array}{c}4.97^{*} \\
(P<0.0005) \\
\end{array}$ & $\begin{array}{c}4.24^{*} \\
(P<0.0060)\end{array}$ & - & - \\
\hline
\end{tabular}

* Significantly different from 1. 
Table 3-4. Regional methylation ratios.

\begin{tabular}{|c|c|c|c|c|}
\hline \multirow{2}{*}{ Brain Region } & \multicolumn{4}{|c|}{$N$-methyl-(R)SAL / $(R)$ SAL Ratio } \\
\hline & Normal Human & PD Human & SD Rat & C57BL/6 Mouse \\
\hline Frontal Cortex & $\begin{array}{c}1.35 \\
(P<0.0180)\end{array}$ & $\begin{array}{c}2.90^{*} \\
(P<0.0061)\end{array}$ & $\begin{array}{c}3.07^{*} \\
(P<0.0008)\end{array}$ & $\begin{array}{c}0.71 \\
(P<0.4444)\end{array}$ \\
\hline Striatum & - & - & $\begin{array}{c}0.76 \\
(P<0.1171)\end{array}$ & $\begin{array}{c}0.90 \\
(P<0.5672)\end{array}$ \\
\hline Caudate & $\begin{array}{c}0.70 \\
(P<0.0582)\end{array}$ & $\begin{array}{c}0.37^{*} \\
(P<0.0009)\end{array}$ & ( & ( \\
\hline Putamen & $\begin{array}{c}0.66 \\
(P<0.0165)\end{array}$ & $\begin{array}{c}0.92 \\
(P<0.4969)\end{array}$ & 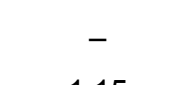 & 10 \\
\hline Ventral Midbrain & - & - & $\begin{array}{c}1.15 \\
(P<0.1436)\end{array}$ & $\begin{array}{c}1.48 \\
(P<0.0231)\end{array}$ \\
\hline Substantia Nigra & $\begin{array}{c}0.89 \\
(P<0.4236)\end{array}$ & $\begin{array}{c}0.29^{*} \\
(P<0.0005)\end{array}$ & ( & ( \\
\hline Hippocampus & $\begin{array}{c}4.06 \\
(P<0.2324)\end{array}$ & $\begin{array}{c}3.41^{*} \\
(P<0.0009)\end{array}$ & $\begin{array}{c}0.72 \\
(P<0.0803)\end{array}$ & $\begin{array}{c}0.26^{*} \\
(P<0.0005)\end{array}$ \\
\hline Locus Ceruleus & $\begin{array}{c}1.37 \\
(P<0.3013)\end{array}$ & $\begin{array}{c}0.24^{*} \\
(P<0.0005)\end{array}$ & - & - \\
\hline Inferior Olive & $\begin{array}{c}1.94 \\
(P<0.0618)\end{array}$ & $\begin{array}{c}4.12 \\
(P<0.1261)\end{array}$ & - & - \\
\hline Cerebellum & - & - & $\begin{array}{c}1.28 \\
(P<0.3684)\end{array}$ & $\begin{array}{c}1.08 \\
(P<0.7712)\end{array}$ \\
\hline Cerebellar Cortex & $\begin{array}{c}0.24^{*} \\
(P<0.0005)\end{array}$ & $\begin{array}{c}0.22^{*} \\
(P<0.0005)\end{array}$ & r & r \\
\hline \multirow{2}{*}{ Brain Region } & \multicolumn{4}{|c|}{ N-methyl-(S)SAL / (S)SAL Ratio } \\
\hline & Normal Human & PD Human & SD Rat & C57BL/6 Mouse \\
\hline Frontal Cortex & $\begin{array}{c}2.73^{*} \\
(P<0.0009)\end{array}$ & $\begin{array}{c}4.18^{*} \\
(P<0.0005)\end{array}$ & $\begin{array}{c}6.33^{*} \\
(P<0.0005)\end{array}$ & $\begin{array}{c}0.70 \\
(P<0.3823)\end{array}$ \\
\hline Striatum & - & 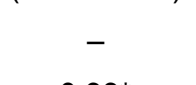 & $\begin{array}{c}0.70 \\
(P<0.0821)\end{array}$ & $\begin{array}{c}1.33 \\
(P<0.6129)\end{array}$ \\
\hline Caudate & $\begin{array}{c}0.71^{*} \\
(P<0.0013)\end{array}$ & $\begin{array}{c}0.33^{*} \\
(P<0.0005)\end{array}$ & - & 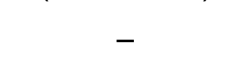 \\
\hline Putamen & $\begin{array}{c}0.65^{\star} \\
(P<0.0010)\end{array}$ & $\begin{array}{c}1.05 \\
(P<0.6867)\end{array}$ & - & - \\
\hline Ventral Midbrain & - & - & $\begin{array}{c}1.25 \\
(P<0.0362)\end{array}$ & $\begin{array}{c}1.98 \\
(P<0.0197)\end{array}$ \\
\hline Substantia Nigra & $\begin{array}{c}0.91 \\
(P<0.5284)\end{array}$ & $\begin{array}{c}0.27^{*} \\
(P<0.0005)\end{array}$ & - & - \\
\hline Hippocampus & $\begin{array}{c}1.78 \\
(P<0.4818)\end{array}$ & $\begin{array}{c}2.34^{*} \\
(P<0.0071)\end{array}$ & $\begin{array}{c}0.80 \\
(P<0.0963)\end{array}$ & $\begin{array}{c}0.17^{*} \\
(P<0.0005)\end{array}$ \\
\hline Locus Ceruleus & $\begin{array}{c}1.12 \\
(P<0.6173)\end{array}$ & $\begin{array}{c}0.33^{*} \\
(P<0.0005)\end{array}$ & - & - \\
\hline Inferior Olive & $\begin{array}{c}0.72 \\
(P<0.2769)\end{array}$ & $\begin{array}{c}5.09^{*} \\
(P<0.0005)\end{array}$ & - & - \\
\hline Cerebellum & - & - & $\begin{array}{c}1.64 \\
(P<0.2748)\end{array}$ & $\begin{array}{c}0.34^{*} \\
(P<0.0005)\end{array}$ \\
\hline Cerebellar Cortex & $\begin{array}{c}0.07^{*} \\
(P<0.0005)\end{array}$ & $\begin{array}{c}0.08^{*} \\
(P<0.0005)\end{array}$ & - & - \\
\hline
\end{tabular}

* Significantly different from 1. 
Table 3-5. Regional TIQ derivative/dopamine ratios in human brain.

\begin{tabular}{|c|c|c|c|c|c|c|}
\hline \multirow{2}{*}{ Compound Ratio } & \multicolumn{3}{|c|}{ Caudate } & \multicolumn{3}{|c|}{ Putamen } \\
\hline & Normal Human & PD Human & $P$ Value & Normal Human & PD Human & $P$ Value \\
\hline TIQ / Dopamine & 0.00041 & 0.0029 & 0.0431 & 0.00088 & 0.0026 & 0.0141 \\
\hline 1-methyl-TIQ / Dopamine & 0.00019 & 0.0015 & $0.0002^{*}$ & 0.00049 & 0.00093 & 0.0224 \\
\hline N-methyl-TIQ / Dopamine & 0.00012 & 0.0010 & 0.0141 & 0.00045 & 0.00084 & 0.0996 \\
\hline 1-benzyl-TIQ / Dopamine & 0.00020 & 0.0014 & $0.0011^{*}$ & 0.00034 & 0.00043 & 0.5218 \\
\hline (R)SAL / Dopamine & 0.057 & 0.20 & 0.0082 & 0.14 & 0.23 & $0.0037^{*}$ \\
\hline (S)SAL / Dopamine & 0.11 & 0.20 & 0.1685 & 0.51 & 0.21 & 0.0665 \\
\hline$N$-methyl-(R)SAL / Dopamine & 0.039 & 0.068 & 0.0180 & 0.093 & 0.21 & $0.0010^{*}$ \\
\hline N-methyl-(S)SAL / Dopamine & 0.040 & 0.062 & 0.0796 & 0.092 & 0.22 & 0.0087 \\
\hline NorSAL / Dopamine & 0.0096 & 0.040 & $0.0007^{*}$ & 0.026 & 0.030 & 0.3751 \\
\hline \multirow{2}{*}{ Compound Ratio } & \multicolumn{3}{|c|}{ Substantia Nigra } & \multicolumn{3}{|c|}{ Locus Ceruleus } \\
\hline & Normal Human & PD Human & $P$ Value & Normal Human & PD Human & $P$ Value \\
\hline TIQ / Dopamine & 0.0030 & 0.035 & 0.0580 & 0.0051 & 0.010 & 0.0322 \\
\hline 1-methyl-TIQ / Dopamine & 0.0011 & 0.016 & 0.0583 & 0.0021 & 0.0037 & 0.1415 \\
\hline N-methyl-TIQ / Dopamine & 0.0015 & 0.015 & 0.0456 & 0.0019 & 0.0032 & 0.1816 \\
\hline 1-benzyl-TIQ / Dopamine & 0.00096 & 0.0073 & 0.0062 & 0.0021 & 0.0036 & 0.0522 \\
\hline (R)SAL / Dopamine & 0.14 & 2.09 & $0.0027^{*}$ & 0.19 & 0.75 & 0.0081 \\
\hline (S)SAL / Dopamine & 0.17 & 1.98 & $0.0034^{*}$ & 0.19 & 0.69 & 0.0094 \\
\hline$N$-methyl-(R)SAL / Dopamine & 0.12 & 0.54 & $<0.0001^{*}$ & 0.20 & 0.17 & 0.3874 \\
\hline N-methyl-(S)SAL / Dopamine & 0.13 & 0.50 & $<0.0001^{*}$ & 0.18 & 0.21 & 0.4979 \\
\hline NorSAL / Dopamine & 0.043 & 0.46 & $0.0022^{*}$ & 0.039 & 0.085 & 0.0139 \\
\hline \multirow{2}{*}{ Compound Ratio } & \multicolumn{3}{|c|}{ Frontal Cortex } & \multicolumn{3}{|c|}{ Hippocampus } \\
\hline & Normal Human & PD Human & $P$ Value & Normal Human & PD Human & $P$ Value \\
\hline TIQ / Dopamine & 1.82 & 1.99 & 0.8534 & 0.18 & 4.65 & $0.0015^{\star}$ \\
\hline 1-methyl-TIQ / Dopamine & 0.42 & 0.28 & 0.6216 & 0.04 & 0.77 & $0.0017^{*}$ \\
\hline N-methyl-TIQ / Dopamine & 0.66 & 0.22 & 0.3393 & 0.04 & 1.75 & $0.0045^{*}$ \\
\hline 1-benzyl-TIQ / Dopamine & 0.29 & 0.31 & 0.8523 & 0.07 & 1.42 & $0.0035^{\star}$ \\
\hline (R)SAL / Dopamine & 34.23 & 23.37 & 0.6612 & 5.16 & 390.17 & $<0.0001^{*}$ \\
\hline (S)SAL / Dopamine & 4.24 & 6.55 & 0.5899 & 1.39 & 408.16 & $<0.0001^{*}$ \\
\hline N-methyl-(R)SAL / Dopamine & 43.82 & 66.21 & 0.4733 & 6.76 & 1253.31 & $<0.0001^{*}$ \\
\hline N-methyl-(S)SAL / Dopamine & 38.16 & 53.02 & 0.0714 & 1.77 & 827.47 & $<0.0001^{*}$ \\
\hline NorSAL / Dopamine & 12.92 & 16.92 & 0.5243 & 5.35 & 136.82 & $0.0007^{*}$ \\
\hline \multirow{2}{*}{ Compound Ratio } & \multicolumn{3}{|c|}{ Inferior Olive } & \multicolumn{3}{|c|}{ Cerebellar Cortex } \\
\hline & Normal Human & PD Human & $P$ Value & Normal Human & PD Human & $P$ Value \\
\hline TIQ / Dopamine & 0.27 & 0.29 & 0.8556 & 0.40 & 1.26 & 0.0087 \\
\hline 1-methyl-TIQ / Dopamine & 0.07 & 0.13 & 0.1820 & 0.20 & 0.44 & 0.0497 \\
\hline N-methyl-TIQ / Dopamine & 0.09 & 0.18 & 0.2977 & 0.17 & 0.45 & 0.0060 \\
\hline 1-benzyl-TIQ / Dopamine & 0.20 & 0.21 & 0.9294 & 0.21 & 0.39 & 0.0923 \\
\hline (R)SAL / Dopamine & 3.22 & 2.07 & 0.1523 & 35.01 & 54.47 & 0.3300 \\
\hline (S)SAL / Dopamine & 3.86 & 1.52 & 0.1204 & 28.17 & 28.98 & 0.9031 \\
\hline$N$-methyl-(R)SAL / Dopamine & 6.04 & 8.29 & 0.2732 & 7.90 & 9.55 & 0.2104 \\
\hline N-methyl-(S)SAL / Dopamine & 1.87 & 5.00 & 0.0099 & 1.60 & 2.44 & 0.1007 \\
\hline NorSAL / Dopamine & 9.10 & 22.75 & 0.0145 & 14.94 & 24.93 & 0.0129 \\
\hline
\end{tabular}

* Significant difference between normal and PD. 
derivatives were resistant to any thermal-induced structural alterations, demonstrating the exceptional stability of these aromatic organic compounds. For illustration, examination and comparison of NMR spectra for TIQ at baseline (Appendix A, Figure A1A) and after dry heat exposure at $60{ }^{\circ} \mathrm{C}$ for 28 days (Appendix A, Figure A-1B) revealed excellent stability in aqueous solution; no additional peaks or peak shifts were noted. NMR spectra for all other study compounds are provided in Appendix A.

\subsection{DISCUSSION}

Consistent with the data presented herein, other investigators have detected select TIQ derivatives in rodent and human brain (Deng et al 1997; Kohno et al 1986; Kotake et al 1995; Makino et al 1988; Maruyama et al 1997c; Musshoff et al 2003; Musshoff et al 2005; Musshoff et al 2000; Musshoff et al 1999; Niwa et al 1987; Niwa et al 1991; Niwa et al 1989; Sasaoka et al 1988; Sjoquist et al 1982a; b; Tasaki et al 1991). Collectively, however, earlier studies were largely limited to individual compounds without adequate consideration of regional distribution, enantiomers or species differences. In contrast, our work presents a comprehensive regional and quantitative picture of TIQ derivatives in mouse, rat, normal human and PD brain. Although TIQ derivatives were detected in all brain regions analyzed across all species, $(R / S) S A L$ and its methylated derivatives were detected at higher levels in regions of increased dopamine content in both rodents and humans. Dopaminergic regions also revealed a racemic mixture of $(R)$ - and $(S)$-enantiomers. Of greatest importance, the concentrations of $(R / S) S A L$ and $N$-methyl- $(R / S) S A L$ were significantly lower in the caudate and, to a lesser degree, in the substantia nigra of PD brains in comparison with normal human controls. In addition, the results of our stability experiments show that TIQ derivatives are not short-lived, unstable intermediates and, conceivably, could accumulate in catecholaminergic neuronal populations with aging.

Previous results derived from homogenates of rodent whole brain are quantitatively similar to our own findings. For instance, with gas chromatography-mass spectroscopy (GC-MS), Makino and colleagues detected TIQ in rat whole brain at a concentration of $4.2 \mathrm{ng} / \mathrm{g}$ (Makino et al 1988). In similar fashion, Tasaki and colleagues quantified TIQ and 1-methyl-TIQ in mouse whole brain homogenate at concentrations of $1.1 \mathrm{ng} / \mathrm{g}$ and $9.8 \mathrm{ng} / \mathrm{g}$, respectively (Tasaki et al 1991). Tasaki et al. also found 1methyl-TIQ in rat whole brain at a concentration of $1.7 \mathrm{ng} / \mathrm{g}$. Using GC-MS, Kohno and colleagues detected TIQ at concentrations ranging from 5 to $7 \mathrm{ng} / \mathrm{g}$, and 1-methyl-TIQ at concentrations ranging from 1 to $3 \mathrm{ng} / \mathrm{g}$ in untreated rat brain (Kohno et al 1986). More recently, Kotake and colleagues quantified 1-benzyl-TIQ in mouse brain at a concentration of $7.67 \mathrm{ng} / \mathrm{g}$ (Kotake et al 1995). Our results in mice and rats indicate that TIQ, 1-methyl-TIQ, N-methyl TIQ and 1-benzyl-TIQ are widely distributed in brain with minimal regional variation in concentrations. Since TIQ, 1-methyl-TIQ, N-methyl TIQ and 1-benzyl-TIQ are not concentrated in catecholaminergic pathways, it is possible that they arise from exogenous sources since all of these compounds readily cross the blood-brain barrier (Song et al 2006). Alternatively, these compounds may arise from endogenous synthetic pathways that do not require catecholamines.

In contrast to TIQ, 1-methyl-TIQ, N-methyl TIQ and 1-benzyl-TIQ, regional quantification of $(R) S A L,(S) S A L$, and their methylated derivatives in rodent brain points out an important role for endogenous synthetic pathways. Overall, our results demonstrate elevated concentrations of $(R) S A L,(S) S A L$ and their methylated derivatives 
in the nigrostriatal pathway of rodent brain. Furthermore, generalized trends towards predominance of the $(R)$-enantiomer of SAL and its methylated derivative were noted in cerebral cortex, cerebellum and hippocampus. As depicted in Figure 3-2, $(R)$ enantiomeric excess could be the manifestation of stereoselective enzymatic synthesis via salsolinol synthase, coupled with low-level racemic Pictet-Spengler condensation, in areas with lower overall dopamine concentrations. In areas with higher dopamine content, negligible enantiomeric excess was observed, suggesting a greater contribution from the Pictet-Spengler reaction. These interpretations are supported by recent evidence of a dose-dependent increase in striatal $(R / S) S A L$ levels and a concomitant decrease in striatal dopamine content upon intracerebral infusion of acetaldehyde in freely-moving rats (Wang et al 2007).

Species differences in the regional concentrations of TIQ derivatives among rodents were most prominent in the striatum where the concentrations of $N$-methyl$(R) S A L$ and $N$-methyl-(S)SAL were much higher in mice than rats despite the absence of significant differences in either dopamine or $(R / S) S A L$ content in this region. The concentrations of ventral midbrain NorSAL and hippocampal $(R) S A L$ were also elevated in the mouse as compared to the rat. In contrast, the concentrations of dopamine and $N$-methyl-(S)SAL were higher in the ventral midbrains from rats compared to mice. Although this mixture of observations could be attributed to a multitude of factors, their magnitude suggests the presence of genetic contributions to endogenous synthesis and cellular metabolism of these compounds.

In humans, previous studies have focused on SAL and SAL derivatives in the context of alcoholism and, to a lesser degree, PD. Comparatively little effort has been placed on TIQ and its methylated and benzylated derivatives. Using GC-MS, TIQ, 1methyl-TIQ and N-methyl-TIQ have been detected in normal human and PD brain. In a single PD case, TIQ was quantified in the frontal cortex at $10 \mathrm{ng} / \mathrm{g}$ (Niwa et al 1987). Analysis of frontal cortex from a single normal human brain detected TIQ at less than 1 $\mathrm{ng} / \mathrm{g}$. This same study also quantified $N$-methyl-TIQ in frontal cortex at $2 \mathrm{ng} / \mathrm{g}$ in PD brain and $3 \mathrm{ng} / \mathrm{g}$ in control brain whereas 1 -methyl-TIQ was reportedly undetectable in both normal human and PD brain. In a subsequent publication from the same group, TIQ was detected in the occipital cortex of a single PD brain at $7 \mathrm{ng} / \mathrm{g}$ (Niwa et al 1989). As was the case for rodents, we found that TIQ, 1-methyl-TIQ, N-methyl-TIQ and 1benzyl-TIQ are widely distributed in brain with no significant concentration differences among regions or between normal human and PD brain.

The discovery that $(R / S) S A L$ can be synthesized from acetaldehyde and dopamine under physiological conditions in both plants and animals (Schöpf \& Bayerle 1934) stimulated a great deal of speculation and research regarding the potential role of SAL derivates in human diseases. Seminal GC-MS studies by Sjoquist and colleagues documented $(R / S) S A L$ and $N$-methyl-( $R / S) S A L$ in postmortem caudate (5 to $23 \mathrm{ng} / \mathrm{g}$ and 5 to $10 \mathrm{ng} / \mathrm{g}$, respectively), putamen ( 9 to $35 \mathrm{ng} / \mathrm{g}$ and 6 to $12 \mathrm{ng} / \mathrm{g}$, respectively), hippocampus (13 to $35 \mathrm{ng} / \mathrm{g}$ and 2 to $5 \mathrm{ng} / \mathrm{g}$, respectively), and frontal cortex (1 to 32 $\mathrm{ng} / \mathrm{g}$ and 2 to $5 \mathrm{ng} / \mathrm{g}$, respectively) in alcoholic $(N=5)$ and control $(N=5)$ human brain, and proposed a positive correlation between endogenous SAL formation and regional dopamine content (Sjoquist et al 1982a; b). In subsequent work, liquid chromatography was employed for the detection of $(R / S) S A L$ in the caudate $(39 \mathrm{ng} / \mathrm{g})$ and putamen $(37$ $\mathrm{ng} / \mathrm{g}$ ) of a single normal human brain (Sasaoka et al 1988). Semi-quantitative GC-MS analysis of $N$-methyl- $(R / S) S A L$ in PD $(N=6)$ and normal human $(N=5)$ frontal cortex found an "estimated" concentration of $1 \mathrm{ng} / \mathrm{g}$ for both groups (Niwa et al 1991). More 
recently, Deng and colleagues used enantiomeric-selective HPLC to quantify $(R) S A L$ and $(S) S A L$, as well as $N$-methyl-(R)SAL and $N$-methyl-( $S) S A L$ in "gray matter" from a single normal human brain (Deng et al 1997). While this analysis failed to detect either (S)SAL or $N$-methyl-(S)SAL, $(R) S A L$ and $N$-methyl-(R)SAL were quantified at $12 \mathrm{ng} / \mathrm{g}$ and $17 \mathrm{ng} / \mathrm{g}$, respectively. In similar fashion, Maruyama and colleagues quantified $(R) S A L,(S) S A L, N$-methyl-(R)SAL and $N$-methyl-(S)SAL in the fronal cortex, caudate, putamen and substantia nigra of normal human brain $(N=10)$ (Maruyama et al 1997c). Once again, $(S)$-enantiomers were not detected, while concentrations of $(R) S A L$ ranged from $6 \mathrm{ng} / \mathrm{g}$ in the putamen to $24 \mathrm{ng} / \mathrm{g}$ in the frontal cortex. The content of $\mathrm{N}$-methyl$(R) S A L$ ranged from $1 \mathrm{ng} / \mathrm{g}$ in the frontal cortex to $20 \mathrm{ng} / \mathrm{g}$ in the putamen. These results should be interpreted with caution, however, since complete absence of the (S)enantiomers of SAL and $N$-methyl-SAL in brain is not compatible with our own findings and unlikely based upon the (1) presence of $(S) S A L$ in foods, (2) non-enzymatic, racemic SAL synthesis via the Pictet-Spengler condensation reaction, and (3) studies by Musshoff and co-workers outlined below (Cohen \& Collins 1970; Deng et al 1997; Musshoff et al 2003; Musshoff et al 2005; Musshoff et al 2000; Musshoff et al 1999; Schöpf \& Bayerle 1934).

From the late 1990s to 2005, Musshoff and colleagues established a large collection of postmortem alcoholic $(N=44)$ and control $(N=47)$ human brains (Musshoff et al 2003; Musshoff et al 2005; Musshoff et al 2000; Musshoff et al 1999). Using SPE and GC-MS, $(R) S A L,(S) S A L$ and NorSAL content was determined in several regions of the brain. $(R) S A L$ was detected in the caudate nuclei of both alcoholics and controls (17 $\mathrm{ng} / \mathrm{g}$ and $21 \mathrm{ng} / \mathrm{g}$, respectively), as was (S)SAL ( $5 \mathrm{ng} / \mathrm{g}$ and $5 \mathrm{ng} / \mathrm{g}$, respectively) and NorSAL (12 ng/g and $40 \mathrm{ng} / \mathrm{g}$, respectively). In the putamen, the content of $(R) S A L$ in alcoholics and controls was $13 \mathrm{ng} / \mathrm{g}$ and $25 \mathrm{ng} / \mathrm{g}$, respectively; (S)SAL was quantified at $5 \mathrm{ng} / \mathrm{g}$ for both groups; NorSAL was detected at $16 \mathrm{ng} / \mathrm{g}$ and $30 \mathrm{ng} / \mathrm{g}$, respectively. In the substantia nigra, the content of $(R) S A L$ in control subjects was $29 \mathrm{ng} / \mathrm{g}$; $(S) S A L$ was quantified at $19 \mathrm{ng} / \mathrm{g}$; NorSAL was detected at $12 \mathrm{ng} / \mathrm{g}$. No data from the substantia nigra of alcoholic subjects was presented. Furthermore, $(R) S A L,(S) S A L$ and NorSAL were not detected in the hippocampus, frontal cortex or cerebellum.

As is readily apparent, considerable variability and discrepancies are present among previous studies of regional SAL derivative content in PD, alcoholic and normal human brain. In general, however, our findings are qualitatively similar to those of Musshoff and co-workers (Musshoff et al 2003; Musshoff et al 2005; Musshoff et al 2000; Musshoff et al 1999) although the absolute concentrations for brain $(R) S A L$, (S)SAL and their derivatives determined with HPLC-EC are somewhat higher than previous reports generated with GC-MS. These concentration differences may be attributed to one or more factors. First of all, our control and PD subjects were approximately 70 years of age whereas Musshoff and colleagues used postmortem tissue from much younger subjects (e.g., 46.5 years for alcoholics and 37.0 years for controls). Second, the postmortem interval was less than $6 \mathrm{hrs}$ in our study but was extended to $144 \mathrm{hrs}$ in the studies by Musshoff and colleagues. Lastly, most studies of SAL and SAL derivatives in human brain utilized GC-MS rather than HPLC-EC. Relative to HPLC-EC, GC-MS is associated with reduced compound retrieval since sample preparation requires multiple organic extractions and compound derivatization prior to analysis. For example, in 47 control subjects, Musshoff's group reported mean putamen dopamine concentration as $1305 \mathrm{ng} / \mathrm{g}$. In contrast, Konradi and colleagues reported mean putamen dopamine concentrations of 3000 to $7000 \mathrm{ng} / \mathrm{g}$ using HPLC analysis in a cohort of 24 normal human subjects (Konradi et al 1992). Our own extraction protocol, 
quantification methods and results (mean putamen dopamine concentration of 4142 $\mathrm{ng} / \mathrm{g}$ ) are similar to those of Konradi et al. (Konradi et al 1992). Thus, it is clear that tissue preparation and analyte extraction for GC-MS is much less efficient than the straightforward HPLC extraction procedures used in our study and the comparison of dopamine concentration can be extrapolated to analysis of TIQ derivatives. As in rodents, $(R / S) S A L$ and $N$-methyl-(R/S)SAL concentrations were highest in dopaminergic regions (caudate, putamen, substantia nigra) of both normal and PD human brain. Also similar to rodents, general trends towards enantiomeric excess of $(R) S A L$ and $N$-methyl$(R) S A L$ were found in cerebral cortex, cerebellar cortex and hippocampus.

In comparison to human controls, the concentrations of (R)SAL, $(S) S A L, N$ methyl- $(R) S A L$ and $N$-methyl-(S)SAL were significantly decreased in PD caudate and the concentration of $N$-methyl-(R)SAL was significantly decreased in PD substantia nigra. With correction for multiple comparisons, the mean differences in SAL and SAL derivative concentrations between PD and control brain in "catecholamingeric regions" of the brain (substantia nigra, caudate, putamen and locus ceruleus) did not reach statistical significance. Among those compounds showing concentration differences between control and PD brain, $N$-methyl- $(R) S A L$ deserves particular attention. Although the neurotoxicity of 1-benzyl-TIQ (Shavali \& Ebadi 2003) has been clearly documented and $(R / S) S A L$, NorSAL and $N$-methyl-NorSAL are associated with apoptosis in vitro (Maruyama et al 1997a; Storch et al 2000), current data suggests that $N$-methyl-(R)SAL may be the most potent TIQ neurotoxin (Naoi et al 2002). $N$-methyl-(R)SAL has exhibited marked toxicity in cultured rat and human dopaminergic neurons (Akao et al 1999; Maruyama et al 2001; Naoi et al 2000; Naoi et al 1997), inducing apoptosis in $100 \%$ of dopaminergic human neuroblastoma SH-SY5Y cells after exposure (Maruyama et al 2001; Maruyama et al 1997a). In the same study, (R/S)SAL and N-methyl-(S)SAL induced apoptotic cell death in only $10 \%$ and $11 \%$ of SH-SY5Y cells, respectively. When coupled with previous toxicity studies, our findings suggest that $(R / S) S A L$ and $N$ methyl-(R/S)SAL are derived from dopamine and concentrated within dopaminergic neurons. At some critical concentration, these TIQ derivatives may contribute to the apoptotic cell death of dopaminergic neurons in PD and are released into the interstitial fluid. In support of this concept, Moser and Kömpf detected both $(R / S) S A L$ and $N$ methyl-NorSAL in the lumbar cerebrospinal fluid of PD patients (Moser \& Kompf 1992). Furthermore, the CSF content of $N$-methyl-(R)SAL is higher in newly diagnosed, untreated PD patients compared to normal human controls (Maruyama et al 1999).

In PD, the most prominent neurodegenerative cell death is seen in the catecholaminergic neurons of the SNpc (dopaminergic) and locus ceruleus (noradrenergic). Accordingly, the most straightforward explanation for the relatively selective cell death seen in PD is the accumulation of neurotoxins derived from catecholamines. TIQs have been shown to inhibit complex I of the respiratory chain (McNaught et al 1995b) and are one class of neurotoxins that can be derived from catecholamines. Several TIQs can cross the blood-brain barrier and, in the peripheral sympathetic nervous system, certain TIQs are taken up by the norepinephrine transporter. Thus, TIQs may accumulate in dopaminergic and noradrenergic systems with aging via a combination of exogenous and endogenous pathways. Furthermore, in comparison with normal human brain, there may be greater production, accumulation or persistence of TIQ derivatives, particularly the salsolinols, in PD brain. Aggregate accumulation of TIQs and other neurotoxic derivatives of dopamine and norepinephrine may reach critical levels in aged individuals and contribute to catecholaminergic cell death in PD. 
Using ${ }^{1} \mathrm{H}$ NMR spectroscopy, all TIQ derivatives utilized in this study were fully stable at $60{ }^{\circ} \mathrm{C}$ for 28 days in aqueous solution. This finding is most readily attributable to the aromaticity and general planar structure of the TIQ family of compounds (Martin \& Gudzinowicz 1963). In the context of PD, the relatively high thermal stability of TIQ derivatives permits consideration of environmental sources, such as foods and well water.

Catecholamines, such as dopamine and norepinephrine, are typically metabolized in the brain via the enzymatic action of MAO and catechol-O-methyl transferase (COMT). Likewise, several studies describe the COMT mono-O-methylation pattern of SAL in intact rat brain and suggest that delayed $O$-methylation in dopaminergic regions may be a result of active uptake and storage mechanisms in these areas (Bail et al 1980; Collins \& Origitano 1983; Origitano \& Collins 1980). Furthermore, similar methylation patterns from multiple brain regions suggest that a ubiquitous COMT enzyme is responsible for catechol TIQ derivative metabolism (Collins \& Origitano 1983). In contrast, however, TIQ and many of its derivatives (1-methyl-TIQ, N-methyl-TIQ, $(R / S) S A L, N$-methyl-(R/S)SAL and $N$-methyl-NorSAL) do not appear to be substrates for either MAO-A- or MAO-B (Bembenek et al 1990). Instead, isoquinolines and TIQs show selective inhibition of either MAO-A or MAO-B (Bembenek et al 1990; Collins et al 1973; Dostert et al 1989; Meyerson et al 1976; Minami et al 1993; Thull et al 1995; Yamada 1971). Based on the high thermal stability of TIQ derivatives, it is possible that the major pathway for TIQ metabolism in vivo is enzymatic via COMT. It should be noted, however, that other catecholamine metabolizing enzymes, such as the phenol sulfotransferases (Axelrod et al 1959; Levy et al 1975; Pacifici et al 1994; Richter 1940; Young et al 1984), and non-enzymatic pathways may contribute the metabolism/degradation of TIQ derivatives.

The widespread pathology in PD was first emphasized in seminal work published by Den Hartog Jager and Bethlem in 1960. In their study, Lewy bodies, the pathological hallmark of PD, were detected in the sympathetic ganglia in five of six PD cases (Den Hartog Jager \& Bethlem 1960). In the CNS, there were Lewy bodies in the SNpc, locus ceruleus, hypothalamus and several brainstem nuclei. The marked topological prevalence of Lewy bodies for catecholaminergic cell groups is compatible with the hypotheses that the accumulation of neurotoxic by-products of catecholamines, such at salsolinols, may play a role in the etiopathogenesis of PD.

\subsection{CONCLUSIONS}

Several members of the TIQ family of monoamine alkaloids can be formed from dopamine or its oxidized metabolites and may be involved in the pathogenesis of monoaminergic cell death in PD. Using enantiomeric-selective HPLC-EC and LCMS/MS, the regional concentrations of several TIQ derivatives, including salsolinols, were determined in mouse, rat, normal human and PD brain. TIQ derivatives were detected in all regions subjected to analysis. In general, salsolinols were present at higher concentrations than TIQ and its benzyl and methyl derivatives, especially in human brain. Moreover, salsolinols were concentrated in areas with increased dopamine synthesis and turnover such as the ventral midbrain and striatum, respectively. A possible consequence of nigrostriatal dopaminergic cell death, significantly lower levels of $(R)$ salsolinol, $(S)$ salsolinol, $N$-methyl- $(R)$ salsolinol and 
$\mathrm{N}$-methyl-(S)salsolinol were found in the caudate nuclei of PD in comparison with normal human brain. This data supports the hypothesis of endogenous synthesis of salsolinols and provides evidence for their accumulation in catecholaminergic neurons. 


\section{CHAPTER 4. ANALYSIS OF TETRAHYDROISOQUINOLINE DERIVATIVES IN COMMON FRUITS AND VEGETABLES AND THEIR ACCUMULATION IN BRAIN OVER TIME}

\subsection{INTRODUCTION}

The heterocyclic alkaloids are a relatively small group of structurally related compounds composed of the isoquinolines, derived ultimately from phenylalanine, and the $\beta$-carbolines derived from tryptophan or other indolic amines (Cohen \& Collins 1970). In plants, these heterocyclic compounds arise as 1,2,3,4-tetrahydogenated derivatives (such as the TIQs) from non-enzymatic condensations of a variety of aldehydes or $\alpha$ keto acids with the above amino acids or their amine products (Collins 2002). The initial condensation step, Schiff base or imine formation, is quite ubiquitous in nature under neutral conditions and is found in reactions such as vitamin B6-dependent enzyme mechanisms and the addition of glucose molecues to various proteins. Not surprisingly, various TIQ derivatives have been detected in several common food sources derived from plants such as bananas, cocoa, flour, whiskeky, wine and beer (Deng et al 1997; Makino et al 1988; Makino et al 1990; Niwa et al 1989). TIQ derivatives have also been detected in protein-based foods such as milk, cheese, eggs, and even beef and fish, suggesting that these compounds may be taken up via dietary sources and stored in animal tissues (Makino et al 1988; Niwa et al 1989).

The detection of various TIQ derivatives in the brain, their propensity to cause cell death in neurons and their ability to cross the blood-brain barrier make them interesting candidates for an environmental or exogenous component of PD pathogenesis. Thus, diet may play an important role in the etiology of PD by altering the oxidative balance in the brain and by serving as a vehicle for environmental neurotoxins, such as the TIQs. Although genetic components may be important in determining susceptibility to early onset PD, most idiopathic PD cases are nonfamilial and most likely involve environmental risk factors (Tanner et al 1987; Tanner et al 1999). Potential roles of foods and nutrients in determining PD risk have been investigated to some extent. However, the results are largely inconsistent. Elevated risk of PD has been associated with higher intake of total energy, dietary fats, carbohydrates, monosaccharides, disaccharides, chocolates, iron and lutein; reduced risk of PD has been associated with higher intake of potatoes, nuts and foods containing niacin (Anderson et al 1999; Davies et al 1994; Fall et al 1999; Hellenbrand et al 1996a; Hellenbrand et al 1996b; Johnson et al 1999; Logroscino et al 1996; Logroscino et al 1998).

In this regard, liftetime dietary patterns may be revelant to the accumulation of dietary toxins and thus overall disease risk. Principal components analysis and dietquality scores are two commonly used dietary pattern approaches. Principal components analysis is a data-driven (a posteriori) method that describes the eating patterns of a certain population. It is based on the assumption that between-person variations can, in part, be explained by underlying unmeasured variables. Correlations between the consumption of different food items in the study population are used to identify these underlying factors or patterns, and individuals are then ranked in terms of how closely they conform to the total pattern (Hu 2002; Jacques \& Tucker 2001).

Although TIQ derivatives have been detected in a subset of foods and beverages, this data is fundamentally disorganized and incomplete. Moreover, previous 
studies have neglected much more commonly consumed plant-based food sources such as fresh fruits and vegetables. In this study, TIQ derivatives were quantified in the most commonly consumed fruits and vegetables in the United States using enantiomericselective HPLC-EC and LC-MS/MS. In addition, a dietary questionnaire was developed and administered to a population of PD patients and controls to determine if the risk of disease is associated with lifetime consumption of one or more foods items that may concentrate several TIQ derivatives. Finally, in an effort to explore the hypothesis of lifetime accumulation of TIQ derivatives in the brain, various regions of the rat brain were analyzed for TIQ content at ages ranging from 1 day to 2 years.

\subsection{MATERIALS AND METHODS}

\subsubsection{Chemicals}

Ethylenediaminetetraacetic acid (EDTA), formaldehyde, ortho-phosphoric acid, perchloric acid, sodium acetate, sodium bisulfite, sodium cyanoborohydride, triethylamine (TEA), tetramethylsilane (TMS), trifluoroacetic acid, dopamine, DOPAC, HVA, and 3,4-dihydroxybenzylamine (DHBA) were purchased from Sigma-Aldrich (St. Louis, MO, USA). Deuteriated dimethylsulfoxide (DMSO-d6) was purchased from Cambridge Isotope Labs (Woburn, MA, USA). TIQ, 1-benzyl-TIQ, (R/S)salsolinol, and norsalsolinol were purchased from Acros Organics (Geel, Belgium). 1-methyl-TIQ and $N$-methyl-TIQ were purchased from AsisChem Inc. (Cambridge, MA, USA). Sodium octylsulphonate (SOS) and monobasic anhydrous sodium dihydrogen phosphate used in mobile phase preparation were purchased from Fluka Chemie (Buchs, Switzerland). HPLC grade water, acetonitrile, and $\beta$-cyclodextrin were obtained from Fisher Scientific (Hampton, NH, USA).

\subsubsection{Synthesis of $N$-methyl-norsalsolinol and $N$-methyl-(R/S)salsolinol}

The tertiary amines $N$-methyl-norsalsolinol and $N$-methyl-(R/S)salsolinol were prepared from their respective secondary amines norsalsolinol and $(R / S)$ salsolinol by reductive methylation in the presence of formaldehyde and sodium cyanoborohydride. The reaction mixtures contained $0.5 \mathrm{M}$ of the amine, $2.5 \mathrm{M}$ formaldehyde, $0.2 \mathrm{M}$ sodium acetate, and $1 \mathrm{M}$ sodium cyanoborohydride at $\mathrm{pH}$. The reaction mixture was incubated at room temperature overnight under nitrogen in the dark. The sample was clarified by centrifugation and the clear solution was acidified with $\mathrm{HCl}$ to $\mathrm{pH} 0.1$ to decompose unreacted cyanoborohydride. The solvent was evaporated and the product was extracted from the residue with isopropyl alcohol. Evaporation of the solvent and extraction of the residue was repeated twice. The final product was stored as a solution in isopropyl alcohol at $-20{ }^{\circ} \mathrm{C}$.

\subsubsection{Procurement and Preparation of Fresh Fruits and Vegetables}

Fresh fruits $(N=20)$ and vegetables $(N=20)$ representing those most commonly consumed in the US were obtained in August 2007 (Table 4-1). Fresh products were bought on the same day at three Memphis, TN locations: an outlet of a nationwide supermarket chain, a local grocery and an open-air market. A 
Table 4-1. Plant products utilized for analysis.

\begin{tabular}{|c|c|c|c|}
\hline Common Name & Family & Common Family & Genus and Species \\
\hline Kiwi Fruit & Actinidiaceae & Actinidia Family & Actinidia chinensis \\
\hline Mushroom & Agaricaceae & Mushroom Family & Agaricus bisporus \\
\hline Green Onion & Amaryllidaceae & Amaryllis Family & Allium schoenoprasum \\
\hline Onion & Amaryllidaceae & Amaryllis Family & Allium cepa \\
\hline Carrot & Apiaceae & Carrot Family & Daucus carota \\
\hline Celery & Apiaceae & Carrot Family & Apium graveolens \\
\hline Iceberg Lettuce & Asteraceae & Sunflower Family & Lactuca sativa \\
\hline Leaf Lettuce & Asteraceae & Sunflower Family & Lactuca sativa romana \\
\hline Broccoli & Brassicaceae & Mustard Family & Brassica oleracea italica \\
\hline Cauliflower & Brassicaceae & Mustard Family & Brassica oleracea \\
\hline Green Cabbage & Brassicaceae & Mustard Family & Brassica oleracea \\
\hline Radish & Brassicaceae & Mustard Family & Raphanus sativus \\
\hline Pineapple & Bromeliaceae & Pineapple Family & Ananas comosus \\
\hline Sweet Potato & Convolvulaceae & Morning Glory Family & Ipomoea batatas \\
\hline Cantaloupe & Cucurbitaceae & Gourd Family & Cucumis melon \\
\hline Cucumber & Cucurbitaceae & Gourd Family & Cucurbita sativus \\
\hline Honeydew Melon & Cucurbitaceae & Gourd Family & Cucumis Inodorus \\
\hline Watermelon & Cucurbitaceae & Gourd Family & Citrullus lanatus \\
\hline Yellow Squash & Cucurbitaceae & Gourd Family & Cucurbita pepo \\
\hline Green Bean & Fabaceae & Pea Family & Phaseolus vulgaris \\
\hline Avocado & Lauraceae & Laurel Family & Persea americana \\
\hline Asparagus & Liliaceae & Lily Family & Asparagus officinalis \\
\hline Banana & Musaceae & Banana Family & Musa paradisiaca \\
\hline Yellow Corn & Poaceae & Grass Family & Zea mays \\
\hline Apple & Rosaceae & Rose Family & Malus sylvestris \\
\hline Cherry & Rosaceae & Rose Family & Prunus avium \\
\hline Nectarine & Rosaceae & Rose Family & Prunus persica \\
\hline Peach & Rosaceae & Rose Family & Prunus persica \\
\hline Pear & Rosaceae & Rose Family & Pyrus communis \\
\hline Plum & Rosaceae & Rose Family & Prunus domestica \\
\hline Strawberry & Rosaceae & Rose Family & Fragaria ananassa \\
\hline Grapefruit & Rutaceae & Rue Family & Citrus paradisi \\
\hline Lemon & Rutaceae & Rue Family & Citrus limon \\
\hline Lime & Rutaceae & Rue Family & Citrus aurantiifolia \\
\hline Orange & Rutaceae & Rue Family & Citrus sinensis \\
\hline Tangerine & Rutaceae & Rue Family & Citrus nobilis \\
\hline Bell Pepper & Solanaceae & Nightshade Family & Cpasicum annuum \\
\hline Potato & Solanaceae & Nightshade Family & Solanum tuberosum \\
\hline Tomato & Solanaceae & Nightshade Family & Lycopersicon esculentum \\
\hline Grape & Vitaceae & Grape Family & Vitis labrusca \\
\hline
\end{tabular}


minimum of $1 \mathrm{~kg}$ (or $0.5 \mathrm{~kg}$ for very small foods such as berries) or at least three units was sampled at each location. Fresh fruits and vegetables were collected and transported to the laboratory in clean polyethylene bags within 1 day. Each sample was identified clearly and indelibly by allocating a unique identification number. After removal of nonedible parts, samples were homogenized in $10 \mathrm{ml}$ of ice-cold dissolution buffer ( $0.1 \mathrm{M}$ perchloric acid, $0.1 \mathrm{mM}$ sodium bisulfite, and $0.1 \mathrm{mM}$ EDTA) per $1 \mathrm{~g}$ wet weight. Homogenates were then centrifuged at $20,000 \times g$ for 25 min at $4{ }^{\circ} \mathrm{C}$. Supernatants were filtered through $0.22 \mu \mathrm{m}$ pore size PVDF membrane filters. Chopping and blending of plant material was performed to ensure that the samples were homogeneous enough so that subsampling variability would be acceptable and the extracts were truly representative of the corresponding samples as a whole.

Due to the high organic content of the supernatants, solid phase extraction (SPE) was performed on all samples prior to LC-MS/MS analysis. A Waters Oasis HLB extraction cartridge ( $1 \mathrm{ml} / 30 \mathrm{mg}$; Waters Corporation, Milford, MA, USA) was conditioned with $1 \mathrm{ml}$ of methanol followed by a $1 \mathrm{ml}$ wash with water. The supernatant was loaded onto the cartridge and washed with $2 \mathrm{ml}$ deionized water and $1 \mathrm{ml} 5 \%(\mathrm{v} / \mathrm{v})$ acetonitrile with $0.1 \%(\mathrm{v} / \mathrm{v})$ trifluoroacetic acid. Analytes were eluted from the cartridge with $1 \mathrm{ml}$ of acetonitrile with $0.1 \%(\mathrm{v} / \mathrm{v})$ trifluoroacetic acid. The final solution was evaporated to dryness and the residue was reconstituted in $10 \mathrm{ml}$ of $10 \%(\mathrm{v} / \mathrm{v})$ acetonitrile.

\subsubsection{Rodent CNS Sample Preparation}

Male rats $(N=40,5$ rats per age group, standard housing and feeding) in age groups of 1 day, 14 days, 30 days, 60 days, 90 days, 365 days, 547 days and 730 days (Sprague-Dawley strain, Harlan Sprague Dawley, Inc., Indianapolis, IN, USA) were anesthetized with aerosolized isoflurane and fresh brain samples were obtained and partitioned using a dissecting microscope. Tissue samples $(0.1-0.2 \mathrm{~g})$ of striatum, ventral midbrain, frontal cerebral cortex, cerebellar cortex, and hippocampus were weighed and then homogenized in $100 \mu$ l of ice-cold dissolution buffer $(0.1 \mathrm{M}$ perchloric acid, $0.1 \mathrm{mM}$ sodium bisulfite, and $0.1 \mathrm{mM}$ EDTA) per $10 \mathrm{mg}$ wet weight. All homogenates were centrifuged at $20,000 \mathrm{~g}$ for $25 \mathrm{~min}$ at $4{ }^{\circ} \mathrm{C}$. Supernatants were filtered through $0.22 \mu \mathrm{m}$ pore size polyvinylidene fluoride (PVDF) syringe-driven membrane filters (Millipore Corp., Bedford, MA, USA) and immediately frozen and stored at $-80^{\circ} \mathrm{C}$ until the time of analysis. As above, SPE was performed on all samples prior to LC-MS/MS analysis.

\subsubsection{HPLC-EC Analysis}

\subsubsection{Chromatography Standard Preparation}

Primary stock standard solutions were prepared by dissolving $10 \mathrm{mg}$ of $(R / S) S A L, N$-methyl-(R/S)SAL, NorSAL, dopamine, DOPAC, and HVA in $25 \mathrm{ml}$ of dissolution buffer. These concentrates were then divided into $1 \mathrm{ml}$ aliquots, frozen, stored at $-80^{\circ} \mathrm{C}$, and thawed prior to use at $4^{\circ} \mathrm{C}$. Working standards in the nM range were freshly prepared prior to each assay. Standard curves employed for analyte 
quantification were generated with a DHBA internal standard. The relationship between concentration and relative response was linear over two orders of magnitude (sample correlation coefficient $>0.98$ ), with an overall electrochemical sensitivity of $0.001 \mu \mathrm{A}$. With HPLC-EC, the limit of quantification for all analytes was $0.7 \mathrm{ng} / \mathrm{ml}$.

\subsubsection{Mobile Phase Preparation}

A stock buffer solution containing $75 \mathrm{mM}$ monobasic sodium dihydrogen phosphate, $2 \mathrm{mM}$ SOS, $25 \mu \mathrm{M}$ EDTA, $20 \mathrm{mM} \beta$-cyclodextrin, and $100 \mu \mathrm{l}$ of TEA was prepared in $1800 \mathrm{ml}$ of HPLC grade water. To prepare the mobile phase, this solution was then mixed with $200 \mathrm{ml}$ of HPLC grade acetonitrile and buffered to $\mathrm{pH} 3.0$ using concentrated ortho-phosphoric acid. The mobile phase was stored at room temperature for no more than 2 days, filtered through a $0.20 \mu \mathrm{m}$ pore size white nylon filter membrane (Millipore Corp.), and degassed under vacuum for 30 min prior to use. The chiral mobile phase additive $\beta$-cyclodextrin is a cyclic oligosaccharide which preferentially binds to one face of an enantiomer. This binding changes the hydrogenbonding patterns of the enantiomers and allows for their separation using a standard reverse-phase column.

\subsubsection{Liquid Chromatography}

HPLC-EC analysis was performed with an ESA Model 5600A CoulArray ${ }^{\circledR}$ system (ESA Inc., Chemlsford, MA, USA), equipped with Shimadzu Model DGU-14A on-line degassing unit (Shimadzu Scientific Instruments, Columbia, MD, USA), an ESA Model 582 pump, and an ESA Model 542 refrigerated autosampler. The detection system consisted of three coulometric array modules, each containing four electrochemical detector cells. Electrode potentials were selected over the range of 0 to $+700 \mathrm{mV}$, with a $50 \mathrm{mV}$ increment against palladium electrodes. Chromatographic separation was achieved by auto-injecting $30 \mu \mathrm{l}$ sample aliquots at $5^{\circ} \mathrm{C}$ onto a MetaChem Intersil (MetaChem Technologies, Torrance, CA, USA) reversed-phase $\mathrm{C}_{18}$ column ( $5 \mu \mathrm{m}$ particle size, $250 X 4.6 \mathrm{~mm}$ I.D) with an ESA Hypersil $\mathrm{C}_{18}$ pre-column (5 $\mu \mathrm{m}$ particle size, $7.5 \times 4.6 \mathrm{~mm}$ I.D.) [Fig. 3A]. A mobile phase flow rate of $1.25 \mathrm{ml} / \mathrm{min}$ and analysis time of 45 min were used for all experiments. System control and data acquisition/processing were performed using ESA CoulArray software (version 1.02). All samples were processed in technical triplicate with median values used for analysis.

\subsubsection{LC-MS/MS Analysis}

\subsubsection{Chromatography Standard Preparation}

Primary stock standard solutions were prepared by dissolving $10 \mathrm{mg}$ of TIQ, 1methyl-TIQ, N-methyl-TIQ, and 1-benzyl-TIQ in $25 \mathrm{ml}$ of a mixture of $50 \%$ water and $50 \%$ acetonitrile. These concentrates were then divided into $1 \mathrm{ml}$ aliquots and stored at $-80^{\circ} \mathrm{C}$. Working standards in the $\mathrm{nM}$ range were freshly prepared prior to each assay. 


\subsubsection{Liquid Chromatography}

LC-MS/MS analysis was performed using an Agilent model 1100 series HPLC system equipped with a Bruker Esquire-LC ion trap tandem mass selective detector (Bruker Daltronics Inc., Billerica, MA, USA), a Model 1327A temperature controlled autosampler, a Model 1316A temperature controlled column compartment, and a Model G1312A binary pump with Model G1322A solvent degassing module, and a Model G1314A variable wavelength UV-vis detector (Agilent Technologies, Santa Clara, CA). Ultraviolet absorbance monitoring was performed at $220 \mathrm{~nm}$. Separation was achieved by auto-injecting $10 \mu \mathrm{l} \mathrm{sample} \mathrm{aliquots} \mathrm{at} 5^{\circ} \mathrm{C}$ onto a Thermo Hypersil-Keystone reversed-phase $\mathrm{C}_{18}$ column ( $5 \mu \mathrm{m} ; 50 \times 2.1 \mathrm{~mm}$ I.D.; Thermo Fisher Scientific, Waltham, MA, USA) in combination with a $\mathrm{C}_{18}$ Hypersil guard column $(10 \times 2.1 \mathrm{~mm}$ I.D.). A binary mobile phase flow rate of $0.7 \mathrm{ml} / \mathrm{min}$ and analysis time of $25 \mathrm{~min}$ was utilized. Mobile phase A consisted of water with $0.1 \%$ formic acid and mobile phase B consisted of acetonitrile with $0.1 \%$ formic acid. The following mobile phase gradient was used: $0 \mathrm{~min}$ $A / B=95: 5,5 \mathrm{~min} A / B=70: 30,10 \min A / B=50: 50,15 \mathrm{~min} A / B=70: 30,20 \mathrm{~min} A / B=$ 95:5. The column was given a 5 min post-run period to allow for re-equilibration. System control and data acquisition/processing were performed using ChemStation software revision A.08.03 for LC-MS systems (Agilent Technologies).

\subsubsection{Ion Trap Tandem Mass Spectrometry}

Full-scan mode was used to acquire mass spectra, precursor ions, and product ions. EsquireControl software version 6.16 (Bruker Daltronics Inc.) was employed for control of all mass spectrometric conditions. The mass spectrometer was equipped with a heated capillary interface and an electrospray ionization (ESI) source, operating in positive ion mode. The ESI source and MS/MS parameters were automatically optimized. Spray needle voltage was $4.0 \mathrm{kV}$ and capillary temperature was set to $220^{\circ} \mathrm{C}$. Sheath and auxiliary nitrogen gas flow were set to 40 and $0 \mathrm{psi}$, respectively. Automatic gain control was utilized with a maximum isolation time of $300 \mathrm{~ms}$. A relative collision energy of $33 \%$ was used for all analyses. The product ions producing the highest intensity were used for quantification to increase analytical sensitivity and

selectivity in LC-MS/MS mode. Quantification was based on detector response, defined as the ratio of the base peak ion of interest to the base peak ion of the standard compound. The relationship between concentration and relative response was linear over two orders of magnitude (sample correlation coefficient > 0.998) for the MS/MS procedure. All samples were processed in technical triplicate with median values used for statistical analyses. With LC-MS/MS, $0.3 \mathrm{ng} / \mathrm{ml}$ was the limit of quantification for all analytes.

\subsubsection{Parkinson Disease Lifetime Food Intake Questionnaire}

\subsubsection{Instrument Development}

As an extension of the results from chromatographic analysis of TIQ derivatives in fresh fruits and vegetables, a lifetime food-serving-intake questionnaire was 
developed to assess the eating habits of PD patients based on servings of specific individual foods (i.e. the top 20 most commonly consumed fruits and vegetables in the US) and to search for correlations between the intake of certain fruits and vegetables and the development of idiopathic PD. This survey (see Appendix B), which requires approximately 30-45 min to complete, quantifies the frequency and servings of specific raw or cooked fruits and vegetables consumed over specific decades of a participant's life, as well as overall lifetime intake. Data was obtained from PD patients and age- and gender-matched controls over a period of three years. Patients with a diagnosis of PD were recruited from clinics and hospitals in the greater Memphis, TN area.

\subsubsection{Instrument Statistical Analysis}

Multivariate analysis was performed via orthogonal linear transformation and determination of variance along principle axes (principal component analysis). The principal axis method was used to extract all components, followed by a varimax rotation. By convention, questionnaire items with eigenvalues greater than 1.000 were considered principal components of the survey. Confidence intervals (95\%) were calculated for each level of intake (0-20 yrs, 21-40 yrs, 41-60 yrs, and >60 yrs) and for average monthly lifetime intake for each food item.

\subsubsection{Reproducibility and Validity}

Reproducibility and validity were evaluated using methods common to dietary assessment questionnaires (Block \& Hartman 1989; Buzzard et al 2001; Dennison et al 2000; Hoelscher et al 2003). Reproducibility (or reliability) is the ability of an instrument to produce the same estimate on two separate occasions, assuming no interim changes. Validity can be defined as the ability of an instrument to accurately measure its intended subject. Cross-sectional reproducibility was determined using a re-administration of the questionnaire to $20 \mathrm{PD}$ and 20 control subjects at a 6 month interval. Cross-sectional validity was determined using a simple one month food diary completed by 20 PD and 20 control subjects. Reproducibility and validity was assessed by calculating Spearman rank order correlation coefficients and Cohen kappa (к) statistics. Wilcoxon matchedpair signed rank tests were used to determine if a significant difference existed between food intakes reported at the first and second questionnaire administration (for reproducibility) and between reported questionnaire intake values and food diary recall values for specific foods under each subject's appropriate age category (for validation).

\subsection{RESULTS}

\subsubsection{TIQ Derivatives in Common Fruits and Vegetables}

As shown in Tables 4-2 and 4-3, TIQ derivatives were detected in all fruits and vegetables subjected to analysis. As shown in Table 4-4 and 4-5, derivatives of TIQ (TIQ, 1-methyl-TIQ, N-methyl-TIQ and 1-benzyl-TIQ) were detected in particularly high concentrations in banana, cherry, grapefruit, avocado, button mushroom, leaf lettuce and celery. Derivatives of salsolinol ((R/S)SAL, NorSAL, $N$-methyl-(R/S)SAL and 
Table 4-2. Concentrations of TIQ derivatives in common fruits.

\begin{tabular}{lccccc}
\hline Fruit & TIQ & 1-methyl-TIQ & N-methyl-TIQ & 1-benzyl-TIQ & NorSAL \\
\hline Banana & $984.35+/-10.60$ & $255.83+/-17.46$ & $307.64+/-28.94$ & $125.64+/-15.33$ & $1422.52+/-5.11$ \\
Apple & $167.31+/-9.11$ & $34.58+/-6.32$ & $27.06+/-3.20$ & ND & $141.81+/-6.49$ \\
Watermelon & $70.25+/-3.55$ & ND & ND & ND & $117.31+/-7.78$ \\
Orange & $54.06+/-3.10$ & $12.62+/-1.40$ & $17.38+/-2.61$ & ND & $21.92+/-1.39$ \\
Cantaloupe & $26.94+/-2.55$ & ND & $3.19+/-0.89$ & $1.54+/-0.30$ & $112.4+/-5.26$ \\
Grape & $257.06+/-9.16$ & ND & ND & ND & $532.23+/-12.49$ \\
Grapefruit & $462.11+/-12.30$ & $18.71+/-3.21$ & $13.88+/-1.96$ & $4.64+/-0.85$ & $16.91+/-1.5$ \\
Strawberry & $107.05+/-10.20$ & ND & $2.16+/-0.67$ & ND & $217.81+/-8.94$ \\
Peach & $185.64+/-6.33$ & $26.08+/-3.64$ & $17.03+/-1.48$ & ND & $75.11+/-1.65$ \\
Pear & $16.11+/-5.22$ & ND & ND & ND & $69.39+/-4.42$ \\
Nectarine & $74.02+/-5.79$ & $9.11+/-1.63$ & $3.24+/-1.05$ & ND & $100.52+/-7.02$ \\
Honeydew Melon & $60.20+/-4.21$ & ND & $2.91+/-0.68$ & ND & $71.71+/-8.12$ \\
Plum & $119.06+/-7.02$ & $10.60+/-2.18$ & $19.57+/-1.56$ & $13.66+/-3.43$ & $75.11+/-1.65$ \\
Avocado & $455.06+/-12.10$ & $40.91+/-3.22$ & $38.47+/-4.02$ & $22.31+/-4.17$ & $401.73+/-10.65$ \\
Lemon & $331.12+/-7.20$ & $15.29+/-0.78$ & $6.35+/-1.02$ & $8.44+/-1.30$ & $25.13+/-9.32$ \\
Pineapple & $120.98+/-50.03$ & ND & $14.36+/-2.44$ & ND & $95.96+/-5.64$ \\
Tangerine & $261.35+/-12.00$ & $67.73+/-5.82$ & $96.35+/-9.02$ & ND & $16.78+/-5.69$ \\
Cherry & $616.24+/-22.09$ & $5.33+/-0.88$ & $7.13+/-1.77$ & $2.06+/-0.80$ & $41.75+/-9.69$ \\
Kiwi Fruit & $184.33+/-8.10$ & $33.14+/-4.35$ & $21.09+/-3.05$ & ND & $266.45+/-90$ \\
Lime & $368.26+/-4.68$ & $38.06+/-3.69$ & $17.93+/-1.17$ & ND & $55.45+/-3.56$
\end{tabular}


Table 4-2. (Continued).

\begin{tabular}{lccccc}
\hline Fruit & $(R) S A L$ & $(S) S A L$ & $N$-methyl-(R)SAL & $N$-methyl-(S)SAL & $\boldsymbol{N}$-methyl-NorSAL \\
\hline Banana & $2717.50+/-9.81$ & $2870.87+/-10.95$ & $1102.16+/-12.25$ & $1133.59+/-7.86$ & $1977.53+/-3.22$ \\
Apple & $29.63+/-0.25$ & $30.41+/-1.72$ & $16.05+/-1.55$ & $17.10+/-0.17$ & $309.93+/-6.21$ \\
Watermelon & $21.92+/-0.86$ & $25.66+/-0.77$ & $9.34+/-0.38$ & $10.81+/-0.26$ & $81.59+/-5.65$ \\
Orange & $232.34+/-4.79$ & $246.94+/-3.77$ & $280.90+/-5.11$ & $287.86+/-3.28$ & $13.89+/-0.93$ \\
Cantaloupe & $18.53+/-0.91$ & $17.94+/-0.67$ & $35.78+/-2.26$ & $34.835+/-1.84$ & $48.54+/-5.89$ \\
Grape & $951.62+/-11.71$ & $980.84+/-12.96$ & $\mathrm{ND}$ & $\mathrm{ND}$ & $154.25+/-9.15$ \\
Grapefruit & $419.19+/-4.66$ & $404.86+/-3.87$ & $611.02+/-17.5$ & $632.53+/-24.82$ & $715.48+/-15.04$ \\
Strawberry & $389.8+/-7.35$ & $362.28+/-9.07$ & $74.68+/-8.12$ & $81.28+/-5.07$ & $112.48+/-8.83$ \\
Peach & $31.85+/-9.51$ & $39.46+/-5.54$ & $7008.16+/-26.4$ & $7130.39+/-11.22$ & $5.79+/-0.88$ \\
Pear & $34.09+/-1.44$ & $35.18+/-9.74$ & $4516.3+/-77.10$ & $4670.65+/-63.32$ & $5.76+/-0.31$ \\
Nectarine & $48.76+/-4.26$ & $46.43+/-2.97$ & $589.33+/-12.89$ & $595.20+/-16.21$ & $14.66+/-2.30$ \\
Honeydew Melon & $9.63+/-4.31$ & $8.82+/-1.27$ & $206.27+/-8.16$ & $197.95+/-4.41$ & $101.72+/-6.92$ \\
Plum & $\mathrm{ND}$ & $\mathrm{ND}$ & $280.99+/-5.11$ & $287.86+/-3.28$ & $154.25+/-9.15$ \\
Avocado & $334.44+/-8.62$ & $328.26+/-7.57$ & $305.31+/-1.61$ & $292.42+/-7.71$ & $\mathrm{ND}$ \\
Lemon & $282.33+/-12.66$ & $289.20+/-8.51$ & $81.33+/-4.02$ & $75.92+/-8.03$ & $\mathrm{ND}$ \\
Pineapple & $48.16+/-13.06$ & $47.17+/-18.03$ & $1250.07+/-16.22$ & $1318.66+/-11.85$ & $322.83+/-20.3$ \\
Tangerine & $238.43+/-5.41$ & $237.25+/-8.49$ & $791.66+/-21.08$ & $804.36+/-18.56$ & $481.32+/-12.17$ \\
Cherry & $16.73+/-3.44$ & $12.03+/-4.77$ & $6103.49+/-18.78$ & $6180.03+/-27.48$ & $16.92+/-1.41$ \\
Kiwi Fruit & $114.65+/-7.28$ & $120.32+/-2.46$ & $540.62+/-11.60$ & $551.27+/-5.35$ & $174.49+/-5.90$ \\
Lime & $107.24+/-5.52$ & $118.165+/-9.21$ & $1167.33+/-15.64$ & $1251.45+/-27.93$ & $126.46+/-4.34$ \\
\hline
\end{tabular}

Values are expressed in ng/g wet weight.

ND, Not Detected. 
Table 4-3. Concentrations of TIQ derivatives in common vegetables.

\begin{tabular}{lccccc}
\hline \multicolumn{1}{c}{ Vegetable } & TIQ & 1-methyl-TIQ & N-methyl-TIQ & 1-benzyl-TIQ & NorSAL \\
\hline Potato & $52.16+/-4.55$ & $\mathrm{ND}$ & $2.57+/-0.45$ & $\mathrm{ND}$ & $289.72+/-5.01$ \\
lceberg Lettuce & $89.22+/-7.40$ & $\mathrm{ND}$ & $8.24+/-1.32$ & $\mathrm{ND}$ & $114.18+/-9.34$ \\
Tomato & $134.11+/-4.61$ & $9.63+/-2.11$ & $\mathrm{ND}$ & $\mathrm{ND}$ & $28.47+/-3.70$ \\
Onion & $16.44+/-2.33$ & $\mathrm{ND}$ & $3.28+/-1.60$ & $1.57+/-0.40$ & $50.43+/-6.46$ \\
Carrot & $24.61+/-4.41$ & $\mathrm{ND}$ & $\mathrm{ND}$ & $\mathrm{ND}$ & $29.05+/-2.55$ \\
Celery & $467.66+/-20.10$ & $8.46+/-1.66$ & $12.44+/-2.28$ & $5.13+/-0.23$ & $9.66+/-0.72$ \\
Yellow Corn & $31.61+/-3.10$ & $\mathrm{ND}$ & $\mathrm{ND}$ & $\mathrm{ND}$ & $94.78+/-3.36$ \\
Broccoli & $49.13+/-4.66$ & $\mathrm{ND}$ & $1.86+/-0.33$ & $\mathrm{ND}$ & $100.22+/-3.10$ \\
Green Cabbage & $55.49+/-8.20$ & $3.83+/-0.87$ & $5.16+/-0.75$ & $1.60+/-0.40$ & $60.67+/-5.55$ \\
Cucumber & $18.26+/-2.62$ & $\mathrm{ND}$ & $\mathrm{ND}$ & $\mathrm{ND}$ & $27.56+/-5.25$ \\
Bell Pepper & $167.03+/-10.3$ & $6.73+/-1.03$ & $4.50+/-1.03$ & $3.67+/-0.56$ & $187.67+/-4.1$ \\
Cauliflower & $19.46+/-4.09$ & $\mathrm{ND}$ & $2.67+/-0.98$ & $\mathrm{ND}$ & $26.21+/-8.77$ \\
Leaf Lettuce & $493.54+/-16.20$ & $15.33+/-2.45$ & $12.11+/-3.00$ & $7.99+/-1.37$ & $167.91+/-5.08$ \\
Sweet Potato & $136.07+/-6.46$ & $4.92+/-0.96$ & $9.34+/-1.25$ & $6.27+/-0.87$ & $97.14+/-5.84$ \\
Mushroom & $1314.56+/-13.15$ & $194.24+/-12.44$ & $276.04+/-32.62$ & $67.16+/-8.20$ & $2174.58+/-27.92$ \\
Green Onion & $87.01+/-6.14$ & $\mathrm{ND}$ & $2.47+/-0.72$ & $\mathrm{ND}$ & $147.55+/-9.71$ \\
Green Bean & $152.68+/-7.13$ & $\mathrm{ND}$ & $\mathrm{ND}$ & $\mathrm{ND}$ & $51.92+/-9.60$ \\
Radish & $14.08+/-2.10$ & $2.39+/-0.83$ & $4.39+/-1.23$ & $\mathrm{ND}$ & $39.86+/-1.37$ \\
Yellow Squash & $416.94+/-17.35$ & $8.55+/-1.62$ & $14.45+/-3.41$ & $12.48+/-2.50$ & $31.63+/-7.61$ \\
Asparagus & $232.02+/-14.50$ & $7.32+/-1.46$ & $11.04+/-5.21$ & $8.10+/-1.06$ & $565.37+/-6.57$
\end{tabular}


Table 4-3. (Continued).

\begin{tabular}{lccccc}
\hline Vegetable & $(R) S A L$ & $(S) S A L$ & $N$-methyl-(R)SAL & $\boldsymbol{N}$-methyl-(S)SAL & $\boldsymbol{N}$-methyl-NorSAL \\
\hline Potato & $77.81+/-8.19$ & $88.79+/-5.68$ & ND & ND & $67.11+/-5.71$ \\
Iceberg Lettuce & $16.85+/-6.10$ & $18.35+/-3.89$ & $22.56+/-3.02$ & $19.06+/-6.87$ & $101.22+/-3.01$ \\
Tomato & $54.84+/-6.38$ & $56.55+/-3.69$ & $311.58+/-16.11$ & $300.83+/-11.50$ & $80.16+/-7.73$ \\
Onion & $6.05+/-1.62$ & $4.9+/-1.57$ & $26.08+/-2.66$ & $23.18+/-8.01$ & $0.89+/-0.03$ \\
Carrot & $42.91+/-1.30$ & $40.61+/-5.83$ & $15.22+/-2.69$ & $14.96+/-1.61$ & $24.13+/-2.53$ \\
Celery & $1372.85+/-15.60$ & $1382.01+/-12.03$ & $232.83+/-9.20$ & $239.64+/-7.48$ & $1355.56+/-29.33$ \\
Yellow Corn & $86.47+/-4.35$ & $80.44+/-6.90$ & $37.69+/-2.21$ & $31.46+/-5.64$ & $25.30+/-1.25$ \\
Broccoli & $44.84+/-2.31$ & $49.56+/-6.93$ & $2.44+/-0.20$ & $6.94+/-0.63$ & $35.24+/-2.04$ \\
Green Cabbage & $35.46+/-3.87$ & $38.48+/-5.63$ & $21.08+/-3.13$ & $27.58+/-6.52$ & $32.76+/-6.77$ \\
Cucumber & $12.87+/-1.52$ & $13.66+/-0.98$ & $11.10+/-2.61$ & $9.81+/-1.74$ & $17.09+/-2.25$ \\
Bell Pepper & $59.52+/-4.90$ & $61.77+/-6.32$ & $33.64+/-4.03$ & $29.68+/-6.37$ & $12.10+/-2.48$ \\
Cauliflower & $13.16+/-1.35$ & $14.23+/-2.26$ & $30.55+/-6.20$ & $36.89+/-4.07$ & $22.89+/-2.09$ \\
Leaf Lettuce & $2615.23+/-42.35$ & $2660.49+/-33.55$ & $627.63+/-8.33$ & $631.56+/-11.95$ & $2149.28+/-27.25$ \\
Sweet Potato & $295.23+/-5.87$ & $286.80+/-8.49$ & $317.09+/-12.50$ & $310.97+/-8.68$ & $68.20+/-6.58$ \\
Mushroom & $3572.80+/-13.44$ & $3557.40+/-17.48$ & $5998.02+/-33.10$ & $6026.26+/-25.6$ & $595.29+/-18.39$ \\
Green Onion & $255.72+/-9.92$ & $263.59+/-8.82$ & $97.50+/-12.43$ & $106.69+/-9.28$ & $38.88+/-9.33$ \\
Green Bean & $195.17+/-9.31$ & $215.58+/-6.89$ & $322.34+/-4.22$ & $330.41+/-6.57$ & $277.47+/-3.93$ \\
Radish & $19.81+/-1.26$ & $21.06+/-1.41$ & $\mathrm{ND}$ & $\mathrm{ND}$ & $22.11+/-0.36$ \\
Yellow Squash & $152.41+/-6.52$ & $151.16+/-8.03$ & $102.54+/-8.26$ & $110.98+/-6.78$ & $555.63+/-12.75$ \\
Asparagus & $104.82+/-18.44$ & $101.43+/-12.83$ & $177.03+/-16.21$ & $169.63+/-13.43$ & $47.95+/-3.05$ \\
\hline
\end{tabular}

Values are expressed in ng/g wet weight.

ND, Not Detected. 
Table 4-4. Total neurotoxin load by fruit.

\begin{tabular}{lccc}
\hline Fruit & TIQ Load & Salsolinol Load & Total Neurotoxin Load \\
\hline Peach & $228.75+/-11.45$ & $14290.76+/-55.20$ & $14519.51+/-66.65$ \\
Cherry & $630.76+/-25.54$ & $12370.95+/-65.57$ & $13001.71+/-91.11$ \\
Banana & $1673.46+/-72.33^{*}$ & $11224.17+/-49.20$ & $12897.63+/-121.53$ \\
Pear & $16.11+/-5.22$ & $9331.37+/-156.33$ & $9347.48+/-161.55$ \\
Grapefruit & $499.34+/-18.32$ & $2799.99+/-67.39$ & $3299.33+/-85.71$ \\
Lime & $424.25+/-9.54$ & $2826.09+/-66.20$ & $3250.34+/-75.74$ \\
Pineapple & $135.34+/-52.47$ & $3082.85+/-85.10$ & $3218.19+/-137.57$ \\
Tangerine & $425.43+/-26.84$ & $2569.80+/-71.40$ & $2995.23+/-98.24$ \\
Grape & $257.06+/-9.16$ & $2618.94+/-46.31$ & $2876.00+/-55.47$ \\
Avocado & $556.75+/-23.51$ & $1662.16+/-36.16$ & $2218.91+/-59.67$ \\
Kiwi Fruit & $238.56+/-15.50$ & $1767.80+/-122.59$ & $2006.36+/-138.09$ \\
Nectarine & $86.37+/-8.47$ & $1394.90+/-45.65$ & $1481.27+/-54.12$ \\
Strawberry & $109.21+/-10.87$ & $1238.33+/-47.38$ & $1347.54+/-58.25$ \\
Orange & $84.06+/-7.11$ & $1083.85+/-19.27$ & $1167.91+/-26.38$ \\
Lemon & $361.20+/-10.30$ & $753.91+/-42.54$ & $1115.11+/-52.84$ \\
Plum & $162.89+/-14.19$ & $798.21+/-19.19$ & $961.10+/-33.38$ \\
Apple & $228.95+/-18.63$ & $544.93+/-16.39$ & $773.88+/-35.02$ \\
Honeydew Melon & $63.11+/-4.89$ & $596.10+/-33.19$ & $659.21+/-38.08$ \\
Watermelon & $70.25+/-3.55$ & $266.63+/-15.70$ & $336.88+/-19.25$ \\
Cantaloupe & $31.67+/-3.74$ & $268.02+/-16.83$ & $299.695+/-20.57$ \\
\hline
\end{tabular}

Values are expressed in ng/g wet weight. 
Table 4-5. Total neurotoxin load by vegetable.

\begin{tabular}{lccc}
\hline Vegetable & TIQ Load & Salsolinol Load & Total Neurotoxin Load \\
\hline Mushroom & $1852.00+/-66.41$ & $21924.35+/-135.93$ & $23776.35+/-202.34$ \\
Leaf Lettuce & $528.97+/-23.02$ & $8852.10+/-128.51$ & $9381.07+/-151.53$ \\
Celery & $493.69+/-24.27$ & $4592.55+/-74.36$ & $5086.24+/-98.63$ \\
Yellow Squash & $452.42+/-24.88$ & $1104.35+/-49.95$ & $1556.77+/-74.83$ \\
Green Bean & $152.68+/-7.13$ & $1392.89+/-40.52$ & $1545.57+/-47.65$ \\
Sweet Potato & $156.60+/-9.54$ & $1375.43+/-47.96$ & $1532.03+/-57.50$ \\
Asparagus & $258.48+/-22.23$ & $1166.23+/-70.53$ & $1424.71+/-92.76$ \\
Green Onion & $89.48+/-6.86$ & $909.93+/-59.49$ & $999.41+/-66.35$ \\
Tomato & $143.74+/-6.72$ & $832.43+/-49.11$ & $976.17+/-55.83$ \\
Potato & $54.73+/-5.00$ & $523.43+/-24.59$ & $578.16+/-29.59$ \\
Bell Pepper & $181.93+/-12.92$ & $384.38+/-28.20$ & $566.31+/-41.12$ \\
Iceberg Lettuce & $97.46+/-8.72$ & $292.22+/-32.23$ & $389.68+/-40.95$ \\
Yellow Corn & $31.61+/-3.10$ & $356.14+/-23.71$ & $387.75+/-26.81$ \\
Broccoli & $50.99+/-4.99$ & $239.24+/-15.21$ & $290.23+/-20.20$ \\
Green Cabbage & $66.08+/-10.22$ & $216.03+/-31.47$ & $282.11+/-41.69$ \\
Carrot & $24.61+/-4.41$ & $166.88+/-16.51$ & $191.49+/-20.92$ \\
Cauliflower & $22.13+/-5.07$ & $143.93+/-24.74$ & $166.06+/-29.81$ \\
Onion & $21.29+/-4.33$ & $111.53+/-20.35$ & $132.82+/-24.68$ \\
Radish & $20.86+/-4.16$ & $102.84+/-4.40$ & $123.70+/-8.56$ \\
Cucumber & $18.26+/-2.62$ & $92.09+/-14.35$ & $110.35+/-16.97$ \\
\hline
\end{tabular}

Values are expressed in ng/g wet weight. 
$\mathrm{N}$-methyl-NorSAL) were detected in particularly high concentrations in peach, cherry, banana, pear, mushroom, leaf lettuce, celery and green bean. In general, hydroxylated and methylated derivatives were present at higher concentrations than their parent TIQ.

In contrast, TIQ was present at much higher concentrations than 1-benzyl-TIQ. $N$-methylated derivatives of salsolinol ( $N$-methyl-(R/S)SAL and $N$-methyl-NorSAL) were typically found in much higher concentrations than their parent $(R / S) S A L$. However, this effect was more pronounced in fruit species. Interestingly, enantiomeric salsolinol derivatives were detected as racemic mixtures in all plant species, with no evidence of stereoselective synthesis. In general, total toxin load was higher in fruits than in vegetables. However, the plant with the highest total toxin load was button mushroom.

As shown in Table 4-6, the mushroom family (Agaricaceae) contained the highest average TIQ derivative load, salsolinol derivative load and total toxin load of all plant families subjected to analysis. The banana family (Musaceae), rose family (Rosaceae), sunflower family (Asteraceae) and pineapple family (Bromeliaceae) also contained particularly high total average neurotoxin loads. In general, plant families contained significantly higher levels of salsolinol derivatives than TIQ derivatives, most of which were an order of magnitude greater (rose, sunflower, pineapple, grape, carrot, rue, pea, morning glory, lily, amaryllis and grass families). For most plants, total neurotoxin load was detected on the part per million scale ( $\mu \mathrm{g} / \mathrm{g}$ tissue).

The lowest average TIQ derivative, salsolinol derivative and total toxin loads were detected in plants from the mustard (Brassicaceae), grass (Poaceae), amaryllis (Amaryllidaceae) and gourd (Cucurbitaceae) families. Plant families with intermediate average toxin levels included the nightshade (Solanaceae), lilly (Liliaceae), pea (Fabaceae), laurel (Lauraceae), rue (Rutaceae), carrot (Apiaceae) and grape (Vitaceae).

\subsubsection{Lifetime Intake of Fresh Fruits and Vegetables in PD Patients}

We received survey responses from 183 PD cases in men and 102 in women (mean age $66+/-10$ years). Of the normal human respondents, 137 were male and 148 were female (mean age $68+/-11$ years). Major dietary factors were identified (Table 4-7 and 4-8) in both PD and normal human respondents from principal components analysis of questionnaire data: the intake of 7 fruit items and 6 vegetable items. One dietary pattern was characterized by high intakes of banana, apple, watermelon, orange, cantaloupe, grape and grapefruit (relative to other fruits); the other dietary pattern was characterized by high intakes potato, iceberg lettuce, tomato, onion, carrot and celery (relative to other vegetables).

Examination of Table 4-9 reveals a trend in higher intake of apple, watermelon, orange, cantaloupe, grape and grapefruit in PD patients relative to controls. However, only lifetime monthly intake or oranges reached statistical significance $(P<0.02)$. A trend of higher intake of potato, iceberg lettuce, tomato and carrot in PD patients was also observed. However, these differences did not reach statistical significance. 
Table 4-6. Average neurotoxin load by plant family.

\begin{tabular}{lcccc}
\hline Family & Common Name & TIQ Load & Salsolinol Load & Total Neurotoxin Load \\
\hline Agaricaceae & Mushroom Family & $1852.00+/-66.41$ & $21924.35+/-135.93$ & $23776.35+/-202.34$ \\
Musaceae & Banana Family & $1673.46+/-72.33$ & $11224.17+/-49.20$ & $12897.63+/-121.53$ \\
Rosaceae & Rose Family & $209.01+/-13.48$ & $5709.92+/-57.96$ & $5918.93+/-71.44$ \\
Asteraceae & Sunflower Family & $313.22+/-15.87$ & $4572.16+/-80.37$ & $4885.38+/-96.24$ \\
Bromeliaceae & Pineapple Family & $135.34+/-52.47$ & $3082.85+/-85.10$ & $3218.19+/-137.57$ \\
Vitaceae & Grape Family & $257.06+/-9.16$ & $2618.94+/-46.31$ & $2876.00+/-55.47$ \\
Apiaceae & Carrot Family & $259.15+/-14.34$ & $2379.72+/-45.44$ & $2638.87+/-59.78$ \\
Rutaceae & Rue Family & $358.86+/-14.42$ & $2006.73+/-53.36$ & $2365.59+/-67.78$ \\
Lauraceae & Laurel Family & $556.75+/-23.51$ & $1662.16+/-36.16$ & $2218.91+/-59.67$ \\
Actinidiaceae & Actinidia Family & $238.56+/-15.50$ & $1767.80+/-122.59$ & $2006.36+/-138.09$ \\
Fabaceae & Pea Family & $152.68+/-7.13$ & $1392.89+/-40.52$ & $1545.57+/-47.65$ \\
Convolvulaceae & Morning Glory Family & $156.60+/-9.54$ & $1375.43+/-47.96$ & $1532.03+/-57.50$ \\
Liliaceae & Lily Family & $258.48+/-22.23$ & $1166.23+/-70.53$ & $1424.71+/-92.76$ \\
Solanaceae & Nightshade Family & $126.80+/-8.21$ & $580.08+/-33.97$ & $706.88+/-42.18$ \\
Cucurbitaceae & Gourd Family & $127.14+/-7.94$ & $465.44+/-26.00$ & $592.58+/-33.94$ \\
Amaryllidaceae & Amaryllis Family & $55.39+/-5.60$ & $510.73+/-39.92$ & $566.12+/-45.52$ \\
Poaceae & Grass Family & $31.61+/-3.10$ & $356.14+/-23.71$ & $387.75+/-26.81$ \\
Brassicaceae & Mustard Family & $40.02+/-6.11$ & $175.51+/-18.96$ & $215.53+/-25.07$ \\
\hline
\end{tabular}

Values are expressed in ng/g wet weight. 
Table 4-7. Principal components analysis of fruit items.

\begin{tabular}{lcccc}
\hline Fruit & $\begin{array}{c}\text { Lifetime Mean } \\
\text { (Servings/Month) }\end{array}$ & Standard Error & Eigenvalue* $\%$ of Variance \\
\hline Banana & 3.900 & 0.119 & 5.204 & 26.016 \\
Apple & 5.286 & 0.145 & 2.071 & 10.356 \\
Watermelon & 2.845 & 0.083 & 1.877 & 9.384 \\
Orange & 5.020 & 0.132 & 1.563 & 7.816 \\
Cantaloupe & 2.538 & 0.074 & 1.167 & 5.837 \\
Grape & 2.993 & 0.090 & 1.071 & 5.355 \\
Grapefruit & 2.369 & 0.064 & 1.010 & 5.052 \\
Strawberry & 2.653 & 0.083 & 0.937 & 4.683 \\
Peach & 2.574 & 0.072 & 0.761 & 3.806 \\
Pear & 1.735 & 0.063 & 0.610 & 3.051 \\
Nectarine & 0.619 & 0.020 & 0.594 & 2.972 \\
Honeydew Melon & 1.254 & 0.030 & 0.518 & 2.592 \\
Plum & 1.103 & 0.028 & 0.446 & 2.232 \\
Avocado & 1.001 & 0.047 & 0.393 & 1.965 \\
Lemon & 2.120 & 0.104 & 0.374 & 1.869 \\
Pineapple & 1.496 & 0.051 & 0.328 & 1.642 \\
Tangerine & 0.796 & 0.024 & 0.317 & 1.585 \\
Cherry & 0.997 & 0.032 & 0.269 & 1.343 \\
Kiwi Fruit & 0.417 & 0.031 & 0.252 & 1.260 \\
Lime & 0.701 & 0.023 & 0.236 & 1.181 \\
\hline
\end{tabular}

* Eigenvalues $>1.000$ considered principal components. 
Table 4-8. Principal components analysis of vegetable items.

\begin{tabular}{lcccc}
\hline Vegetable & $\begin{array}{c}\text { Lifetime Mean } \\
\text { (Servings/Month) }\end{array}$ & Standard Error & Eigenvalue* $\%$ of Variance \\
\hline Potato & 10.386 & 0.176 & 4.915 & 24.573 \\
Iceberg Lettuce & 9.437 & 0.197 & 1.909 & 9.546 \\
Tomato & 6.469 & 0.156 & 1.708 & 8.538 \\
Onion & 5.263 & 0.133 & 1.342 & 6.708 \\
Carrot & 4.560 & 0.117 & 1.269 & 6.347 \\
Celery & 4.518 & 0.106 & 1.055 & 5.275 \\
Yellow Corn & 5.985 & 0.133 & 0.916 & 4.582 \\
Broccoli & 2.994 & 0.085 & 0.880 & 4.402 \\
Green Cabbage & 3.864 & 0.097 & 0.805 & 4.024 \\
Cucumber & 3.731 & 0.088 & 0.722 & 3.611 \\
Bell Pepper & 2.595 & 0.069 & 0.586 & 2.930 \\
Cauliflower & 1.037 & 0.035 & 0.543 & 2.715 \\
Leaf Lettuce & 3.818 & 0.123 & 0.528 & 2.638 \\
Sweet Potato & 2.979 & 0.084 & 0.521 & 2.603 \\
Mushroom & 1.310 & 0.080 & 0.479 & 2.395 \\
Green Onion & 2.709 & 0.095 & 0.437 & 2.184 \\
Green Bean & 6.168 & 0.135 & 0.416 & 2.081 \\
Radish & 1.363 & 0.077 & 0.376 & 1.881 \\
Yellow Squash & 2.094 & 0.063 & 0.342 & 1.709 \\
Asparagus & 2.280 & 0.065 & 0.252 & 1.260 \\
\hline
\end{tabular}

* Eigenvalues $>1.000$ considered principal components. 
Table 4-9. 95\% confidence intervals for principal components (mean lifetime monthly intake).

\begin{tabular}{lccc}
\hline Food Item & NH 95\% Cl & PD 95\% Cl & $P$ Value \\
\hline Banana & $3.71-4.50$ & $2.57-4.10$ & 0.12 \\
Apple & $4.97-5.49$ & $4.80-5.89$ & 0.38 \\
Watermelon & $2.59-3.00$ & $2.64-3.13$ & 0.54 \\
Orange & $4.42-4.75$ & $4.92-5.80$ & $0.02^{*}$ \\
Cantaloupe & $2.25-2.57$ & $2.43-2.91$ & 0.09 \\
Grape & $2.76-3.22$ & $2.72-3.27$ & 0.80 \\
Grapefruit & $2.15-2.52$ & $2.33-2.67$ & 0.62 \\
& & & \\
Potato & $9.81-10.67$ & $9.98-11.07$ & 0.41 \\
Iceberg Lettuce & $8.74-9.80$ & $9.04-10.17$ & 0.40 \\
Tomato & $5.94-6.76$ & $6.12-7.05$ & 0.67 \\
Onion & $4.96-5.78$ & $4.82-5.48$ & 0.43 \\
Carrot & $4.12-4.73$ & $4.35-5.04$ & 0.29 \\
Celery & $4.25-4.82$ & $4.19-4.81$ & 0.81 \\
\hline
\end{tabular}

$\mathrm{NH}$, normal human controls.

PD, Parkinson disease humans. 


\subsubsection{Reproducibility and Validity of the Survey Instrument}

On average, studies of reproducibility of the questionnaire revealed a correlation of 0.71 and a kappa score of 0.58 (Table 4-10). This indicates average correlation and moderate agreement in regards to answers supplied on an initial questionnaire and the same questionnaire distributed 6 months later. Based upon a simple one-month food diary, validity studies of the questionnaire revealed a mean correlation of 0.76 and a mean kappa score of 0.72 (Table 4-11), indicating average correlation and substantial agreement with current dietary habits. No significant difference was noted within or between reproducibility and validity of the survey instrument.

\subsubsection{Accumulation of TIQ Derivatives in Rat Brain Over Time}

Using enantiomer selective HPLC-EC and LC-MS/MS, the contentrations of TIQ and salsolinol derivatives were determined in cerebral cortex, hippocampus, ventral midbrain, striatum and cerebellum in rats at age 1 day, 14 days, 30 days, 6 months, 1 year, 1.5 years and 2 years. Trends in TIQ derivative concentration by brain region are shown in Figure 4-1. As observed in data presented in chapter 3, TIQ was found at higher concentrations than its methylated and benzylated derivatives (1-methyl-TIQ, $N$ methyl-TIQ and 1-benzyl-TIQ) in all brain regions at virtually all time points.

Furthermroe, TIQ and its methylated and benzylated derivatives were found at lower concentrations than $(R / S) S A L$ and its derivatives at all ages.

Once again, the regional concentrations of TIQ, 1-methyl-TIQ, N-methyl-TIQ, and 1-benzyl-TIQ exhibited minimal variation across the brain. The concentration of these derivatives appears to change very little over time, with roughly $90 \%$ of the total accumulating within the first one month of life. The level of $N$-methylated salsolinol derivatives, however, tended to remain close to that of their parent salsolinol until later in life (1.5 yrs), when the concentration of salsolinol falls and that of the derivatives tend to rise. The concentration of salsolinol and its derivatives experience a fast ascent within the first 6 months of life. This effect was most pronounced in dopaminergic regions of the brain (ventral midbrain and striatum; Figure 4-1C and D). Interestingly, levels of $N$ methyl-NorSAL accumulate much more slowly in early life compared to (R/S)SAL or Nmethyl-(R/S)SAL, and continue to increase throughout life seemingly never reaching a plateau. The total brain concentration of $N$-methyl-NorSAL was detected on the order of $4 \mu \mathrm{M}$ at 2 years of age. The steepest area of accumulation appears to be from 6 months to 1 year of life within dopaminergic regions. Overall, the net level of TIQ and salsolinol derivatives rises and remains relatively constant over the life of the rat, with the exception of $N$-methyl-NorSAL (Figure 4-1F).

\subsection{DISCUSSION}

Using liquid chromatographic techniques, we have systematically determined the content of several potential neurotoxic TIQ derivatives in 40 of the most commonly consumed plant products in the US. TIQ derivatives were detected in all foods subjected to analysis, revealing particularly high concentrations in banana, cherry, peach, grapefruit, avocado, button mushroom, leaf lettuce and celery. Hydroxylated and 
Table 4-10. Reproducibility of food items.

\begin{tabular}{cccccc}
\hline Vegetable & Spearman Correlation & Kappa* & Fruit & Spearman Correlation Kappa* \\
\hline Potato & 0.92 & 0.86 & Banana & 0.90 & 0.91 \\
Iceberg Lettuce & 0.87 & 0.79 & Apple & 0.92 & 0.88 \\
Tomato & 0.83 & 0.82 & Watermelon & 0.88 & 0.79 \\
Onion & 0.79 & 0.76 & Orange & 0.88 & 0.82 \\
Carrot & 0.73 & 0.60 & Cantaloupe & 0.87 & 0.77 \\
Celery & 0.74 & 0.62 & Grape & 0.87 & 0.71 \\
Yellow Corn & 0.91 & 0.87 & Grapefruit & 0.86 & 0.84 \\
Broccoli & 0.82 & 0.60 & Strawberry & 0.85 & 0.73 \\
Green Cabbage & 0.81 & 0.71 & Peach & 0.85 & 0.77 \\
Cucumber & 0.79 & 0.79 & Pear & 0.79 & 0.71 \\
Bell Pepper & 0.76 & 0.60 & Nectarine & 0.77 & 0.64 \\
Cauliflower & 0.54 & 0.47 & Honeydew Melon & 0.73 & 0.64 \\
Leaf Lettuce & 0.87 & 0.75 & Plum & 0.72 & 0.64 \\
Sweet Potato & 0.72 & 0.81 & Avocado & 0.43 & 0.41 \\
Mushroom & 0.55 & 0.47 & Lemon & 0.79 & 0.66 \\
Green Onion & 0.69 & 0.55 & Pineapple & 0.69 & 0.62 \\
Green Bean & 0.82 & 0.67 & Tangerine & 0.73 & 0.69 \\
Radish & 0.48 & 0.44 & Cherry & 0.59 & 0.63 \\
Yellow Squash & 0.56 & 0.38 & Kiwi Fruit & 0.46 & 0.44 \\
Asparagus & 0.56 & 0.47 & Lime & 0.92 & 0.88 \\
\hline
\end{tabular}

* Kappa interpretation:

$0.00-0.20$ Slight agreement;

$0.21-0.40$ Fair agreement;

$0.41-0.60$ Moderate agreement;

$0.61-0.80$ Substantial agreement;

$0.81-1.00$ Very good agreement. 
Table 4-11. Validity of food items.

\begin{tabular}{cccccc}
\hline Vegetable & Spearman Correlation & Kappa* & Fruit & Spearman Correlation Kappa* \\
\hline Potato & 0.73 & 0.64 & Banana & 0.87 & 0.90 \\
Iceberg Lettuce & 0.72 & 0.64 & Apple & 0.86 & 0.84 \\
Tomato & 0.84 & 0.83 & Watermelon & 0.85 & 0.73 \\
Onion & 0.79 & 0.66 & Orange & 0.85 & 0.77 \\
Carrot & 0.69 & 0.62 & Cantaloupe & 0.79 & 0.71 \\
Celery & 0.73 & 0.69 & Grape & 0.77 & 0.64 \\
Yellow Corn & 0.59 & 0.63 & Grapefruit & 0.54 & 0.47 \\
Broccoli & 0.65 & 0.72 & Strawberry & 0.87 & 0.75 \\
Green Cabbage & 0.97 & 0.93 & Peach & 0.72 & 0.81 \\
Cucumber & 0.92 & 0.88 & Pear & 0.55 & 0.47 \\
Bell Pepper & 0.88 & 0.79 & Nectarine & 0.69 & 0.55 \\
Cauliflower & 0.88 & 0.82 & Honeydew Melon & 0.82 & 0.67 \\
Leaf Lettuce & 0.87 & 0.77 & Plum & 0.83 & 0.82 \\
Sweet Potato & 0.87 & 0.71 & Avocado & 0.79 & 0.76 \\
Mushroom & 0.86 & 0.84 & Lemon & 0.73 & 0.60 \\
Green Onion & 0.85 & 0.73 & Pineapple & 0.74 & 0.62 \\
Green Bean & 0.85 & 0.77 & Tangerine & 0.91 & 0.87 \\
Radish & 0.79 & 0.71 & Cherry & 0.82 & 0.60 \\
Yellow Squash & 0.75 & 0.68 & Kiwi Fruit & 0.81 & 0.71 \\
Asparagus & 0.65 & 0.76 & Lime & 0.79 & 0.79 \\
\hline
\end{tabular}

* Kappa interpretation:

$0.00-0.20$ Slight agreement;

$0.21-0.40$ Fair agreement;

$0.41-0.60$ Moderate agreement;

$0.61-0.80$ Substantial agreement;

$0.81-1.00$ Very good agreement. 

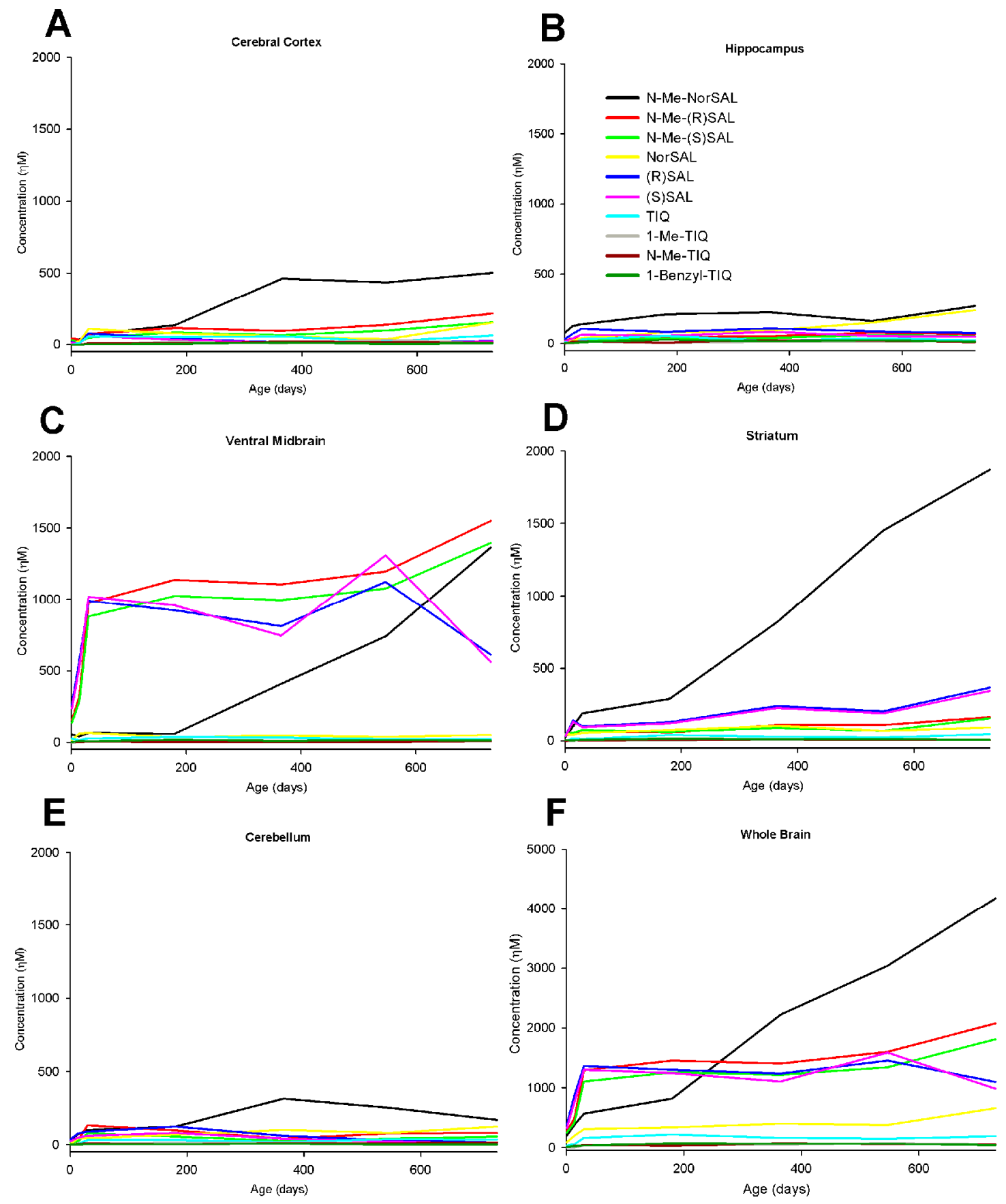

Figure 4-1. TIQ derivative accumulation in the rat brain over time.

The contrations of TIQ derivatives from (A) frontal cerebral cortex, (B) hippocampus, (C) ventral midbrain, $(D)$ striatum and $(E)$ cerebellum were determined in rat brain using HPLC-EC and LC-MS/MS over time. Total brain concentrations are depicted in F. 
methylated derivatives of TIQ were present at high concentrations relative to TIQ, as were the $N$-methylated derivatives of salsolinol. Enantiomeric salsolinol derivatives were detected as racemic mixtures in all plants, with no evidence of stereoselective enzymatic synthesis. The mushroom family (Agaricaceae) contained the highest average TIQ derivative load, salsolinol derivative load and total toxin load of all plant families. The banana family (Musaceae), rose family (Rosaceae), sunflower family (Asteraceae) and pineapple family (Bromeliaceae) also contained particularly high total average neurotoxin loads.

The first reports of TIQ derivative detection in foods utilized GC-MS techniques to quantify TIQ and 1-methyl-TIQ in white wine $(1.7 \mathrm{ng} / \mathrm{g}$ and $354 \mathrm{ng} / \mathrm{g}$, respectively), cheese $(1.7 \mathrm{ng} / \mathrm{g}$ and $354 \mathrm{ng} / \mathrm{g}$, respectively) and cocoa $(1.1 \mathrm{ng} / \mathrm{g}$ and $12 \mathrm{ng} / \mathrm{g}$, respectively) (Makino et al 1988; Makino et al 1990). Niwa and co-workers later detected TIQ in meat (0.9 to $1.8 \mathrm{ng} / \mathrm{g})$, cheese $(5.2 \mathrm{ng} / \mathrm{g})$, banana $(2.2 \mathrm{ng} / \mathrm{g})$, wine $(0.6$ $\mathrm{ng} / \mathrm{g})$, beer $(0.4 \mathrm{ng} / \mathrm{g})$ and whiskey $(0.7 \mathrm{ng} / \mathrm{g})$ using GC-MS and concluded that TIQ concentrations were too low in foods to be revelant in PD (Niwa et al 1989). Finally, using enantiomer selective HPLC-EC, Deng and colleagues detected $(R)$ and $(S) S A L$ in dried banana ( $65.34 \mathrm{ng} / \mathrm{g}$ and $61.63 \mathrm{ng} / \mathrm{g}$, respectively), "German wine" (6.18 ng/g and $7.85 \mathrm{ng} / \mathrm{g}$, respectively) and "French wine" (3.32 ng/g and $4.93 \mathrm{ng} / \mathrm{g}$, respectively) (Deng et al 1997). N-methyl-(R/S)SAL was not detected. While tempting, direct comparison between the results of these studies and those of the present study becomes difficult when the ambiquity of sample ages, orgins and sizes in the abovementioned studies are taken into account. Not to mention the sample derivitization and SPE involved in preparation for GC-MS, as detailed in chapter 3 . With this in mind, our observed detection of TIQ in fresh banana at $984 \mathrm{ng} / \mathrm{g}$ certainly does not compare with that of $2.2 \mathrm{ng} / \mathrm{g}$ in a single $20 \mathrm{~g}$ sample of unknown banana (Niwa et al 1989). However, consistent with our findings in banana, Deng and colleagues detected $(R / S) S A L$ as a racemic mixture using HPLC-EC (Deng et al 1997).

In aggregate, the present results of TIQ derivatives in plants suggest several conclusions: (1) that TIQ derivatives are readily formed within plants as suggested by Collins and co-workers (Collins 2002); (2) that derivatives of TIQ and salsolinol are formed via methylation or oxidation reactions, rather than de novo; (3) that amino acid/aldehyde condensation is the predominant synthetic pathway for $(R / S) S A L$, with very little to no input from enzymatic synthesis as found in dopaminergic regions of mammalian brain; (4) that plant families tend to have similar TIQ derivative profiles and overall content, suggesting a genetic component to isoquinoline and $\beta$-carboline synthesis; and (5) that several TIQ derivatives known to cross the blood-brain barrier (TIQ, 1-methyl-TIQ, 1-benzyl-TIQ and N-methyl-NorSAL) are found at concentrations high enough for dietary accumulation in brain to be a feasible hypothesis.

Utilizing a lifetime food-item intake questionnaire, our results demonstrate a trend of increased intake of several plants products (bananas, apples, watermelon, oranges, cantaloupe, grapes, grapefruit, potatoes, iceberg lettuce, tomatoes, onions, carrots and celery) in PD patients relative to age-matched controls. However, only the lifetime intake of oranges reached statistical significance. Interestingly, members of the rue (Rutaceae), banana (Musaceae), gourd (Cucurbitaceae), carrot (Apiaceae) and nightshade (Solanaceae) families are well represented in this list. For instance, bananas are especially rich in both TIQ and salsolinol derivatives, particularly TIQ, 1-benzyl-TIQ, $(R / S) S A L$ and $N$-methyl-NorSAL. Members of the rue family (orange and grapefruit) 
have particularly high concentrations of salsolinol derivatives such as $(R / S) S A L$ and $N$ methyl-(R/S)SAL. Apples also appear to be rich in $N$-methyl-NorSAL. Celery and carrot, members of the Apiaceae family, also concentrate $(R / S) S A L$ and $N$-methyl-NorSAL. Potato and tomato, in contrast, do not seem to concentrate any particular TIQ derivative per se, but contain modest concentrations of many derivatives. Thus, many of the food items with a tendency for heaver consumption by PD patients over their lifetime were found to concentrate one or several potentially neurotoxic TIQ derivatives.

The risk of PD has been previously associated the intake of a small subset of whole food items and their nutrients or components. For example, decreased potato and tree nut intake, icreased carbohydrate intake, increased iron intake or decreased intake of niacin and antoxidants (such as vitamine $\mathrm{E}$ ) have been shown to be weakly associated with the development of PD (Anderson et al 1999; Davies et al 1994; Fall et al 1999; Golbe et al 1988; Hellenbrand et al 1996a; Hellenbrand et al 1996b; Johnson et al 1999; Logroscino et al 1996; Logroscino et al 1998). In comparison with traditional single-food or nutrient methods, the dietary pattern approach to analysis is a more appropriate measure for several reasons. In reality, humans eat combinations of food and its constituents (nutrients, toxins, etc.), which leads to a high collinearity among many of these compounds and foods. It is therefore difficult to identify the effect of a single food or substance on a disease state. Linear transformation using principal component analysis therefore represents a combination of food and their components and may be a more powerful predictor of health outcomes than a single measure alone (Hu 2002). Furthermore, a dietary pattern analysis offers an approach for better understanding the complexities of eating behaviors of different population groups and subgroups and may be used to effectively develop dietary intervention strategies (Gao et al 2006; Millen et al 1996). Ultimately, the development of idiopathic PD is multifactorial and no single aspect alone (such as consumption of a specific food item or substance) will likely ever demonstrate a direct cause-effect relationship in such a diverse background of risk factors associated with disease.

Using common methods of analysis, the results of reproducibility and validity studies may be considered modest and on-par with several other dietary questionnaires (Block \& Hartman 1989; Buzzard et al 2001; Matthys et al 2007; Nelson \& Lytle 2009). However, due of the retrospective design of the survey instrument, our results are very likely to be affected by recall or selection bias to some extent. For instance, our validity study is only revelant to the current decade of life and therefore cannot approximate the suitability of the instrument for any other years. Furthermore, we could not and did not correct for any other known PD risk factors and thus cannot exclude the possibility of residual confounding by other unknown factors. Finally, we cannot exclude the possibility that consumption of fruits and vegetables contaminated with pesticides or polychlorinated biphenyls may contribute to the observed results. For instance, several of these compounds have been shown to have selective toxicity for nigrastriatal neurons in animal models (Mariussen \& Fonnum 2001).

The present study is also the first to demonstrate the accumulation of TIQ derivatives over time in rat brain. Interestingly, the concentration of TIQ derivatives appears to change very little over time, with high accumulation within the first one month of life. In contrast, salsolinol derivatives experience a fast ascent within the first 6 months of life within dopaminergic regions, followed by another rise in concentration around $1.5 \mathrm{yrs}$ in age. Levels of $N$-methyl-NorSAL accumulate more slowly in early life, followed by rapid accumulation around 6 months to 1 year of age with continued 
increases throughout life. Overall, the net content of TIQ and salsolinol derivatives rises early and remains relatively constant over the life of the rat, with the exception of $N$ methyl-NorSAL. Interestingly, levels of dopamine and dopamine metabolites (DOPAC and HVA) rise throughout life in the ventral midbrain and striatum, and in effect mirror the rise in $N$-methyl-NorSAL (data not shown). For instance, in the striatum DOPAC levels at 2 years reached $6 \mu \mathrm{M}$, while that of $N$-methyl-NorSAL was $1.8 \mu \mathrm{M}$. Mean total brain content of DOPAC and HVA at 2 years of age was $8 \mu \mathrm{M}$ and $1.5 \mu \mathrm{M}$, respectively. Mean total brain $N$-methyl-NorSAL content was determined to be $4 \mu \mathrm{M}$. For reference, total brain $N$-methyl-(R)SAL and $N$-methyl-(S)SAL content was $2 \mu \mathrm{M}$ and $1.5 \mu \mathrm{M}$, respectively at the 2 year time point. The following conclusions can be drawn from these results: (1) TIQ derivatives can and do accumulate within regions of the rat brain from endogenous and/or exogenous sources; (2) metabolism of TIQ derivatives in rat brain appears to be either stable without net change in content or non-existant with gradual accumulation over time; and (3) the idea of salsolinol derivatives as metabolites of dopamine is strengthened, as their concentrations appear to mirror rises in dopamine content and to be intermediate to those of DOPAC and HVA. Similar studies in human brain regions over time are necessary for extrapolation of these conclusions to humans and their implications for neurodegenerative disease.

\subsection{CONCLUSIONS}

Epidemiological studies suggest that environmental factors contribute to the pathogenesis of PD. The discovery of MPTP confirmed that dopaminergic cell death could indeed be precipitated by environmental neurotoxins. The TIQs are structurally similar to MPTP, inhibit complex I of the respiratory chain, and can initiate apoptosis in cultured dopaminergic neurons. Using enantiomeric-selective HPLC-EC and LCMS/MS, the concentrations several TIQ derivatives were determined in those fruits $(N=$ $20)$ and vegetables $(N=20)$ most commonly consumed in the US. TIQ derivatives were detected in all foods subjected to analysis, revealing particularly high concentrations in banana, cherry, peach, grapefruit, avocado, button mushroom, leaf lettuce and celery. Enantiomeric salsolinol derivatives were detected as racemic mixtures in all plants, with no evidence of stereoselective enzymatic synthesis. The mushroom family (Agaricaceae) contained the highest average TIQ derivative load, salsolinol derivative load and total toxin load of all plant families. The banana family (Musaceae), rose family (Rosaceae), sunflower family (Asteraceae) and pineapple family (Bromeliaceae) also contained particularly high total average neurotoxin loads. Utilizing a lifetime food-item intake questionnaire, we demonstrate a trend of increased intake of several plants products (bananas, apples, watermelon, oranges, cantaloupe, grapes, grapefruit, potatoes, iceberg lettuce, tomatoes, onions, carrots and celery) in PD patients. This finding was most significant for oranges. These items include members of the rue (Rutaceae), banana (Musaceae), gourd (Cucurbitaceae), carrot (Apiaceae) and nightshade (Solanaceae) families, found to concentrate several potentially neurotoxic TIQ derivatives. Finally, we demonstrate the time-dependent accumulation of TIQ derivatives in rat brain. The concentration of TIQ derivatives appears to change very little over time, with high accumulation within the first one month of life. Salsolinol derivatives experience a more rapid ascent within the first 6 months of life within dopaminergic regions, followed by another concentration increase later in life. Levels of $N$-methyl-NorSAL accumulate more slowly, followed by rapid accumulation around 6 months with sustained increases. Overall, the net content of TIQ and salsolinol derivatives rises early and remains relatively constant over the life of the rat, with the 
exception of $\mathrm{N}$-methyl-NorSAL. This data demonstrates the presence of potentially toxic TIQ derivatives in plants commonly utilized as food sources and provides evidence to support the accumulation of TIQs in the rodent brain over time. Taken together, this may provide a link between dietary risk factors, the accumulation of toxic TIQ derivatives in the brain and the pathogenesis of PD. 


\section{CHAPTER 5. IN VITRO TOXICITY OF METHYLATED TETRAHYDROSIOQUINOLINE DERIVATIVES}

\section{$5.1 \quad$ INTRODUCTION}

A significant amount of research has been dedicated to the precise cause of nigral cell death in idiopathic PD. One leading hypothesis is oxidative cell injury caused by mitochondrial respiratory failure with a defect in NADH-ubiquinone oxidoreductase (complex I, EC 1.6.5.3) (Mizuno et al 1998). In support of this hypothesis, complex I activities in multiple brain regions of PD patients have been shown to be decreased relative to controls (Parker et al 1989; Schapira et al 1990). Furthermore, of the potential cellular targets of MPP+, mitochondrial complex I is believed to be its most potent site of action (Tipton \& Singer 1993). Complex I is an extremely large and intricate enzyme system consisting of 41 different subunits in mammals. Functionally, complex I serves as the entry point for electrons into the ETC and is located within the inner mitochondrial membrane, where it catalyzes electron transfer from NADH, generated from the tricarboxylic acid (TCA) cycle in the mitochondrial matrix, to ubiquinone (Lardy \& Ferguson 1969). Although some reports have suggested that a genetic defect in mitochondrial DNA causes complex I deficiency in familial PD patients, sporadic PD cannot be explained in terms of genetic predisposition alone, and unknown endogenous or exogenous factors contributing to complex I inhibition are a revelant possibility (Kotake \& Ohta 2003).

Salsolinol, an enantiomeric 6,7-dihydroxylated TIQ derivative formed by condensation of dopamine and formaldehyde, is found at higher concentrations within dopaminergic areas of the human brain and has been shown to inhibit complex 1 of the ETC (Musshoff et al 2003; Musshoff et al 2005; Musshoff et al 2000; Musshoff et al 1999; Shavali et al 2003; Wanpen et al 2004). Furthermore, $(R / S)$ salsolinol and its $N$ methylated derivatives have also been detected in the brain, CSF and urine of PD patients (Mravec 2006). In addition to their ability to cross the blood-brain barrier, there is also evidence that $N$-methyl-6,7-dihydroxylated TIQs such as $N$-methyl-(R/S)salsolinol and $N$-methyl-norsalsolinol are more toxic than their parent salsolinol (Maruyama et al 1993; Storch et al 2002). These reports suggest that methylation at the $N$-position confers heightented toxicity and that these compounds could act as endogenous neurotoxins that contribute to the death of dopaminergic neurons. However, little is known about their relative toxicity and the mechanism by which these compounds induce cell death.

There is also data to suggest that another methylated TIQ derivative, 1-methylTIQ, may prevent the neurotoxic effects of MPP+ (Makino et al 1990; Parrado et al 2000; Yamakawa et al 1999). However, a protective effect of 1-methyl-TIQ may not be an accurate description, and instead may be due to a shielding effect of complex I from the much more potent toxin MPP+ (Parrado et al 2000). For instance, a recent study of the cytotoxicity of several TIQ derivatives in dopaminergic SH-SY5Y cells demonstrated that 1-methyl-TIQ exhibits cytotoxicity and complex I inhibition on par with other non-polar TIQ derivatives (Kotake et al 2007). The possibility of a neuroprotective TIQ derivative is intriguing, but conflicting reports of its toxicity are troublesome and a more detailed account of its cellular activity is required before any definitive assertions can be made. 
In the present study, human SH-SY5Y cells were utilized to quantify the reduction in mitochondrial membrane potential and resultant cytotoxicity of three methylated TIQ derivatives, $N$-methyl-norsalsolinol, $N$-methyl- $(R / S)$ salsolinol and 1 methyl-TIQ. The human neuroblastoma cell line, SH-SY5Y, is a model for dopaminergic neurons. These cells express several markers of dopaminergic neurons including $\mathrm{TH}$, the vesicular monoamine transporter (VMAT), the DAT and $\alpha$-synuclein (Fountaine \& Wade-Martins 2007; Takahashi et al 1994). SH-SY5Y cells have also been widely used to study apoptosis of dopaminergic neurons by parkinsonism-inducing neurotoxins (Ouyang \& Shen 2006; Valverde et al 2008). Here, we quantify the direct toxicity of methylated TIQ derivatives by a reduction in overall cellular metabolism, a decrease in mitochondrial membrane potential and changes in nuclear morphology consistent with apoptosis. We demonstrate that all three methylated derivatives destroy dopaminergic neurons in a dose-dependent manner. Additionally, physical properties emparted by overall compound charge and structure are reported and related to membrane permeability and cytotoxicity.

\subsection{MATERIALS AND METHODS}

\subsubsection{Chemicals}

Butanol, formaldehyde, dimethyl sulfoxide (DMSO), rotenone, sodium acetate and sodium cyanoborohydride were purchased from Sigma-Aldrich (St. Louis, MO, USA). (R/S)saslolinol and norsalsolinol were purchased from Acros Organics (Geel, Belgium). 1-methyl-TIQ was purchased from AsisChem Inc. (Cambridge, MA, USA).

\subsubsection{Synthesis of $N$-methyl-norsalsolinol and $N$-methyl-(R/S)salsolinol}

The tertiary amines $N$-methyl-norsalsolinol and $N$-methyl-(R/S)salsolinol were prepared from their respective secondary amines norsalsolinol and $(R / S)$ salsolinol by reductive methylation in the presence of formaldehyde and sodium cyanoborohydride. The reaction mixtures contained $0.5 \mathrm{M}$ of the amine, $2.5 \mathrm{M}$ formaldehyde, $0.2 \mathrm{M}$ sodium acetate, and $1 \mathrm{M}$ sodium cyanoborohydride at $\mathrm{pH} 5$. The reaction mixture was incubated at room temperature overnight under nitrogen in the dark. The sample was clarified by centrifugation and the clear solution was acidified with $\mathrm{HCl}$ to $\mathrm{pH} 0.1$ to decompose unreacted cyanoborohydride. The solvent was evaporated and the product was extracted from the residue with isopropyl alcohol. Evaporation of the solvent and extraction of the residue was repeated twice. The final product was stored as a solution in isopropyl alcohol at $-20^{\circ} \mathrm{C}$.

\subsubsection{SH-SY5Y Cell Culture}

The human neuroblastoma cell line, SH-SY5Y, was obtained from the American Type Culture Collection (Manassas, VA, USA). SH-SY5Y cells were grown in minimum essential medium: F-12 nutrient mixture (1:1) containing $15 \%$ fetal bovine serum and $1 \%$ non-essential amino acids (Gibco, Rockville, MD, USA) at $37^{\circ} \mathrm{C}$ under $5 \% \mathrm{CO}_{2}$ in a humidified atmosphere. 


\subsubsection{Compound Handling and Dilution}

All compounds were stored in anhydrous form under vaccum at $-80^{\circ} \mathrm{C}$ until the time of administration. Stock solutions $(1 \mathrm{mM})$ of all compounds were prepared in a laminar flow ventilation hood using DMSO and then diluted in culture medium to final concentrations. DMSO concentration in the culture medium did not exceed $0.01 \%$. For each treatment, fresh solutions were used to avoid any possible breakdown by storage.

\subsubsection{MTT Assay}

Cell viability was measured using the MTT assay (Roche Applied Science, Indianapolis, IN, USA) (Molina-Jimenez et al 2003). This colorimetric assay measures the metabolic activity of viable cells based on cleavage of the tetrazolium salt WST-1 to water-soluble formazan by mitochondrial dehydrogenase in living cells. SH-SY5Y cells $\left(5 \times 10^{3}\right.$ cells/well) were seeded in 96 -well plates and incubated with $N$-methylnorsalsolinol, $N$-methyl- $(R / S)$ salsolinol or 1 -methyl-TIQ at concentrations of $1 \mu \mathrm{M}, 10$ $\mu \mathrm{M}, 20 \mu \mathrm{M}, 50 \mu \mathrm{M}, 200 \mu \mathrm{M}$ and $500 \mu \mathrm{M}$ at $37^{\circ} \mathrm{C}$ for 24 or $48 \mathrm{hrs}$. Rotentone at concentrations of $0.01 \mu \mathrm{M}, 0.1 \mu \mathrm{M}, 1 \mu \mathrm{M}, 5 \mu \mathrm{M}$ and $20 \mu \mathrm{M}$ was used as a positive control. After incubation, the medium was removed and $100 \mu \mathrm{l}$ of MTT reagent $(0.5$ $\mathrm{mg} / \mathrm{ml}$ MTT in complete medium) was added to each well. Following an additional $4 \mathrm{hrs}$ of incubation at $37^{\circ} \mathrm{C}, 100 \mu \mathrm{l}$ of DMSO was added to dissolve the formazan crystals, and the absorbance was then measured at 550 and $690 \mathrm{~nm}$ using a microplate reader (Bio-Rad Laboratories, Hercules, CA, USA). Wells without cells were used as blanks and were subtracted as background from each sample. Wells with cells but without toxins were used as negative control. LC $_{50}$ values were calculated with absorbance data using non-linear regression analysis.

\subsubsection{Measurement of Mitochondrial Membrane Potential $\left(\Delta \Psi_{m}\right)$}

The collapse of the electrochemical gradient across the mitochondrial membrane is an early event in cellular apoptosis. JC-1 dye localizes to the inner mitochondrial membrane where it forms either monomers or aggregates based on the mitochondrial membrane potential $\left(\Delta \Psi_{m}\right)$. At high $\Delta \Psi_{m}$ (healthy cells), JC-1 accumulates sufficiently in the mitochondria to form aggregates (J-aggregates) that exhibit red fluorescence. Conversely, at lower $\Delta \Psi_{m}$ (dying cells) less dye enters mitochondria resulting in monomers that demonstrate green fluorescence withing the cell cytoplasm. The JC-1 aggregate:monomer ratio has been used as a tool to estimate changes in $\Delta \Psi_{m}$ (White \& Reynolds 1996). SH-SY5Y cells $\left(5 \times 10^{3}\right.$ cells/well) were seeded in 8-well glass slides and incubated with $N$-methyl-norsalsolinol, $N$-methyl-(R/S)salsolinol or 1-methyl-TIQ at concentrations of $100 \mu \mathrm{M}$ at $37^{\circ} \mathrm{C}$ for $15 \mathrm{~min}, 30 \mathrm{~min}$ and $1 \mathrm{hr}$. Rotenone at a concentration of $1 \mu \mathrm{M}$ was utilized as a positive control. JC-1 was dissolved in DMSO and further diluted in minimal essential medium ( $1 \mathrm{mg} / \mathrm{ml}$ final concentration). After removal of the toxin-containing medium, cells were loaded with $\mathrm{JC}-1$ for 15 min at $37^{\circ} \mathrm{C}$, rinsed twice with phosphate buffered saline and immediately photographed on a Leica epifluorescent microscope using a rhodamine filter set (Leica Microsystems, Bannockburn, IL, USA). Ten photos were taken randomly from each well. The $\mathrm{red} / \mathrm{green}$ ratio was determined densitometrically using ImageJ software (US National 
Institutes of Health, Bethesda, MD, USA, http://rsb.info.nih.gov/ij/) from randomly selected micrographs in each experiment (10 photos per well). Reported values represent percentages normalized to control cells.

\subsubsection{DAPI Histochemistry}

To assess nuclear morphology and apoptosis, SH-SY5Y cells were stained with 4,6-diamidino-2-phenylindole (DAPI; Sigma-Aldrich, St. Louis, MO, USA), a fluorescent compound that binds to double-stranded DNA. SH-SY5Y cells $\left(5 \times 10^{3}\right.$ cells/well) were seeded in 8-well glass slides and incubated with $N$-methyl-norsalsolinol, $N$-methyl$(R / S)$ salsolinol or 1 -methyl-TIQ at concentrations of $100 \mu \mathrm{M}$ at $37^{\circ} \mathrm{C}$ for $1 \mathrm{hr}$. Rotenone at a concentration of $1 \mu \mathrm{M}$ was utilized as a positive control. After exposure, cells were fixed in phosphate buffered saline containing $3.7 \%$ paraformaldehyde for $15 \mathrm{~min}$. After fixation, cells were washed twice with PBS for $5 \mathrm{~min}$ and then treated with DAPI (1 $\mathrm{mg} / \mathrm{ml}$ ) in PBS. After three 5 min wash cycles, slides were coverslipped with 1,3 diethyl8-phenylxanthine mounting compound (DPX; Sigma-Aldrich, St. Louis, MO, USA) and visualized under an epifluorescence microscope with ultraviolet excitation at $300-500 \mathrm{~nm}$ (Leica Microsystems, Bannockburn, IL, USA).

\subsubsection{Calculation of Molecular Properties}

The octanol/water partition coefficient (Log P), topological polar surface area (TPSA) and total molecular volume $\left(V_{m}\right)$ were calculated for each compound using Mitools Toolkit software (http://www.molinspiration.com).

\subsubsection{Statistical Analyses}

Statistical analyses were based on median values derived from technical triplicates. The results of experiments at the $24 \mathrm{hr}$ time point were analyzed by means of one-way ANOVA using SAS software version 9.0 (SAS Institute Inc., Cary, NC, USA) for comparisons between compounds at each concentration. The results of experiments at the $48 \mathrm{hr}$ time point were analyzed by means of a student's $t$ test for comparisons of time ( $24 \mathrm{vs} 48 \mathrm{hrs}$ ) for each concentration. An alpha ( $\alpha$ ) of 0.05 was chosen for statistical significance.

\subsection{RESULTS}

\subsubsection{Cytotoxicity of TIQ Derivatives}

Using the MTT assay to evaluate overall cell viability, SH-SY5Y cells were exposed to either $N$-methyl-(R/S)salsolinol, $N$-methyl-norsalsolinol or 1-methyl-TIQ for 24 or 48 hrs. The pesticide and known mitochondrial complex I inhibitor rotenone served as a positive control. As shown in Figure 5-1, $24 \mathrm{hr}$ treament of SY5Y cells with $N$-methyl-(R/S)salsolinol, $N$-methyl-norsalsolinol and 1-methyl-TIQ resulted in significant cytotoxicity. Comapred to non-treated cells, $N$-methyl-norsalsolinol $\left(\mathrm{LC}_{50}=0.305 \mathrm{mM}\right)$, $N$-methyl- $(R / S)$ salsolinol $\left(\mathrm{LC}_{50}=0.377 \mathrm{mM}\right)$ and 1-methyl-TIQ $\left(\mathrm{LC}_{50}=2.153 \mathrm{mM}\right)$ 

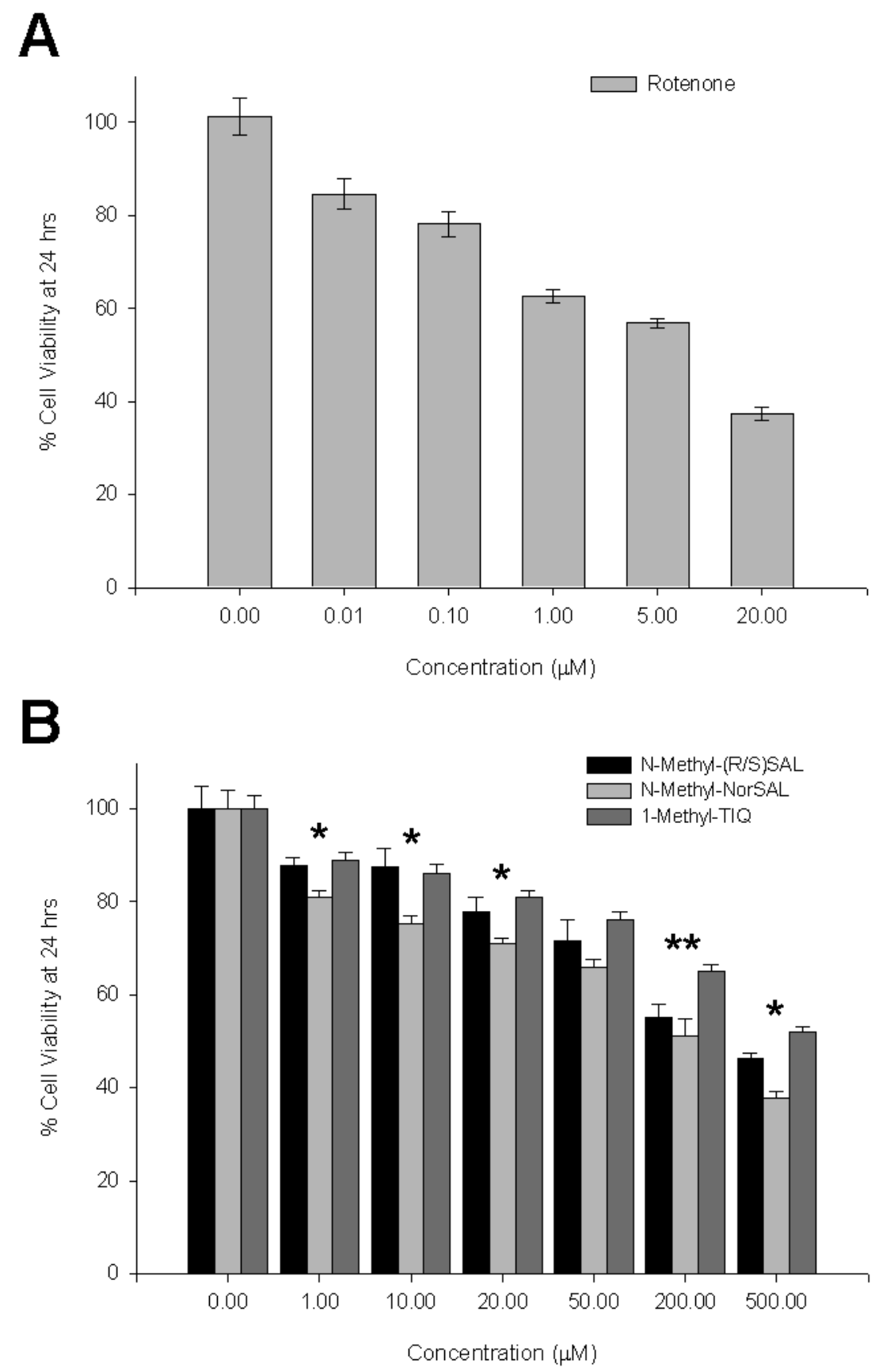

Figure 5-1. Viability of SH-SY5Y cells after 24 hour exposure to rotenone and TIQ derivatives.

(A) Rotenone; (B) N-methyl-(R/S)salsolinol, $N$-methyl-norsalsolinol and 1-methyl-TIQ. * Indicates significant difference between $N$-methyl-norsalsolinol and others $(P<0.05)$.

** Indicates significant difference between 1-methyl-TIQ and others $(P<0.05)$. 
significantly reduced cell viability in a dose-dependent manner (Table 5-1). However, $N$ methyl-norsalsolinol was significantly more cytotoxic at $1 \mu \mathrm{M}(F(2,27)=5.77, P<$ $0.008), 10 \mu \mathrm{M}(F(2,27)=7.27, P<0.002)$ and $500 \mu \mathrm{M}(F=8.29, P<0.0001)$ concentrations relative to $N$-methyl-(R/S)salsolinol and 1-methyl-TIQ. Conversely, a trend of decreased toxicity of 1-methyl-TIQ relative to $N$-methyl- $(R / S)$ salsolinol and $N$ methyl-norsalsolinol was observed, but only reached significance at the $200 \mu \mathrm{M}(F=$ 8.29, $P<0.0001)$ concentration. Overall, $N$-methyl- $(R / S)$ salsolinol and $N$-methylnorsalsolinol exhibited similar toxicity $\left(\mathrm{LC}_{50}=0.376 \mathrm{mM}\right.$ vs $\left.0.305 \mathrm{mM}\right)$, while 1-methlyTIQ was much less potent $\left(\mathrm{LC}_{50}=2.153 \mathrm{mM}\right)$. In aggregate, all methylated TIQ derivatives were much weaker toxins than rotentone $\left(\mathrm{LC}_{50}=0.013 \mathrm{mM}\right)$.

The effect of exposure duration ( $24 \mathrm{vs} 48 \mathrm{hrs}$ ) was most pronounced for rotentone, reaching significance at $1 \mu \mathrm{M}, 5 \mu \mathrm{M}$ and $20 \mu \mathrm{M}$ concentrations (Figure 5-2; $P$ $<0.05)$. In contrast, exposure duration in methylated TIQ derivatives was only evident at higher concentrations, reaching significance at $500 \mu \mathrm{M}$ for $N$-methyl-(R/S)salsolinol, and at $200 \mu \mathrm{M}$ and $500 \mu \mathrm{M}$ for $N$-methyl-norsalsolinol $(P<0.05)$.

\subsubsection{Decreased $\Delta \Psi_{\mathrm{m}}$ after Acute Exposure to Methylated TIQ Derivatives}

The JC-1 dye was utilized to evaluate the $\Delta \Psi_{\mathrm{m}}$ induced by exposure to TIQ derivatives and rotenone. As shown in Figure 5-3, $N$-methyl-(R/S)salsolinol, $N$-methylnorsalsolinol and 1-methyl-TIQ all significantly decreased the red/green fluorescence ratio, and therefore decreased $\Delta \Psi_{\mathrm{m}}$, after $15 \mathrm{~min}$ exposure relative to untreated SY5Y cells $(P<0.05)$. The decrease in $\Delta \Psi_{\mathrm{m}}$ was time dependent, as the $\mathrm{JC}-1 \mathrm{red} / \mathrm{green}$ fluorescence ratio continued to decrease at the $30 \mathrm{~min}$ and $1 \mathrm{hr}$ exposure time points for all compounds (Figure 5-4). Emulating the results of cell viability experiments, the timedependent decrease in $\Delta \Psi_{\mathrm{m}}$ was most pronounced for rotenone. $N$-methyl$(R / S)$ salsolinol and $N$-methyl-norsalsolinol exhibited almost identical changes in $\Delta \Psi_{\mathrm{m}}$, while 1-methyl-TIQ demonstrated the weakest decrease in $\Delta \Psi_{\mathrm{m}}$ over time.

\subsubsection{Nuclear Morphology after Exposure to TIQ Derivatives}

Utilizing the DAPI staining protocol, nuclear morphology was evaluated after exposure to $N$-methyl-(R/S)salsolinol, $N$-methyl-norsalsolinol and 1-methyl-TIQ for $1 \mathrm{hr}$. As shown in Figure 5-5, typical apoptotic morphology such as chromatin condensation, nuclear fragmentation and pyknotic cellular contraction are visible after treatment with all methylated TIQ derivatives. Once again, the most significant histochemical findings consistent with apoptosis were observed after exposure to rotenone.

\subsubsection{Molecular Properties of TIQ Derivatives}

The calculated molecular properties of $N$-methyl- $(R / S)$ salsolinol, $N$-methylnorsalsolinol, 1-methyl-TIQ and rotenone are listed in Table 5-1 (a complete listing of molecular properties of all TIQ derivatives found in this document can be found in Appendix A, Table A-2). The $\log P$, a standard measure of solubility, was highest for rotenone and 1-methyl-TIQ (3.585 and 2.048, respectively). A high log $P$ value indicates a predilection for sequestration in non-polar or hydrophobic environments. As a direct 
Table 5-1. Cytotoxicity and physical properties of study compounds.

\begin{tabular}{lcccc}
\hline Compound & $\mathrm{LC}_{50} 24 \mathrm{hrs}^{*}(\mathrm{mM})$ & Log P & TPSA $\left(\AA^{2}\right)$ & $V_{\mathrm{m}}\left(\mathrm{cm}^{3} / \mathrm{mol}\right)$ \\
\hline Rotenone & 0.013 & 3.585 & 63.241 & 348.335 \\
$N$-Methyl-Norsalsolinol & 0.305 & 0.741 & 43.694 & 168.988 \\
$N$-Methyl- $(R / S)$ Salsolinol & 0.377 & 1.301 & 43.694 & 185.575 \\
1-Methyl-TIQ & 2.153 & 2.048 & 12.027 & 152.596 \\
\hline
\end{tabular}

* $\mathrm{LC}_{50} 24 \mathrm{hrs}=$ Median Lethal Concentration at $24 \mathrm{hrs}$
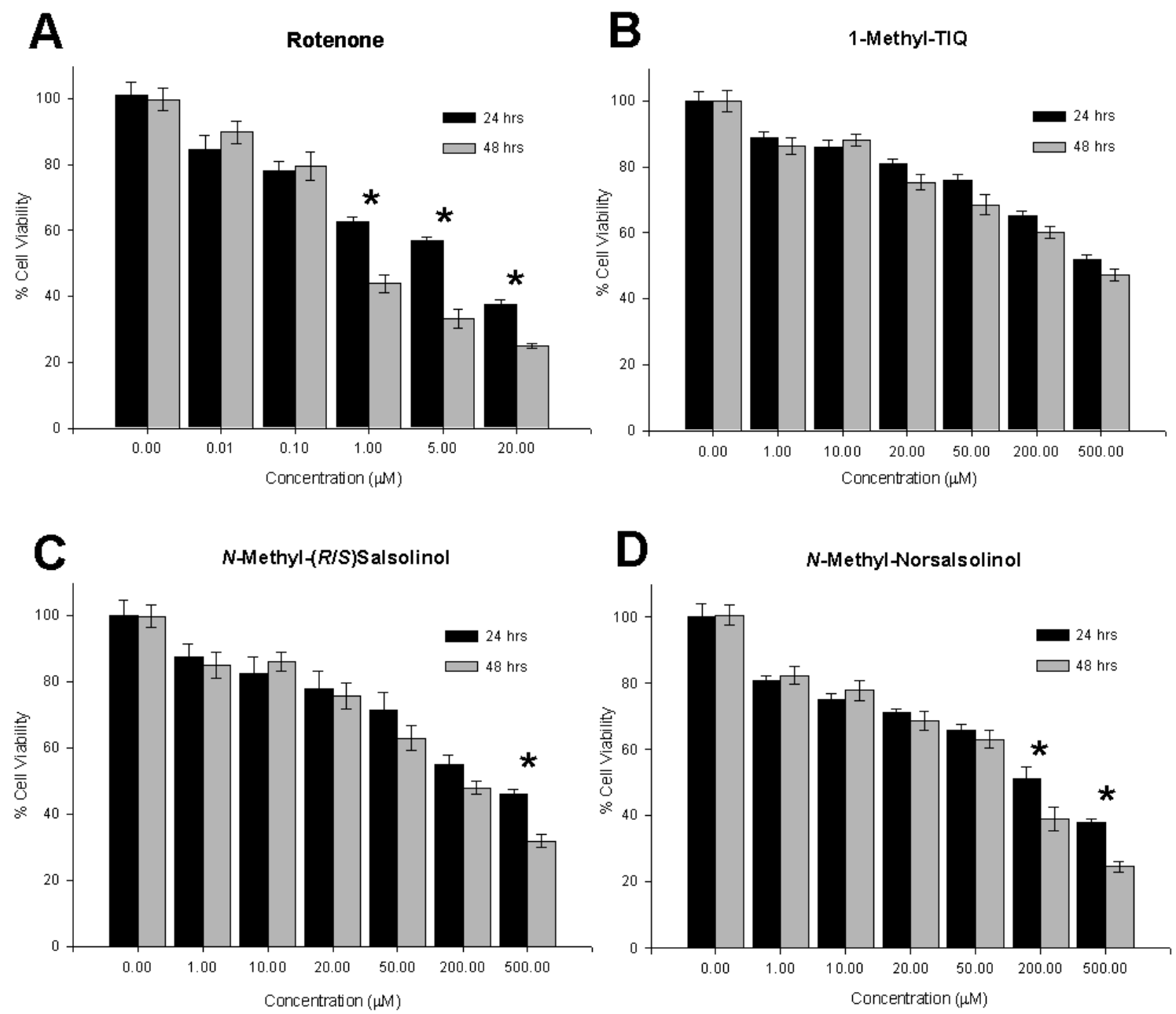

Figure 5-2. Viability of SH-SY5Y cells after 48 hour exposure to rotenone and TIQ derivatives.

(A) Rotenone; (B) 1-methyl-TIQ; (C) N-methyl-(R/S)salsolinol; (D) N-methylnorsalsolinol.

* Indicates a significant difference between the 24 and $48 \mathrm{hr}$ exposure period $(P<0.05)$. 


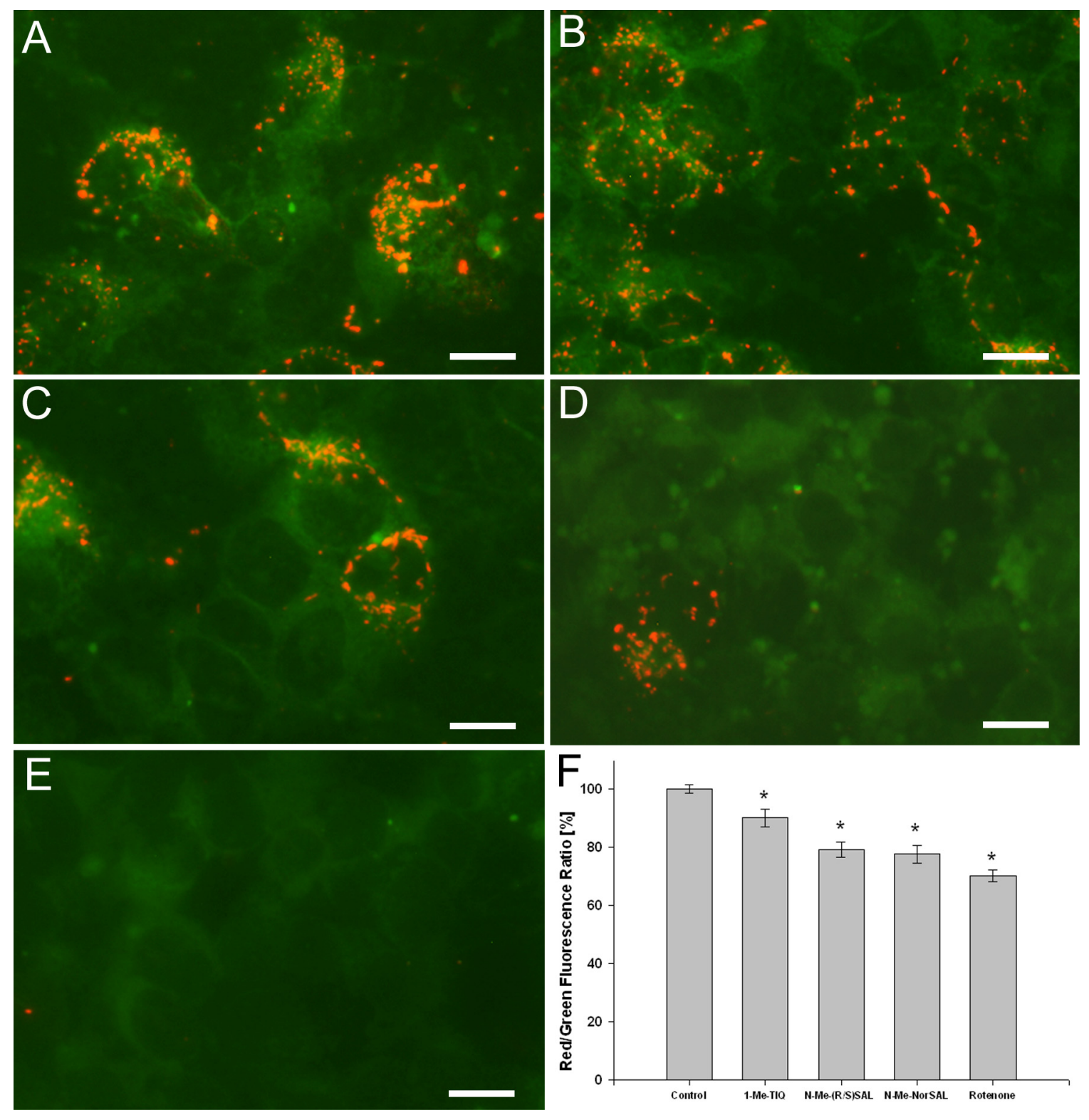

Figure 5-3. Decreased mitochondrial membrane potential $\left(\Delta \Psi_{\mathrm{m}}\right)$ after acute exposure to TIQ derivatives.

JC-1 staining of SH-SY5Y cells after 15 min exposure to mitochondrial toxins. (A) Compound-free control; (B) $100 \mu \mathrm{M}$ 1-Methyl-TIQ; (C) $100 \mu \mathrm{M} N$-Methyl-(R/S)Salsolinol; (D) $100 \mu \mathrm{M} N$-Methyl-Norsalsolinol; (E) $1 \mu \mathrm{M}$ Rotenone. (F) Normalized red:green fluorescence ratios of compounds after 15 min exposure. Values represent the mean +/SEM of three experiments with four wells in each treatment. Scale bar=25 $\mu \mathrm{m}$.

* Indicates significant difference between compounds and control $(P<0.05)$. 


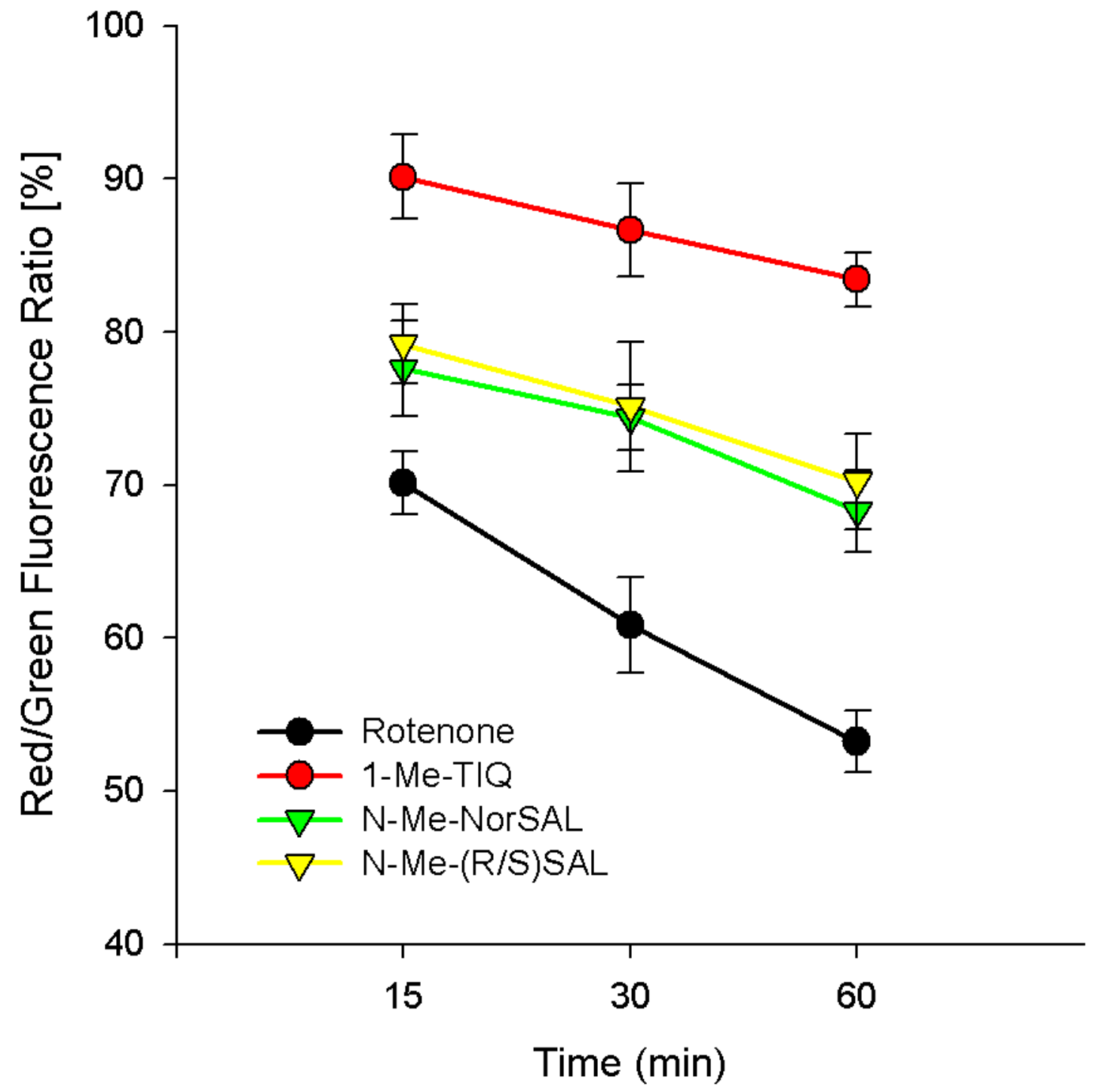

Figure 5-4. Red/Green fluorescence ratios of study compounds over 1 hour. Time-dependent decrease in $\Delta \Psi_{\mathrm{m}}$ in SH-SY5Y cells treated with $100 \mu \mathrm{M} 1$-methyl-TIQ, $N$-methyl- $(R / S)$ salsolinol or $N$-methyl-norsalsolinol. $1 \mu \mathrm{M}$ rotenone was utilized as positive control. Values represent the mean +/- SEM of three independent experiments. 


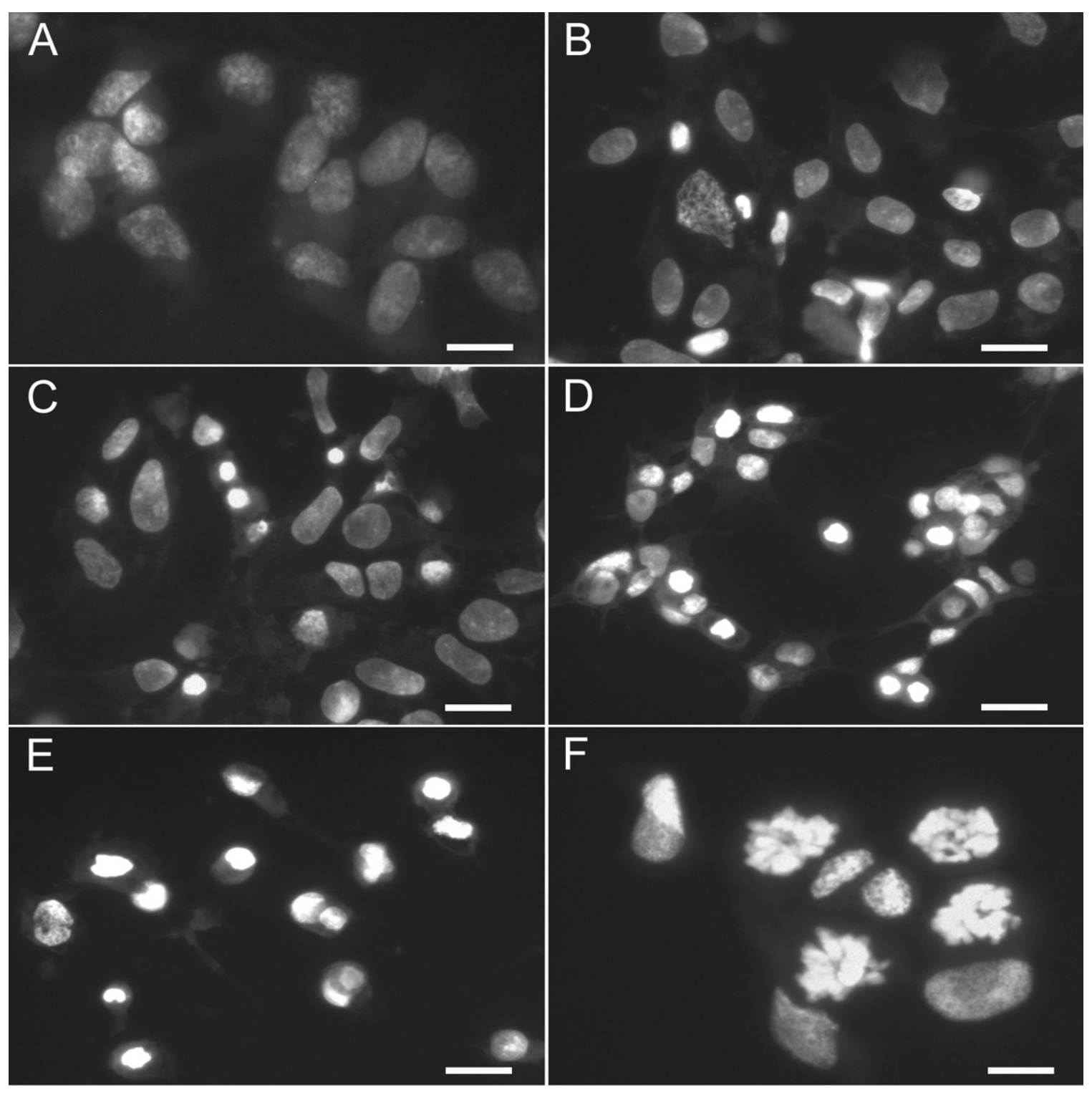

Figure 5-5. TIQ derivative-induced apoptosis of SH-SY5Y cells.

DAPI-stained nuclei are shown from (A) control cells and cells treated with (B) $100 \mu \mathrm{M} 1$ methyl-TIQ, (C) $100 \mu \mathrm{M} \mathrm{N}$-methyl-(R/S)salsolinol, (D) $100 \mu \mathrm{M} \mathrm{N}$-methyl-norsalsolinol and (E) $1 \mu \mathrm{M}$ rotenone for $1 \mathrm{hr}$. Small, pyknotic cells undergoing apoptosis are visible after exposure to all study compounds. Cells treated with $1 \mu \mathrm{M}$ rotenone $(\mathrm{F})$ depict the early and late stages of chromatin condensation and fragmentation characteristic of apoptosis. 
function of their polar oxygen molecules, rotenone, $N$-methyl-(R/S)salsolinol and $N$ methyl-norsalsolinol exhibited higher TPSA than 1-methyl-TIQ. TPSA is commonly used in medicinal chemistry for the optimisation of cell permeability. Molecules with a polar surface area of greater than $140 \AA^{2}$ are believed to be poor at permeating cell membranes. For penetration of the blood-brain-barrier, TPSA values should typically be less than $60 \AA^{2}$ (Ertl et al 2000). A significant positive correlation was observed between cytotoxicity ( $\mathrm{LC}_{50}$ value) and TPSA (correlation coefficient $r^{2}=0.92$ ), but not between cytotoxicity and log $P$ value $\left(r^{2}=0.06\right)$ or $V_{m}\left(r^{2}=0.34\right)$.

\subsection{DISCUSSION}

The present study demonstrates that $N$-methyl-(R/S)salsolinol, $N$-methylnorsalsolinol and 1-methyl-TIQ induce cell death in human SH-SY5Y dopaminergic neuroblastoma cells in a dose-dependent manner. Although the relative toxicity of $N$ methyl- $(R / S)$ salsolinol and $N$-methyl-norsalsolinol were very similar, $N$-methylnorsalsolinol was slightly more cytotoxic to SY5Y cells $\left(\mathrm{LC}_{50} 0.377 \mathrm{mM}\right.$ and $0.305 \mathrm{mM}$, respectively). 1-methyl-TIQ, however, was almost an order of magnitude less toxic to SY5Y cells ( $\left.L_{50} 2.153 \mathrm{mM}\right)$. Overall, rotenone $\left(\mathrm{LC}_{50} 0.013 \mathrm{mM}\right)$ was over 20 times more cytotoxic relative to either of the the $N$-methylated salsolinols. Cytotoxicity was positively correlated to TPSA, a measure of cell membrane permeability, for all study compounds. Studies of mitochondrial function indicate that these methylated TIQ derivatives induce an acute, time-dependent decrease in $\Delta \Psi_{\mathrm{m}}$, with potencies that closely mirror those of overall cellular viability. A collapse of $\Delta \Psi_{\mathrm{m}}$ is very likely accompanied by an impairment of overall cellular energy production without generation of reactive oxygen species. Finally, analysis of nuclear morphology indicates that cell death is likely apoptotic in nature.

$N$-methyl- $(R)$ salsolinol is arguably the best-studied of the TIQ derivatives in terms of cell death mechanisms. To date, several reports document the cytotoxicity of $\mathrm{N}$ methyl-(R)salsolinol in SH-SY5Y cells (Akao et al 2002; Akao et al 1999; Maruyama et al 2001; Maruyama et al 1997a; Maruyama et al 1997b; Maruyama et al 2004; Naoi et al 2000; Yi et al 2006a; Yi et al 2006b). Interestingly, at concentrations of $1 \mathrm{mM}$, the Senantiomer of $N$-methyl-salsolinol was determined to be significantly less toxic than the $R$-enantiomer in SY5Y cells using single-cell gel electrophoresis (the "comet assay") for detection of DNA fragmentation (Maruyama et al 1997a; Maruyama et al 1997b). Based on the results of these studies alone, the role of $N$-methyl-(S)salsolinol in dopaminergic cell death has been largely ignored. A later study by Akao and colleagues utilized 500 $\mu \mathrm{M} N$-methyl- $(R)$ salsolinol exposure to SY5Y cells for $72 \mathrm{hrs}$ to confirm apoptotic cell death using DNA ladder electrophoresis and detection of activated caspase 3 (Akao et al 1999). These results were later confirmed in a separate experiment demonstrating the loss of $\Delta \Psi_{\mathrm{m}}$, activation of caspase 3 and DNA fragmentation after $250 \mu \mathrm{M} \mathrm{N}$-methyl$(R)$ salsolinol exposure to SY5Y cells for $24 \mathrm{hrs}$ (Naoi et al 2000). Maruyama and colleagues demonstrated that $24 \mathrm{hr}$ exposure to $200 \mu \mathrm{M} \mathrm{N}$-methyl- $(R)$ salsolinol induced an acute decrease in $\Delta \Psi_{\mathrm{m}}$ (at $3 \mathrm{hrs}$ ) activation of caspases and fragmentation of DNA (Maruyama et al 2001). Akao and co-workers have also demonstrated that $500 \mu \mathrm{M} \mathrm{N}$ methyl- $(R)$ salsolinol reduced $\Delta \Psi_{\mathrm{m}}$ in the early phase of apoptosis and was inhibited by both Bcl-2 (a pan-caspase inhibitor) and cyclosporin A (an inhibitor or mitochondrial pore opening) independently, suggesting the dependence of the caspase cascade and the mitochondrial permeability transition in $N$-methyl- $(R)$ salsolinol-induced apoptosis (Akao 
et al 2002). This was also the first report to demonstrate that rasagiline ( $N$-propargyl1(R)-aminoindan), an irreversible MAO-B inhibitor, suppressed $\Delta \Psi_{\mathrm{m}}$ reduction, release of cytochrome $\mathrm{c}$ from mitochondria and apoptosis induced by $\mathrm{N}$-methyl- $(R)$ salsolinol in $\mathrm{SH}$ SY5Y cells (Akao et al 2002). The compound benzofuranylpropylaminopentane (BPAP; a catecholaminergic enhancer) was also shown to inhibit apoptosis by direct stabilization of $\Delta \Psi_{\mathrm{m}}$ and the induction of the anti-apoptotic protein Bcl-2 in SY5Y cells exoposed to $N$ methyl- $(R)$ salsolinol (Maruyama et al 2004). A recent study by Yi and colleagues demonstrated that the binding of $N$-methyl- $(R)$ salsolinol to mitochondria was inhibited by clorgyline, a MOA-A inhibitor (Yi et al 2006a). However, MAO-A inhibition does not apprear to be a prerequisite for the protective function of propargylamine derivatives, such as rasagiline, towards $N$-methyl- $(R)$ salsolinol-mediated toxicity (Yi et al $2006 \mathrm{~b}$ ). In aggregate, these studies suggest that $N$-methyl- $(R)$ salsolinol enters mitochondria via a MAO dependent mechanism and there induces apoptotic cell death by complex 1 inhibition with resultant destabilization of $\Delta \Psi_{\mathrm{m}}$, the release of cytochrome $\mathrm{c}$ from the mitochondrial matirx and induction of the caspase cascade.

Unfortunately, none of the above-mentioned reports utilized an array of $N$-methyl$(R)$ salsolinol dosages or an overall measure of cell viability, such as the MTT assay, to determine a standard convention of toxicity such as the $\mathrm{LC}_{50}$ or median lethal concentration. Without such a measure, a comparison between compounds or between reports becomes virtually impossible. However, our results, obtained using racemic $N$ methyl-(R/S)salsolinol are largely consistent with previous reports in respect to the acute collapse of $\Delta \Psi_{\mathrm{m}}$. It should be mentioned, however, that the results of Maruyama, Naoi and colleagues should be interpreted with caution. For instance, all of the abovemetioned reports utilize the synthetic methods of Teitel and colleagues for synthesis of racemic $N$-methyl-(R/S)salsolinol (Teitel et al 1972). However, no explanation or procedure for the difficult and expensive separation and purification of $R$ - and $S$ enantiomers is provided. Furthermore, to date, no publication has provided any physical data (such as NMR or mass spectra) to prove successful yield. With this in mind, the dominant toxicity of the $R$-enantiomer may be suspect.

$\mathrm{N}$-methyl-norsalsolinol, a compound known to inhibit MAO and $\mathrm{TH}$ in vitro, has received much less attention in terms of cytotoxity (Moser et al 1996a; Scholz et al 1997). The present study represents the first to quantify the definitive cytotoxicity of $\mathrm{N}$ methyl-norsalsolinol in dopaminergic neurons. Furthermore, our data suggests that the induced decrease in $\Delta \Psi_{\mathrm{m}}$ and overall toxicity of $N$-methyl-norsalsolinol is on par with that of $N$-methyl-(R/S)salsolinol in SY5Y cells. In agreement with our findings, $N$-methylnorsalsolinol was shown to be equally as toxic to cultured PC12 cells as N-methyl$(R)$ salsolinol (Maruyama et al 1993; Naoi et al 1995). However, it should mentioned that that PC12 cells are well known to rely far more upon glycolysis than the tricarboxylic acid cycle for their energy supply, and may thus be less sensitive to complex 1 inhibitormediated cytotoxicity (Basma et al 1992). Nevertheless, both $N$-methyl-(R/S)salsolinol and $\mathrm{N}$-methyl-norsalsolinol appear to be at least twice as cytotoxic as their parent (R/S)salsolinol in SY5Y cells ( LC $_{50} 0.704 \mathrm{mM}$ ) (Storch et al 2000). This finding suggests that methylation at the $N$-position does confer heightened toxicity to salsolinol derivatives.

The observed cytotoxicity of 1-methyl-TIQ in SH-SY5Y cells $\left(\mathrm{LC}_{50} 2.153 \mathrm{mM}\right)$ is consistent with a recent study by Kotake and colleagues ( $\mathrm{LC}_{50} 2.81 \mathrm{mM}$ ) (Kotake et al 2007). In this report, the complex I inhibiton and resultant cytotoxicity of several TIQ 
derivatives were determined in SH-SY5Y cells. Overall, N-methyl-TIQ and 1-methyl-TIQ were the least cytotoxic of all direct TIQ derivatives under study $\left(\mathrm{LC}_{50} 2.63 \mathrm{mM}\right.$ and 2.81 $\mathrm{mM}$, respectively). For reference, the reported $\mathrm{LC}_{50}$ of TIQ in SY5Y cells was $1.19 \mathrm{mM}$. These results may explain the finding that the inhibitory effect on mitochondrial respiration induced by MPP+ in isolated rat liver mitochondria is prevented by the addition of 1-methyl-TIQ (Parrado et al 2000). As suggested by the authors, 1-methylTIQ may bind to complex 1 of mitochondria and effectively shield the enzyme system from compounds with orders of magnitude higher toxicity such as MPP+ $\left(\mathrm{LC}_{50}\right.$ approaching $0.001 \mathrm{mM}$ in SY5Y cells). However, based on its relatively weak toxicity, it may still induce a decrease in $\Delta \Psi_{\mathrm{m}}$ and initiate cell death on much longer time scales. Thus, 1-methyl-TIQ does not necessarily appear to be neuroprotective, but rather less deadly.

Another mechanism which may contribute to the strong effects of $N$-methylnorsalsolinol and $N$-methyl-(R/S)salsolinol on mitochondrial indices of cellular energy might be their mild inhibitory effect on the $\alpha$-ketoglutarate dehydrogenase complex ( $\alpha$ $\mathrm{KGDH}$ ) of the TCA cycle (McNaught et al 1995a). Since $\alpha-\mathrm{KGDH}$ catalyses the oxidation of $\alpha$-ketoglutarate to succinate, which is a substrate for mitochondrial complex II, the blockade of $\alpha-K G D H$ would additionally inhibit electron transfer via complex II. Since the metabolism of $\alpha$-ketoglutarate by $\alpha-K G D H$ produces NADH, reduction of $\alpha$ KGDH activity would partially inhibit complex I by decreasing NADH and, in addition, inhibition of the rate-limiting enzyme $\alpha-\mathrm{KGDH}$ would lead to impairment of the general function of the TCA cycle. Interestingly, a decrease in $\alpha-K G D H$ activity has been demonstrated in the SN of PD patients (Mizuno et al 1994).

It should also be mentioned that the presented data do not rule out the possibility that free radical formation is involved in the impairment of cellular energy production induced by $N$-methyl-norsalsolinol and $N$-methyl-(R/S)salsolinol. Production of hydroxyl radicals through oxidation of $N$-methylated salsolinols has been reported under in vitro conditions (Naoi et al 1998a; Nappi et al 1999). On the other hand, $N$-methyl$(R)$ salsolinol has been shown to scavenge hydroxyl radicals produced by oxidation of dopamine in vitro and in vivo (Naoi et al 1998a). However, by using the same cell line and similar experimental conditions, Willets and colleagues demonstrated that antioxidative substances, such as ascorbic acid and atocopherol, failed to protect $\mathrm{SH}$ SY5Y cells against $(R / S)$ salsolinol-induced toxicity, suggesting that free radical formation does not play a major role in salsolinol derivative-induced toxicity in this cell system (Willets et al 1995).

The results presented herein also demonstrate a significant positive correlation between cytotoxicity and TPSA for all compounds under study. TPSA, defined simply as the molecular surface corresponding to oxygen and nitrogen, is one of the most important parameters used to characterize the transport properties of molecules. TPSA values have shown very good correlations with intestinal absorption, cell membrane solubility and blood-brain barrier penetration (Clark 1999a;b). Based on TPSA calculations, all study compounds have a polar area less than $140 \AA^{2}$, making all compounds good candidates for permeating cell membranes. This observation may suggest that TIQ derivatives may induce cellular apoptosis independent of active membrane transport. However, TIQ derivatives with very high TPSA and very low log P values may be too hydrophilic for passive membrane transport and may require transporters such as the DAT for toxicity. 


\subsection{CONCLUSIONS}

The endogenous neurotoxins $N$-methyl-norsalsolinol and $N$-methyl$(R / S)$ salsolinol, which are structurally similar to MPTP, have been reported to inhibit mitochondrial complex I (NADH-Q reductase) activity as does the MPTP metabolite $M P P+$. However, the mechanism of $N$-methyl-norsalsolinol and $N$-methyl- $(R / S)$ salsolinol leading to neuronal cell death is largely unknown. Our results demonstrate that these $N$ methylated salsolinols are toxic to dopaminergic neuroblastoma SH-SY5Y cells by impairment of mitochondrial function. This impairment is most likely caused by inhibition of mitochondrial complex I activity, and possibly enhanced by blocking the general function of the TCA cycle. $N$-methyl-norsalsolinol and $N$-methyl-(R/S)salsolinol induce a dose-dependent decrease in cell survival with $\mathrm{LC}_{50}$ values of $0.305 \mathrm{mM}$ and $0.377 \mathrm{mM}$ after $24 \mathrm{hrs}$, respectively. Another methylated TIQ derivative with suspected neuroprotective properties, 1-methyl-TIQ, was shown to be an order of magnitude less toxic to SY5Y cells ( $\left.\mathrm{LC}_{50} 2.153 \mathrm{mM}\right)$. Rotenone, a potent inhibitor of mitochondrial complex I, was over 20 times more cytotoxic relative to either of the the $\mathrm{N}$-methylated salsolinols $\left(\mathrm{LC}_{50} 0.013 \mathrm{mM}\right)$. Using the $\mathrm{JC}-1$ dye, it was determined that these methylated TIQ derivatives induce an acute, time-dependent collapse of $\Delta \Psi_{\mathrm{m}}$, likely accompanied by an impairment of overall cellular energy production. Analysis of nuclear morphology confirmed that cell death is likely apoptotic in nature. This data confirms that $\mathrm{N}$-methylated salolinol derivatives are indeed toxic to human dopaminergic neurons. Their synthesis within dopaminergic regions of the brain coupled with the potential for accumulation from exogenous sources make them strong candidates for a neurotoxicological component to the pathobiology of PD. 


\section{CHAPTER 6. SUMMARY AND DISCUSSION}

\subsection{SUMMARY}

The research presented herein represents a comprehensive and systematic evaluation of TIQ accumulation in the brain via endogenous and/or exogenous sources and their relationship to neurodegenerative disease. First, using liquid chromatographic and immunocytochemical techniques the regional distribution of several TIQs were determined in mouse, rat, normal human and PD human brain. The cellular localization of an important and relatively unknown TIQ, N-methyl-NorSAL, was determined in rodent brain using a novel monoclonal antibody. Second, the content of several TIQs were determined in 40 commonly consumed plants using liquid chromatography. The intake of these foods and their toxin profiles were then explored in relation to PD using a lifetime food-serving intake questionnaire. Using rat brain samples from several age groups, the accumulation of TIQs over time was demonstrated in several brain regions. Finally, using a model for human dopaminergic neurons, the dose-dependent cytotoxicity of select $N$-methylated TIQs was demonstrated and associated with mitochondrial dysfunction. Presented as a supplement to the primary studies, the thermal stability and molecular physical properties of TIQs were determined.

Several members of the TIQ family of monoamine alkaloids can be formed from dopamine or its oxidized metabolites and may be involved in the pathogenesis of monoaminergic cell death in PD. Many salsolinol derivatives were detected at higher concentrations in regions of increased dopamine synthesis and turnover such as the ventral midbrain and striatum, respectively. Furthermore, salsolinols were present at higher concentrations than TIQ and its benzyl and methyl derivatives, especially in human brain. Both enantiomers of SAL were found in all brain regions, with noticeable correlations identified between stereoselective synthesis of $(R) S A L$ and dopamine concentration. A possible consequence of nigrostriatal dopaminergic cell death, significantly lower levels of $(R) S A L,(S) S A L, N$-methyl-(R)SAL and $N$-methyl-(S)SAL were detected in the caudate nuclei of PD in comparison with normal human brain. Overall, the methylated and benzylated derivatives of TIQ were present at lower concentrations than their parent compound. In the brain, topological trends in derivative concentration suggest regionally distinct methylation patterns. Finally, stability experiments revealed high thermal tolerance for all TIQs.

The TIQ derivative $N$-methyl-NorSAL is present in normal human brain, has been identified in the CSF of PD patients and can be derived from dopamine by condensation with aldehydes or $\alpha$-ketoacids. Using HPLC-EC, $N$-methyl-NorSAL was detected in all regions of rodent and human brain. In rodent brain, $N$-methyl-NorSAL concentrations were similar among frontal cortex, ventral midbrain, striatum, hippocampus and cerebellum. Conversely, in normal human brain, $N$-methyl-NorSAL was concentrated in dopaminergic regions (SN and striatum). N-methyl-NorSAL levels were significantly lower in the SN and caudate nuclei from PD patients, a finding possibly related to the

death of nigrostriatal neurons. Furthermore, $N$-methyl-NorSAL-IR co-localized with a general neuronal marker and a monoaminergic marker. The widespread neuronal localization of $\mathrm{N}$-methyl-NorSAL in several mammalian species suggests that this compound acts as a relatively weak neurotoxin. 
Epidemiological studies suggest that environmental factors contribute to the pathogenesis of PD. Several TIQs have been shown to inhibit complex I of the ETC, initiate apoptosis in cultured dopaminergic neurons and are recognized as latent substances in plants. High concentrations of TIQ derivatives were detected in banana, cherry, peach, grapefruit, avocado, button mushroom, leaf lettuce and celery. Enantiomeric salsolinol derivatives were detected as racemic mixtures, indicating that stereoselective enzymatic synthesis does not occur in plants. The mushroom family (Agaricaceae) contained the highest average TIQ derivative load, salsolinol derivative load and total toxin load of all plant families analyzed. The banana family (Musaceae), rose family (Rosaceae), sunflower family (Asteraceae) and pineapple family (Bromeliaceae) also contained particularly high average neurotoxin loads. Utilizing a lifetime food-item intake questionnaire, a trend of increased intake of several plants products (bananas, apples, watermelon, oranges, cantaloupe, grapes, grapefruit, potatoes, iceberg lettuce, tomatoes, onions, carrots and celery) was observed in PD patients. However, this finding only reached significance in oranges. Members of the rue (Rutaceae), banana (Musaceae), gourd (Cucurbitaceae), carrot (Apiaceae) and nightshade (Solanaceae) families were found to be highly consumed by both normal and PD humans, and to concentrate several potentially neurotoxic TIQ derivatives.

Using HPLC-EC and LC-MS/MS, the accumulation of TIQ derivatives in rat brain was demonstrated over time. The concentration of TIQ derivatives appears to change very little over time, with high accumulation within the first several weeks of life. Salsolinol derivatives experience a more rapid ascent within the first 6 months of life within dopaminergic regions, followed by another rise in concentration in late life. Levels of $N$-methyl-NorSAL accumulate more slowly, followed by rapid accumulation around 6 months with sustained increases over time. Overall, the net content of TIQ and salsolinol derivatives rises early and remains relatively constant over the life of the rat, with the exception of $\mathrm{N}$-methyl-NorSAL.

Finally, we demonstrate that the endogenous TIQs $N$-methyl-NorSAL and $N$ methyl- $(R / S) S A L$ are toxic to dopaminergic neuroblastoma SH-SY5Y cells by impairment of mitochondrial function. $N$-methyl-NorSAL and $N$-methyl- $(R / S) S A L$ induce a dosedependent decrease in cell survival with $L_{50}$ values of $0.305 \mathrm{mM}$ and $0.377 \mathrm{mM}$ after $24 \mathrm{hrs}$, respectively. 1-methyl-TIQ, was shown to be an order of magnitude less toxic to SY5Y cells ( $\left.\mathrm{LC}_{50} 2.153 \mathrm{mM}\right)$, potentially explaining its suggested relative neuroprotective properties. Rotenone, a potent inhibitor of mitochondrial complex I, was over 20 times more cytotoxic relative to either of the $N$-methylated salsolinols $\left(\mathrm{LC}_{50} 0.013 \mathrm{mM}\right)$. A significant positive correlation between cytotoxicity and polar surface area, a measure of membrane permeability, was also observed for these compounds. All of the methylated TIQ derivatives examined induce an acute, time-dependent collapse of $\Delta \Psi_{\mathrm{m}}$, suggesting an impairment of overall cellular energy production. Analysis of nuclear morphology confirmed that cell death is apoptotic in nature.

Several important issues regarding the toxicity of TIQs in the brain are specifically addressed by our results. First, our studies of TIQ detection in animal and plant tissues are of the highest standard and are unparalleled in comparison to the published literature. Our experiments represent a fundamental and thorough analysis in a controversial and poorly understood realm of neurobiology. We demonstrate that TIQs are found within neurons of many brain regions in both rodents and humans, suggesting that glial cells may not be necessary for toxicity and that these toxins may cause cell death throughout the brain. We demonstrate that $(R / S) S A L$ and its derivatives are found 
at higher levels in dopaminergic regions of human brain and that these compounds increase as the content of dopamine increases in the rat brain, both of which support the hypothesis that salsolinol derivatives may be synthesized from dopamine within neurons (Cohen \& Collins 1970; Schöpf \& Bayerle 1934). Second, our quantative data in plants is the first to truly establish that TIQs are indeed natural substances found in the environment at sufficient concentrations to allow for accumulation in the brain after intake. Furthermore, data obtained from our food-intake questionnaire suggests that PD patients may ingest higher quantities of select fruits and vegetables that contain relatively high levels of several TIQ derivatives. These results provide evidence to support a pragmatic exogenous means of neurotoxin exposure. Examination of the physical properties presented in Appendix A, in conjunction with previous data concerning the blood-brain barrier permeability of select TIQ derivatives (Abe et al 2001; Origitano et al 1981; Song et al 2006; Thumen et al 2002) suggests that most TIQs are good candidates for penetration of the gastrointestinal mucosa after oral ingestion and subsequent penetration into the brain. These findings definitively place potentially cytotoxic TIQ derivatives within dopaminergic regions of the brain essential to the pathophyisology of PD. Finally, our experiments demonstrating the in vitro toxicity of $\mathrm{N}$ methylated salsolinols in dopaminergic neurons definitively confirms the findings of other groups (Akao et al 2002; Akao et al 1999; Maruyama et al 2001; Maruyama et al 1997a; Maruyama et al 1997b; Maruyama et al 2004; Naoi et al 2000; Yi et al 2006a; Yi et al $2006 \mathrm{~b}$ ) and establishes a relative measure of their toxicity. They also demonstrate, for the first time, that the toxicity of $\mathrm{N}$-methyl-NorSAL is higher than that of $\mathrm{N}$-methyl$(R / S) S A L$ in dopaminergic neurons. In conclusion, these results provide strong support for our global hypothesis of TIQ accumulation and concentration in dopaminergic regions of human brain over time via both endogenous and exogenous means, leading to gradual mitochondrial dysfunction and ultimate neuronal cell death with subsequent development of parkinsonian symptomatology.

\subsection{DISCUSSION}

The potential role of TIQs in the pathobiology of PD places the mitochondrion in a centralized role in initiating cell death and provides an avenue for the interaction of several genes known to be associated with the development of disease. As such, the function of the mitochondrion in apoptosis and the role of neurotoxins (such as the TIQs) superimposed on genetic susceptibility warrants further discussion.

Over the past three decades, two fundamentally different forms of cell death, apoptosis and necrosis, have been defined. Necrosis is a passive process that occurs when a major environmental damaging event causes irreversible cellular dysfunction. This results in cell swelling with cell membrane breakdown and release of cellular contents and is usually associated with an inflammatory response. Apoptosis, on the other hand, is a well defined active process that involves shrinkage of the cell, breakdown of cellular proteins, condensation of the nucleus, and cleavage of nuclear DNA, and typically does not cause inflammation (Cummings et al 1997; Honig \& Rosenberg 2000). Apoptosis research has undergone a change from a paradigm in which the nucleus determines the apoptotic process to one in which mitochondria are a major center of death control (Aizenman et al 2000; Brenner \& Kroemer 2000; Joza et al 2001). It is now clear that many different apoptotic signals converge on mitochondria. A variety of key events in apoptosis focus on mitochondria, including changes in electron transport, loss of mitochondrial transmembrane potential, altered cellular oxidation- 
reduction potential, release of caspase regulators, and participation of pro- and antiapoptotic Bcl-2 family proteins (Aizenman et al 2000; Bernardi et al 1999; Brenner \& Kroemer 2000; Kroemer 1999).

In the three-step model of apoptosis proposed by Kroemer and colleagues,three phases can be distinguished during apoptosis, an initiation phase, which is extremely heterogeneous, during which signal transduction cascades or damage pathways are activated, a decision phase, during which the cell decides to die, and a degeneration phase, during which proteins released from mitochondria cause the activation of programmed cell death through the activation of caspases and nucleases (Brenner \& Kroemer 2000; Kroemer 1999; Marzo et al 1998). During the decision phase mitochondria integrate different death signals and trigger the decision to die by releasing proapoptotic proteins. The mitochondrial intermembrane space contains a number of cell death-promoting factors, including cytochrome $c$, second mitochondria derived activator of caspase/direct IAP binding protein with low pl (Smac/DIABLO), apoptosisinducing factor (AIF) and procaspases (Aizenman et al 2000; Chai et al 2000; Joza et al 2001). The released cytochrome $c$ interacts with procaspase 9 , apoptotic peptidase activating factor 1 (Apaf-1), and ATP to trigger the assembly of the apoptosome which proteolytically activates caspase- 9 and other "executioner phase" caspases (Skulachev 1998; Yuan \& Yankner 2000). When the caspases are activated they cleave a wide variety of proteins in the cell, which results in cell death via apoptosis. The caspase9/caspase-3 pathway is also regulated by proteins of the IAP (inhibitor of apoptosis proteins) family. One of these, X-linked inhibitor of apoptosis protein (XIAP), binds to caspase- 9 and inhibits its proteolytic activity. The caspase-inhibiting effects of XIAP are antagonized by another intermembrane protein, Smac/DIABLO (Chai et al 2000; Meier et al 2000). AIF is a flavoprotein normally confined to the mitochondrial intermembrane space. Once released to the cytosol, AIF translocates to the nucleus and induces nuclear chromatin condensation, as well as large scale DNA fragmentation in a caspaseindependent fashion (Joza et al 2001; Marzo et al 1998).

Direct evidence of mitochondrial dysfunction leading to disease is gleaned from clinical manifestations caused by mitochondrial DNA (mtDNA) mutations. Mitochondrial dysfunction due to defects in oxidative phosphorylation, such as those induced by complex I inhibitors, increases reactive oxygen species production and leads to accumulated mitochondrial oxidative damage, energy insufficiency, cell dysfunction and progressive physiological dysfunction in tissues requiring a large supply of energy (Esposito et al 1999; James et al 1996). Several particular mtDNA polymorphisms and haplotypes have been reported to be associated with the risk of PD, and mutations in mtDNA or in the nuclear-encoded mtDNA polymerase-G (POLG) cause PD-like symptoms (Autere et al 2004; Luoma et al 2007). Recently, the finding that the electron acceptor coenzyme $Q_{10}$, also a potent antioxidant, can slow the progressive deterioration of function in PD provides a further clue that decreasing mitochondrial oxidative stress may alter the progression of this disease. Therefore, mitochondrial dysfunction, resulting from genetic defects, endogenous/environmental toxins, or a combination of the two, may cause oxidative modification of $\alpha$-synuclein that leads to selective neurodegeneration by means of oxidative stress (Sherer et al 2003).

Only a small fraction of PD cases are caused by single-gene mutations. However, the identification of these rare, inherited mutations causing familial forms of PD have provided much insight into the discovery of novel proteins and pathways that are likely to be relevant in the pathogenesis of both the genetic and sporadic forms of 
PD. These genes include $\alpha$-synuclein, parkin, PINK1, DJ-1 and LRRK2. The demonstration that $\alpha$-synuclein is the main constituent of LBs leads to the suggestion that cell degeneration may arise through the $\alpha$-synuclein-related pathway, protein misfolding and aggregation. These proteins are ubiquitinated and initially degraded by the UPS, in which parkin acting as an E3 ligase in the UPS system has a crucial role. The identification of mutations in DJ-1 as a possible redox sensor, and phosphatase and tensin homologue PINK1, a mitochondrial kinase, provided further evidence that mitochondrial dysfunction and oxidative stress might have a primary role in the pathogenesis of PD (Bonifati et al 2003; Valente et al 2004). PINK1 encodes a putative serine/threonine kinase with a mitochondrial targeting sequence. Studies have revealed that PINK1 is associated with the inner mitochondrial membrane and is exposed to the intermembrane space (Gandhi et al 2006; Silvestri et al 2005). Parkin localizes predominantly to the cytosol but also associates with the mitochondrial outer membrane. PINK1 might modulate the activity or stability of parkin either within the mitochondrion or in the cytosol, as it might be released from mitochondria under certain conditions (Dodson \& Guo 2007). Moreover, in Drosophila, it has been shown that PINK1 and parkin function, at least in part, in the same pathway. These studies suggest a role for PINK1 in normal mitochondrial function and imply that parkin is downstream of PINK1 (Clark et al 2006; Gautier et al 2008). The findings that the PINK1/parkin pathway promotes mitochondrial fission and/or inhibits fusion in Drosophila and that the loss of mitochondrial integrity in PINK1 and parkin mutants derives from reduced mitochondrial fission further support the possibility that PINK1 and parkin might regulate mitochondrial dynamics (Deng et al 2008; Poole et al 2008). Obviously, neurotoxins causing dysfunction of existing mitochondria in the setting of these mutations would be disastrous for the cell.

Mitochondrial functions are performed in concert with other cell compartments and are regulated by various extracellular and intracellular signals. A host of nuclear receptors and other nuclear transcription factors, such as nuclear factor kappa-lightchain-enhancer of activated $B$ cells (NF- $\kappa B$ ), activator protein-I (AP-1), cAMP response element binding protein (CREB) and protein 53 (p53), involved in growth, metabolic and developmental processes, have been detected in mitochondria (Psarra \& Sekeris 2008). Recently, the idea that these mitochondrially localized transcription factors can act not only on the nucleus but also on mitochondrial transcription has gained support. To adapt to a changing environment, it is necessary for cells to be able to regulate transcription of genes serving a common function by way of interaction with common binding sites in the two genomes including nuclei and mitochondria. Oxidative stress and the redox state are also involved in the survival signaling pathway of stressed cells (Reddehase et al 2009). In response to increased oxidative stress, there may be some alteration in dopaminergic neurons in terms of mitochondrial abundance, copy number and integrity of mtDNA under these pathological conditions. Within its tolerable threshold, reactive oxygen species, induced by neurotoxin exposure, may activate protective responses through expression of specific genes to aid cells in coping with hazardous environments. Once beyond this threshold, these reactive oxygen species may cause damage to mtDNA and other biomolecules of the affected dopaminergic neurons and elicit an apoptotic cascade induction of mitochondrial membrane permeability transition and release of proapoptotic proteins such as cytochrome $c$, which eventually leads to dopaminergic neuron degeneration. Being the major supplier of energy in mammalian cells, mitochondria are necessary to provide more energy for damage repair and cellular survival during disease processes with excessive oxidative 
stress. In order to meet the demand for energy supply, signals transmitted to the nucleus may induce mitochondrial proliferation and mtDNA amplification to produce more functional mitochondria. The abundance of mitochondria in a cell is determined by the biogenesis and division of the organelles and tightly controlled by the activation of specific transcription factors encoded by nuclear genes (Attardi \& Schatz 1988). Nuclear respiratory factors 1 and 2 (NRF-1 and NRF-2) are transcriptional regulators that act on the nuclear genes coding for peptides necessary for the mitochondrial ETC. They also regulate the expression of many other genes involved in mtDNA replication (Evans \& Scarpulla 1990; Virbasius et al 1993). Mitochondrial transcription factor A (Tfam) acts on promoters within mtDNA and regulates the replication and transcription of the mitochondrial genome (Virbasius \& Scarpulla 1994). Both NRF-1 and NRF-2 can regulate the expression of the Tfam gene by binding to consensus-binding sites. This provides a unique mechanism for the cell to integrate the expression of nuclear DNAencoded proteins with the transcription of genes encoded by mtDNA. Recently, evidence has emerged that peroxisome proliferator-activated receptor gamma coactivator-1 $\alpha$ (PGC-1 $\alpha$ ) is a major regulator of mitochondrial biogenesis (Puigserver et al 2003). PGC-1 $\alpha$ regulates the expression of transcription factors involved in the coordinated expression of mitochondrial genes, such as NRF-1 and NRF-2, which in turn trigger the expression of nuclear genes coding for polypeptides of the ETC and proteins involved in transcription and replication of mtDNA (Puigserver et al 2003). Recently, the production of a Tfam knockout mouse model of PD, and the reduced mtDNA expression and ETC deficiency in the midbrain dopaminergic neurons of these animals further support this hypothesis (Ekstrand et al 2007). It thus becomes evident that in neurons stressed by mitochondrial toxins, a thorough understanding of pro-survival pathways of nigrostriatal neurons is crucial for the prevention of disease progression.

\subsection{FUTURE DIRECTIONS}

In conclusion, the body of work included in this dissertation provides important new insights into the role of TIQ derivatives in PD and points out clear avenues for future studies. One apparent starting point would be confirmation of TIQ-induced apoptosis in $5 Y$ cells using western blot analysis for detection of a subset of the above-mentioned mitochondrial proteins. Although apoptosis and necrosis may not be mutually exclusive in the setting of TIQ-mediated cell death, mechanistically related toxins such as rotenone tend to exclusively initiate apoptosis in dopaminergic cells.

Subsequent studies would involve a more precise determination of mechanisms involved in TIQ accumulation. By obtaining a more expansive collection of normal human brain samples from all decades of life, a TIQ derivative accumulation profile could be obtained and compared to that of the rodent. This data would be central to our hypothesis of time-dependent accumulation of TIQs in the human brain.

A logical experimental progression would then lead towards exploring our hypothesis of cytosolic dopamine and its relation to TIQ derivative production/accumulation. This would involve the utilization of mouse strains harboring mutations in proteins which contribute to the regulation of vesicular and cytosolic dopamine concentrations. VMAT2 is a transmembrane protein responsible for uptake of cytosolic monoamines within neurons. Based upon our hypothesis, VMAT2 may play a role in neuroprotection by sequestering dopamine into vesicles and away from TIQ derivative biosynthesis. Previous neurochemical analysis of VMAT2-deficient mice 
shows general age-dependent reductions in brain tissue levels of dopamine, norepinephrine, epinephrine and serotonin by approximately $80-90 \%$, which is accompanied by increased dopamine and serotonin turnover in multiple regions of the brain (Caudle et al 2007; Colebrooke et al 2006; Mooslehner et al 2001). Furthermore, loss of VMAT2 function causes gradual neurodegeneration in the SNpc and locus ceruleus of aged VMAT2-deficient mice. To date, TIQ derivative concentrations have not been measured in these mice. A very interesting experiment would involve chromatographic analysis of multiple brain regions of these animals over various ages.

Another presynaptic protein involved in the pathophysiology of PD is $\alpha$-synuclein, whose overexpression causes PD in humans. Interestingly, $\alpha$-synuclein knockdown has been shown to increase the density of VMAT2 molecules, and VMAT2 has been shown to co-immunoprecipitate with $\alpha$-synuclein (Fountaine et al 2008). Mice overexpressing wild-type human $\alpha$-synuclein have excellent construct validity as models of sporadic PD. These mice reproduce pathological findings in early PD, demonstrate regionally selective accumulation of insoluble $\alpha$-synuclein and exhibit multiple deficits observed in early PD, including olfactory deficits and autonomic dysfunction (Fleming et al 2006). Therefore, they may represent very early stages of the disease and provide information on mechanisms that initiate the pathophysiological cascades in PD. Chromatographic analysis of TIQ derivatives in the brain of these mice at various time points would provide insight into the potentially toxic consequences of $\alpha$-synuclein overexpression. Comparison of these values with those of VMAT2-deficient mice may also shed light on the interaction of these proteins within neurons. Based upon our hypotheses, $\alpha-$ synuclein overexpression would reduce VMAT2 levels and lead to increased production of TIQ derivatives and eventually cell death.

Ultimately, analysis of TIQ derivatives in these mutant strains could provide valuable information on accumulation in human brain and allow for the formulation of new hypotheses concerning intracellular trafficking and production/storage or interaction with additional intracellular systems. 


\section{LIST OF REFERENCES}

Aasly JO, Toft M, Fernandez-Mata I, Kachergus J, Hulihan M, et al. 2005. Clinical features of LRRK2-associated Parkinson's disease in central Norway. Ann Neurol $57: 762-5$

Abe K, Taguchi K, Wasai T, Ren J, Utsunomiya I, et al. 2001. Biochemical and pathological study of endogenous 1-benzyl-1,2,3,4-tetrahydroisoquinoline-induced parkinsonism in the mouse. Brain Res 907:134-8

Abeliovich A, Schmitz Y, Farinas I, Choi-Lundberg D, Ho WH, et al. 2000. Mice lacking alpha-synuclein display functional deficits in the nigrostriatal dopamine system. Neuron 25:239-52

Aharon-Peretz J, Rosenbaum H, Gershoni-Baruch R. 2004. Mutations in the glucocerebrosidase gene and Parkinson's disease in Ashkenazi Jews. N Engl J Med 351:1972-7

Aizenman E, Stout AK, Hartnett KA, Dineley KE, McLaughlin B, Reynolds IJ. 2000. Induction of neuronal apoptosis by thiol oxidation: putative role of intracellular zinc release. J Neurochem 75:1878-88

Akao Y, Maruyama W, Shimizu S, Yi H, Nakagawa Y, et al. 2002. Mitochondrial permeability transition mediates apoptosis induced by $\mathrm{N}$-methyl(R)salsolinol, an endogenous neurotoxin, and is inhibited by $\mathrm{Bcl}-2$ and rasagiline, $\mathrm{N}$-propargyl-1(R)aminoindan. J Neurochem 82:913-23

Akao Y, Nakagawa Y, Maruyama W, Takahashi T, Naoi M. 1999. Apoptosis induced by an endogenous neurotoxin, $\mathrm{N}$-methyl(R)salsolinol, is mediated by activation of caspase 3. Neurosci Lett 267:153-6

Alam ZI, Daniel SE, Lees AJ, Marsden DC, Jenner P, Halliwell B. 1997a. A generalised increase in protein carbonyls in the brain in Parkinson's but not incidental Lewy body disease. J Neurochem 69:1326-9

Alam ZI, Jenner A, Daniel SE, Lees AJ, Cairns N, et al. 1997b. Oxidative DNA damage in the parkinsonian brain: an apparent selective increase in 8-hydroxyguanine levels in substantia nigra. J Neurochem 69:1196-203

Albert ML, Feldman RG, Willis AL. 1974. The 'subcortical dementia' of progressive supranuclear palsy. J Neurol Neurosurg Psychiatry 37:121-30

Ancolio K, Alves da Costa C, Ueda K, Checler F. 2000. Alpha-synuclein and the Parkinson's disease-related mutant Ala53Thr-alpha-synuclein do not undergo proteasomal degradation in HEK293 and neuronal cells. Neurosci Lett 285:79-82

Anderson C, Checkoway H, Franklin GM, Beresford S, Smith-Weller T, Swanson PD. 1999. Dietary factors in Parkinson's disease: the role of food groups and specific foods. Mov Disord 14:21-7 
Antkiewicz-Michaluk L, Krygowska-Wajs A, Szczudlik A, Romanska I, Vetulani J. 1997. Increase in salsolinol level in the cerebrospinal fluid of parkinsonian patients is related to dementia: advantage of a new high-performance liquid chromatography methodology. Biol Psychiatry 42:514-8

Antkiewicz-Michaluk L, Michaluk J, Mokrosz M, Romanska I, Lorenc-Koci E, et al. 2001. Different action on dopamine catabolic pathways of two endogenous 1,2,3,4tetrahydroisoquinolines with similar antidopaminergic properties. J Neurochem 78:100-8

Aoyama K, Matsubara K, Kondo M, Murakawa Y, Suno M, et al. 2001. Nicotinamide-Nmethyltransferase is higher in the lumbar cerebrospinal fluid of patients with Parkinson's disease. Neurosci Lett 298:78-80

Askenasy JJ. 1981. Sleep patterns in extrapyramidal disorders. Int J Neurol 15:62-76

Attardi G, Schatz G. 1988. Biogenesis of mitochondria. Annu Rev Cell Biol 4:289-333

Autere J, Moilanen JS, Finnila S, Soininen H, Mannermaa A, et al. 2004. Mitochondrial DNA polymorphisms as risk factors for Parkinson's disease and Parkinson's disease dementia. Hum Genet 115:29-35

Autere JM, Moilanen JS, Myllyla VV, Majamaa K. 2000. Familial aggregation of Parkinson's disease in a Finnish population. J Neurol Neurosurg Psychiatry 69:107-9

Axelrod J, Kopin IJ, Mann JD. 1959. 3-Methoxy-4-hydroxyphenylglycol sulfate, a new metabolite of epinephrine and norepinephrine. Biochim Biophys Acta 36:576-7

Baba M, Nakajo S, Tu PH, Tomita T, Nakaya K, et al. 1998. Aggregation of alphasynuclein in Lewy bodies of sporadic Parkinson's disease and dementia with Lewy bodies. Am J Pathol 152:879-84

Bail M, Miller S, Cohen G. 1980. Selective 7-0-methylation of salsolinol in rat brain and heart in vivo. Life Sci 26:2051-60

Ballard PA, Tetrud JW, Langston JW. 1985. Permanent human parkinsonism due to 1methyl-4-phenyl-1,2,3,6-tetrahydropyridine (MPTP): seven cases. Neurology 35:949-56

Barbeau A, Roy M, Bernier G, Campanella G, Paris S. 1987. Ecogenetics of Parkinson's disease: prevalence and environmental aspects in rural areas. Can J Neurol Sci 14:3641

Basma AN, Heikkila RE, Saporito MS, Philbert M, Geller HM, Nicklas WJ. 1992. 1Methyl-4-(2'-ethylphenyl)-1,2,3,6-tetrahydropyridine-induced toxicity in PC12 cells is enhanced by preventing glycolysis. J Neurochem 58:1052-9

Bembenek ME, Abell CW, Chrisey LA, Rozwadowska MD, Gessner W, Brossi A. 1990. Inhibition of monoamine oxidases $A$ and $B$ by simple isoquinoline alkaloids: racemic and optically active 1,2,3,4-tetrahydro-, 3,4-dihydro-, and fully aromatic isoquinolines. $J$ Med Chem 33:147-52 
Benabid AL, Pollak P, Gao D, Hoffmann D, Limousin P, et al. 1996. Chronic electrical stimulation of the ventralis intermedius nucleus of the thalamus as a treatment of movement disorders. J Neurosurg 84:203-14

Bence NF, Sampat RM, Kopito RR. 2001. Impairment of the ubiquitin-proteasome system by protein aggregation. Science 292:1552-5

Bennett MC, Bishop JF, Leng Y, Chock PB, Chase TN, Mouradian MM. 1999.

Degradation of alpha-synuclein by proteasome. J Biol Chem 274:33855-8

Berger Y, Blaivas JG, DeLaRocha ER, Salinas JM. 1987. Urodynamic findings in Parkinson's disease. J Urol 138:836-8

Bernardi P, Scorrano L, Colonna R, Petronilli V, Di Lisa F. 1999. Mitochondria and cell death. Mechanistic aspects and methodological issues. Eur J Biochem 264:687-701

Bernheimer H, Birkmayer W, Hornykiewicz O, Jellinger K, Seitelberger F. 1973. Brain dopamine and the syndromes of Parkinson and Huntington. Clinical, morphological and neurochemical correlations. J Neurol Sci 20:415-55

Betarbet R, Sherer TB, MacKenzie G, Garcia-Osuna M, Panov AV, Greenamyre JT. 2000. Chronic systemic pesticide exposure reproduces features of Parkinson's disease. Nat Neurosci 3:1301-6

Beyer MK, Herlofson K, Arsland D, Larsen JP. 2001. Causes of death in a communitybased study of Parkinson's disease. Acta Neurol Scand 103:7-11

Bharucha NE, Bharucha EP, Bharucha AE, Bhise AV, Schoenberg BS. 1988.

Prevalence of Parkinson's disease in the Parsi community of Bombay, India. Arch Neurol 45:1321-3

Block G, Hartman AM. 1989. Issues in reproducibility and validity of dietary studies. Am J Clin Nutr 50:1133-8; discussion 231-5

Bon MA, Jansen Steur EN, de Vos RA, Vermes I. 1999. Neurogenetic correlates of Parkinson's disease: apolipoprotein-E and cytochrome P450 2D6 genetic polymorphism. Neurosci Lett 266:149-51

Bonifati V, Rizzu P, van Baren MJ, Schaap O, Breedveld GJ, et al. 2003. Mutations in the DJ-1 gene associated with autosomal recessive early-onset parkinsonism. Science 299:256-9

Booth RG, Castagnoli N, Jr., Rollema H. 1989. Intracerebral microdialysis neurotoxicity studies of quinoline and isoquinoline derivatives related to MPTP/MPP+. Neurosci Lett 100:306-12

Braak H, de Vos RA, Bohl J, Del Tredici K. 2006. Gastric alpha-synuclein immunoreactive inclusions in Meissner's and Auerbach's plexuses in cases staged for Parkinson's disease-related brain pathology. Neurosci Lett 396:67-72 
Brenner C, Kroemer G. 2000. Apoptosis. Mitochondria--the death signal integrators. Science 289:1150-1

Brown RG, Marsden CD. 1988. 'Subcortical dementia': the neuropsychological evidence. Neuroscience 25:363-87

Butterfield PG, Valanis BG, Spencer PS, Lindeman CA, Nutt JG. 1993. Environmental antecedents of young-onset Parkinson's disease. Neurology 43:1150-8

Buzzard IM, Stanton CA, Figueiredo M, Fries EA, Nicholson R, et al. 2001. Development and reproducibility of a brief food frequency questionnaire for assessing the fat, fiber, and fruit and vegetable intakes of rural adolescents. J Am Diet Assoc 101:1438-46

Cabin DE, Shimazu K, Murphy D, Cole NB, Gottschalk W, et al. 2002. Synaptic vesicle depletion correlates with attenuated synaptic responses to prolonged repetitive stimulation in mice lacking alpha-synuclein. J Neurosci 22:8797-807

Calne DB, Chu NS, Huang CC, Lu CS, Olanow W. 1994. Manganism and idiopathic parkinsonism: similarities and differences. Neurology 44:1583-6

Caparros-Lefebvre D, Sergeant N, Lees A, Camuzat A, Daniel S, et al. 2002.

Guadeloupean parkinsonism: a cluster of progressive supranuclear palsy-like tauopathy. Brain 125:801-11

Casson IR, Siegel O, Sham R, Campbell EA, Tarlau M, DiDomenico A. 1984. Brain damage in modern boxers. JAMA 251:2663-7

Caudle WM, Richardson JR, Wang MZ, Taylor TN, Guillot TS, et al. 2007. Reduced vesicular storage of dopamine causes progressive nigrostriatal neurodegeneration. $J$ Neurosci 27:8138-48

Chai J, Du C, Wu JW, Kyin S, Wang X, Shi Y. 2000. Structural and biochemical basis of apoptotic activation by Smac/DIABLO. Nature 406:855-62

Chandra S, Fornai F, Kwon HB, Yazdani U, Atasoy D, et al. 2004. Double-knockout mice for alpha- and beta-synucleins: effect on synaptic functions. Proc Natl Acad Sci U S A 101:14966-71

Charcot J-M. 1872-1873. Lecons sur les Maladies du Systeme Nerveux. pp. 439-67. Paris: Adrien Delahaye

Chun HS, Gibson GE, DeGiorgio LA, Zhang H, Kidd VJ, Son JH. 2001. Dopaminergic cell death induced by MPP(+), oxidant and specific neurotoxicants shares the common molecular mechanism. $J$ Neurochem 76:1010-21

Clark DE. 1999a. Rapid calculation of polar molecular surface area and its application to the prediction of transport phenomena. 1. Prediction of intestinal absorption. J Pharm Sci 88:807-14 
Clark DE. 1999b. Rapid calculation of polar molecular surface area and its application to the prediction of transport phenomena. 2. Prediction of blood-brain barrier penetration. $J$ Pharm Sci 88:815-21

Clark IE, Dodson MW, Jiang C, Cao JH, Huh JR, et al. 2006. Drosophila pink1 is required for mitochondrial function and interacts genetically with parkin. Nature 441:1162-6

Clark LN, Nicolai A, Afridi S, Harris J, Mejia-Santana H, et al. 2005. Pilot association study of the beta-glucocerebrosidase N370S allele and Parkinson's disease in subjects of Jewish ethnicity. Mov Disord 20:100-3

Clayton DF, George JM. 1998. The synucleins: a family of proteins involved in synaptic function, plasticity, neurodegeneration and disease. Trends Neurosci 21:249-54

Cody FW, MacDermott N, Matthews PB, Richardson HC. 1986. Observations on the genesis of the stretch reflex in Parkinson's disease. Brain 109 (Pt 2):229-49

Cohen G, Collins M. 1970. Alkaloids from catecholamines in adrenal tissue: possible role in alcoholism. Science 167:1749-51

Cohen G, Mytilineou G, Barrett RE. 1972. 6,7-Dihydroxytetrahydroisoquinoline: uptake and storage by peripheral sympathetic nerve of the rat. Science 175:1269-72

Colebrooke RE, Humby T, Lynch PJ, McGowan DP, Xia J, Emson PC. 2006. Agerelated decline in striatal dopamine content and motor performance occurs in the absence of nigral cell loss in a genetic mouse model of Parkinson's disease. Eur $\mathrm{J}$ Neurosci 24:2622-30

Collins AC, Cashaw JL, Davis VE. 1973. Dopamine-derived tetrahydroisoquinoline alkaloids--inhibitors of neuroamine metabolism. Biochem Pharmacol 22:2337-48

Collins MA. 2002. Alkaloids, alcohol and Parkinson's disease. Parkinsonism Relat Disord 8:417-22

Collins MA, Origitano TC. 1983. Catecholamine-derived tetrahydroisoquinolines: Omethylation patterns and regional brain distribution following intraventricular administration in rats. $J$ Neurochem 41:1569-75

Collins MA, Ung-Chhun N, Cheng BY, Pronger D. 1990. Brain and plasma tetrahydroisoquinolines in rats: effects of chronic ethanol intake and diet. $J$ Neurochem 55:1507-14

Conway KA, Harper JD, Lansbury PT. 1998. Accelerated in vitro fibril formation by a mutant alpha-synuclein linked to early-onset Parkinson disease. Nat Med 4:1318-20

Conway KA, Lee SJ, Rochet JC, Ding TT, Williamson RE, Lansbury PT, Jr. 2000. Acceleration of oligomerization, not fibrillization, is a shared property of both alphasynuclein mutations linked to early-onset Parkinson's disease: implications for pathogenesis and therapy. Proc Natl Acad Sci U S A 97:571-6 
Cuervo AM, Stefanis L, Fredenburg R, Lansbury PT, Sulzer D. 2004. Impaired degradation of mutant alpha-synuclein by chaperone-mediated autophagy. Science 305:1292-5

Cummings MC, Winterford CM, Walker NI. 1997. Apoptosis. Am J Surg Pathol 21:88101

D'Amato RJ, Zweig RM, Whitehouse PJ, Wenk GL, Singer HS, et al. 1987. Aminergic systems in Alzheimer's disease and Parkinson's disease. Ann Neurol 22:229-36

Dauer W, Kholodilov N, Vila M, Trillat AC, Goodchild R, et al. 2002. Resistance of alpha -synuclein null mice to the parkinsonian neurotoxin MPTP. Proc Natl Acad Sci U S A 99:14524-9

Davies KN, King D, Davies H. 1994. A study of the nutritional status of elderly patients with Parkinson's disease. Age Ageing 23:142-5

Dawson TM, Dawson VL. 2003. Molecular pathways of neurodegeneration in Parkinson's disease. Science 302:819-22

Day BL, Dick JP, Marsden CD. 1984. Patients with Parkinson's disease can employ a predictive motor strategy. J Neurol Neurosurg Psychiatry 47:1299-306

DeCuypere M, Kalabokis VN, Hao R, Schroeder D, Miller DD, LeDoux MS. 2008a. Localization of $\mathrm{N}$-methyl-norsalsolinol within rodent and human brain. $J$ Neurosci Res 86:2543-52

DeCuypere M, Lu Y, Miller DD, LeDoux MS. 2008b. Regional distribution of tetrahydroisoquinoline derivatives in rodent, human, and Parkinson's disease brain. $J$ Neurochem 107:1398-413

Den Hartog Jager WA, Bethlem J. 1960. The distribution of Lewy bodies in the central and autonomic nervous systems in idiopathic paralysis agitans. $J$ Neurol Neurosurg Psychiatry 23:283-90

Deng H, Dodson MW, Huang H, Guo M. 2008. The Parkinson's disease genes pink1 and parkin promote mitochondrial fission and/or inhibit fusion in Drosophila. Proc Natl Acad Sci U S A 105:14503-8

Deng Y, Maruyama W, Kawai M, Dostert P, Yamamura H, et al. 1997. Assay for the (R)and (S)-enantiomers of salsolinols in biological samples and foods with ion-pair highperformance liquid chromatography using beta-cyclodextrin as a chiral mobile phase additive. J Chromatogr B Biomed Sci Appl 689:313-20

Dennison BA, Jenkins PL, Rockwell HL. 2000. Development and validation of an instrument to assess child dietary fat intake. Prev Med 31:214-24

Dexter DT, Carayon A, Javoy-Agid F, Agid Y, Wells FR, et al. 1991. Alterations in the levels of iron, ferritin and other trace metals in Parkinson's disease and other neurodegenerative diseases affecting the basal ganglia. Brain 114 ( Pt 4):1953-75 
Dexter DT, Carter CJ, Wells FR, Javoy-Agid F, Agid Y, et al. 1989. Basal lipid peroxidation in substantia nigra is increased in Parkinson's disease. $J$ Neurochem $52: 381-9$

Diamond SG, Markham CH. 1976. Present morality in parkinson's disease: the ratio of observed to expected deaths with a method to calculate expected deaths. J Neural Transm 38:259-69

Diamond SG, Markham CH, Hoehn MM, McDowell FH, Muenter MD. 1990. An examination of male-female differences in progression and mortality of Parkinson's disease. Neurology 40:763-6

Dodson MW, Guo M. 2007. Pink1, Parkin, DJ-1 and mitochondrial dysfunction in Parkinson's disease. Curr Opin Neurobiol 17:331-7

Dostert P, Strolin Benedetti M, Dordain G, Vernay D. 1989. Enantiomeric composition of urinary salsolinol in parkinsonian patients after Madopar. J Neural Transm Park Dis Dement Sect 1:269-78

Doty RL, Stern MB, Pfeiffer C, Gollomp SM, Hurtig HI. 1992. Bilateral olfactory dysfunction in early stage treated and untreated idiopathic Parkinson's disease. $J$ Neurol Neurosurg Psychiatry 55:138-42

Duda JE, Giasson BI, Mabon ME, Lee VM, Trojanowski JQ. 2002. Novel antibodies to synuclein show abundant striatal pathology in Lewy body diseases. Ann Neurol 52:20510

Duncan MW, Smythe GA. 1982. Salsolinol and dopamine in alcoholic beverages. Lancet 1:904-5

Duncan MW, Smythe GA, Nicholson MV, Clezy PS. 1984. Comparison of highperformance liquid chromatography with electrochemical detection and gas chromatography-mass fragmentography for the assay of salsolinol, dopamine and dopamine metabolites in food and beverage samples. $J$ Chromatogr 336:199-209

Duvoisin RC, Golbe LI. 1989. Toward a definition of Parkinson's disease. Neurology 39:746

Duvoisin RC, Yahr MD. 1965. Encephalitis and Parkinsonism. Arch Neurol 12:227-39

Edwards LL, Quigley EM, Pfeiffer RF. 1992. Gastrointestinal dysfunction in Parkinson's disease: frequency and pathophysiology. Neurology 42:726-32

Ekstrand MI, Terzioglu M, Galter D, Zhu S, Hofstetter C, et al. 2007. Progressive parkinsonism in mice with respiratory-chain-deficient dopamine neurons. Proc Natl Acad Sci U S A 104:1325-30

Elbaz A, Bower JH, Peterson BJ, Maraganore DM, McDonnell SK, et al. 2003. Survival study of Parkinson disease in Olmsted County, Minnesota. Arch Neurol 60:91-6 
Eriksen JL, Dawson TM, Dickson DW, Petrucelli L. 2003. Caught in the act: alphasynuclein is the culprit in Parkinson's disease. Neuron 40:453-6

Eriksen JL, Wszolek Z, Petrucelli L. 2005. Molecular pathogenesis of Parkinson disease. Arch Neurol 62:353-7

Ertl P, Rohde B, Selzer P. 2000. Fast calculation of molecular polar surface area as a sum of fragment-based contributions and its application to the prediction of drug transport properties. J Med Chem 43:3714-7

Esposito LA, Melov S, Panov A, Cottrell BA, Wallace DC. 1999. Mitochondrial disease in mouse results in increased oxidative stress. Proc Natl Acad Sci U S A 96:4820-5

Evans MJ, Scarpulla RC. 1990. NRF-1: a trans-activator of nuclear-encoded respiratory genes in animal cells. Genes Dev 4:1023-34

Fahn S. 2003. Description of Parkinson's disease as a clinical syndrome. Ann N Y Acad Sci $991: 1-14$

Fall PA, Fredrikson M, Axelson O, Granerus AK. 1999. Nutritional and occupational factors influencing the risk of Parkinson's disease: a case-control study in southeastern Sweden. Mov Disord 14:28-37

Feany MB, Bender WW. 2000. A Drosophila model of Parkinson's disease. Nature 404:394-8

Fearnley JM, Lees AJ. 1991. Ageing and Parkinson's disease: substantia nigra regional selectivity. Brain 114 (Pt 5):2283-301

Findley LJ, Gresty MA, Halmagyi GM. 1981. Tremor, the cogwheel phenomenon and clonus in Parkinson's disease. J Neurol Neurosurg Psychiatry 44:534-46

Fleming SM, Salcedo J, Hutson CB, Rockenstein E, Masliah E, et al. 2006. Behavioral effects of dopaminergic agonists in transgenic mice overexpressing human wildtype alpha-synuclein. Neuroscience 142:1245-53

Fornai F, Lenzi P, Gesi M, Ferrucci M, Lazzeri G, et al. 2003. Fine structure and biochemical mechanisms underlying nigrostriatal inclusions and cell death after proteasome inhibition. J Neurosci 23:8955-66

Fornai F, Schluter OM, Lenzi P, Gesi M, Ruffoli R, et al. 2005. Parkinson-like syndrome induced by continuous MPTP infusion: convergent roles of the ubiquitin-proteasome system and alpha-synuclein. Proc Natl Acad Sci U S A 102:3413-8

Forno LS. 1996. Neuropathology of Parkinson's disease. J Neuropathol Exp Neurol $55: 259-72$

Forno LS, Sternberger LA, Sternberger NH, Strefling AM, Swanson K, Eng LF. 1986. Reaction of Lewy bodies with antibodies to phosphorylated and non-phosphorylated neurofilaments. Neurosci Lett 64:253-8 
Fountaine TM, Venda LL, Warrick N, Christian HC, Brundin P, et al. 2008. The effect of alpha-synuclein knockdown on MPP+ toxicity in models of human neurons. Eur $\mathrm{J}$ Neurosci 28:2459-73

Fountaine TM, Wade-Martins R. 2007. RNA interference-mediated knockdown of alphasynuclein protects human dopaminergic neuroblastoma cells from MPP $(+)$ toxicity and reduces dopamine transport. $J$ Neurosci Res 85:351-63

Fratiglioni L, Wang HX. 2000. Smoking and Parkinson's and Alzheimer's disease: review of the epidemiological studies. Behav Brain Res 113:117-20

Fukuda T. 1994. 2-Methyl-1,2,3,4-tetrahydroisoquinoline does dependently reduce the number of tyrosine hydroxylase-immunoreactive cells in the substantia nigra and locus ceruleus of C57BL/6J mice. Brain Res 639:325-8

Funayama M, Hasegawa K, Kowa H, Saito M, Tsuji S, Obata F. 2002. A new locus for Parkinson's disease (PARK8) maps to chromosome 12p11.2-q13.1. Ann Neurol 51:296301

Gai WP, Yuan HX, Li XQ, Power JT, Blumbergs PC, Jensen PH. 2000. In situ and in vitro study of colocalization and segregation of alpha-synuclein, ubiquitin, and lipids in Lewy bodies. Exp Neurol 166:324-33

Gainetdinov RR, Fumagalli F, Jones SR, Caron MG. 1997. Dopamine transporter is required for in vivo MPTP neurotoxicity: evidence from mice lacking the transporter. $J$ Neurochem 69:1322-5

Galvin JE, Lee VM, Baba M, Mann DM, Dickson DW, et al. 1997. Monoclonal antibodies to purified cortical Lewy bodies recognize the mid-size neurofilament subunit. Ann Neurol 42:595-603

Galvin JE, Lee VM, Schmidt ML, Tu PH, Iwatsubo T, Trojanowski JQ. 1999. Pathobiology of the Lewy body. Adv Neurol 80:313-24

Gandhi S, Muqit MM, Stanyer L, Healy DG, Abou-Sleiman PM, et al. 2006. PINK1 protein in normal human brain and Parkinson's disease. Brain 129:1720-31

Gandhi S, Wood NW. 2005. Molecular pathogenesis of Parkinson's disease. Hum Mol Genet 14:2749-55

Gao X, Wilde PE, Lichtenstein AH, Bermudez OI, Tucker KL. 2006. The maximal amount of dietary alpha-tocopherol intake in U.S. adults (NHANES 2001-2002). J Nutr 136:1021-6

Gasser T, Muller-Myhsok B, Wszolek ZK, Oehlmann R, Calne DB, et al. 1998. A susceptibility locus for Parkinson's disease maps to chromosome 2p13. Nat Genet $18: 262-5$

Gautier CA, Kitada T, Shen J. 2008. Loss of PINK1 causes mitochondrial functional defects and increased sensitivity to oxidative stress. Proc Natl Acad Sci U S A 105:11364-9 
Geddes JW. 2005. alpha-Synuclein: a potent inducer of tau pathology. Exp Neurol 192:244-50

Giasson BI, Forman MS, Higuchi M, Golbe LI, Graves CL, et al. 2003. Initiation and synergistic fibrillization of tau and alpha-synuclein. Science 300:636-40

Giasson BI, Uryu K, Trojanowski JQ, Lee VM. 1999. Mutant and wild type human alphasynucleins assemble into elongated filaments with distinct morphologies in vitro. $J$ Biol Chem 274:7619-22

Gibb WR, Lees AJ. 1988. The relevance of the Lewy body to the pathogenesis of idiopathic Parkinson's disease. J Neurol Neurosurg Psychiatry 51:745-52

Gibberd FB, Gleeson JA, Gossage AA, Wilson RS. 1974. Oesophageal dilatation in Parkinson's disease. J Neurol Neurosurg Psychiatry 37:938-40

Goker-Alpan O, Schiffmann R, LaMarca ME, Nussbaum RL, Mclnerney-Leo A, Sidransky E. 2004. Parkinsonism among Gaucher disease carriers. J Med Genet 41:937-40

Golbe LI, Farrell TM, Davis PH. 1988. Case-control study of early life dietary factors in Parkinson's disease. Arch Neurol 45:1350-3

Goldberg MS, Fleming SM, Palacino JJ, Cepeda C, Lam HA, et al. 2003. Parkindeficient mice exhibit nigrostriatal deficits but not loss of dopaminergic neurons. J Biol Chem 278:43628-35

Goldman JE, Yen SH. 1986. Cytoskeletal protein abnormalities in neurodegenerative diseases. Ann Neurol 19:209-23

Goldman JE, Yen SH, Chiu FC, Peress NS. 1983. Lewy bodies of Parkinson's disease contain neurofilament antigens. Science 221:1082-4

Gotham AM, Brown RG, Marsden CD. 1986. Depression in Parkinson's disease: a quantitative and qualitative analysis. J Neurol Neurosurg Psychiatry 49:381-9

Grandinetti A, Morens DM, Reed D, MacEachern D. 1994. Prospective study of cigarette smoking and the risk of developing idiopathic Parkinson's disease. Am J Epidemiol 139:1129-38

Green DR. 2005. Apoptotic pathways: ten minutes to dead. Cell 121:671-4

Green DR, Kroemer G. 2004. The pathophysiology of mitochondrial cell death. Science 305:626-9

Greenamyre JT, Hastings TG. 2004. Biomedicine. Parkinson's--divergent causes, convergent mechanisms. Science 304:1120-2

Guekht A, Selikhova M, Serkin G, Gusev E. 2005. Implementation of the TMS in the early stages of Parkinson's disease. Electromyogr Clin Neurophysiol 45:291-7 
Hald A, Lotharius J. 2005. Oxidative stress and inflammation in Parkinson's disease: is there a causal link? Exp Neurol 193:279-90

Hamill RW, Caine E, Eskin T, Lapham L, Shoulson I, McNeill TH. 1988.

Neurodegenerative disorders and aging. Alzheimer's disease and Parkinson's disease-common ground. Ann N Y Acad Sci 515:411-20

Hampshire DJ, Roberts E, Crow Y, Bond J, Mubaidin A, et al. 2001. Kufor-Rakeb syndrome, pallido-pyramidal degeneration with supranuclear upgaze paresis and dementia, maps to 1p36. J Med Genet 38:680-2

Hansen L, Salmon D, Galasko D, Masliah E, Katzman R, et al. 1990. The Lewy body variant of Alzheimer's disease: a clinical and pathologic entity. Neurology 40:1-8

Hansen LA, Samuel W. 1997. Criteria for Alzheimer's disease and the nosology of dementia with Lewy bodies. Neurology 48:126-32

Harada H, Nishikawa S, Takahashi K. 1983. Epidemiology of Parkinson's disease in a Japanese city. Arch Neurol 40:151-4

Hashimoto M, Hsu LJ, Sisk A, Xia Y, Takeda A, et al. 1998. Human recombinant NACP/alpha-synuclein is aggregated and fibrillated in vitro: relevance for Lewy body disease. Brain Res 799:301-6

Hashimoto M, Hsu LJ, Xia Y, Takeda A, Sisk A, et al. 1999. Oxidative stress induces amyloid-like aggregate formation of NACP/alpha-synuclein in vitro. Neuroreport 10:71721

Hayashida K, Oyanagi S, Mizutani Y, Yokochi M. 1993. An early cytoplasmic change before Lewy body maturation: an ultrastructural study of the substantia nigra from an autopsy case of juvenile parkinsonism. Acta Neuropathol 85:445-8

Hellenbrand W, Boeing H, Robra BP, Seidler A, Vieregge P, et al. 1996a. Diet and Parkinson's disease. II: A possible role for the past intake of specific nutrients. Results from a self-administered food-frequency questionnaire in a case-control study. Neurology 47:644-50

Hellenbrand W, Seidler A, Boeing H, Robra BP, Vieregge P, et al. 1996b. Diet and Parkinson's disease. I: A possible role for the past intake of specific foods and food groups. Results from a self-administered food-frequency questionnaire in a case-control study. Neurology 47:636-43

Hely MA, Morris JG, Reid WG, Trafficante R. 2005. Sydney Multicenter Study of Parkinson's disease: non-L-dopa-responsive problems dominate at 15 years. Mov Disord 20:190-9

Herlofson K, Lie SA, Arsland D, Larsen JP. 2004. Mortality and Parkinson disease: a community based study. Neurology 62:937-42

Hermanson GT. 1996. Bioconjugate Techniques. San Diego: Academic Press 
Hertzman C, Wiens M, Bowering D, Snow B, Calne D. 1990. Parkinson's disease: a case-control study of occupational and environmental risk factors. Am J Ind Med 17:34955

Hill WD, Lee VM, Hurtig HI, Murray JM, Trojanowski JQ. 1991. Epitopes located in spatially separate domains of each neurofilament subunit are present in Parkinson's disease Lewy bodies. J Comp Neurol 309:150-60

Hirata Y, Nagatsu T. 1985. Inhibition of tyrosine hydroxylation in tissue slices of the rat striatum by 1-methyl-4-phenyl-1,2,3,6-tetrahydropyridine. Brain Res 337:193-6

Hirsch EC, Faucheux BA. 1998. Iron metabolism and Parkinson's disease. Mov Disord 13 Suppl 1:39-45

Hoehn MM, Yahr MD. 1967. Parkinsonism: onset, progression and mortality. Neurology $17: 427-42$

Hoelscher DM, Day RS, Kelder SH, Ward JL. 2003. Reproducibility and validity of the secondary level School-Based Nutrition Monitoring student questionnaire. J Am Diet Assoc 103:186-94

Hofman A, Collette HJ, Bartelds Al. 1989. Incidence and risk factors of Parkinson's disease in The Netherlands. Neuroepidemiology 8:296-9

Hoglinger GU, Carrard G, Michel PP, Medja F, Lombes A, et al. 2003. Dysfunction of mitochondrial complex I and the proteasome: interactions between two biochemical deficits in a cellular model of Parkinson's disease. J Neurochem 86:1297-307

Holm S. 1979. A simple sequentially rejective multiple test procedure. Scandinavian Journal of Statistics 6:65-70

Honig LS, Rosenberg RN. 2000. Apoptosis and neurologic disease. Am J Med 108:31730

Hsu LJ, Sagara Y, Arroyo A, Rockenstein E, Sisk A, et al. 2000. alpha-synuclein promotes mitochondrial deficit and oxidative stress. Am J Pathol 157:401-10

Hu FB. 2002. Dietary pattern analysis: a new direction in nutritional epidemiology. Curr Opin Lipidol 13:3-9

Hubble JP, Cao T, Hassanein RE, Neuberger JS, Koller WC. 1993. Risk factors for Parkinson's disease. Neurology 43:1693-7

Hughes AJ, Ben-Shlomo Y, Daniel SE, Lees AJ. 1992. What features improve the accuracy of clinical diagnosis in Parkinson's disease: a clinicopathologic study. Neurology 42:1142-6

Hughes AJ, Daniel SE, Blankson S, Lees AJ. 1993. A clinicopathologic study of 100 cases of Parkinson's disease. Arch Neurol 50:140-8 
Hurtig HI. 1997. Problems with current pharmacologic treatment of Parkinson's disease. Exp Neurol 144:10-6

Irizarry MC, Growdon W, Gomez-Isla T, Newell K, George JM, et al. 1998. Nigral and cortical Lewy bodies and dystrophic nigral neurites in Parkinson's disease and cortical Lewy body disease contain alpha-synuclein immunoreactivity. J Neuropathol Exp Neurol 57:334-7

Jacques PF, Tucker KL. 2001. Are dietary patterns useful for understanding the role of diet in chronic disease? Am J Clin Nutr 73:1-2

James AM, Wei YH, Pang CY, Murphy MP. 1996. Altered mitochondrial function in fibroblasts containing MELAS or MERRF mitochondrial DNA mutations. Biochem J 318 (Pt 2):401-7

Jenkins IH, Fernandez W, Playford ED, Lees AJ, Frackowiak RS, et al. 1992. Impaired activation of the supplementary motor area in Parkinson's disease is reversed when akinesia is treated with apomorphine. Ann Neurol 32:749-57

Johnson CC, Gorell JM, Rybicki BA, Sanders K, Peterson EL. 1999. Adult nutrient intake as a risk factor for Parkinson's disease. Int J Epidemiol 28:1102-9

Jost WH. 2010. Gastrointestinal dysfunction in Parkinson's Disease. J Neurol Sci 289:69-73

Joza N, Susin SA, Daugas E, Stanford WL, Cho SK, et al. 2001. Essential role of the mitochondrial apoptosis-inducing factor in programmed cell death. Nature 410:549-54

Kajita M, Niwa T, Maruyama W, Nakahara D, Takeda N, et al. 1994. Endogenous synthesis of $\mathrm{N}$-methylnorsalsolinol in rat brain during in vivo microdialysis with epinine. $J$ Chromatogr B Biomed Appl 654:263-9

Kawai H, Makino Y, Hirobe M, Ohta S. 1998. Novel endogenous 1,2,3,4tetrahydroisoquinoline derivatives: uptake by dopamine transporter and activity to induce parkinsonism. J Neurochem 70:745-51

Kessler, II. 1972. Epidemiologic studies of Parkinson's disease. 3. A community-based survey. Am J Epidemiol 96:242-54

Kikuchi K, Nagatsu Y, Makino Y, Mashino T, Ohta S, Hirobe M. 1991. Metabolism and penetration through blood-brain barrier of parkinsonism-related compounds. 1,2,3,4Tetrahydroisoquinoline and 1-methyl-1,2,3,4-tetrahydroisoquinoline. Drug Metab Dispos 19:257-62

Kirik D, Annett LE, Burger C, Muzyczka N, Mandel RJ, Bjorklund A. 2003. Nigrostriatal alpha-synucleinopathy induced by viral vector-mediated overexpression of human alphasynuclein: a new primate model of Parkinson's disease. Proc Natl Acad Sci U S A 100:2884-9 
Kish SJ, Shannak K, Hornykiewicz O. 1988. Uneven pattern of dopamine loss in the striatum of patients with idiopathic Parkinson's disease. Pathophysiologic and clinical implications. N Engl J Med 318:876-80

Kitada T, Asakawa S, Hattori N, Matsumine H, Yamamura Y, et al. 1998. Mutations in the parkin gene cause autosomal recessive juvenile parkinsonism. Nature 392:605-8

Kobayashi H, Fukuhara K, Tada-Oikawa S, Yada Y, Hiraku Y, et al. 2009. The mechanisms of oxidative DNA damage and apoptosis induced by norsalsolinol, an endogenous tetrahydroisoquinoline derivative associated with Parkinson's disease. $J$ Neurochem 108:397-407

Kohno M, Ohta S, Hirobe M. 1986. Tetrahydroisoquinoline and 1-methyltetrahydroisoquinoline as novel endogenous amines in rat brain. Biochem Biophys Res Commun 140:448-54

Koller W, Vetere-Overfield B, Gray C, Alexander C, Chin T, et al. 1990. Environmental risk factors in Parkinson's disease. Neurology 40:1218-21

Konradi C, Kornhuber J, Sofic E, Heckers S, Riederer P, Beckmann H. 1992. Variations of monoamines and their metabolites in the human brain putamen. Brain Res 579:28590

Kotake Y, Ohta S. 2003. MPP+ analogs acting on mitochondria and inducing neurodegeneration. Curr Med Chem 10:2507-16

Kotake Y, Ohta S, Kanazawa I, Sakurai M. 2003. Neurotoxicity of an endogenous brain amine, 1-benzyl-1,2,3,4-tetrahydroisoquinoline, in organotypic slice co-culture of mesencephalon and striatum. Neuroscience 117:63-70

Kotake Y, Sekiya Y, Okuda K, Ohta S. 2007. Cytotoxicity of 17 tetrahydroisoquinoline derivatives in SH-SY5Y human neuroblastoma cells is related to mitochondrial NADHubiquinone oxidoreductase inhibition. Neurotoxicology 28:27-32

Kotake Y, Tasaki Y, Makino Y, Ohta S, Hirobe M. 1995. 1-Benzyl-1,2,3,4tetrahydroisoquinoline as a parkinsonism-inducing agent: a novel endogenous amine in mouse brain and parkinsonian CSF. $J$ Neurochem 65:2633-8

Kotake Y, Yoshida M, Ogawa M, Tasaki Y, Hirobe M, Ohta S. 1996. Chronic administration of 1-benzyl-1,2,3,4-tetrahydroisoquinoline, an endogenous amine in the brain, induces parkinsonism in a primate. Neurosci Lett 217:69-71

Kotzbauer PT, Giasson BI, Kravitz AV, Golbe LI, Mark MH, et al. 2004. Fibrillization of alpha-synuclein and tau in familial Parkinson's disease caused by the A53T alphasynuclein mutation. Exp Neurol 187:279-88

Kroemer G. 1999. Mitochondrial control of apoptosis: an overview. Biochem Soc Symp $66: 1-15$

Kurz M, Alves G, Aarsland D, Larsen JP. 2003. Familial Parkinson's disease: a community-based study. Eur J Neurol 10:159-63 
Kuzuhara S, Mori H, Izumiyama N, Yoshimura M, Ihara Y. 1988. Lewy bodies are ubiquitinated. A light and electron microscopic immunocytochemical study. Acta Neuropathol 75:345-53

Laitinen LV, Bergenheim AT, Hariz MI. 1992. Ventroposterolateral pallidotomy can abolish all parkinsonian symptoms. Stereotact Funct Neurosurg 58:14-21

Lakso M, Vartiainen S, Moilanen AM, Sirvio J, Thomas JH, et al. 2003. Dopaminergic neuronal loss and motor deficits in Caenorhabditis elegans overexpressing human alpha-synuclein. J Neurochem 86:165-72

Lance JW, Schwab RS, Peterson EA. 1963. Action tremor and the cogwheel phenomenon in Parkinson's disease. Brain 86:95-110

Langston JW, Ballard P, Tetrud JW, Irwin I. 1983. Chronic Parkinsonism in humans due to a product of meperidine-analog synthesis. Science 219:979-80

Lannuzel A, Michel PP, Caparros-Lefebvre D, Abaul J, Hocquemiller R, Ruberg M. 2002. Toxicity of Annonaceae for dopaminergic neurons: potential role in atypical parkinsonism in Guadeloupe. Mov Disord 17:84-90

Lannuzel A, Michel PP, Hoglinger GU, Champy P, Jousset A, et al. 2003. The mitochondrial complex I inhibitor annonacin is toxic to mesencephalic dopaminergic neurons by impairment of energy metabolism. Neuroscience 121:287-96

Lardy HA, Ferguson SM. 1969. Oxidative phosphorylation in mitochondria. Annu Rev Biochem 38:991-1034

Lee CS, Han ES, Park ES, Bang H. 2005. Inhibition of MG132-induced mitochondrial dysfunction and cell death in PC12 cells by 3-morpholinosydnonimine. Brain Res 1036:18-26

Lee HJ, Khoshaghideh F, Patel S, Lee SJ. 2004a. Clearance of alpha-synuclein oligomeric intermediates via the lysosomal degradation pathway. $J$ Neurosci 24:1888-96

Lee VM, Giasson BI, Trojanowski JQ. 2004b. More than just two peas in a pod: common amyloidogenic properties of tau and alpha-synuclein in neurodegenerative diseases. Trends Neurosci 27:129-34

Leroy E, Anastasopoulos D, Konitsiotis S, Lavedan C, Polymeropoulos MH. 1998. Deletions in the Parkin gene and genetic heterogeneity in a Greek family with early onset Parkinson's disease. Hum Genet 103:424-7

Levy G, Khanna NN, Soda DM, Tsuzuki O, Stern L. 1975. Pharmacokinetics of acetaminophen in the human neonate: formation of acetaminophen glucuronide and sulfate in relation to plasma bilirubin concentration and D-glucaric acid excretion. Pediatrics 55:818-25

Li SC, Schoenberg BS, Wang CC, Cheng XM, Rui DY, et al. 1985. A prevalence survey of Parkinson's disease and other movement disorders in the People's Republic of China. Arch Neurol 42:655-7 
Lippa CF, Fujiwara H, Mann DM, Giasson B, Baba M, et al. 1998. Lewy bodies contain altered alpha-synuclein in brains of many familial Alzheimer's disease patients with mutations in presenilin and amyloid precursor protein genes. Am J Pathol 153:1365-70

Liu S, Ninan I, Antonova I, Battaglia F, Trinchese F, et al. 2004. alpha-Synuclein produces a long-lasting increase in neurotransmitter release. EMBO J 23:4506-16

Logroscino G, Marder K, Cote L, Tang MX, Shea S, Mayeux R. 1996. Dietary lipids and antioxidants in Parkinson's disease: a population-based, case-control study. Ann Neurol 39:89-94

Logroscino G, Marder K, Graziano J, Freyer G, Slavkovich V, et al. 1998. Dietary iron, animal fats, and risk of Parkinson's disease. Mov Disord 13 Suppl 1:13-6

Lowe J, Blanchard A, Morrell K, Lennox G, Reynolds L, et al. 1988. Ubiquitin is a common factor in intermediate filament inclusion bodies of diverse type in man, including those of Parkinson's disease, Pick's disease, and Alzheimer's disease, as well as Rosenthal fibres in cerebellar astrocytomas, cytoplasmic bodies in muscle, and mallory bodies in alcoholic liver disease. J Pathol 155:9-15

Lowe J, McDermott H, Landon M, Mayer RJ, Wilkinson KD. 1990. Ubiquitin carboxylterminal hydrolase (PGP 9.5) is selectively present in ubiquitinated inclusion bodies characteristic of human neurodegenerative diseases. J Pathol 161:153-60

Ludin SM, Ludin HP. 1989. Is Parkinson's disease of early onset a separate disease entity? J Neurol 236:203-7

Luoma PT, Eerola J, Ahola S, Hakonen AH, Hellstrom O, et al. 2007. Mitochondrial DNA polymerase gamma variants in idiopathic sporadic Parkinson disease. Neurology 69:1152-9

Luquin MR, Scipioni O, Vaamonde J, Gershanik O, Obeso JA. 1992. Levodopa-induced dyskinesias in Parkinson's disease: clinical and pharmacological classification. Mov Disord 7:117-24

Lwin A, Orvisky E, Goker-Alpan O, LaMarca ME, Sidransky E. 2004.

Glucocerebrosidase mutations in subjects with parkinsonism. Mol Genet Metab 81:70-3

Makino Y, Ohta S, Tachikawa O, Hirobe M. 1988. Presence of tetrahydroisoquinoline and 1-methyl-tetrahydro-isoquinoline in foods: compounds related to Parkinson's disease. Life Sci 43:373-8

Makino Y, Tasaki Y, Ohta S, Hirobe M. 1990. Confirmation of the enantiomers of 1methyl-1,2,3,4-tetrahydroisoquinoline in the mouse brain and foods applying gas chromatography/mass spectrometry with negative ion chemical ionization. Biomed Environ Mass Spectrom 19:415-9

Mann A, Tyndale RF. 2010. Cytochrome P450 2D6 enzyme neuroprotects against 1methyl-4-phenylpyridinium toxicity in SH-SY5Y neuronal cells. Eur J Neurosci 31:118593 
Mariussen E, Fonnum F. 2001. The effect of polychlorinated biphenyls on the high affinity uptake of the neurotransmitters, dopamine, serotonin, glutamate and GABA, into rat brain synaptosomes. Toxicology 159:11-21

Maroteaux L, Campanelli JT, Scheller RH. 1988. Synuclein: a neuron-specific protein localized to the nucleus and presynaptic nerve terminal. $J$ Neurosci 8:2804-15

Marras C, McDermott MP, Rochon PA, Tanner CM, Naglie G, et al. 2005. Survival in Parkinson disease: thirteen-year follow-up of the DATATOP cohort. Neurology 64:87-93

Marsden CD. 1982. The mysterious motor function of the basal ganglia: the Robert Wartenberg Lecture. Neurology 32:514-39

Martin HF, Gudzinowicz BJ. 1963. Thermal stability of polynuclear aromatic hydrocarbons and the polarographic half-wave reduction potential. Nature 200:69-70

Maruyama W, Abe T, Tohgi H, Naoi M. 1999. An endogenous MPTP-like dopaminergic neurotoxin, $\mathrm{N}$-methyl(R)salsolinol, in the cerebrospinal fluid decreases with progression of Parkinson's disease. Neurosci Lett 262:13-6

Maruyama W, Akao Y, Youdim MB, Davis BA, Naoi M. 2001. Transfection-enforced Bcl2 overexpression and an anti-Parkinson drug, rasagiline, prevent nuclear accumulation of glyceraldehyde-3-phosphate dehydrogenase induced by an endogenous dopaminergic neurotoxin, N-methyl(R)salsolinol. J Neurochem 78:727-35

Maruyama W, Benedetti MS, Takahashi T, Naoi M. 1997a. A neurotoxin Nmethyl(R)salsolinol induces apoptotic cell death in differentiated human dopaminergic neuroblastoma SH-SY5Y cells. Neurosci Lett 232:147-50

Maruyama W, Naoi M, Kasamatsu T, Hashizume Y, Takahashi T, et al. 1997b. An endogenous dopaminergic neurotoxin, $\mathrm{N}$-methyl-(R)-salsolinol, induces DNA damage in human dopaminergic neuroblastoma SH-SY5Y cells. J Neurochem 69:322-9

Maruyama W, Sobue G, Matsubara K, Hashizume Y, Dostert P, Naoi M. 1997c. A dopaminergic neurotoxin, 1(R), 2(N)-dimethyl-6,7-dihydroxy-1,2,3,4-

tetrahydroisoquinoline, $\mathrm{N}$-methyl(R)salsolinol, and its oxidation product, 1,2(N)-dimethyl6,7-dihydroxyisoquinolinium ion, accumulate in the nigro-striatal system of the human brain. Neurosci Lett 223:61-4

Maruyama W, Takahashi T, Minami M, Takahashi A, Dostert P, et al. 1993. Cytotoxicity of dopamine-derived 6,7-dihydroxy-1,2,3,4-tetrahydroisoquinolines. Adv Neurol 60:22430

Maruyama W, Yi H, Takahashi T, Shimazu S, Ohde H, et al. 2004. Neuroprotective function of R-(-)-1-(benzofuran-2-yl)-2-propylaminopentane, [R-(-)-BPAP], against apoptosis induced by $\mathrm{N}$-methyl(R)salsolinol, an endogenous dopaminergic neurotoxin, in human dopaminergic neuroblastoma SH-SY5Y cells. Life Sci 75:107-17

Marzo I, Brenner C, Zamzami N, Jurgensmeier JM, Susin SA, et al. 1998. Bax and adenine nucleotide translocator cooperate in the mitochondrial control of apoptosis. Science 281:2027-31 
Masliah E, Rockenstein E, Veinbergs I, Mallory M, Hashimoto M, et al. 2000.

Dopaminergic loss and inclusion body formation in alpha-synuclein mice: implications for neurodegenerative disorders. Science 287:1265-9

Matthys C, Pynaert I, De Keyzer W, De Henauw S. 2007. Validity and reproducibility of an adolescent web-based food frequency questionnaire. J Am Diet Assoc 107:605-10

Mayeux R, Marder K, Cote LJ, Denaro J, Hemenegildo N, et al. 1995. The frequency of idiopathic Parkinson's disease by age, ethnic group, and sex in northern Manhattan, 1988-1993. Am J Epidemiol 142:820-7

Mayeux R, Stern Y, Cote L, Williams JB. 1984a. Altered serotonin metabolism in depressed patients with Parkinson's disease. Neurology 34:642-6

Mayeux R, Williams JB, Stern Y, Cote L. 1984b. Depression and Parkinson's disease. Adv Neurol 40:241-50

McGeer PL, Itagaki S, Akiyama H, McGeer EG. 1988. Rate of cell death in parkinsonism indicates active neuropathological process. Ann Neurol 24:574-6

McNaught KS, Altomare C, Cellamare S, Carotti A, Thull U, et al. 1995a. Inhibition of alpha-ketoglutarate dehydrogenase by isoquinoline derivatives structurally related to 1methyl-4-phenyl-1,2,3,6-tetrahydropyridine (MPTP). Neuroreport 6:1105-8

McNaught KS, Bjorklund LM, Belizaire R, Isacson O, Jenner P, Olanow CW. 2002. Proteasome inhibition causes nigral degeneration with inclusion bodies in rats. Neuroreport 13:1437-41

McNaught KS, Perl DP, Brownell AL, Olanow CW. 2004. Systemic exposure to proteasome inhibitors causes a progressive model of Parkinson's disease. Ann Neurol $56: 149-62$

McNaught KS, Thull U, Carrupt PA, Altomare C, Cellamare S, et al. 1995b. Inhibition of complex I by isoquinoline derivatives structurally related to 1-methyl-4-phenyl-1,2,3,6tetrahydropyridine (MPTP). Biochem Pharmacol 50:1903-11

Meier P, Finch A, Evan G. 2000. Apoptosis in development. Nature 407:796-801

Meyerson LR, McMurtrey KD, Davis VE. 1976. Neuroamine-derived alkaloids: substratepreferred inhibitors of rat brain monoamine oxidase in vitro. Biochem Pharmacol 25:1013-20

Millen BE, Quatromoni PA, Gagnon DR, Cupples LA, Franz MM, D'Agostino RB. 1996. Dietary patterns of men and women suggest targets for health promotion: the Framingham Nutrition Studies. Am J Health Promot 11:42-52; discussion -3

Minami M, Maruyama W, Dostert P, Nagatsu T, Naoi M. 1993. Inhibition of type A and B monoamine oxidase by 6,7-dihydroxy-1,2,3,4-tetrahydroisoquinolines and their $\mathrm{N}$ methylated derivatives. J Neural Transm Gen Sect 92:125-35 
Mizuno Y, Matuda S, Yoshino H, Mori H, Hattori N, Ikebe S. 1994. An immunohistochemical study on alpha-ketoglutarate dehydrogenase complex in Parkinson's disease. Ann Neurol 35:204-10

Mizuno Y, Yoshino H, Ikebe S, Hattori N, Kobayashi T, et al. 1998. Mitochondrial dysfunction in Parkinson's disease. Ann Neurol 44:S99-109

Molina-Jimenez MF, Sanchez-Reus MI, Benedi J. 2003. Effect of fraxetin and myricetin on rotenone-induced cytotoxicity in SH-SY5Y cells: comparison with $\mathrm{N}$-acetylcysteine. Eur J Pharmacol 472:81-7

Mooslehner KA, Chan PM, Xu W, Liu L, Smadja C, et al. 2001. Mice with very low expression of the vesicular monoamine transporter 2 gene survive into adulthood: potential mouse model for parkinsonism. Mol Cell Biol 21:5321-31

Morens DM, Davis JW, Grandinetti A, Ross GW, Popper JS, White LR. 1996. Epidemiologic observations on Parkinson's disease: incidence and mortality in a prospective study of middle-aged men. Neurology 46:1044-50

Morgante L, Rocca WA, Di Rosa AE, De Domenico P, Grigoletto F, et al. 1992. Prevalence of Parkinson's disease and other types of parkinsonism: a door-to-door survey in three Sicilian municipalities. The Sicilian Neuro-Epidemiologic Study (SNES) Group. Neurology 42:1901-7

Moser A, Kompf D. 1992. Presence of methyl-6,7-dihydroxy-1,2,3,4tetrahydroisoquinolines, derivatives of the neurotoxin isoquinoline, in parkinsonian lumbar CSF. Life Sci 50:1885-91

Moser A, Scholz J, Bamberg H, Bohme V. 1996a. The effect of N-methyl-norsalsolinol on monoamine oxidase of the rat caudate nucleus in vitro. Neurochem Int 28:109-12

Moser A, Scholz J, Nobbe F, Vieregge P, Bohme V, Bamberg H. 1995. Presence of Nmethyl-norsalsolinol in the CSF: correlations with dopamine metabolites of patients with Parkinson's disease. J Neurol Sci 131:183-9

Moser A, Siebecker F, Nobbe F, Bohme V. 1996b. Rotational behaviour and neurochemical changes in unilateral $\mathrm{N}$-methyl-norsalsolinol and 6-hydroxydopamine lesioned rats. Exp Brain Res 112:89-95

Mravec B. 2006. Salsolinol, a derivate of dopamine, is a possible modulator of catecholaminergic transmission: a review of recent developments. Physiol Res 55:35364

Muenter MD, Sharpless NS, Tyce GM, Darley FL. 1977. Patterns of dystonia ("I-D-I" and "D-I-D-") in response to I-dopa therapy for Parkinson's disease. Mayo Clin Proc 52:16374

Muller T, Przuntek H, Kuhn W, Baum SS, Rommelspacher H. 1999. No increase of synthesis of $(\mathrm{R})$ salsolinol in Parkinson's disease. Mov Disord 14:514-5 
Musshoff F, Lachenmeier DW, Kroener L, Schmidt P, Dettmeyer R, Madea B. 2003. Simultaneous gas chromatographic-mass spectrometric determination of dopamine, norsalsolinol and salsolinol enantiomers in brain samples of a large human collective. Cell Mol Biol (Noisy-le-grand) 49:837-49

Musshoff F, Lachenmeier DW, Schmidt P, Dettmeyer R, Madea B. 2005. Systematic regional study of dopamine, norsalsolinol, and (R/S)-salsolinol levels in human brain areas of alcoholics. Alcohol Clin Exp Res 29:46-52

Musshoff F, Schmidt P, Dettmeyer R, Priemer F, Jachau K, Madea B. 2000.

Determination of dopamine and dopamine-derived (R)-/(S)-salsolinol and norsalsolinol in various human brain areas using solid-phase extraction and gas chromatography/mass spectrometry. Forensic Sci Int 113:359-66

Musshoff F, Schmidt P, Dettmeyer R, Priemer F, Wittig H, Madea B. 1999. A systematic regional study of dopamine and dopamine-derived salsolinol and norsalsolinol levels in human brain areas. Forensic Sci Int 105:1-11

Nagatsu T. 1997. Isoquinoline neurotoxins in the brain and Parkinson's disease.

Neurosci Res 29:99-111

Nagatsu T, Yoshida M. 1988. An endogenous substance of the brain, tetrahydroisoquinoline, produces parkinsonism in primates with decreased dopamine, tyrosine hydroxylase and biopterin in the nigrostriatal regions. Neurosci Lett 87:178-82

Naoi M, Maruyama W, Akao Y, Yi H. 2002. Dopamine-derived endogenous N-methyl(R)-salsolinol: its role in Parkinson's disease. Neurotoxicol Teratol 24:579-91

Naoi M, Maruyama W, Akao Y, Zhang J, Parvez H. 2000. Apoptosis induced by an endogenous neurotoxin, $\mathrm{N}$-methyl(R)salsolinol, in dopamine neurons. Toxicology 153:123-41

Naoi M, Maruyama W, Dostert P, Hashizume Y, Nakahara D, et al. 1996a. Dopaminederived endogenous 1(R),2(N)-dimethyl-6,7-dihydroxy- 1,2,3,4-tetrahydroisoquinoline, $\mathrm{N}$-methyl-(R)-salsolinol, induced parkinsonism in rat: biochemical, pathological and behavioral studies. Brain Res 709:285-95

Naoi M, Maruyama W, Dostert P, Kohda K, Kaiya T. 1996b. A novel enzyme enantioselectively synthesizes (R)salsolinol, a precursor of a dopaminergic neurotoxin, $\mathrm{N}$ methyl(R)salsolinol. Neurosci Lett 212:183-6

Naoi M, Maruyama W, Dostert P, Nakahara D, Takahashi T, Nagatsu T. 1995. Metabolic bioactivation of endogenous isoquinolines as dopaminergic neurotoxins to elicit

Parkinson's disease. In Alzheimer's and Parkinson's Diseases. ed. I Hanin, pp. 553-9. New York: Plenum Press

Naoi M, Maruyama W, Kasamatsu T, Dostert P. 1998a. Oxidation of Nmethyl(R)salsolinol: involvement to neurotoxicity and neuroprotection by endogenous catechol isoquinolines. J Neural Transm Suppl 52:125-38 
Naoi M, Maruyama W, Matsubara K, Hashizume Y. 1997. A neutral N-methyltransferase activity in the striatum determines the level of an endogenous MPP+-like neurotoxin, 1,2dimethyl-6,7-dihydroxyisoquinolinium ion, in the substantia nigra of human brains.

Neurosci Lett 235:81-4

Naoi M, Maruyama W, Nagy GM. 2004. Dopamine-derived salsolinol derivatives as endogenous monoamine oxidase inhibitors: occurrence, metabolism and function in human brains. Neurotoxicology 25:193-204

Naoi M, Maruyama W, Nakao N, Ibi T, Sahashi K, Benedetti MS. 1998b. (R)salsolinol Nmethyltransferase activity increases in parkinsonian lymphocytes. Ann Neurol 43:212-6

Nappi AJ, Vass E, Collins MA. 1999. Contrasting effects of catecholic and O-methylated tetrahydroisoquinolines on hydroxyl radical production. Biochim Biophys Acta 1434:6473

Narhi L, Wood SJ, Steavenson S, Jiang Y, Wu GM, et al. 1999. Both familial Parkinson's disease mutations accelerate alpha-synuclein aggregation. J Biol Chem 274:9843-6

Nelson MC, Lytle LA. 2009. Development and evaluation of a brief screener to estimate fast-food and beverage consumption among adolescents. J Am Diet Assoc 109:730-4

Nijima K, Araki M, Ogawa M, Suzuki K, Mizuno Y, et al. 1991. N-methylisoquinolinium ion (NMIQ+) destroys cultured mesencephalic dopamine neurons. Biogenic Amines 8:61-7

Nishi K, Mochizuki H, Furukawa Y, Mizuno Y, Yoshida M. 1994. Neurotoxic effects of 1methyl-4-phenylpyridinium (MPP+) and tetrahydroisoquinoline derivatives on dopaminergic neurons in ventral mesencephalic-striatal co-culture. Neurodegeneration 3:33-42

Niwa T, Takeda N, Kaneda N, Hashizume Y, Nagatsu T. 1987. Presence of tetrahydroisoquinoline and 2-methyl-tetrahydroquinoline in parkinsonian and normal human brains. Biochem Biophys Res Commun 144:1084-9

Niwa T, Takeda N, Yoshizumi H, Tatematsu A, Yoshida M, et al. 1991. Presence of 2methyl-6,7-dihydroxy-1,2,3,4-tetrahydroisoquinoline and 1,2-dimethyl-6,7-dihydroxy1,2,3,4-tetrahydroisoquinoline, novel endogenous amines, in parkinsonian and normal human brains. Biochem Biophys Res Commun 177:603-9

Niwa T, Takeda N, Yoshizumi H, Tatematsu A, Yoshida M, et al. 1993. Presence of tetrahydroisoquinoline-related compounds, possible MPTP-like neurotoxins, in Parkinsonian brain In Advances in Neurology ed. H Narabayashi, T Nagatsu, $\mathrm{N}$ Yanagisawa, Y Mizuno, pp. 234-7. New York: Raven Press, Ltd.

Niwa T, Yoshizumi H, Tatematsu A, Matsuura S, Nagatsu T. 1989. Presence of tetrahydroisoquinoline, a parkinsonism-related compound, in foods. J Chromatogr 493:347-52

Norris EH, Giasson BI, Lee VM. 2004. Alpha-synuclein: normal function and role in neurodegenerative diseases. Curr Top Dev Biol 60:17-54 
Nutt JG. 1990. Levodopa-induced dyskinesia: review, observations, and speculations. Neurology 40:340-5

Nutt JG, Carter JH. 1984. Sensory symptoms in parkinsonism related to central dopaminergic function. Lancet 2:456-7

Nutt JG, Marsden CD, Thompson PD. 1993. Human walking and higher-level gait disorders, particularly in the elderly. Neurology 43:268-79

Ogawa M, Araki M, Nagatsu I, Nagatsu T, Yoshida M. 1989. The effect of 1,2,3,4tetrahydroisoquinoline (TIQ) on mesencephalic dopaminergic neurons in C57BL/6J

mice: immunohistochemical studies-tyrosine hydroxylase. Biogenic Amines 26:2061-5

Ohta S, Kohno K, Makino Y, Tachikawa O, Hirobe M. 1987. Tetrahydroisoquinoline and 1-methyl-tetrahydroisoquinoline are present in the human brain: relation to Parkinson's disease. Biomedical Research 8:453-6

Ohta S, Tachikawa O, Makino Y, Tasaki Y, Hirobe M. 1990. Metabolism and brain accumulation of tetrahydroisoquinoline (TIQ) a possible parkinsonism inducing substance, in an animal model of a poor debrisoquine metabolizer. Life Sci 46:599-605

Ohye C, Saito U, Fukamachi A, Narabayashi H. 1974. An analysis of the spontaneous rhythmic and non-rhythmic burst discharges in the human thalamus. $J$ Neurol Sci 22:245-59

Okazaki H, Lipkin LE, Aronson SM. 1961. Diffuse intracytoplasmic ganglionic inclusions (Lewy type) associated with progressive dementia and quadriparesis in flexion. $J$ Neuropathol Exp Neurol 20:237-44

Origitano T, Hannigan J, Collins MA. 1981. Rat brain salsolinol and blood-brain barrier. Brain Res 224:446-51

Origitano TC, Collins MA. 1980. Confirmation of an unexpected brain O-methylation pattern for the dopamine-derived tetrahydroisoquinoline, salsolinol. Life Sci 26:2061-5

Ostrerova-Golts N, Petrucelli L, Hardy J, Lee JM, Farer M, Wolozin B. 2000. The A53T alpha-synuclein mutation increases iron-dependent aggregation and toxicity. $J$ Neurosci 20:6048-54

Ouyang M, Shen X. 2006. Critical role of ASK1 in the 6-hydroxydopamine-induced apoptosis in human neuroblastoma SH-SY5Y cells. J Neurochem 97:234-44

Pacifici GM, Temellini A, Castiglioni M, D'Alessandro C, Ducci A, Giuliani L. 1994. Interindividual variability of the human hepatic sulphotransferases. Chem Biol Interact 92:219-31

Paganini-Hill A. 2001. Risk factors for Parkinson's disease: the leisure world cohort study. Neuroepidemiology 20:118-24

Paisan-Ruiz C, Jain S, Evans EW, Gilks WP, Simon J, et al. 2004. Cloning of the gene containing mutations that cause PARK8-linked Parkinson's disease. Neuron 44:595-600 
Palacino JJ, Sagi D, Goldberg MS, Krauss S, Motz C, et al. 2004. Mitochondrial dysfunction and oxidative damage in parkin-deficient mice. J Biol Chem 279:18614-22

Parker WD, Jr., Boyson SJ, Parks JK. 1989. Abnormalities of the electron transport chain in idiopathic Parkinson's disease. Ann Neurol 26:719-23

Parkinson J. 2002. An essay on the shaking palsy. 1817. J Neuropsychiatry Clin Neurosci 14:223-36; discussion 2

Parrado J, Absi E, Ayala A, Castano A, Cano J, Machado A. 2000. The endogenous amine 1-methyl-1,2,3,4- tetrahydroisoquinoline prevents the inhibition of complex I of the respiratory chain produced by MPP(+). J Neurochem 75:65-71

Parsons RB, Smith SW, Waring RH, Williams AC, Ramsden DB. 2003. High expression of nicotinamide $\mathrm{N}$-methyltransferase in patients with idiopathic Parkinson's disease. Neurosci Lett 342:13-6

Perry TL, Jones K, Hansen S. 1988. Tetrahydroisoquinoline lacks dopaminergic nigrostriatal neurotoxicity in mice. Neurosci Lett 85:101-4

Perry TL, Jones K, Hansen S, Wall RA. 1987. 4-phenylpyridine and three other analogues of 1-methyl-4-phenyl-1,2,3,6-tetrahydropyridine lack dopaminergic nigrostriatal neurotoxicity in mice and marmosets. Neurosci Lett 75:65-70

Petrucelli L, O'Farrell C, Lockhart PJ, Baptista M, Kehoe K, et al. 2002. Parkin protects against the toxicity associated with mutant alpha-synuclein: proteasome dysfunction selectively affects catecholaminergic neurons. Neuron 36:1007-19

Pillon B, Dubois B, Agid Y. 1996. Testing cognition may contribute to the diagnosis of movement disorders. Neurology 46:329-34

Pollanen MS, Dickson DW, Bergeron C. 1993. Pathology and biology of the Lewy body. $\checkmark$ Neuropathol Exp Neurol 52:183-91

Polymeropoulos MH, Higgins JJ, Golbe LI, Johnson WG, Ide SE, et al. 1996. Mapping of a gene for Parkinson's disease to chromosome 4q21-q23. Science 274:1197-9

Polymeropoulos MH, Lavedan C, Leroy E, Ide SE, Dehejia A, et al. 1997. Mutation in the alpha-synuclein gene identified in families with Parkinson's disease. Science 276:2045-7

Poole AC, Thomas RE, Andrews LA, McBride HM, Whitworth AJ, Pallanck LJ. 2008. The PINK1/Parkin pathway regulates mitochondrial morphology. Proc Natl Acad Sci U S A 105:1638-43

Psarra AM, Sekeris CE. 2008. Nuclear receptors and other nuclear transcription factors in mitochondria: regulatory molecules in a new environment. Biochim Biophys Acta 1783:1-11

Puigserver P, Rhee J, Donovan J, Walkey CJ, Yoon JC, et al. 2003. Insulin-regulated hepatic gluconeogenesis through FOXO1-PGC-1alpha interaction. Nature 423:550-5 
Qualman SJ, Haupt HM, Yang P, Hamilton SR. 1984. Esophageal Lewy bodies associated with ganglion cell loss in achalasia. Similarity to Parkinson's disease. Gastroenterology 87:848-56

Quinn NP, Koller WC, Lang AE, Marsden CD. 1986. Painful Parkinson's disease. Lancet $1: 1366-9$

Rajput AH. 1992. Frequency and cause of Parkinson's disease. Can J Neurol Sci 19:103-7

Rajput AH, Birdi S. 1997. Epidemiology of Parkinson's disease. Parkinsonism Relat Disord 3:175-86

Rajput AH, Offord KP, Beard CM, Kurland LT. 1984. Epidemiology of parkinsonism: incidence, classification, and mortality. Ann Neurol 16:278-82

Rajput AH, Pahwa R, Pahwa P, Rajput A. 1993. Prognostic significance of the onset mode in parkinsonism. Neurology 43:829-30

Rajput AH, Rozdilsky B, Rajput A. 1991. Accuracy of clinical diagnosis in parkinsonism-a prospective study. Can J Neurol Sci 18:275-8

Rajput AH, Uitti RJ, Stern W, Laverty W. 1986. Early onset Parkinson's disease in Saskatchewan--environmental considerations for etiology. Can J Neurol Sci 13:312-6

Reddehase S, Grumbt B, Neupert W, Hell K. 2009. The disulfide relay system of mitochondria is required for the biogenesis of mitochondrial Ccs1 and Sod1. $J$ Mol Biol 385:331-8

Reijnders JS, Ehrt U, Weber WE, Aarsland D, Leentjens AF. 2008. A systematic review of prevalence studies of depression in Parkinson's disease. Mov Disord 23:183-9; quiz 313

Richter D. 1940. The inactivation of adrenaline in vivo in man. J Physiol 98:361-74

Rideout HJ, Lang-Rollin I, Stefanis L. 2004. Involvement of macroautophagy in the dissolution of neuronal inclusions. Int J Biochem Cell Biol 36:2551-62

Rideout HJ, Larsen KE, Sulzer D, Stefanis L. 2001. Proteasomal inhibition leads to formation of ubiquitin/alpha-synuclein-immunoreactive inclusions in PC12 cells. $J$ Neurochem 78:899-908

Riggin RM, Kissinger PT. 1976. Letter: Identification of salsolinol as a phenolic component in powdered cocoa and cocoa-based products. J Agric Food Chem 24:900

Riggin RM, McCarthy MJ, Kissinger PT. 1976. Identification of salsolinol as a major dopamine metabolite in the banana. J Agric Food Chem 24:189-91

Rommelspacher H, Susilo R. 1985. Tetrahydroisoquinolines and beta-carbolines: putative natural substances in plants and mammals. Prog Drug Res 29:415-59 
Rosati G, Granieri E, Pinna L, Aiello I, Tola R, et al. 1980. The risk of Parkinson disease in Mediterranean people. Neurology 30:250-5

Ross CA, Pickart CM. 2004. The ubiquitin-proteasome pathway in Parkinson's disease and other neurodegenerative diseases. Trends Cell Biol 14:703-11

Roy S, Wolman L. 1969. Ultrastructural observations in Parkinsonism. J Pathol 99:39-44

Sandler M, Carter SB, Hunter KR, Stern GM. 1973. Tetrahydroisoquinoline alkaloids: in vivo metabolites of L-dopa in man. Nature 241:439-43

Sasaoka T, Kaneda N, Niwa T, Hashizume Y, Nagatsu T. 1988. Analysis of salsolinol in human brain using high-performance liquid chromatography with electrochemical detection. J Chromatogr 428:152-5

Sato C, Morgan A, Lang AE, Salehi-Rad S, Kawarai T, et al. 2005. Analysis of the glucocerebrosidase gene in Parkinson's disease. Mov Disord 20:367-70

Schapira AH. 2001. Causes of neuronal death in Parkinson's disease. Adv Neurol 86:155-62

Schapira AH, Mann VM, Cooper JM, Dexter D, Daniel SE, et al. 1990. Anatomic and disease specificity of NADH CoQ1 reductase (complex I) deficiency in Parkinson's disease. J Neurochem 55:2142-5

Schmidt ML, Murray J, Lee VM, Hill WD, Wertkin A, Trojanowski JQ. 1991. Epitope map of neurofilament protein domains in cortical and peripheral nervous system Lewy bodies. Am J Pathol 139:53-65

Schoenberg BS. 1987. Environmental risk factors for Parkinson's disease: the epidemiologic evidence. Can J Neurol Sci 14:407-13

Schoenberg BS, Anderson DW, Haerer AF. 1985. Prevalence of Parkinson's disease in the biracial population of Copiah County, Mississippi. Neurology 35:841-5

Schoenberg BS, Osuntokun BO, Adeuja AO, Bademosi O, Nottidge V, et al. 1988. Comparison of the prevalence of Parkinson's disease in black populations in the rural United States and in rural Nigeria: door-to-door community studies. Neurology 38:645-6

Scholz J, Bamberg H, Moser A. 1997. N-methyl-norsalsolinol, an endogenous neurotoxin, inhibits tyrosine hydroxylase activity in the rat brain nucleus accumbens in vitro. Neurochem Int 31:845-9

Scholz J, Klingemann I, Moser A. 2004. Increased systemic levels of norsalsolinol derivatives are induced by levodopa treatment and do not represent biological markers of Parkinson's disease. J Neurol Neurosurg Psychiatry 75:634-6

Schöpf C, Bayerle H. 1934. Zur frage der biogenese der isochinolin-alkaloide. Ann Chem 513:190-202 
Schulman S, Fernando Q. 1968. Excited state prototropic equilibria of some quinolonols. Tetrahedron 24:1777-83

Seidler A, Hellenbrand W, Robra BP, Vieregge P, Nischan P, et al. 1996. Possible environmental, occupational, and other etiologic factors for Parkinson's disease: a casecontrol study in Germany. Neurology 46:1275-84

Selby G. 1984. The Graeme Robertson memorial lecture, 1983. The long-term prognosis of Parkinson's disease. Clin Exp Neurol 20:1-25

Semchuk KM, Love EJ, Lee RG. 1991. Parkinson's disease and exposure to rural environmental factors: a population based case-control study. Can J Neurol Sci 18:27986

Semchuk KM, Love EJ, Lee RG. 1992. Parkinson's disease and exposure to agricultural work and pesticide chemicals. Neurology 42:1328-35

Shavali S, Ebadi M. 2003. 1-Benzyl-1,2,3,4-tetrahydroisoquinoline (1BnTIQ), an endogenous neurotoxin, induces dopaminergic cell death through apoptosis. Neurotoxicology 24:417-24

Shavali S, Ren J, Ebadi M. 2003. Insulin-like growth factor-1 protects human dopaminergic SH-SY5Y cells from salsolinol-induced toxicity. Neurosci Lett 340:79-82

Sherer TB, Betarbet R, Kim JH, Greenamyre JT. 2003. Selective microglial activation in the rat rotenone model of Parkinson's disease. Neurosci Lett 341:87-90

Shimura H, Hattori N, Kubo S, Mizuno Y, Asakawa S, et al. 2000. Familial Parkinson disease gene product, parkin, is a ubiquitin-protein ligase. Nat Genet 25:302-5

Shulman LM. 2007. Gender differences in Parkinson's disease. Gend Med 4:8-18

Shults CW. 2004. Mitochondrial dysfunction and possible treatments in Parkinson's disease--a review. Mitochondrion 4:641-8

Siddhuraju P, Becker K, Makkar HP. 2000. Studies on the nutritional composition and antinutritional factors of three different germplasm seed materials of an under-utilized tropical legume, Mucuna pruriens var. utilis. J Agric Food Chem 48:6048-60

Sidransky E, Nalls MA, Aasly JO, Aharon-Peretz J, Annesi G, et al. 2009. Multicenter analysis of glucocerebrosidase mutations in Parkinson's disease. N Engl J Med 361:1651-61

Silvestri L, Caputo V, Bellacchio E, Atorino L, Dallapiccola B, et al. 2005. Mitochondrial import and enzymatic activity of PINK1 mutants associated to recessive parkinsonism. Hum Mol Genet 14:3477-92

Singh M, Khanna VK, Shukla R, Parmar D. 2010. Association of polymorphism in cytochrome P450 2D6 and N-acetyltransferase-2 with Parkinson's disease. Dis Markers 28:87-93 
Singleton AB, Farrer M, Johnson J, Singleton A, Hague S, et al. 2003. alpha-Synuclein locus triplication causes Parkinson's disease. Science 302:841

Sjoquist B, Eriksson A, Winblad B. 1982a. Brain salsolinol levels in alcoholism. Lancet 1:675-6

Sjoquist B, Eriksson A, Winblad B. 1982b. Salsolinol and catecholamines in human brain and their relation to alcoholism. Prog Clin Biol Res 90:57-67

Skulachev VP. 1998. Cytochrome $\mathrm{c}$ in the apoptotic and antioxidant cascades. FEBS Lett 423:275-80

Smeyne RJ, Jackson-Lewis V. 2005. The MPTP model of Parkinson's disease. Brain Res Mol Brain Res 134:57-66

Snider SR, Fahn S, Isgreen WP, Cote LJ. 1976. Primary sensory symptoms in parkinsonism. Neurology 26:423-9

Snyder H, Wolozin B. 2004. Pathological proteins in Parkinson's disease: focus on the proteasome. J Mol Neurosci 24:425-42

Song Y, Xu J, Hamme A, Liu YM. 2006. Capillary liquid chromatography-tandem mass spectrometry of tetrahydroisoquinoline derived neurotoxins: a study on the blood-brain barrier of rat brain. J Chromatogr A 1103:229-34

Soto-Otero R, Mendez-Alvarez E, Sanchez-Sellero I, Cruz-Landeira A, Lopez-Rivadulla Lamas M. 2001. Reduction of rat brain levels of the endogenous dopaminergic proneurotoxins 1,2,3,4-tetrahydroisoquinoline and 1,2,3,4-tetrahydro-beta-carboline by cigarette smoke. Neurosci Lett 298:187-90

Soto-Otero R, Riguera-Vega R, Mendez-Alvarez E, Sanchez-Sellero I, Lopez-Rivadulla Lamas M. 1996. Interaction of 1,2,3,4-tetrahydroisoquinoline with some components of cigarette smoke: potential implications for Parkinson's disease. Biochem Biophys Res Commun 222:607-11

Spillantini MG, Crowther RA, Jakes R, Hasegawa M, Goedert M. 1998. alpha-Synuclein in filamentous inclusions of Lewy bodies from Parkinson's disease and dementia with lewy bodies. Proc Natl Acad Sci U S A 95:6469-73

Spillantini MG, Schmidt ML, Lee VM, Trojanowski JQ, Jakes R, Goedert M. 1997. Alphasynuclein in Lewy bodies. Nature 388:839-40

Stefanis L, Larsen KE, Rideout HJ, Sulzer D, Greene LA. 2001. Expression of A53T mutant but not wild-type alpha-synuclein in PC12 cells induces alterations of the ubiquitin-dependent degradation system, loss of dopamine release, and autophagic cell death. J Neurosci 21:9549-60

Stefanova N, Klimaschewski L, Poewe W, Wenning GK, Reindl M. 2001. Glial cell death induced by overexpression of alpha-synuclein. J Neurosci Res 65:432-8 
Storch A, Kaftan A, Burkhardt K, Schwarz J. 2000. 1-Methyl-6,7-dihydroxy-1,2,3,4tetrahydroisoquinoline (salsolinol) is toxic to dopaminergic neuroblastoma SH-SY5Y cells via impairment of cellular energy metabolism. Brain Res 855:67-75

Storch A, Ott S, Hwang YI, Ortmann R, Hein A, et al. 2002. Selective dopaminergic neurotoxicity of isoquinoline derivatives related to Parkinson's disease: studies using heterologous expression systems of the dopamine transporter. Biochem Pharmacol 63:909-20

Svenson LW. 1991. Regional disparities in the annual prevalence rates of Parkinson's disease in Canada. Neuroepidemiology 10:205-10

Svenson LW, Platt GH, Woodhead SE. 1993. Geographic variations in the prevalence rates of Parkinson's disease in Alberta. Can J Neurol Sci 20:307-11

Taha JM, Favre J, Baumann TK, Burchiel KJ. 1996. Characteristics and somatotopic organization of kinesthetic cells in the globus pallidus of patients with Parkinson's disease. J Neurosurg 85:1005-12

Takahashi T, Deng Y, Maruyama W, Dostert P, Kawai M, Naoi M. 1994. Uptake of a neurotoxin-candidate, (R)-1,2-dimethyl-6,7-dihydroxy-1,2,3,4-tetrahydroisoquinoline into human dopaminergic neuroblastoma SH-SY5Y cells by dopamine transport system. $J$ Neural Transm Gen Sect 98:107-18

Takahashi T, Maruyama W, Deng Y, Dostert P, Nakahara D, et al. 1997. Cytotoxicity of endogenous isoquinolines to human dopaminergic neuroblastoma SH-SY5Y cells. $J$ Neural Transm 104:59-66

Takeda A, Mallory M, Sundsmo M, Honer W, Hansen L, Masliah E. 1998. Abnormal accumulation of NACP/alpha-synuclein in neurodegenerative disorders. Am J Pathol 152:367-72

Tanaka Y, Engelender S, Igarashi S, Rao RK, Wanner T, et al. 2001. Inducible expression of mutant alpha-synuclein decreases proteasome activity and increases sensitivity to mitochondria-dependent apoptosis. Hum Mol Genet 10:919-26

Tanner CM, Chen B, Wang W, Peng M, Liu Z, et al. 1989. Environmental factors and Parkinson's disease: a case-control study in China. Neurology 39:660-4

Tanner CM, Chen B, Wang WZ, Peng ML, Liu ZL, et al. 1987. Environmental factors in the etiology of Parkinson's disease. Can J Neurol Sci 14:419-23

Tanner CM, Ottman R, Goldman SM, Ellenberg J, Chan P, et al. 1999. Parkinson disease in twins: an etiologic study. JAMA 281:341-6

Tasaki Y, Makino Y, Ohta S, Hirobe M. 1991. 1-Methyl-1,2,3,4-tetrahydroisoquinoline, decreasing in 1-methyl-4-phenyl-1,2,3,6-tetrahydropyridine-treated mouse, prevents parkinsonism-like behavior abnormalities. J Neurochem 57:1940-3 
Tatton WG, Bedingham W, Verrier MC, Blair RD. 1984. Characteristic alterations in responses to imposed wrist displacements in parkinsonian rigidity and dystonia musculorum deformans. Can J Neurol Sci 11:281-7

Taylor KS, Cook JA, Counsell CE. 2007. Heterogeneity in male to female risk for Parkinson's disease. J Neurol Neurosurg Psychiatry 78:905-6

Teismann P, Schulz JB. 2004. Cellular pathology of Parkinson's disease: astrocytes, microglia and inflammation. Cell Tissue Res 318:149-61

Teitel S, O'Brien J, Brossi A. 1972. Alkaloids in mammalian tissues. 2. Synthesis of (+)and (-)-1-substituted-6,7-dihydroxy-1,2,3,4-tetrahydroisoquinolines. J Med Chem 15:8456

Tennyson VM, Cohen G, Mytilineou C, Heikkila R. 1973. 6,7Dihydroxytetrahydroisoquinoline: electron microscopic evidence for uptake into the amine-binding vesicles in sympathetic nerves of rat iris and pineal gland. Brain Res $51: 161-9$

Thull U, Kneubuhler S, Gaillard P, Carrupt PA, Testa B, et al. 1995. Inhibition of monoamine oxidase by isoquinoline derivatives. Qualitative and 3D-quantitative structure-activity relationships. Biochem Pharmacol 50:869-77

Thumen A, Behnecke A, Qadri F, Bauml E, Behnecke CA, Moser A. 2002. N-methylnorsalsolinol, a putative dopaminergic neurotoxin, passes through the blood-brain barrier in vivo. Neuroreport 13:25-8

Tipton KF, Singer TP. 1993. Advances in our understanding of the mechanisms of the neurotoxicity of MPTP and related compounds. J Neurochem 61:1191-206

Tofaris GK, Layfield R, Spillantini MG. 2001. alpha-synuclein metabolism and aggregation is linked to ubiquitin-independent degradation by the proteasome. FEBS Lett 509:22-6

Toth BE, Homicsko K, Radnai B, Maruyama W, DeMaria JE, et al. 2001. Salsolinol is a putative endogenous neuro-intermediate lobe prolactin-releasing factor. $J$ Neuroendocrinol 13:1042-50

Twelves D, Perkins KS, Counsell C. 2003. Systematic review of incidence studies of Parkinson's disease. Mov Disord 18:19-31

Ueda K, Fukushima H, Masliah E, Xia Y, Iwai A, et al. 1993. Molecular cloning of cDNA encoding an unrecognized component of amyloid in Alzheimer disease. Proc Natl Acad Sci U S A 90:11282-6

Uitti RJ, Rajput AH, Ashenhurst EM, Rozdilsky B. 1985. Cyanide-induced parkinsonism: a clinicopathologic report. Neurology 35:921-5

Uversky VN, Fink AL. 2002. Amino acid determinants of alpha-synuclein aggregation: putting together pieces of the puzzle. FEBS Lett 522:9-13 
Valente EM, Abou-Sleiman PM, Caputo V, Muqit MM, Harvey K, et al. 2004. Hereditary early-onset Parkinson's disease caused by mutations in PINK1. Science 304:1158-60

Valente EM, Bentivoglio AR, Dixon PH, Ferraris A, lalongo T, et al. 2001. Localization of a novel locus for autosomal recessive early-onset parkinsonism, PARK6, on human chromosome 1p35-p36. Am J Hum Genet 68:895-900

Valverde GDAD, Madureira de Oliveria D, Barreto G, Bertolino LA, Saraceno E, et al. 2008. Effects of the extract of Anemopaegma mirandum (Catuaba) on Rotenoneinduced apoptosis in human neuroblastomas SH-SY5Y cells. Brain Res 1198:188-96

Van Den Eeden SK, Tanner CM, Bernstein AL, Fross RD, Leimpeter A, et al. 2003. Incidence of Parkinson's disease: variation by age, gender, and race/ethnicity. Am J Epidemiol 157:1015-22

van Duijn CM, Dekker MC, Bonifati V, Galjaard RJ, Houwing-Duistermaat JJ, et al. 2001. Park7, a novel locus for autosomal recessive early-onset parkinsonism, on chromosome 1p36. Am J Hum Genet 69:629-34

Vanacore N, Bonifati V, Bellatreccia A, Edito F, Meco G. 1992. Mortality rates for Parkinson's disease and parkinsonism in Italy (1969-1987). Neuroepidemiology 11:65-73

Vincken WG, Gauthier SG, Dollfuss RE, Hanson RE, Darauay CM, Cosio MG. 1984. Involvement of upper-airway muscles in extrapyramidal disorders. A cause of airflow limitation. N Engl J Med 311:438-42

Virbasius CA, Virbasius JV, Scarpulla RC. 1993. NRF-1, an activator involved in nuclearmitochondrial interactions, utilizes a new DNA-binding domain conserved in a family of developmental regulators. Genes Dev 7:2431-45

Virbasius JV, Scarpulla RC. 1994. Activation of the human mitochondrial transcription factor A gene by nuclear respiratory factors: a potential regulatory link between nuclear and mitochondrial gene expression in organelle biogenesis. Proc Natl Acad Sci U S A 91:1309-13

von Campenhausen S, Bornschein B, Wick R, Botzel K, Sampaio C, et al. 2005. Prevalence and incidence of Parkinson's disease in Europe. Eur Neuropsychopharmacol 15:473-90

Wakabayashi K, Matsumoto K, Takayama K, Yoshimoto M, Takahashi H. 1997. NACP, a presynaptic protein, immunoreactivity in Lewy bodies in Parkinson's disease. Neurosci Lett 239:45-8

Wallace DC. 2005. A mitochondrial paradigm of metabolic and degenerative diseases, aging, and cancer: a dawn for evolutionary medicine. Annu Rev Genet 39:359-407

Wang W, Ameno K, Jamal M, Kumihashi M, Uekita I, et al. 2007. Effect of direct infusion of acetaldehyde on dopamine and dopamine-derived salsolinol in the striatum of freemoving rats using a reverse microdialysis technique. Arch Toxicol 81:121-6 
Wanpen S, Govitrapong P, Shavali S, Sangchot P, Ebadi M. 2004. Salsolinol, a dopamine-derived tetrahydroisoquinoline, induces cell death by causing oxidative stress in dopaminergic SH-SY5Y cells, and the said effect is attenuated by metallothionein. Brain Res 1005:67-76

Ward AA, Jr., Mc CW, Magoun HW. 1948. Production of an alternating tremor at rest in monkeys. J Neurophysiol 11:317-30

Webb JL, Ravikumar B, Atkins J, Skepper JN, Rubinsztein DC. 2003. Alpha-Synuclein is degraded by both autophagy and the proteasome. J Biol Chem 278:25009-13

White RJ, Reynolds IJ. 1996. Mitochondrial depolarization in glutamate-stimulated neurons: an early signal specific to excitotoxin exposure. J Neurosci 16:5688-97

Whitehouse PJ. 1987. Clinical and neurochemical consequences of neuronal loss in the nucleus basalis of Meynert in Parkinson's disease and Alzheimer's disease. Adv Neurol 45:393-7

Willets JM, Lambert DG, Lunec J, Griffiths HR. 1995. Studies on the neurotoxicity of 6,7dihydroxy-1-methyl-1,2,3,4-tetrahydroisoquinoline (salsolinol) in SH-SY5Y cells. Eur J Pharmacol 293:319-26

Yamada Y. 1971. Effects of salsolinol on rat brain and liver monoamine oxidase. Jpn J Pharmacol 21:833-5

Yamakawa T, Kotake Y, Fujitani M, Shintani H, Makino Y, Ohta S. 1999. Regional distribution of parkinsonism-preventing endogenous tetrahydroisoquinoline derivatives and an endogenous parkinsonism-preventing substance-synthesizing enzyme in monkey brain. Neurosci Lett 276:68-70

Yi H, Akao Y, Maruyama W, Chen K, Shih J, Naoi M. 2006a. Type A monoamine oxidase is the target of an endogenous dopaminergic neurotoxin, $\mathrm{N}$-methyl(R)salsolinol, leading to apoptosis in SH-SY5Y cells. J Neurochem 96:541-9

Yi H, Maruyama W, Akao Y, Takahashi T, Iwasa K, et al. 2006b. N-Propargylamine protects SH-SY5Y cells from apoptosis induced by an endogenous neurotoxin, $\mathrm{N}$ methyl(R)salsolinol, through stabilization of mitochondrial membrane and induction of anti-apoptotic Bcl-2. J Neural Transm 113:21-32

Yoshida M, Ogawa M, Suzuki K, Nagatsu T. 1993. Parkinsonism produced by tetrahydroisoquinoline (TIQ) or the analogues. Adv Neurol 60:207-11

Youle RJ, Karbowski M. 2005. Mitochondrial fission in apoptosis. Nat Rev Mol Cell Biol 6:657-63

Young WF, Jr., Okazaki H, Laws ER, Jr., Weinshilboum RM. 1984. Human brain phenol sulfotransferase: biochemical properties and regional localization. J Neurochem 43:70615

Yuan J, Yankner BA. 2000. Apoptosis in the nervous system. Nature 407:802-9 
Yuan SS, Chang HL, Chen HW, Yeh YT, Kao YH, et al. 2003. Annonacin, a monotetrahydrofuran acetogenin, arrests cancer cells at the $\mathrm{G} 1$ phase and causes cytotoxicity in a Bax- and caspase-3-related pathway. Life Sci 72:2853-61 
APPENDIX A. THERMAL STABILITY AND MOLECULAR PROPERTIES OF TETRAHYDROISOQUINOLINES 
Table A-1. Limits of detection and quantification.

\begin{tabular}{lccc}
\hline Compound & LOD* $^{*}(\mathbf{n g} / \mathbf{m l})$ & LOQ $^{*}(\mathbf{n g} / \mathbf{m l})$ & LOD / LOQ Ratio \\
\hline TIQ & 0.0288 & 0.30 & 10.417 \\
1-Methyl-TIQ & 0.0254 & 0.30 & 11.811 \\
N-Methyl-TIQ & 0.0294 & 0.30 & 10.204 \\
1-Benzyl-TIQ & 0.0219 & 0.30 & 13.699 \\
(R)SAL & 0.0629 & 0.70 & 11.129 \\
(S)SAL & 0.0634 & 0.70 & 11.041 \\
N-Methyl-(R)SAL & 0.0643 & 0.70 & 10.886 \\
N-Methyl-(S)SAL & 0.0651 & 0.70 & 10.753 \\
NorSAL & 0.0549 & 0.70 & 12.750 \\
Dopamine & 0.0365 & 0.70 & 19.178 \\
DOPAC & 0.0392 & 0.70 & 17.857 \\
HVA & 0.0406 & 0.70 & 17.241 \\
\hline
\end{tabular}

* LOD = limit of detection.

* $L O Q=$ limit of quantification. 


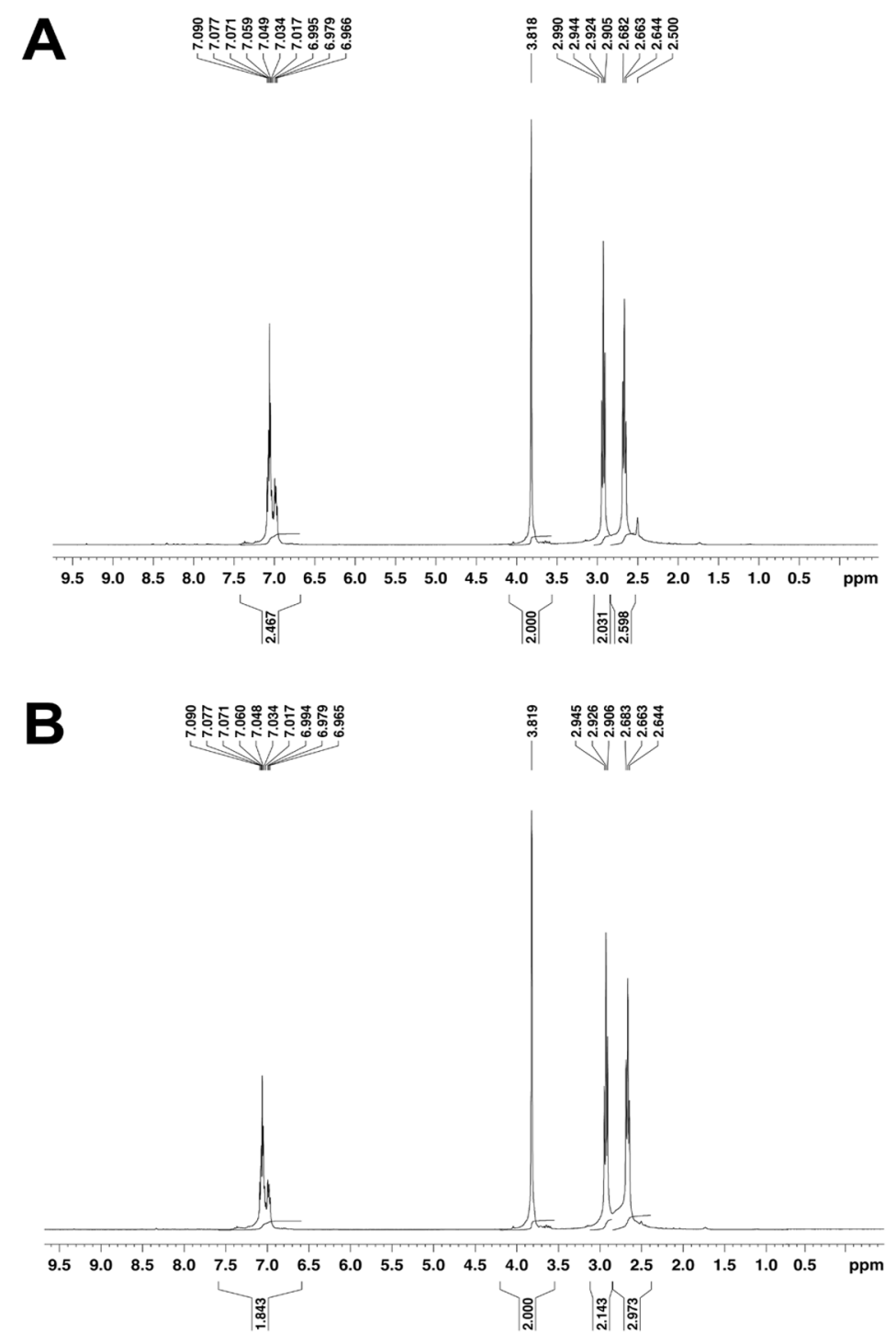

Figure A-1. ${ }^{1} \mathrm{H}$ NMR spectra of TIQ.

Comparison of chemical shift (lower bracketed values) and peak integration (upper values) at baseline (A) and after 28 days of heat exposure at $60^{\circ} \mathrm{C}(\mathrm{B})$ reveals no significant structural changes after thermal challenge. 

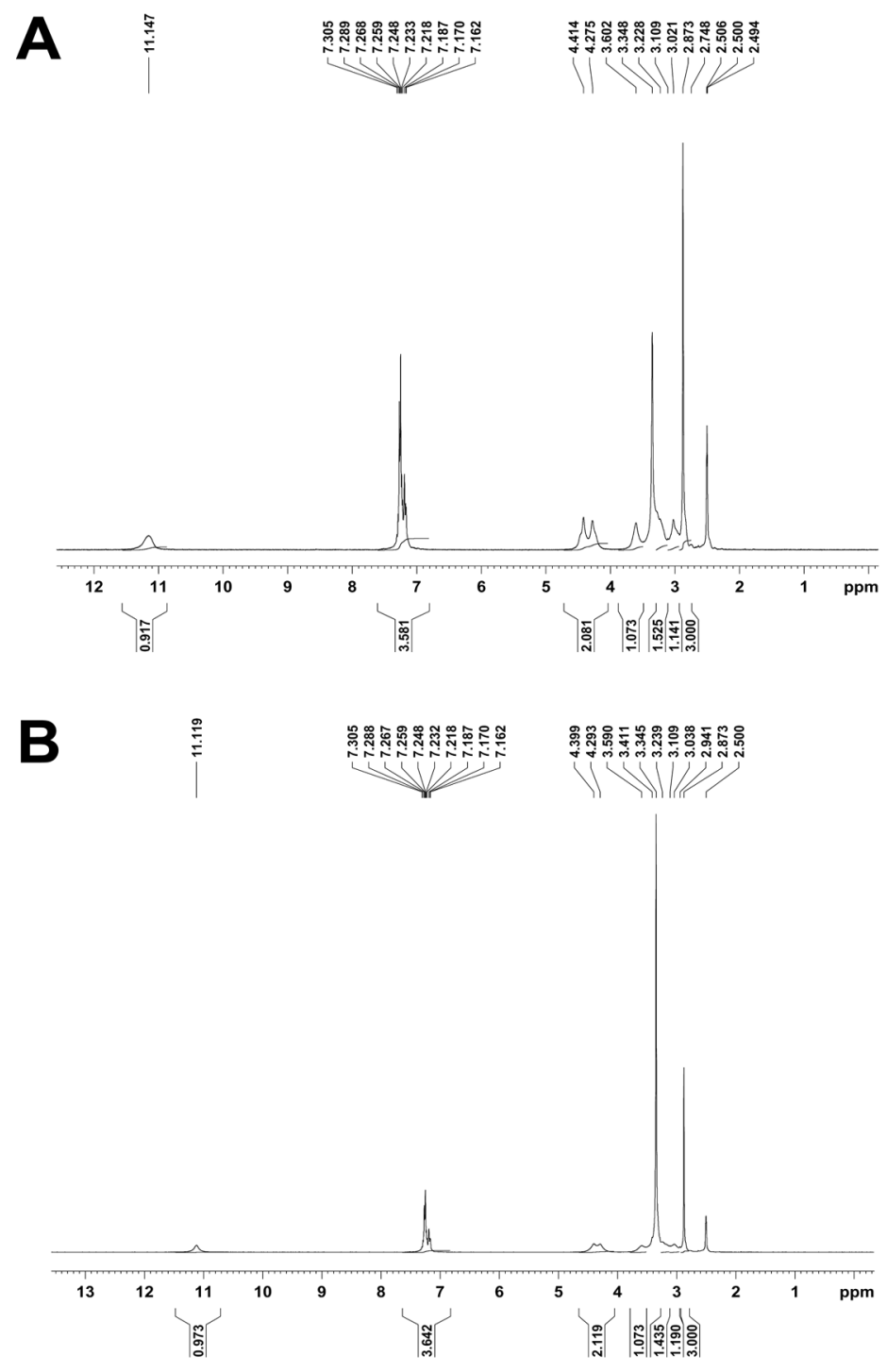

Figure A-2. ${ }^{1} \mathrm{H}$ NMR spectra of $\mathbf{N}$-methyl-TIQ.

Comparison of chemical shift (lower bracketed values) and peak integration (upper values) at baseline (A) and after 28 days of heat exposure at $60^{\circ} \mathrm{C}(\mathrm{B})$ reveals no significant structural changes after thermal challenge. 

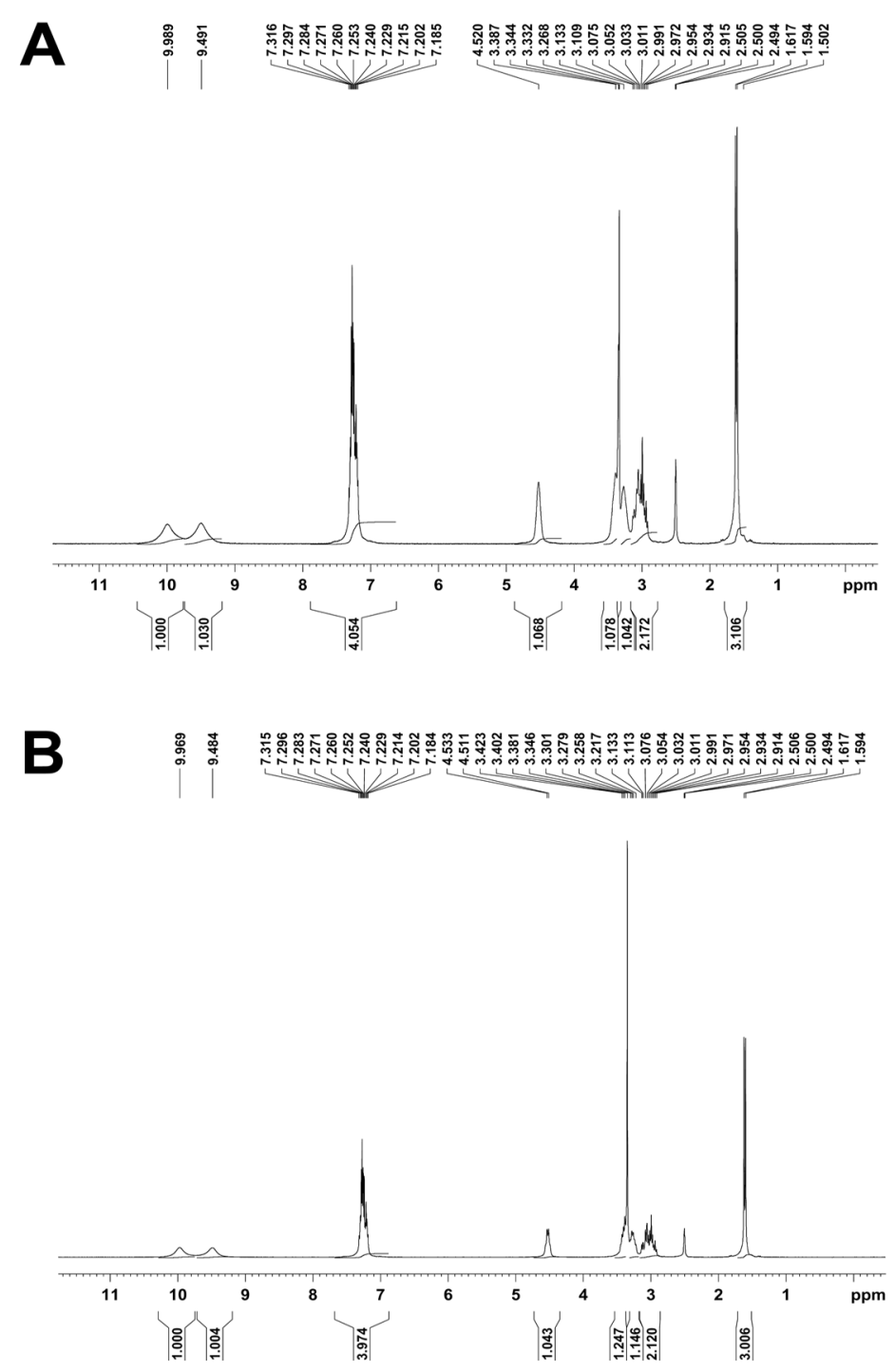

Figure A-3. ${ }^{1} \mathrm{H}$ NMR spectra of 1-methyl-TIQ.

Comparison of chemical shift (lower bracketed values) and peak integration (upper values) at baseline (A) and after 28 days of heat exposure at $60^{\circ} \mathrm{C}(\mathrm{B})$ reveals no significant structural changes after thermal challenge. 

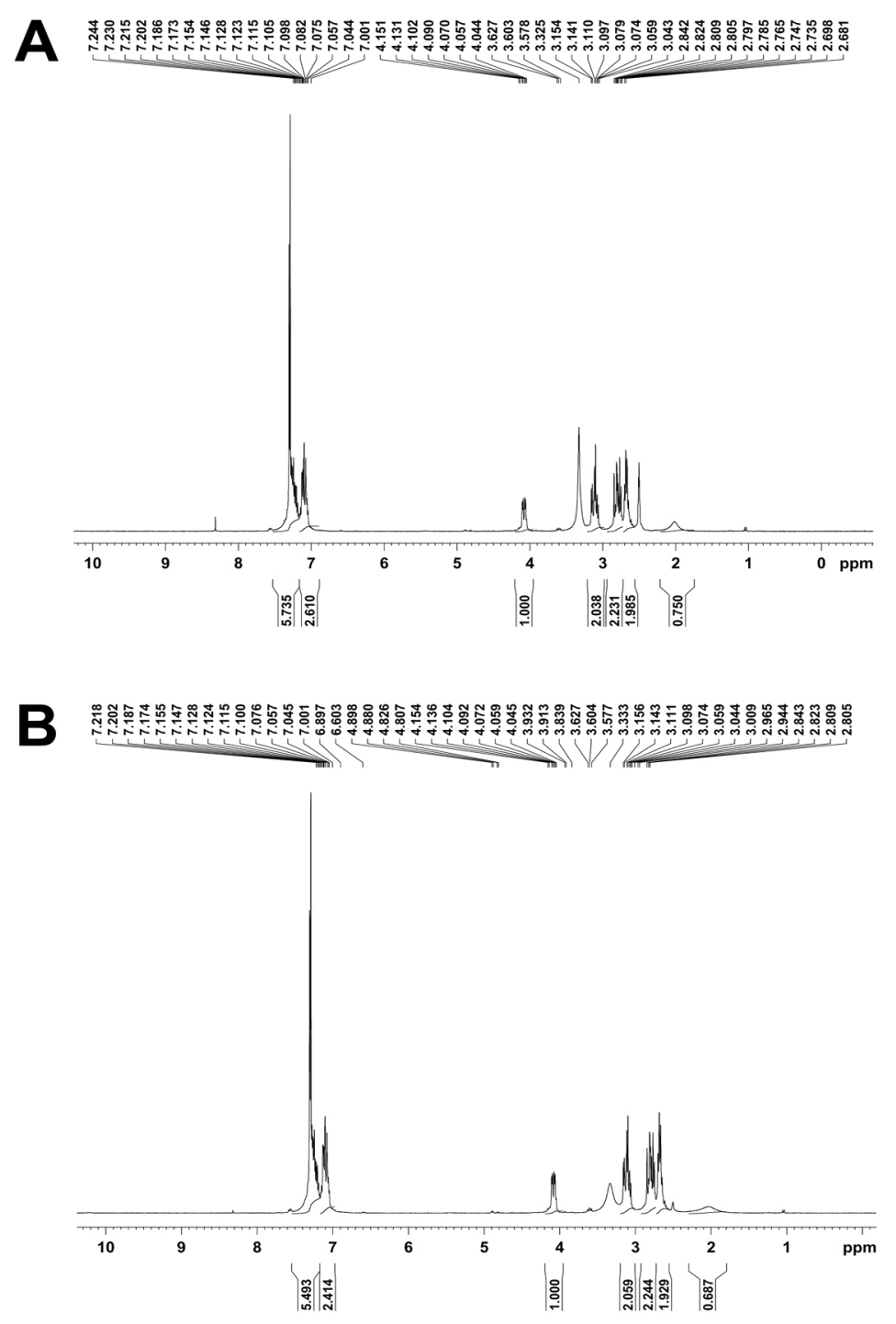

Figure A-4. ${ }^{1} \mathrm{H}$ NMR spectra of 1-benzyl-TIQ.

Comparison of chemical shift (lower bracketed values) and peak integration (upper values) at baseline (A) and after 28 days of heat exposure at $60^{\circ} \mathrm{C}(\mathrm{B})$ reveals no significant structural changes after thermal challenge. 

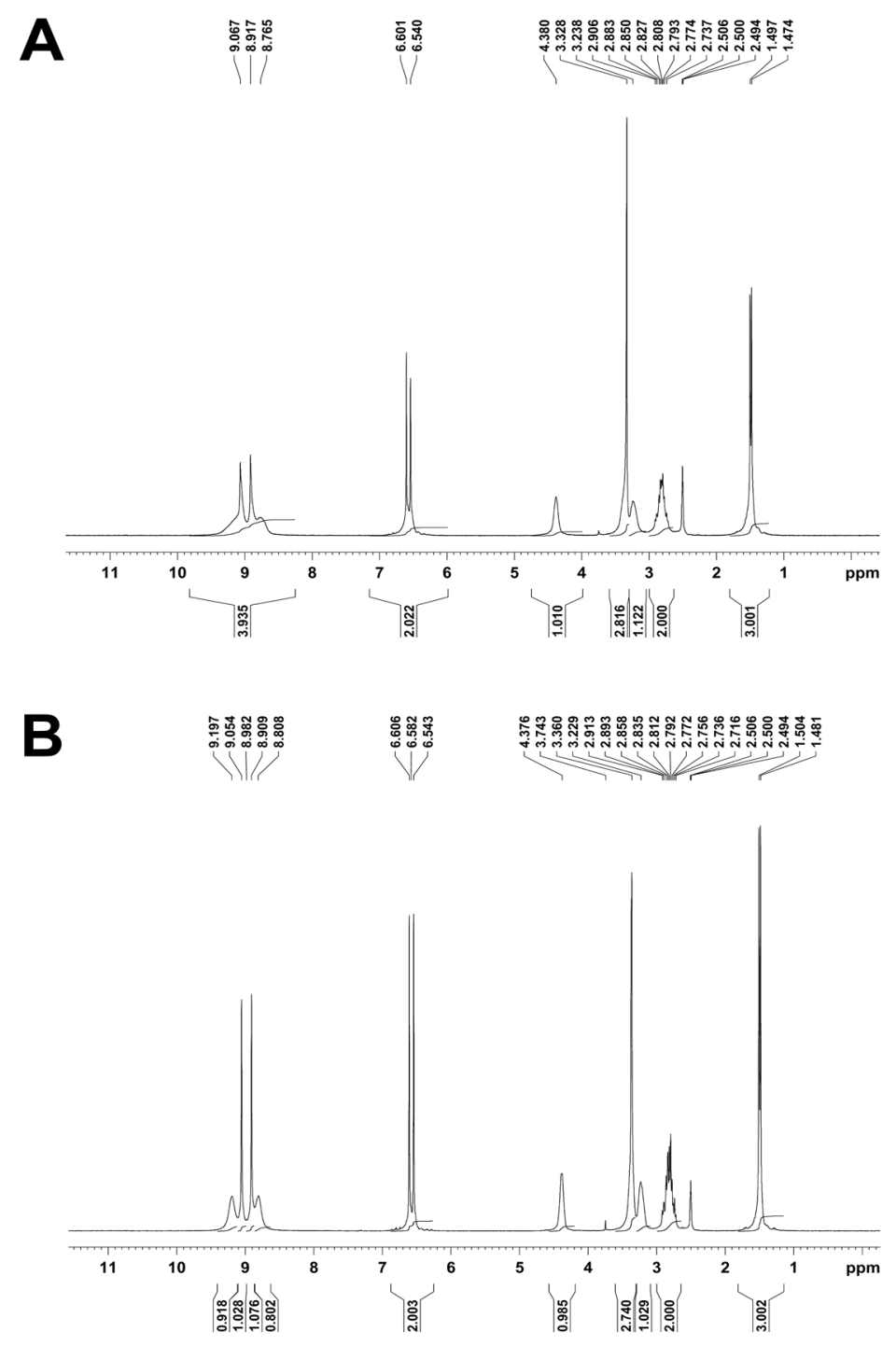

Figure A-5. ${ }^{1} \mathrm{H}$ NMR spectra of $(R / S)$ salsolinol.

Comparison of chemical shift (lower bracketed values) and peak integration (upper values) at baseline (A) and after 28 days of heat exposure at $60^{\circ} \mathrm{C}(\mathrm{B})$ reveals no significant structural changes after thermal challenge. 

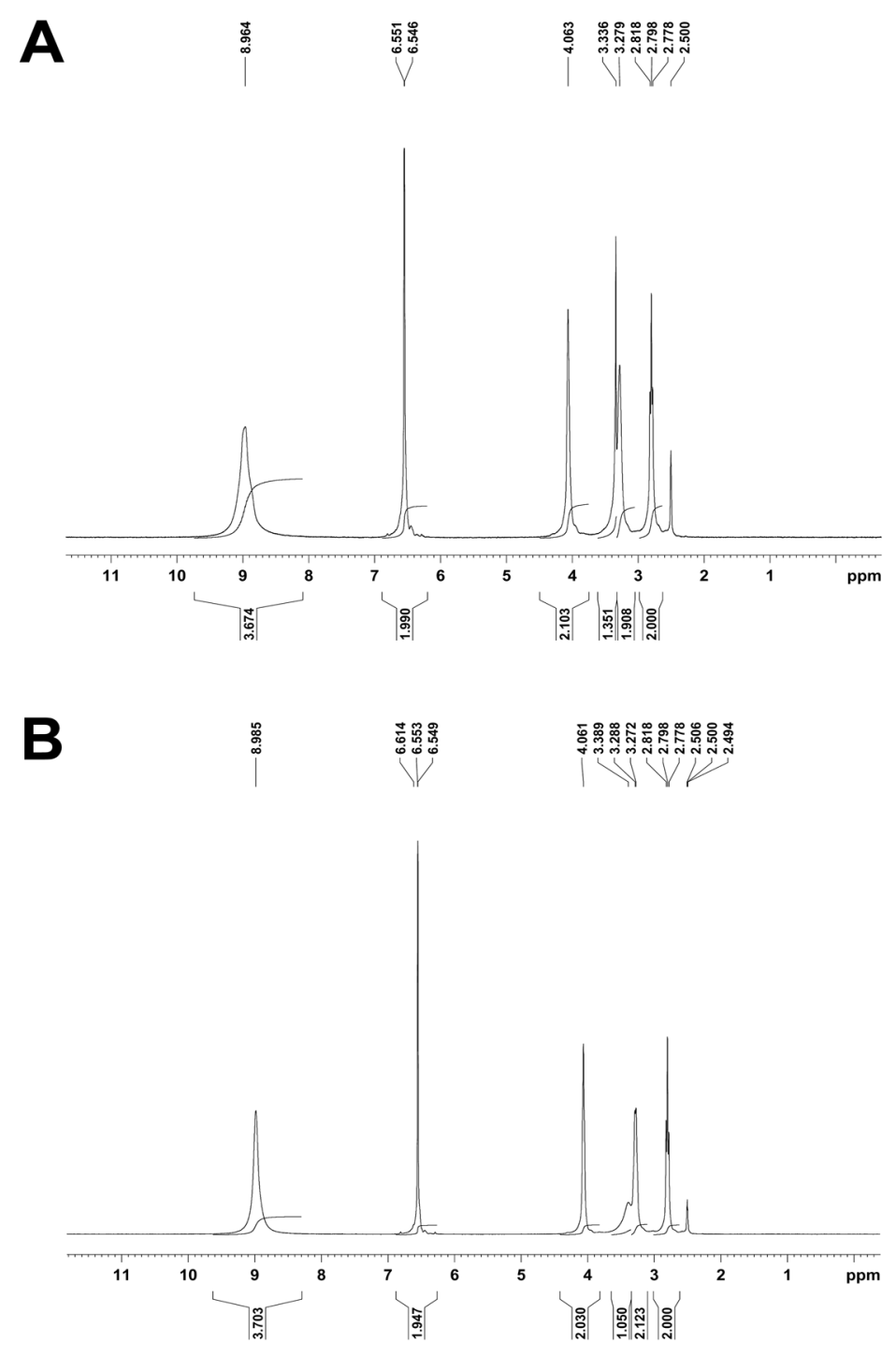

Figure A-6. ${ }^{1} \mathrm{H}$ NMR spectra of norsalsolinol.

Comparison of chemical shift (lower bracketed values) and peak integration (upper values) at baseline (A) and after 28 days of heat exposure at $60^{\circ} \mathrm{C}(\mathrm{B})$ reveals no significant structural changes after thermal challenge. 


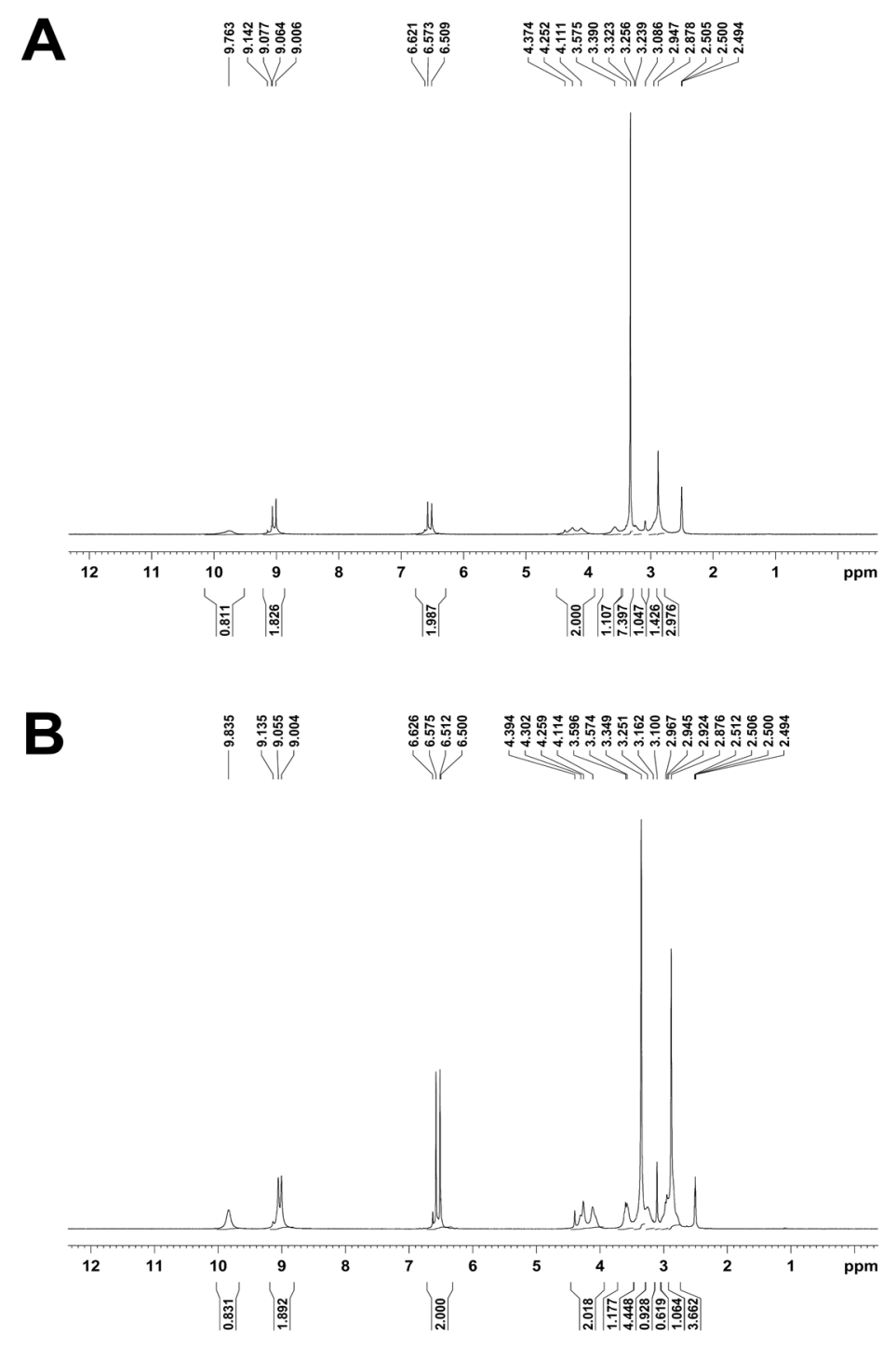

Figure A-7. ${ }^{1} \mathrm{H}$ NMR spectra of $N$-methyl-(R/S)salsolinol.

Comparison of chemical shift (lower bracketed values) and peak integration (upper values) at baseline (A) and after 28 days of heat exposure at $60^{\circ} \mathrm{C}(\mathrm{B})$ reveals no significant structural changes after thermal challenge. 

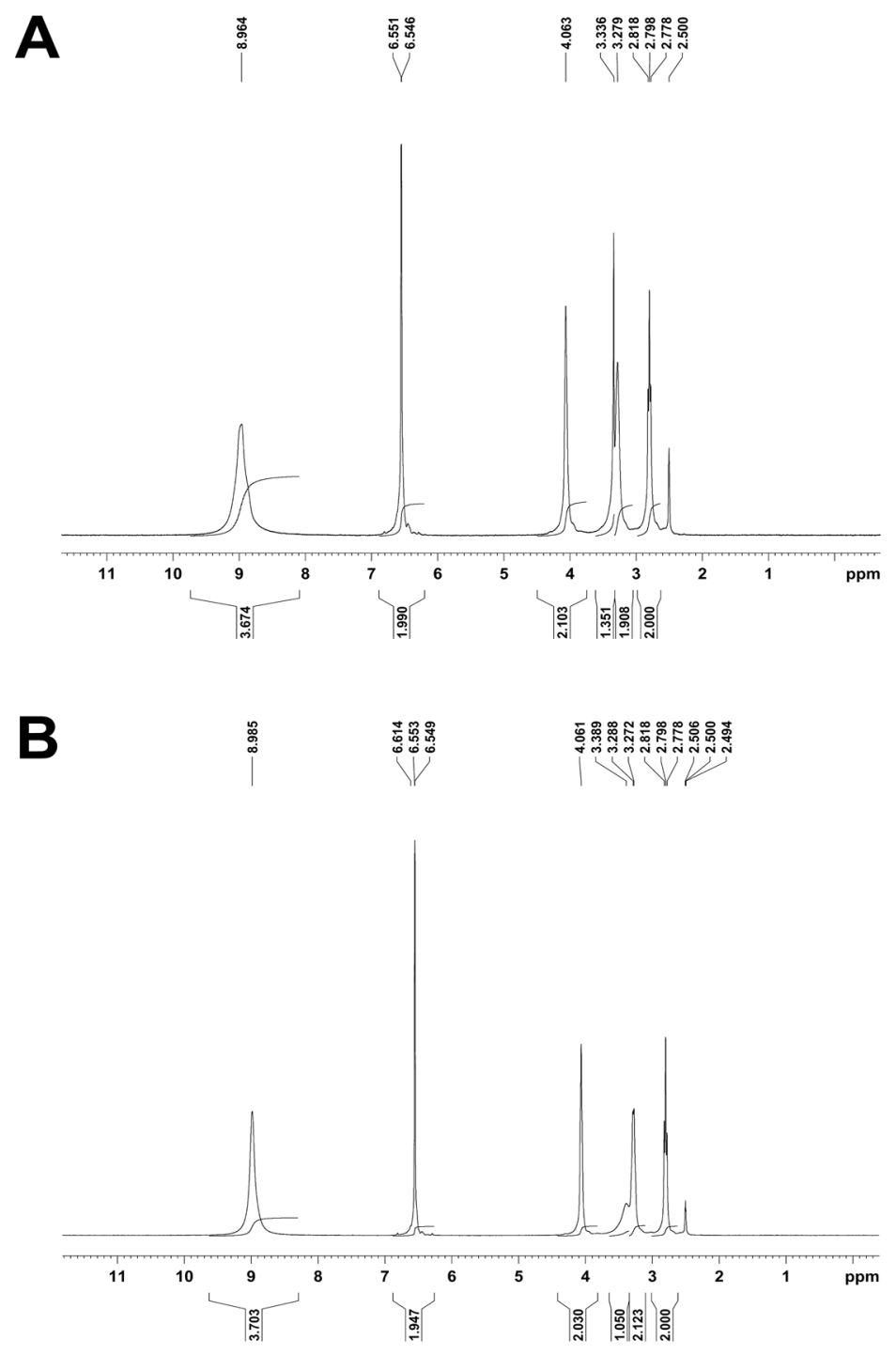

Figure A-8. ${ }^{1} \mathrm{H}$ NMR spectra of $\boldsymbol{N}$-methyl-norsalsolinol.

Comparison of chemical shift (lower bracketed values) and peak integration (upper values) at baseline (A) and after 28 days of heat exposure at $60^{\circ} \mathrm{C}(\mathrm{B})$ reveals no significant structural changes after thermal challenge. 
Table A-2. Molecular properties of TIQ derivatives and related compounds.

\begin{tabular}{lcccc}
\hline Compound & Log P & TPSA $\left(\AA^{2}\right)$ & MW $(\mathbf{g} / \mathbf{m o l})$ & $V_{\mathbf{m}}\left(\mathbf{c m}^{3} / \mathbf{m o l}\right)$ \\
\hline TIQ & 1.489 & 12.027 & 133.194 & 136.010 \\
1-Methyl-TIQ & 2.048 & 12.027 & 147.221 & 152.596 \\
N-Methyl-TIQ & 1.734 & 3.238 & 147.221 & 152.952 \\
1-Benzyl-TIQ & 3.476 & 12.027 & 223.319 & 224.246 \\
(R/S)Salsolinol & 1.056 & 52.483 & 179.219 & 168.632 \\
N-Methyl-(R/S)Salsolinol & 1.301 & 43.694 & 193.246 & 185.575 \\
Norsalsolinol & 0.496 & 52.483 & 165.192 & 152.045 \\
N-Methyl-Norsalsolinol & 0.741 & 43.694 & 179.219 & 168.988 \\
Rotenone & 3.585 & 63.241 & 394.423 & 348.335 \\
MPTP & 2.330 & 3.238 & 173.259 & 180.369 \\
MPP+ & -2.726 & 3.885 & 170.235 & 171.089 \\
Reticuline & 2.375 & 62.162 & 329.396 & 308.315 \\
\hline
\end{tabular}

Log $P=$ Octanol/Water Partition Coefficient. TPSA $=$ Topological Polar Surface Area.

$\mathrm{MW}=$ Molecular Weight.

$V_{\mathrm{m}}=$ Molecular Volume. 
APPENDIX B. PARKINSON DISEASE LIFETIME FRUIT AND VEGETABLE INTAKE QUESTIONNAIRE 
Dear Research Subject,

Thank you for taking the time to fill out this survey, which is part of a research study. With growing evidence that the development of Parkinson disease (PD) may be influenced by environmental factors, we are interested in the role that dietary habits may play in the progression of this disease. This survey will present a number of questions about your lifetime intake of various foods and should take about 30 minutes to complete. Although you will not be asked any sensitive questions, you may become tired while completing the survey. If so, please feel free to take a break and return the survey in the envelope provided. This survey is voluntary and if you do not wish to participate your medical care will not be affected in any way. If you have any questions or concerns at any time, please feel free to contact your questionnaire administrator. You willingness to complete this questionnaire will indicate that you freely consent to participate. It is with high hopes that information gained from this study can be used in the future for the prevention and/or treatment of PD. Again, thank you for taking the time to participate in this study.

Sincerely, Mark S. LeDoux, M.D., Ph.D. and Ronald F. Pfeiffer, M.D. University of Tennessee Health Science Center Department of Neurology 855 Monroe Avenue, Link Building Suite 415

Memphis, Tennessee 38163

Phone: 901-448-1662

\section{General Instructions:}

Please estimate, on average, how many servings of the following foods you ate every month during the indicated age ranges of your life. Please do not skip any foods.

Please use the following symbols when answering questions:

\begin{tabular}{|c|c|c|c|}
\hline Never & $\begin{array}{c}\text { A few times per year } \\
\text { (< once per month) }\end{array}$ & $\begin{array}{c}\text { One serving per } \\
\text { month }\end{array}$ & $\begin{array}{c}\text { If > one serving per month, write } \\
\text { the number }\end{array}$ \\
\hline$\varnothing$ & + & 1 & $2,3,4$, etc. \\
\hline
\end{tabular}

For example, if you never ate a food, write " $\varnothing$ " in the box. If you ate a serving of food greater than $(>)$ once per month, write the number of servings you ate of that food per month. If you only ate a serving of food a few times per year or less than $(<)$ one serving per month, write "+" in the box. Your study administrator will show you pictures and cup sizes to help you estimate serving sizes.

Age (Years):

Gender (Male or Female):

Weight (lbs.):

Race:

Height (ft.):

Survey Date:

\section{Please place a check mark next to ONE of the following:}

I have been diagnosed with Parkinson disease

I have NOT been diagnosed with Parkinson disease (control subject) 
Part 1. Using the symbols in the answer key above, write the number of servings of fresh fruits you consumed every month in the box under the indicated age ranges. One serving equals approximately one medium sized fresh fruit, one half cup of cut fruit, or six ounces of fruit juice.

\begin{tabular}{|l|l|l|l|l|}
\hline Fruit & $\mathbf{0 - 2 0}$ Years & $\mathbf{2 1 - 4 0}$ Years & $\mathbf{4 1 - 6 0}$ Years & $>60$ Years \\
\hline Banana & & & & \\
\hline Apple & & & & \\
\hline Watermelon & & & & \\
\hline Orange & & & & \\
\hline Cantaloupe & & & & \\
\hline Grape & & & & \\
\hline Grapefruit & & & & \\
\hline Strawberry & & & & \\
\hline Peach & & & & \\
\hline Pear & & & & \\
\hline Nectarine & & & & \\
\hline $\begin{array}{l}\text { Honeydew } \\
\text { (melon) }\end{array}$ & & & & \\
\hline Plum & & & & \\
\hline Avocado & & & & \\
\hline Lemon & & & & \\
\hline Pineapple & & & & \\
\hline Tangerine & & & & \\
\hline Cherry & & & & \\
\hline Kiwi fruit & & & & \\
\hline Lime & & & \\
\hline
\end{tabular}


Part 2. Using the symbols in the answer key above, write the number of servings of fresh or cooked vegetables you consumed every month in the box under the indicated age ranges. One serving equals approximately one cup leafy vegetables or one half cup of other vegetables.

\begin{tabular}{|l|l|l|l|l|}
\hline Vegetable & $\mathbf{0 - 2 0}$ Years & $\mathbf{2 1 - 4 0}$ Years & $\mathbf{4 1 - 6 0}$ Years & $>60$ Years \\
\hline Potato & & & & \\
\hline $\begin{array}{l}\text { Lettuce } \\
\text { (iceberg) }\end{array}$ & & & & \\
\hline Tomato & & & & \\
\hline Onion (yellow) & & & & \\
\hline Carrot & & & & \\
\hline Celery & & & & \\
\hline Corn & & & & \\
\hline Broccoli & & & & \\
\hline Cabbage & & & & \\
\hline Cucumber & & & & \\
\hline Bell Pepper & & & & \\
\hline Cauliflower & & & & \\
\hline Lettuce (leaf) & & & & \\
\hline Sweet potato & & & & \\
\hline Mushroom & & & & \\
\hline Green onion & & & & \\
\hline Green bean & & & & \\
\hline Radish & & & & \\
\hline Squash & & & & \\
\hline Asparagus & & & \\
\hline
\end{tabular}




\section{VITA}

Michael G. DeCuypere was born in Hampton, Virginia on October 1, 1978. After graduating from Phoebus High School in 1996, he attended the Tennessee

Technological Unieversity in Cookeville, Tennessee, where he graduated with highest honors with a bachelor's degree in biochemistry. After graduation, he began study in the combined degree MD/PhD program at the University of Tennessee Health Science Center in Memphis, Tennessee. In August 2004, he began his doctoral education in the Neuroscience Program. His doctoral dissertation research was carried out in Dr. Mark S. LeDoux's laboratory in the Department of Neurology. In May 2010, he successfully completed the requirements for both his Doctor of Medicine and Doctor of Philosophy degrees. In July of 2010, he will begin residency training in the University of Tennessee Department of Neurosurgery. 